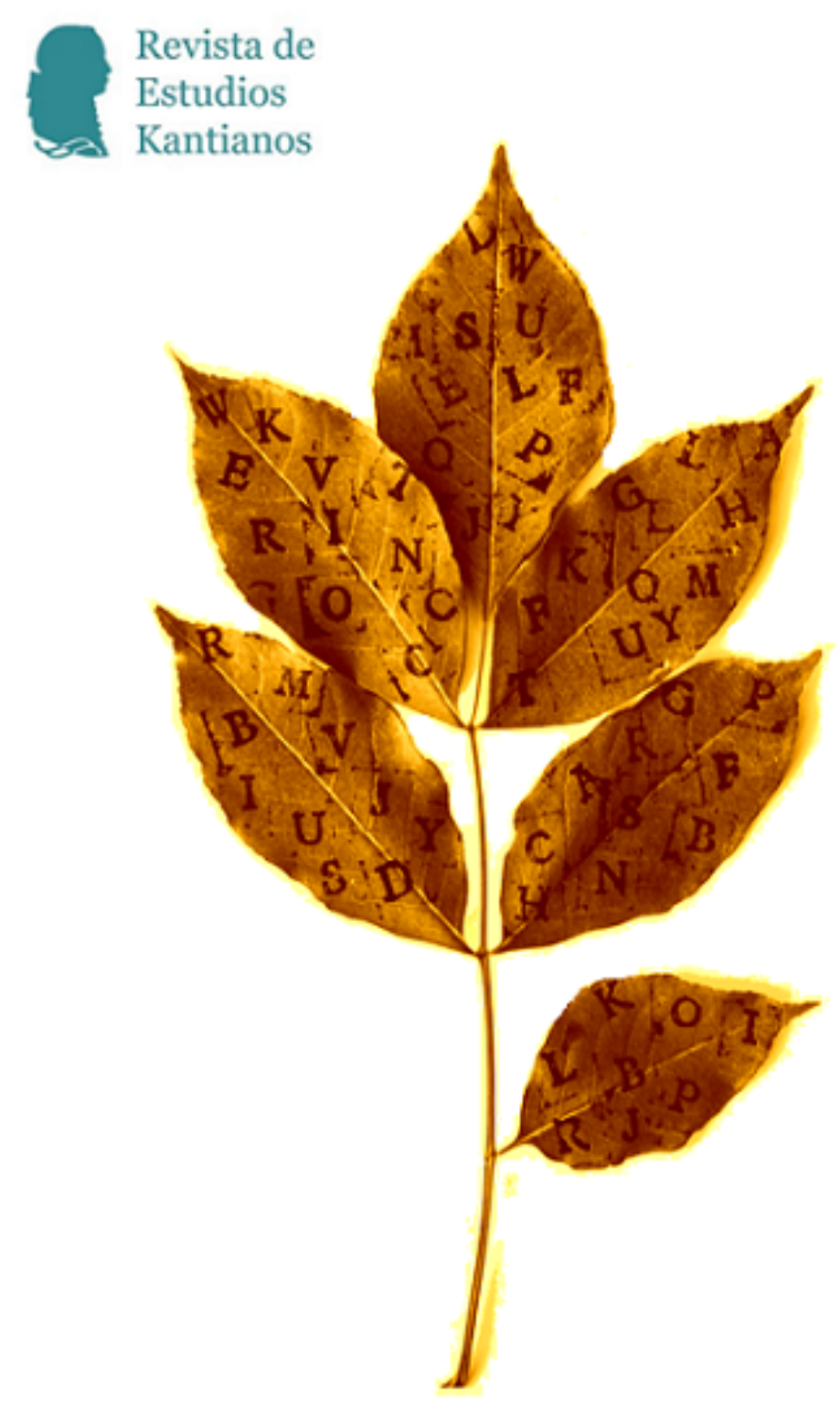


Revista de Estudios Kantianos 


\section{Revista de Estudios Kantianos}

Publicación internacional de la Sociedad de Estudios Kantianos en Lengua Española Internationale Zeitschrift der Gesellschaft für Kant-Studien in Spanischer Sprache

International Journal of the Society of Kantian Studies in the Spanish Language

\section{Dirección}

Pedro Jesús Teruel, Universitat de València

pedro.teruel@uv.es

Hernán Pringe, CONICET-Universidad de Buenos Aires/

Universidad Diego Portales, Santiago de Chile

hpringe@gmail.com

\section{Secretario de edición}

Fernando Moledo, Universidad de Buenos Aires - CONICET

fernandomoledo@filo.uba.ar

\section{Secretaria de calidad}

Marcela García, Universidad de Morelia, México

garciar.marcela@gmail.com

\section{Editores científicos}

Jacinto Rivera de Rosales, UNED, Madrid

Claudia Jáuregui, Universidad de Buenos Aires

Vicente Durán, Pontificia Universidad Javeriana, Bogotá

Julio del Valle, Pontificia Universidad Católica del Perú, Lima

Jesús Conill, Universitat de València

Gustavo Leyva, Universidad Autónoma de México, México D. F.

María Xesús Vázquez Lobeiras, Universidade de Santiago de Compostela

Wilson Herrera, Universidad del Rosario, Bogotá

Pablo Oyarzun, Universidad de Chile, Santiago de Chile

Paula Órdenes Azúa, Universität Heidelberg 


\section{Comité científico}

Juan Arana, Universidad de Sevilla

Reinhardt Brandt, Philipps-Universität Marburg

Mario Caimi, Universidad de Buenos Aires

Monique Castillo, Université de Paris-Est

Adela Cortina, Universitat de València

Bernd Dörflinger, Universität Trier

Norbert Fischer, Universität Eichstätt-Ingolstadt

Miguel Giusti, Pontificia Universidad Católica del Perú

Dulce María Granja, Universidad Nacional Autónoma de México

Christian Hamm, Universidad Federal de Santa María, Brasil

Dietmar Heidemann, Université du Luxembourg

Otfried Höffe, Universität Tübingen

Claudio La Rocca, Università degli Studi di Genova

Juan Manuel Navarro Cordón, Universidad Complutense, Madrid

Carlos Pereda, Universidad Nacional Autónoma de México

Gustavo Pereira, Universidad de la República, Uruguay

Ubirajara Rancan de Azevedo, Universidade Estadual Paulista, Brasil

Margit Ruffing, Johannes Gutenberg-Universität Mainz

Gustavo Sarmiento, Universidad Simón Bolívar, Venezuela

Sergio Sevilla, Universitat de València

Roberto Torretti, Universidad Diego Portales, Santiago de Chile

Violetta Waibel, Universität Wien

Howard Williams, University of Aberystwyth

Allen W. Wood, Indiana University

Diseño, revisión de estilo y maqueta

Josefa Ros Velasco, Harvard University, Cambridge (MA)

\section{Corrector}

Aldo Perán, Universidad Diego Portales, Santiago de Chile

\section{Entidades colaboradoras}

Sociedad de Estudios Kantianos en Lengua Española (SEKLE)

Departament de Filosofia de la Universitat de València

Instituto de Humanidades, Universidad Diego Portales

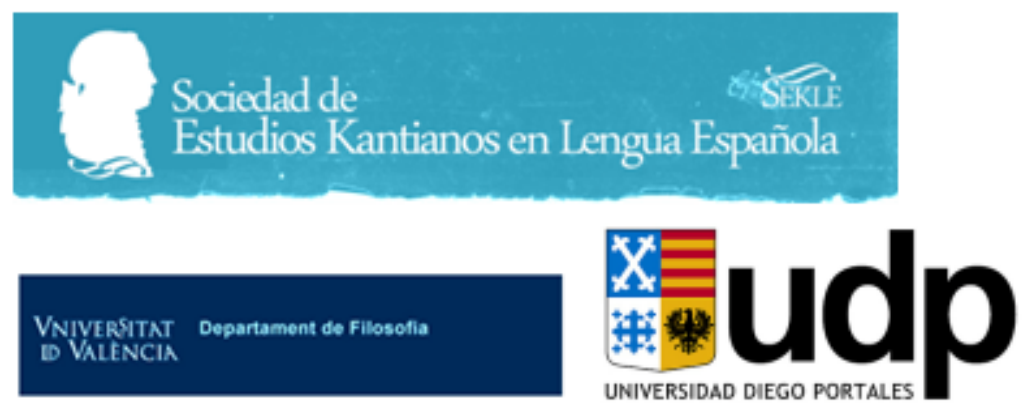




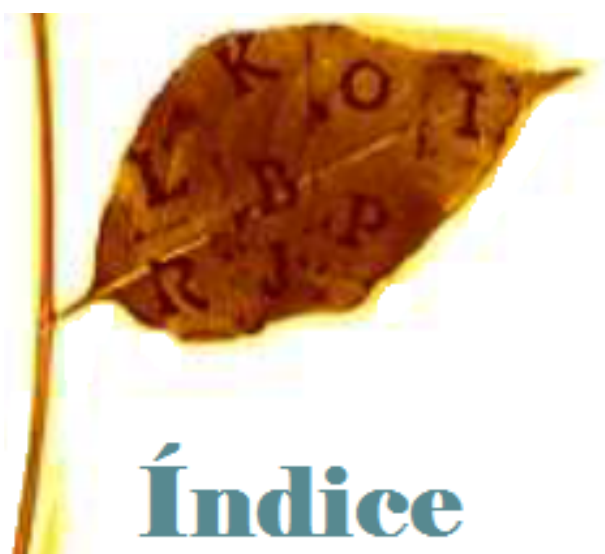

Artículos

125 Los deberes del amor en la doctrina kantiana de la virtud

Bernd Dörflinger

DOI 10.7203/REK.2.2.10807

135 Towards a new conception of metaphysics: Lambert's criticism on Wolff's mathematical method

Gesa Wellmann

DOI 10.7203/REK.2.2.10724

149 Acerca del alcance objetivo de las prescripciones metodológicas de la función regulativa de la razón teórica en la Crítica de la razón pura de Kant

Martín Arias Albisu

DOI 10.7203/REK.2.2.8432

168 Formas de autoconciencia en la "Reflexión de Leningrado"

Matías Hernán Oroño

DOI 10.7203/REK.2.2.8799

179 Kants Konzeption kosmologischer Freiheit - ein metaphysischer Rest?

Christian Krijnen

DOI 10.7203/REK.2.2.10407

\section{Informe}

196 VII Coloquio Multilateral-Kant 2017

Paula ÓrdenesAzúa

DOI 10.7203/REK.2.2.10756 


\section{Reseñas}

200 Alfredo Ferrarin: Il pensare e l'io. Hegel e la critica di Kant. Roma, Carocci Editore, 2016, 244 pp. ISBN: 978-88-430-8246-9.

Luigi Filieri

DOI 10.7203/REK.2.2.10764

205 Mario Caimi, Ileana Beade, José González Ríos, Macarena Marey, Fernando Moledo, Mariela Paolucci, Hernán Pringe y Marcos Thisted: Diccionario de la filosofía crítica kantiana. Buenos Aires, Colihue, 2017, 507 pp. ISBN: 978-950-563-450-7.

Miguel Herszenbaun

DOI 10.7203/REK.2.2.10499

210 Paula Órdenes Azúa y Daniela Alegría: Kant y el Criticismo: pasado, presente, y ifuturo?, Porto Alegre, Editora Fi, 2015, 303 pp. ISBN: 978-85-66923-52-0.

Constanza Terra

DOI 10.7203/REK.2.2.10777

212 Juan Ormeño Karzulovic y Miguel Vatter (eds.): Forzados a ser libres. Kant y la teoría republicana del derecho. Santiago, Fondo de Cultura Económica, 2017, 243 pp. ISBN: 978956-289-149-3.

Cristóbal Olivares

DOI 10.7203/REK.2.2.10762

215 Immanuel Kant (ed. y trad. Gonzalo Serrano): La Deducción trascendental y sus inéditos, 1772-1788. Bogotá, Universidad Nacional de Colombia, 2014, 321 pp. ISBN: 978-95-876195-39.

Pedro Stepanenko

DOI 10.7203/REK.2.2.10763

\section{Semblanza}

220 Juan Adolfo Bonaccini (16 de mayo de 1965 / 10 de julio de 2016)

Daniel Omar Perez

DOI 10.7203/REK.2.2.10783

\section{Convocatorias y normas para autores}

223 Kant en México

DOI 10.7203/REK.2.2.10863

224 IV Congreso Internacional de la SEKLE, Valencia, 2018

DOI 10.7203/REK.2.2.10863

227 VIII Coloquio Multilateral-Kant 2018

DOI 10.7203/REK.2.2.10863

229 Normas para autores

DOI 10.7203/REK.2.2.10863 


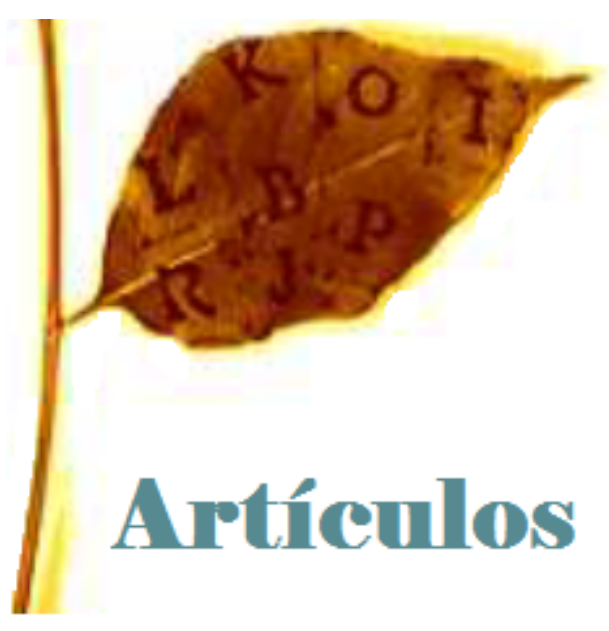




\title{
Los deberes del amor en la doctrina kantiana de la virtud. Su ubicación en el límite entre razón y sentimiento
}

\author{
BERND DÖRFLINGER ${ }^{1}$
}

\begin{abstract}
Resumen
La presente contribución ha de ser considerada un tentativo de entender la noción de amor contenida en el concepto 'deber del amor' -no detalladamente desplegado por Kant y, por ello, altamente necesitado de explicación-, y de entenderla en tanto que tiene siempre una significación emotiva y se halla siempre sintéticamente enlazada con el concepto del deber -que siempre tiene una significación intelectual-, de manera que se trata siempre de una correlación libre de contradicciones. Por decirlo en el lenguaje de las facultades, dicha correlación descansa en el nexo ininterrumpido entre la facultad de desear -aquí la superior, la intelectual, que instituye un deber- y la facultad del placer y del displacer, que muestra una reacción emotiva.
\end{abstract}

Palabras clave: amor, placer, complacencia, benevolencia, interés

\section{Die Liebespflichten in Kants Tugendlehre Ihre Verortung im Grenzgebiet von Vernunft und Gefühl}

\begin{abstract}
Der vorliegende Beitrag ist als Versuch zu betrachten, den im Begriff der Liebespflicht enthaltenen Liebesbegriff, der von Kant nicht detailliert entfaltet und also höchst erklärungsbedürftig ist, so zu verstehen, dass er immer eine Gefühlsbedeutung hat und dass er mit dem Pflichtbegriff, der immer eine intellektuelle Bedeutung hat, synthetisch verbunden ist, dass es sich also um eine widerspruchsfreie Korrelation handelt. Diese beruht auf dem nie suspendierten Verhältnis zwischen dem Begehrungsvermögen, hier dem oberen, dem intellektuellen, das eine Pflicht statuiert, und dem Vermögen der Lust und Unlust, das eine Gefühlsreaktion zeigt.
\end{abstract}

Key words: Liebe, Pflicht, Wohlgefallen, Wohlwollen, Interesse

En el pasaje en el que Kant, en el marco de la Metafisica de las costumbres, introduce los deberes de virtud respecto de otros seres humanos, señala como objeto de dichos deberes -como la finalidad que sería a la vez deber- la felicidad ajena (cf. MS, AA 06: 393.11). De las dos especificaciones expuestas aquí -el bienestar físico de los demás y su integridad moral-, la primera requiere un hacer y la segunda un abstenerse. Se requiere abstenerse de llevar a los demás a la tentación de una falta moral, para que su felicidad no venga disminuida por remordimientos de conciencia; se requiere hacer lo preciso para servir a sus legítimas exigencias, para apropiárselas.

Kant considera que el cumplimiento de estos deberes es difícil, "sobre todo, cuando ha de suceder no por inclinación" -entre paréntesis pone "amor"- "hacia los otros, sino por deber, con sacrificio y humillación de alguna concupiscencia" (MS, AA 06: 393.14-16). El aquí denominado 'amor' no es, pues, condición explícita para la validez y la realización de los deberes. Ahora bien, no es sólo que el placer asociado a dicho amor de inclinación resulte irrelevante en este contexto, sino

\footnotetext{
${ }^{1}$ Universität Trier.
} 
que se añade incluso el desagrado cuando la realización del deber requiere sacrificar el propio deseo sensual. Kant determina ulteriormente el deber de beneficencia como sigue: "[...] he de sacrificar una parte de mi bienestar a los demás, sin esperanza de una contrapartida" (MS, AA 06: 393.24 s.). Con la expulsión de la esperanza en la contrapartida, del proceso del cumplimiento del deber se excluye también la 'expectativa' del placer.

El desagrado parece ser, pues, inevitable correlato emotivo ${ }^{2}$ del cumplimiento del deber; y esto, no sólo en el caso de los dos deberes citados, sino en el caso de todo deber: "El concepto del deber es ya en sí el concepto de un apremio (coacción) del libre arbitrio por parte de la ley", de manera que los seres humanos, "aun cuando lo sigan, lo hacen, sin embargo, de mala gana (con la resistencia de su inclinación)" (MS, AA 06: 379.15-24). En otro lugar, y de manera más compacta: "[...] Deber [...] es un apremio a una finalidad asumida de mala gana" (MS, AA 06: 386.05 s.). Ciertamente, en el caso de los imperativos de la virtud, el ser humano no es un mero receptor del precepto, sino -en otro sentido- también su emisor, y la coacción ejercitada no es externa -como en el caso de las leyes jurídicas- sino "autocoacción" (MS, AA 06: 379.17); con todo, eso no altera lo desagradablemente coactivo de la situación. Concuerda con ello que Kant califique a la virtud, empleando lenguaje bélico, de "valentía" así como de "fortitudo moralis" desplegada, en concreto, "frente al enemigo de la disposición moral en nosotros" (MS, AA 06: 380.14-16). Cotejándolo con el ser humano, se refiere a un ser imaginario, que "hiciera todo conforme a la ley" porque en él no se da "ningún impulso obstaculizador" -a saber, procedente de la sensualidad patológica- "contrario a la ley de su voluntad", como ser "santo" [sobrehumano]" (MS, AA 06: 405.13-15). El hacer-con-gusto conforme a la ley se halla en la segunda Crítica identificado con el hacer por amor y se le atribuye el "ideal de santidad"; sin embargo, resulta "inalcanzable para cualquier criatura" (KpV, AA 05: 83.24 s.). En cualquier caso, en lo que atañe al ser humano todo deber es apremio y autocoacción. A tal respecto afirma Kant, breve y concisamente: "Pero lo que se hace por coacción, no sucede por amor" (MS, AA 06: 401.34 s.).

El cuadro pergeñado hasta aquí es, en cierta medida, oscuro. Como es sabido, dio pie a Schiller a reclamar que a la realización del deber se le añadiese la cualidad estética de la gracia. La réplica de Kant es igualmente conocida. A su parecer, las "gracias" [...], cuando se trata sólo del deber", habrían de "mantenerse a respetuosa distancia" (RGV, AA 06: 23.34 s., nota).

Sin embargo, con el aspecto sentimental acentuado hasta ahora -a saber, el del desagrado asociado a una sensibilidad puesta bajo la coacción de la razón práctica- no se agota en absoluto la temática del sentimiento en la Metafisica de las costumbres. En particular, el del amor no es un asunto resuelto, como se percibe echando un vistazo al índice. La decimosegunda sección de la introducción a la doctrina de la virtud trata explícitamente del amor humano (MS, AA 06: 401-402), y lo hace en cuanto prenoción estética de la receptividad del ánimo a los conceptos del deber en general (cf. MS, AA 06: 399.02 s.). Así pues, parece que hay aún otro amor, diferente de ese amor característico de la "inclinación" sensible "a los otros" (MS, AA 06: 393.14) que Kant quiere ver fuera del proceso de realización de los deberes relativos a los demás; este otro amor habría de ser, tras esta breve caracterización, un amor que no sólo podría mantenerse sin contradicción junto a los conceptos del deber, sino que incluso promovería la efectividad de dichos conceptos.

\footnotetext{
${ }^{2}$ [Nota del traductor] "Correlato afectivo”: en el original, Gefühlskorrelat. El sustantivo alemán Gefühl ('sentimiento') aparece en el texto en posiciones adjetivas que aconsejan modular su traducción. En efecto, la versión literal de Gefühlskorrelat ("correlato sentimental') sugeriría una cualificación psíquica de aquello de lo que se habla que no se halla del todo conforme con el marco semántico; lo mismo sucede, páginas adelante, con Gefühlsresultat, Gefühlswirkung o Gefühlsmäßig. El motivo de ello es la diferencia de matices en el empleo del vocablo -Gefühl y 'sentimiento'- en ambas lenguas. Para evitar equivocidades innecesarias he optado por términos - procedente de campos semánticos próximos y más conformes al uso castellano- asociados a lo 'emotivo' o lo 'afectivo'. Así, no hablaremos de "correlato sentimental", "resultado sentimental" y "efecto sentimental" o "desde el punto de vista sentimental" sino de "correlato emotivo", "resultado emotivo" y "efecto emotivo" o "desde el punto de vista emotivo", respectivamente. En los casos en que resulta posible emplearlos sin incurrir en equivocidades, he optado igualmente por los términos del campo semántico del 'afecto': así, cuando se habla de gefühlsindifferent o de Gefühlskomponente traducimos "afectivamente indiferente" y "componente afectivo".
} 
Por lo demás, la ojeada al índice muestra que los deberes de virtud hacia otros -por lo tanto, y por ejemplo, el deber relativo al bienestar (desde el punto de vista de las inclinaciones, en absoluto carente de placer)- quedan caracterizados ellos mismos como deberes del amor. ${ }^{3}$ Toda una serie de deberes -a excepción del deber del respeto- cae pues, igualmente, bajo la denominación "deber del amor hacia otros seres humanos" (MS, AA 06: 448.07): los deberes de beneficencia, de gratitud y de empatía emocional. Todo ello requiere con urgencia una explicación y resulta incluso chocante; y es que, en el lugar en que aparecen, Kant no proporciona indicación alguna de que en la fundamentación o en el cumplimiento de los deberes hacia los demás pueda estar implicada la índole afectiva del amor humano, que en cuanto amor se habría de establecer en la dimensión positiva del espectro placerdesagrado y que resultaría bien determinante para la posterior denominación.

Los argumentos de la deducción introductoria de los deberes hacia los demás -que aquí no podemos reproducir en detalle, pero sí a grandes rasgos- son básicamente los siguientes: toda acción precisa de un fin, de un objeto propio (cf. MS, AA 06: 384-385). Las acciones que se siguen de las tendencias sensibles tienen un fin dado por la Naturaleza: la propia felicidad; prescribirlo sería, pues, absurdo. La felicidad ajena no está contenida en el fin natural dado; y es que según el fin natural cada uno es su prójimo. Así pues, dicho fin natural puede ser, obviamente, "contrario al deber" (MS, AA 06: 380.26). La ética, como doctrina de los deberes de virtud por razón pura práctica, requiere un "fin contrapuesto" que no es ya uno dado de hecho sino objeto del deber; precisa además de uno que no haya de ser considerado "siempre y sólo como medio para otros fines", puesto que en este caso "un imperativo categórico" sería "imposible" (MS, AA 06: 385.16s.). Finalmente, de entre los candidatos sopesados -junto a la perfección moral de los demás, de la cual han de ocuparse ellos mismos, aun cuando no sean inducidos a la tentación- es la felicidad ajena, y sólo ésta, en cuanto fin que es a la vez deber, la que se trae a colación. La felicidad ajena es el fin apriórico que se buscaba, independiente de la inclinación. Ser independiente de la inclinación significa aquí, de nuevo, que en este escenario el otro no puede ser objeto del amor característico de la inclinación. Que aquél pueda ser destinatario de otro tipo de amor, o que un tal fuese elemento incluso necesario en la concepción de los deberes hacia los demás, no emerge en la argumentación esbozada. Al contrario: en lo concerniente a la deducción del citado deber -a su fundamentación, pues-, parece que un amor caracterizado al uso no juega papel alguno y que no se da una explicación a la denominación posterior 'deber del amor'.

He ahí la que habrá de ser en lo que sigue la principal pregunta: cómo se explica que el deber de promover la felicidad ajena haya de ser, a fin y al cabo, un deber del amor. Por parte del actor moral parece entrar, de ese modo, en escena un elementoespecífico asociado al sentimiento -cosa que habría de ser explicada ulteriormente-, nada menos que un sentimiento a priori; y es que, según Kant, la metafísica de las costumbres habrá de ser "independiente de toda condición de la intuición" (MS, AA 06: 375.04 s.). No podría darse tampoco una contradicción con la constatación kantiana de que "ningún principio moral" se funda, "como algunos suponen erróneamente, sobre un cualquier sentimiento" (MS, AA 06: 374.23 s.). La explicación buscada habría de tematizar también al destinatario del actor moral bajo el punto de vista de lo que lo hace adecuado como objeto de amor: debería mostrarse como digno de amor desde algún otro enfoque, diferente del asociado al amor sensible.

\footnotetext{
${ }^{3}$ [Nota del traductor] "Deberes de amor": en el original, Liebespflichten. La expresión nominal alemana Liebespflicht presenta una ambivalencia que en castellano puede dar lugar a tres versiones. Por un lado, con ella se alude al deber llevado a cabo por amor y no en virtud de otros motivos o móviles (como, por ejemplo, el interés propio o la coacción externa); por otro lado, con ella nos podemos referir al deber que lleva aparejado el amor, a las consecuencias prácticas asociadas a la disposición amorosa; finalmente, puede tratarse del deber cuyo contenido es el amor mismo. En el primer caso traduciremos Liebespflicht como "deber por amor"; en el segundo, como "deber del amor". El tercer concepto se presenta en el texto sólo en cuanto perífrasis verbal que puede ser resuelta, sencillamente, como "deber de amar". En el título hemos optado por la expresión "los deberes del amor", desde el punto de vista formal, por su mayor proximidad al uso filosófico y literario en general; desde el punto de vista material, porque la argumentación irá perfilando su uso hasta hacer preferible dicha interpretación (dado que, como veremos, el amor no es fuente ni contenido de dichos deberes sino afecto concomitante).
} 
Antes de afrontar esta pregunta emprenderemos, no obstante, un pequeño excursus en la sección, ya citada, en la que se trata el amor humano como prenoción estética de la receptividad hacia los conceptos del deber. Si bien -dicho sea de entrada- el amor allí tratado no será el que buscamos, parece adecuado tomarlo en consideración, con todo, para que la modulación que buscamos se recorte sobre ese horizonte. Nuestra aproximación podrá ser relativamente breve, ya que Dieter Schönecker ha dicho ya mucho, y sustancial, en torno a esta sección.

Con la acreditada acribia que caracteriza sus comentarios en el marco de su investigación kantiana, y a la vista del críptico y desconcertante texto de la debatida sección, Schönecker ha identificado, en primer lugar, de qué concepto de amor se trata -de entre los distintos conceptos de amor tomados en consideración-en el caso de la prenoción estética de la receptividad hacia los conceptos del deber. Se trata del concepto de "amor de complacencia" [amor complacentiae] (MS, AA 06: 402.22). A la pregunta inevitable -pero, en ese lugar, sin respuesta- sobre cuál pueda ser, pues, el objeto de dicho amor de complacencia, responde Schönecker: "Con 'amor de complacencia' alude Kant a la complacencia en la perfección. [...] A su vez, por 'perfección moral' entiende Kant la aspiración a la integridad y la solidez morales" (Schönecker 2010: 175). El pasaje clave que apoya esa tesis en el texto kantiano es el fragmento del parágrafo 25 -considerablemente alejado de la sección introductoria- en el que habla de un sentimiento de amor que ha de ser denominado "estético" e incluso del "amor de complacencia" en cuanto "gozo en la perfección de otros seres humanos" (MS, AA 06: 449.17-19).

Más información sobre cómo haya de ser entendido ulteriormente el amor de complacencia la puede proporcionar un tipo moral, introducido por Kant, en que dicho amor no hace acto de presencia. Se trata del misántropo estético (cf. MS, AA 06: 440.25-29). También Dieter Schönecker se sirve de él en este sentido (cf. Schönecker 2010: 165 ss.). Kant trató del misántropo estético ya en la Crítica del juicio. Alude allí a dicha misantropía como una denominada así "muy impropiamente" (KU, AA 05: 276.03), puesto que se dirigiría al ser humano, en realidad, con "benevolencia", aunque habría sido apartada "de la complacencia en el hombre por una larga y triste experiencia" (KU, AA 05: 276.05-07). Dicha misantropía impropia vendría a instalarse "con la edad" en el ánimo de "muchos hombres bienpensantes" (KU, AA 05: 276.04). Estas alusiones a la biografía de un individuo y sus experiencias sugieren considerar las relaciones entre los actores morales individuales como situación paradigmática en la que el placer -asociado al amor de complacencia- o bien el desagrado - propio del potencial misántropo estético- se presentan como reacciones emotivas a la índole moral de los demás. El hincapié en lo situacional concreto de la relación entre individuos como el lugar de la afección por el amor de complacencia queda respaldado también por la caracterización kantiana de dicha forma de amor cuando afirma que ésta sería "sólo directa" y un placer "inmediatamente enlazado con la representación de la existencia de un objeto" (MS, AA 06: 402.23 s.).

Ahora bien, ¿por qué no resulta adecuado el amor de complacencia para explicar el amor en el concepto de 'deber de amor', es decir, en la "fórmula" reconocida por Kant -pero aún por dilucidar- en la forma del imperativo "Ama a tu prójimo (al otro ser humano) como a ti mismo" (MS, AA 06: $451.28 \mathrm{~s}$.)? He aquí la respuesta: porque en el amor de complacencia se trata del resultado emotivo de una afección del sentido interno; dicho de otra manera: porque, aun no siendo amor patológico por inclinación, descansa, como éste, sobre la receptividad, asociada a una facultad particular que Kant denomina "disposición natural del ánimo" en orden a "ser afectado por los conceptos del deber" (MS, AA 06: 399.11 s.). El placer propio del amor de complacencia se presenta -o no- en cada caso dependiendo de lo que ocurre en situaciones morales relevantes en el trato de una persona con otras, en cuanto índole moral suya; lo mismo sucede con el desagrado, que, en el caso de muchas experiencias negativas similares -tal y como hemos visto- puede dar lugar al misántropo estético. 
Sobre la base de ese inapropiado carácter receptivo del amor, propio de la complacencia moral, Kant rechaza repetidamente que se pueda dar una obligación respecto de él. "El a mor es un asunto del sentimiento, no de la voluntad, y no puedo amar porque quiero, y menos aún porque debo (ser apremiado a amar); de ahí que un deber de amar sea un absurdo" (MS, AA 06: 401.24-26).

Ahora bien, esta frase resuelve un problema a la vez que suscita otro. Responde unívocamente a la pregunta de si el amor de complacencia puede ser el amor pensado en el concepto de 'deber de amor', y lo hace de forma negativa. Sin embargo, y a diferencia de lo que podría parecer en un primer momento, no resulta válida con estricta necesidad respecto de cualquier forma de amor. Si lo dicho hubiera de valer de forma estrictamente necesaria -a saber, que el amor no fuese un asunto de la voluntad, que nadie se pudiese instalar voluntariamente en el estado propio del amor y que todo deber de amar resultase un absurdo-, entonces emergería una contradicción con la "fórmula" ya citada, y en cierto modo sostenida por Kant, "ama a tu prójimo (al otro ser humano) como a ti mismo" (MS, AA 06: 451.28 s.). E igualmente vendría a resultar prácticamente un callejón sin salida la pretensión de lograr entender todavía el concepto 'deber de amor'.

De que la frase está acuñada para el amor de complacencia hay un indicio en el contexto más próximo, que, sin embargo, viene a ser relativizado en lo que sigue: "En cambio, el amor de benevolencia [amor benevolentiae] puede, en cuanto acción, venir sujeto a una ley por deber. A menudo -aunque impropiamente- se denomina también amor a una benevolencia desinteresada hacia los seres humanos" (MS, AA 06: 401.27-29). Al alivio producido por la perspectiva de que a raíz de estenuevo concepto de amor-amor benevolentiae- la prosecución de la pregunta por el sentido de la expresión 'deber de amor' parezca de nuevo prometedora, le sigue, con esa declaración, la indignación suscitada por la afirmación de que se trata de amor sólo impropiamente. Ahora bien: si el vocablo 'amor' -en la expresión 'amor de benevolencia'- no ha de resultar del todo engañoso, entonces debería tratarse en este amor de algo así como de un análogo del amor en sentido propio, sea éste lo que fuere. Junto a rasgos no coincidentes, en los que residiría lo impropio, debería haber también rasgos coincidentes, sin los cuales no vendría a darse analogía alguna. Por desgracia, Kant no llevó a cabo la comparación que en este pasaje hubiera sido precisa.

La ahora oportuna consideración ulterior del amor de benevolencia proporcionará quizá alguna clave. Entre las preguntas que han de ser respondidas se hallan, por ejemplo, las siguientes: ¿Qué constitución de ánimo o bien de disposiciones habrá de ser atribuida al actor moral que actúe conforme al amor de benevolencia? ¿Pertenece a dicha constitución un componente afectivo procedente, en concreto, del ámbito del placer propio del espectro placer-desagrado? Tiene que ser así, ya que sin esta especificidad del concepto de amor ni siquiera se podría hablar de amor en sentido impropio. Aún nos habremos de preguntar por la conciliación con el concepto 'deber de amor', así como por el objeto de dicho amor. Que otros seres humanos hayan de ser objeto del amor de benevolencia resulta evidente; no ya, en cambio, en calidad de qué. Que puedan serlo en cuanto objeto de la inclinación sensible o como objeto del amor de complacencia ha quedado ya excluido.

Respecto del amor de benevolencia se ha de subrayar ahora, en primer lugar, que con él para Kant se indica, sí, una constitución anímica, pero no sólo -e imposible que así lo fuera- una mera constitución anímica. Para ser tal, el amor de benevolencia ha de aparecer como "benevolencia práctica, efectiva", como un "hacer el bien" (MS, AA 06: 452.04 s.), motivo por el cual le asigna también la expresión sinónima "amor práctico al ser humano" (MS, AA 06: 450.31). A la benevolencia fácil, inactiva y en el mero pensamiento, la denomina Kant "benevolencia del deseo" (MS, AA 06: 452.01): desear el bien a todos -ironiza- sin contribuir a ello en nada. Eliminando el deseo reinaría entonces en el ámbito de lo fáctico el principio "cada uno en su casa y Dios en la de todos" (MS, AA 06: 452.03). 
Ahora bien, para hablar ahora del estado de conciencia que "tiene como consecuencia hacer el bien" se ha de explicar la declaración kantiana de que el amor que comparece en el marco del deber de amor se haya de identificar con la "máxima de la benevolencia" (MS, AA 06: 449.20-22). El concepto de máxima deviene así importante. Según lo establecido en el parágrafo primero de la Crítica de la razón práctica, las máximas son principios prácticos subjetivos "que contienen una determinación general del querer, bajo la cual se hallan distintas reglas prácticas" (KpV, AA 05: 19.07 s.). Las máximas no son algo que se encuentre -no se deben, pues, a la receptividad de la conciencia-, sino que ellas y su carácter universal han de ser producidas antes que nada por medio de la conciencia reflexiva autónoma. Esto aboga por la índole intelectual y en contra de la emotiva de las máximas y debería servir también para la máxima de la benevolencia por cuyo medio se expresa el amor benevolentiae. A la luz de esta explicación, el amor interpelado en el compuesto 'deber de amor' aparece como amor intelectual, no sujeto al veredicto kantiano de que no puede haber obligación alguna de tener sentimientos (cf. MS, AA 06: 449). Ahora, si el amor como sentimiento no puede ser ordenado, entonces tampoco el amor de benevolencia ni el amor del intelecto. Eso sí, si nos quedamos con este resultado, de manera que no haya sentimiento de ningún tipo que identificar en este contexto, se plantea naturalmente la pregunta de si el carácter impropio de un tal amor intelectual no sea tan grande como para hacer preferible renunciar a la expresión (asociada al sentimiento).

La índole intelectual del amor de benevolencia se confirma cuando se intenta prestar una formulación a la máxima de la benevolencia activa. Transformando el lenguaje kantiano del deber en el de las máximas, dicha formulación debería rezar como sigue: quiero "ser benevolente hacia todos los demás" (MS, AA 06: 451.05 s.). Los demás comparecen aquí en cuanto seres menesterosos; en palabras de Kant, "como prójimos", es decir, como "seres racionales necesitados", reunidos "en un lugar [...] en orden a la ayuda recíproca" (MS, AA 06: 453.13-15). En la "benevolencia en el amor universal al hombre" se trata, a su parecer, del "más grande" "según su alcance" (MS, AA 06: 451.21 s.), pues abarca a todos los seres humanos. Dicho brevemente: según eso, el objeto del amor de benevolencia es la especie 'ser humano', la Humanidad. Cuando -como debe ser-se pone por obra y la benevolencia deviene así práctica, entonces se ha de remitir, naturalmente, a un individuo; eso sí, de manera que venga a ser considerado como un caso de subsunción, a saber, como representante de la especie. En palabras de Kant: "Tomo parte en el bien de este ser humano sólo por mor del amor universal a la Humanidad” (MS, AA 06: 451.23 s.).

No otra cosa se podría haber esperado, sino la especie como destinataria del deber del amor, a partir del encabezamiento del primer capítulo en torno a los "deberes hacia los demás"; y es que, al fin y al cabo, éste anuncia el tratamiento de los deberes hacia los demás "sólo como seres humanos" (MS, AA 06: 448.05). El ser humano individual, en su configuración particular, viene a primer plano sólo en un segundo capítulo (que únicamente abarca una página pero que tiene, en la estructura del texto, el mismo rango que el primero). Tematiza la cuestión aplicada, es decir, la "aplicación de principios puros del deber a casos de la experiencia" (MS, AA 06: 468.29s.); a raíz de dicha dependencia de sus eventuales reglas de aplicación respecto de la experiencia -dicho sea de paso- no puede constituir parte alguna de la Metafísica de las costumbres, puesto que las partes de ésta han de "brotar a priori de un concepto de razón" (MS, AA 06: 469.9 s.). Con la igualmente importante cuestión aplicada se pone a la vista, por decirlo ahora de manera algo diferente, la 'multiplicidad de los sujetos' 'a la vista de su situación' -algo más, pues, que sus meros rasgos de especie-. A ello se corresponde también su estatuto moral individual y biográfico, que fue caracterizado supra como objeto del amor de complacencia o bien del desagrado moral-estético. Junto al individuo moralmente puro y al corrompido aparecen también -en una enumeración kantiana un tanto desbocada, que acaba con 'etc.'- propiedades individualizadoras indiferentes desde el punto de vista moral, como el nivel de educación, el sexo o la edad. Todos esos rasgos de los individuos -tanto los moralmente relevantes como los indiferentes- deben entrar en el cálculo si se ha de obtener reglas de aplicación, las cuales no pueden ser nunca a priori. 
En la medida en que la especie 'ser humano', la Humanidad, los individuos "en cuanto meros seres humanos" vienen a ser identificados como objeto del deber en orden al amor universal de la Humanidad, la pregunta deviene inevitable: ¿qué característica específica hace digna de amor a esta especie? Planteado de otra manera: además de la benevolencia activa ordenada intelectualmente, ¿sobre qué fundamento implícito en el concepto específico de Humanidad estaría justificado aún amar a los destinatarios de dicha benevolencia, es decir, acompañarlos con una predisposición afectivamente positiva, que afirme su existencia también desde el punto de vista emotivo?

La respuesta puede ser muy sucinta: porque cada ejemplar de la especie 'ser humano' lleva en sí la razón práctica moral, en virtud de la cual, e independientemente de su naturaleza animal, se obliga a sí mismo por imperativos incondicionados. Esta propiedad normativa contrafáctica, propia de la especie, constituye el ideal de la Humanidad; no puede malograrse a causa de las faltas de hecho de los hombres individuales empíricos. En Kant se expresa como sigue:

Todas las alabanzas que atañen al ideal de la Humanidad en su perfección moral no pueden perder nada, en lo que concierne a su realidad práctica, a causa de los ejemplos de lo contrario suministrados por lo que los seres humanos son ahora o han sido o presumiblemente serán en el futuro; la antropología, que procede a partir de meros conocimientos de experiencia, no puede, pues, producir perjuicio alguno a la antroponomía, instituida por la razón incondicionadamente normativa [...] (MS, AA 06: 405.34-406.04)

Sobre el trasfondo de la distinción realizada devienen inteligibles algunas declaraciones de Kant que, de suyo, resultan chocantes. En un lugar de la introducción a la doctrina de la virtud enuncia Kant el deber -que más tarde denominará 'deber del amor'- como sigue: "Hacer el bien a otros seres humanos conforme a nuestra capacidad es deber"; la prosecución inmediata dice así: "se les ame o no" (MS, AA 06: 402.01 s.). En la exposición de la doctrina de la virtud se dice algo similar: "La máxima de la benevolencia (el amor práctico a la Humanidad) es para todos los seres humanos un deber recíproco"; también aquí se prosigue: "se les halle dignos de amor o no" (MS, AA 06: 450.31-33). Se puede disolver lo chocante de tales afirmaciones distinguiendo los aspectos expuestos: el deber del amor se da sobre la base de que los destinatarios de la benevolencia son miembros de la especie integrada por los seres dotados de razón práctico-moral, que se somete normativamente a exigencias supremas, a saber, incondicionadas. La validez de dicho deber del amor es independiente de que en su facticidad individual y empírica -bajo el punto de vista, pues, del amor receptivo de la complacencia- los seres humanos se hayan hecho acreedores de facto a esas exigencias o no. La distinción entre ambos aspectos permite entender también la declaración kantiana de que "la benevolencia" sigue siendo "siempre deber, incluso hacia aquél que odia a los seres humanos, aquél, pues, a quien no se puede [...] amar" (MS, AA 06: 402.08-10). Sólo bajo un único escorzo se impondría la contradicción consistente en que aquél que no puede ser amado fuese sin embargo objeto de un deber de amor; también aquí se puede traer a colación de nuevo la vertiente positiva del misántropo estético en orden a remover la contracción. En lo que atañe a sus experiencias fácticas con seres humanos no puede reunir ningún amor de complacencia, dado que sus experiencias no han sido ya meramente indiferentes sino, desde el punto de vista estético, de disgusto a la vista de lo moralmente desagradable; y, no obstante, al misántropo estético se le atribuye la benevolencia filantrópica. Ésta ha de alimentarse, pues, de una fuente distinta de la facticidad de las experiencias y las reacciones sentimentales. Esta otra fuente es el pensamiento de que todo ser humano, en virtud de su propia razón pura práctica, produce en sí un ideal normativo sobre cómo haya de ser el ser humano. Es por ello que cualquiera puede ser amado, que cualquiera merece el amor de benevolencia. También por ello puede cada uno amarse a sí mismo, tal y como Kant expone en el parágrafo 25.

Ahora hemos de dilucidar aún (y, en la medida de lo posible, responder) la pregunta de si el amor, en el concepto de 'deber del amor' -amor que Kant ha caracterizado ya como "impropio"-, añade a su intelectualidad también un carácter emotivo. De que no resulta preciso tomar partido por 
una y contra el otro puede brindar un indicio el sentimiento del respeto, que, según Kant, es un sentimiento producido intelectualmente (cf., por ejemplo, KpV, AA 05: 78-79). También en el pasaje en que caracteriza expressis verbis como sentimiento al amor aquí tematizado se expresa una interacción de intelecto y facultad de placer y displacer: "El amor y el respeto son los sentimientos que acompañan el ejercicio de estos deberes" (MS, AA 06: 448.14 s.), donde con "deberes" se alude a los relacionados con los demás. La fuente de los deberes ha de ser, sin duda, la razón práctica. Esos sentimientos 'concomitantes' corresponden a la facultad del placer y del displacer; con la expresión 'acompañar' se mantiene, no obstante, en la vaguedad la índole de esa interacción. Ahora bien, se la puede especificar remitiéndose a un pasaje en el que Kant expone algo esclarecedor sobre la relación entre las facultades ligadas al deseo y al placer y displacer.

En la primera sección de la introducción general a la Metafisica de las costumbres, su tesis en torno a dicha relación señala que ésta se da siempre, de manera ininterrumpida, y que no hay deseo sin su correlato en el plano del placer o del displacer. "Con el deseo o la aversión se halla [...] enlazado en todo momento el placer o displacer, cuya receptividad se denomina sentimiento [...]" (MS, AA 06: 211.10 s.). El sentimiento necesariamente enlazado con el deseo se denomina aquí "placer práctico" (MS, AA 06: 212.12). ${ }^{4}$ De éste se dan, según Kant, dos tipos: o bien sería el placer de una representación sensorial -es decir, el placer de un ansia o inclinación patológica-, en primer lugar, a la cual seguiría entonces la determinación de la facultad de desear como efecto; o bien, en cambio, la determinación de la facultad de desear por parte de una representación intelectual sería lo primero y el efecto emotivo lo segundo. Un sentimiento producido de esta guisa podría denominarse "placer por espontaneidad". Kant lo llama "placer intelectual" (MS, AA 06: 212.29) o bien "inclinación independiente de los sentidos" (MS, AA 06: 213.08 s.). En todo ello, considera que el empleo de la expresión 'inclinación' ha de ser explicado ulteriormente. La habría empleado "para atender al uso lingüístico" (MS, AA 06: 213.04 s.). Esto equivale a decir que, tomada objetivamente, dicha expresión no resulta adecuada; hubiera resultado más que oportuno decir que la expresión 'inclinación' está empleada impropiamente. Y es que el placer producido intelectualmente no implica un ser atraído pasivamente hacia un objeto dado, sino que es un placer que tiende desde sí mismo, que se fundamenta en la actividad del intelecto y que se dirige a algo previamente proyectado, a saber, a una moralidad que ha de ser realizada en la figura de la felicidad ajena. A dicha inclinación en sentido impropio -inclinación intelectual o independiente de los sentidos, diferente de la inclinación sensiblela denomina finalmente Kant, de manera menos problemática, un "desear por puro interés de la razón" (MS, AA 06: 213.06 s.). Dicho interés estaría fundamentado "sobre principios puros de la razón" y su objeto sería aquello "que sólo puede ser objeto de un placer intelectual" (MS, AA 06: 212.31-213.06). Ahora bien, con "principio puro de la razón" de la facultad de desear sólo puede ser entendido uno delos imperativos de la razón pura práctica y con "objeto de un placer intelectual" sólo un fin que el imperativo requiere llevar a cabo.

El concepto de 'interés' es, en este contexto dado,el más significativo. Estar interesado en algo significa que al que se interesa le va algo en la existencia del objeto -a saber, bajo la forma de una implicación afectiva-. Según Kant, el interés es "la complacencia [...] que enlazamos con la representación de la existencia de un objeto" (KU, AA 05: 204.22 s.). Ahora bien, cuando se da un interés puro de la razón en un fin requerido por la razón práctica -lo cual, a su parecer, es el caso-,

\footnotetext{
${ }^{4}$ En su penetrante comentario sobre los parágrafos de la Metafisica de las costumbres que tratan los deberes del amor (\$§ 23-36), Dieter Schönecker (2013) diagnostica como problema irresuelto el hecho de que Kant introduzca el amor en el parágrafo 23 en el sentido de un sentimiento sin relación con el intelecto, para pasar en los siguientes parágrafos al amor, en cambio, como un concepto normativo sin relación con el sentimiento (cf. Schönecker 2013: 324). La presente contribución ha de ser considerada un tentativo de entender la noción de amor contenida en el concepto 'deber del amor' -no detalladamente desplegado por Kant y, por ello, altamente necesitado de explicación-, y de entenderla en tanto que tiene siempre una significación emotiva y se halla siempre sintéticamente enlazada con el concepto del deber -que siempre tiene una significación intelectual-, de manera que se trata siempre de una correlación libre de contradicciones. Por decirlo en el lenguaje de las facultades, dicha correlación descansa en el nexo ininterrumpido entre la facultad de desear -aquí la superior, la intelectual, que instituye un deber- y la facultad del placer y del displacer, que muestra una reacción emotiva.
} 
entonces se da una razón tendente, desde sí misma, hacia el fin prescrito, razón cuyo correlato emotivo es un sentimiento positivo. En cuanto inclinación no quiere la existencia del objeto a la manera de un mero querer intelectual, afectivamente indiferente, sino como forma de un querer amoroso que enlaza placer a la representación de la existencia del fin prescrito, un placer racional. ${ }^{5}$

A la luz de los resultados alcanzados, tomemos ahora aún en consideración el ya referido -y, en el marco de la doctrina kantiana de la virtud, chocante- mandamiento del amor al prójimo. El imperativo "ama a tu prójimo (al otro ser humano) como a ti mismo" (MS, AA 06: 451.29), que Kant afirma con carácter absoluto y que obviamente toma de la ética cristiana, no puede ser extrapolado a la ética kantiana sin modificarlo ni desambiguarlo, es decir, no se puede hacerlo en sentido literal; en efecto, dicho sentido no podría ser puesto en relación consistente con la tesis -por lo demás, sostenida- de que los sentimientos no pueden ser prescritos. Ahora bien, las explicaciones no literales de contenidos de la religión revelada son, según la hermeneutica sacra de Kant, legítimas. Puede darse que una tal "interpretación [...] nos parezca, con vistas al texto (de la Revelación), a menudo forzada, también a menudo verdadera"; lo que se precisa es sólo que la modificación explicativa “contenga en sí” algo "para la moralidad” (RGV, AA 06: 110.10-14).

Al margen del diferente fundamento de validez para el imperativo del amor al prójimo en una concepción y en la otra-Dios como fuente moral externa en la religión revelada, la razón pura práctica como una interna en el planteamiento autónomo kantiano-, en el punto que hemos introducido no se habría de entender el imperativo en el sentido literal que ordena directa e inmediatamente un sentimiento. Según la composición de lugar kantiana, la razón pura práctica se ordena, en primer lugar, a sí misma la benevolencia activa hacia los demás; a esta autodeterminación intelectual se le añade entonces 'como consecuencia' una reacción emotiva del lado de la facultad del placer y del displacer, e incluso el placer meramente práctico del amor al prójimo.

La desambiguación es especialmente necesaria respecto del viraje, en el marco de la fórmula del amor al prójimo, que a primera vista convierte el amor a sí mismo en la medida del amor al prójimo: "como a ti mismo". No se puede tratar aquí del tipo de amor a sí mismo que expresa, por lo demás, el uso kantiano; es decir, no puede tratarse de la relación afectiva consigo mismo del individuo menesteroso de felicidad en cuanto ser sensible. Antes bien, se habrá de tratar de una relación consigo mismo según el modelo de la relación con el prójimo que ya ha sido caracterizada, prójimo que en su calidad de perteneciente a la especie humana es destinatario de cumplimiento de deberes y que sobre la base del rasgo que distingue a dicha especie -a saber, la razón ético-prácticatambién es objeto de amor, del universal amor al prójimo. Según este modelo, el amor a sí mismo no es el individual del ser sensible, sino una relación afectivamente positiva consigo mismo sobre la base de la pertenencia a la especie distinguida con la razón ético-práctica. Para disipar la todavía posible apariencia última -también en esta explicación intelectualista- de primacía de la relación consigo mismo sobre las relaciones con el prójimo, aún se podría sustituir, a la postre,la expresión "como a ti mismo" por la de "y a ti mismo"; con ello se expresaría, de manera unívoca, la igualdad de rango.

Resulta evidente que sólo por medio de notables esfuerzos interpretativos -que quizá puedan parecer forzados- es posible integrar el imperativo del amor al prójimo, tomado de la ética cristiana, en la concepción kantiana de los deberes de la virtud. A la vista de esto resulta útil que la denominación de los deberes hacia los demás como 'deberes del amor’ pueda también ser legitimada sin recurrir a dicho imperativo.

\footnotetext{
${ }^{5}$ Las relaciones entre la facultad superior o inferior asociada al deseo, por una parte, y la facultad del placer y del displacer, por otra, han sido expuestas detalladamente por Thomas Höwing (2013). Como criterio decisivo a la hora de distinguir entre deseo sensible y racional, Höwing destaca con razón "si el principio práctico presupone o no un placer por el objeto deseado" (Höwing 2013: 180). Un placer que precede al deseo es, a su parecer, "ipso facto un placer sensible por lo agradable" (Höwing 2013: 146); en cambio, placer en cuanto "efecto de una actualización de la facultad superior de desear" es "placer por el bien" (Höwing 2013: 72).
} 
Como resultado de sopesar el concepto de amor original kantiano y sus especificaciones se puede establecer que el amor interpelado en el compuesto 'deber del amor' es el amor de benevolencia [amor benevolentiae]; éste no es amor propio de la inclinación en cuanto amor sensible, sino, como hemos visto, inclinación independiente de los sentidos y producida por la razón. No es, en cambio, amor de complacencia [amor complacentiae], que constituye, por su parte, la positiva reacción emotiva a experiencias morales satisfactorias -es decir, reacción afectiva a la perfección de otros-. Cuando se producen experiencias negativas con ellos, que se carezca del amor de complacencia y se dé el amor de benevolencia no entra en relación de contradicción; así se podría mostrarlo en la figura del misántropo estético, quien, a pesar de su misantropía 'estética' y al mismo tiempo, ha de ser pensado aún como 'filántropo'. De igual manera, el displacer que procede de la coacción a sí mismo no se halla en contradicción, en todo deber -y tampoco, pues, en el caso de todo deber del amor-, con el placer en la inclinación amorosa hacia otros seres humanos. Ese displacer es el displacer de la sensibilidad patológica por el sacrificio requerido en orden a la felicidad de los demás; el placer en la inclinación amorosa, en cambio, es producido intelectualmente y concierne a los demás seres humanos como seres de la especie, como meros seres humanos. ${ }^{6}$

En la 'deducción' del deber de promover la felicidad ajena no juega ningún papel sentimiento alguno y, por lo tanto, tampoco el amor. Por lo que respecta a su 'fundamentación', dicho deber es puramente intelectual y reconducible, pues, a la razón pura práctica. Lo prescrito por el deber no es el amor tampoco. El amor se da -concretamente, en cuanto amor de benevolencia- como 'consecuencia' de la autodeterminación intelectual en orden a los deberes hacia los demás, a saber, como efecto emotivo que acompaña por parte de la facultad del placer y del displacer, la cual es capaz de placer puro práctico. Si bien los deberes aquí tematizados no lo son ni 'por' el sentimiento de amor ni 'en orden al' sentimiento de amor, sobre la base de dicho carácter concomitante del sentimiento implicado -sobre la base, pues, de la participación de una tendencia independiente de la sensibilidadparece justificado, aun cuando no resulte estrictamente necesario, denominarlos así: 'deberes del amor'.

Traducción de Pedro Jesús Teruel

\section{Bibliografia}

HÖWING, Thomas: Praktische Lust. Kant über das Verhältnis von Fühlen, Begehrenund praktischer Vernunft, Berlín/Boston, De Gruyter, 2013.

RECKI, Birgit: "Wie fühlt man sich als vernünftiges Wesen? Immanuel Kant über ästhetische und moralische Gefühle", en HERDING, Klaus; STUMPHAUS, Bernhard (eds.): Pathos, Affekt, Gefühl, Berlín/Nueva York, De Gruyter, 2004, 274-294.

SCHÖNECKER, Dieter: "Duties to Others from Love", en TRAMPOTA Andreas; SENSEN, Oliver; TIMMERMANN, Jens (eds.): Kant's Tugendlehre, Berlín/Boston, De Gruyter, 2013, 310339.

: "Kant über Menschenliebe als moralische Gemütsanlage", Archivfür Geschichte der Philosophie 92, (2010), 175.

\footnotetext{
${ }^{6}$ Que los estados afectivos no han de ser monocromáticos, sino que pueden ser mixtos, lo expone Kant explícitamente en su análisis del sentimiento del respeto. También este sentimiento consiste, a su parecer, en una "humillación de la vertiente sensible" y una "elevación de la moral" (KpV, AA 05: 79.05 s.). Cf. a este respecto la contribución de Birgit Recki (2004).

${ }^{7}$ Universitat de València.
} 


\title{
Towards a new conception of metaphysics: Lambert's criticism on Wolff's mathematical method
}

\author{
GESA WELLMANN ${ }^{1}$
}

\begin{abstract}
This paper discusses Lambert's criticism of Wolff's mathematical method. I take this criticism to consist of two main aspects. Lambert tries on the one hand to revise Wolff's idea of fundamental concepts by providing what he takes to be a more profound theory of concepts.On the other hand, Lambert introduces postulates that regulate the connection between fundamental concepts and account for their application to experience. Both transformations allow Lambert to put forward a novel approach to metaphysics which is essentially oriented towards experience.
\end{abstract}

Key words: Lambert, Mathematical method, Metaphysics, Simple Concepts, Postulates

\section{Hacia una nueva concepción de la metafísica: la crítica de Lambert al método matemático de Wolff}

\section{Resumen}

Este artículo aborda la crítica de Lambert al método matemático de Wolff. Dicha crítica se desarrolla en torno a dos aspectos principales. Lambert intenta, por una parte, revisar la idea de los conceptos fundamentales de Wolff ofreciendo lo que considera una teoría de los conceptos más profunda. Por otra parte, Lambert introduce postulados que regulan la conexión entre los conceptos fundamentales y da cuenta de su aplicación a la experiencia. Ambas transformaciones permiten a Lambert presentar un tratamiento novedoso de la metafísica que está esencialmente orientado hacia la experiencia.

Palabras clave: Lambert, método matemático, metafísica, conceptos simples, postulados

\section{Introducción}

In his main philosophical works, the Organon (1764) and the Architectonic (1771), Johann Heinrich Lambert (1728-1777) points to what he conceives of as a mayor problem in Wolff's metaphysics. According to Lambert's analysis, the latter is of a merely formal nature. ${ }^{2}$ Wolff's metaphysics is, as Lambert sees it, formally correct; however, it does not ensure the validity of its principles beyond the logical domain. In the course of his examination, Lambert provides a diagnosis as to why Wolff fails to ensure such validity. In trying to establish metaphysics as a science, Wolff, according to Lambert, employs a defective method, namely what Wolff conceives of as the mathematical method. ${ }^{3}$ This method results from a translation of the strict demonstration in Euclid's Elements into a theory of

${ }^{1}$ Katholieke Universiteit Leuven, Bélgica.

${ }^{2}$ See Lambert, letter to Kant, February 3, 1766, (AA X: 64). See for a discussion of Lambert's criticism on the formality of Wolff's logic Blomme (2015: 108-111).

${ }^{3}$ As Wolters notes, unfortunately it is not known which books of Wolff Lambert possessed. The auction catalogue of Lambert's estate was only published in one copy, kept by the university library of Breslau and got lost during World War II. See Wolters (1979: 20). 
judgment and serves to determine and connect concepts and propositions. Briefly summarized, Wolff's mathematical method contains three steps: The truth of propositions that require a proof (theorems) is based on the truth of irrefutable propositions (axioms), which in turn are deduced from the correctness of concepts (definitions). ${ }^{4}$ Lambert agrees with Wolff's interpretation of Euclid's method for the most part. ${ }^{5}$ However, he argues that the tripartite argumentation of Wolff only suffices in so far as logic and mathematics are concerned. ${ }^{6}$ It covers, as Lambert sees it, the logical connection between concepts, but fails to provide rules for the application of these concepts to experience. ${ }^{7}$ In order to apply Euclid's theory to metaphysics, it must therefore, as Lambert holds, be reformed.

The aim of the present article is to examine how Lambert revises Wolff's mathematical method in this regard. Lambert's philosophical method has been partially researched in monographs such as Wolter's Basis und Deduktion (1979) and articles such as Basso's "Rien du mathématique dans la methodus mathematica wolffienne" (2008). ${ }^{8}$ However, in my view, previous studies have not sufficiently addressed the impact Lambert's reformed method exerts on his conception of metaphysics. In my interpretation, Lambert revises the mathematical method in view of a new conception of metaphysics, which is mainly concerned with the validity of its judgements in experience. Such a project shows a surprisingly modern approach to the justification of metaphysics as a science and anticipates, as I see it, Kant's notion of metaphysics. ${ }^{9}$ I will argue for this thesis in two steps. Firstly, I will examine Lambert's conception of simple concepts (1). Secondly, I investigate Lambert's theory of postulates (2). As I take it, both conceptions are the decisive tools Lambert uses in order to establish his new notion of metaphysics. Since Lambert's most detailed account of both conceptions can be found in his Architectonic, my paper will mostly rely on this work, while only briefly referring to Lambert's Organon and smaller philosophical writings.

\section{Simple concepts}

Lambert's criticism of Wolff's mathematical method departs from his criticism on those concepts that serve as the ultimate concepts in a definition, namely so-called 'common concepts', such as 'entity'. While Wolff takes these concepts to ground any further axioms of metaphysics, Lambert holds that they cannot account for any content of the axiom they are supposed to ground. Instead, Lambert

${ }^{4}$ Wolff (1733) states: "If I think carefully of everything that is contained in the mathematical method, I find these three main parts: (1) that all words [...] are explained by distinct and complete concepts; (2) that all propositions are proven by inferences that are properly connected among each other; (3) that no minor premise is assumed that is not proven before; such that the following propositions are connected with the preceding ones, as one connects an explanation with a preceding one by using words in the former that had been explained in the latter." See for a summary of Lambert's understanding of the mathematical method: Criterium veritatis, $\S 38$

${ }^{5}$ Organon,Aleth. $\$ 1$. Hereafter: Org. Lambert's notion of a system stands in the tradition of Aristotle, who influenced both Leibniz and Wolff. Lambert refers to this heritage in his Architectonic, $\$ \S 1-4$, while also pointing to what Lambert takes its weaknesses to be. Aristotle, as Lambert states, had arbitrarily gathered the basic concepts, while a metaphysical system would require a systematic deduction of the same.

${ }^{6}$ See Criterium veritatis, $\S \S 3,16,18-20$; the mathematical method, as Wolff proposes the latter, is, as Lambert states, sufficient with regard to geometry and logic since "the concepts [...] are simple and the figures lay before one's eyes" Criterium veritatis $\S$ 18. In logic, according to Lambert, one can identify the laws of thinking by observation, simply by perceiving one's own activity while thinking and concluding. See Lambert (1786), Die Theorie der Parallellinien, \& 5, hereafter: Parallellinien. With his example of parallel lines, Lambert points to the problem of Wolff's definitions. It is, according to Lambert, impossible to deduce a posteriori, i.e. abstracting from all given marks, an axiom of parallel lines. Such a deduction is impossible since geometrical precision and the possibility of drawing into infinity are excluded. See Parallellinien $\S 5$. See on this thesis also Dunlop (2009: $51)$.

7 This criticism is mainly directed at the foundation of Wolff's chain of proofs, namely his conception of definitions. Lambert rejects definitions as the ultimate starting point for metaphysics for two reasons. Firstly, a definition does not guarantee the validity of what is defined. Secondly, Lambert takes definitions to be only nominations (Benennung), which in themselves cannot prove the correctness of the axiom. Thus, in metaphysics, an axiom cannot be inferred from a definition. See for a similar criticism of the scholastic tradition: Kant (1781/1787) Critique of Pure Reason, A52/B76.

${ }^{8}$ See for comprehensive studies on Lamberts mathematical method: Bierbach (2001); Wolters (1979).

${ }^{9}$ See for a discussion of Lambert's notion of a system: Waibel (2007). See for a discussion of Kant's notion of a system: Critique of Pure Reason (A832/B861- A853/B881); on the same topic, see also: Henrich (2001:94, 101); Zöller (2001:60-72); Kambarte (1969: 109-111). See on the influence of Lambert on Kant's notion of ontology in general: Rivero (2014: 40); Watkins (2009: 231 f.). See on the influence of Lambert on Kant's notion of ontology with regard to the problem of definitions: Brandt (1995: 99). 
suggests taking so-called 'simple concepts' to be the fundamental concepts of metaphysics. In what follows, I will present Lambert's conception of such concepts against the backdrop of his criticism on Wolff (1.1) and discuss the consequences of his theory for his notion of metaphysics in a second step (1.2).

\subsection{Simple Concepts as Fundamental Concepts}

According to Wolff's logic, one important task of philosophy is to account for concepts that are a priori and can serve as the foundation for any empirical concepts. Traditionally the way to find such a priori concepts is by means of abstraction. ${ }^{10} \mathrm{~A}$ concept, according to Wolff's theory, is composed of particular [besondere] marks. The concept of 'horse', for instance, comprises marks such as having four legs, fur, mane, etc. ${ }^{11}$ These marks are empirically attained. In order to arrive at the general concept of mammal, one abstracts from the empirical origin of the marks and discards the marks that the concept shares with other concepts, for example fur. By examining the common marks of all concepts that contain fur, one arrives at the general concept 'mammal'. This concept, in turn, can again be compared with other concepts until one arrives at concepts that do not share any further marks with other concepts, for example 'entity'. ${ }^{12}$ Wolff denotes such concepts as common concepts [Gemeinbegriffe] and takes them to form the foundation of any philosophical system. ${ }^{13}$

Lambert's criticism of Wolff departs from this claim. More precisely, he rejects the idea that common concepts - that is to say most general concepts - can function as fundamental concepts. ${ }^{14}$ On the word of Lambert, the generality of a concept does not guarantee its validity. This means that one cannot, according to Lambert, exclude that a general concept is in itself a contradictory concept. ${ }^{15}$ One cannot do so because, in Lambert's eyes, any concept that has been arrived at by means of abstraction is a composed concept. ${ }^{16}$ For example, the concept 'entity' contains, according to Lambert, other concepts, such as 'the one', 'the true', 'the good'. Now, the principle of non-contradiction can be applied to such composed concepts only as a negative criterion of truth. If one finds a contradiction in a concept, one can be certain that the concept is impossible and thus not true. However, not finding such a contradiction does not guarantee that the concept is

${ }^{10}$ With respect to abstraction, Wolff refers to Aristotle and Leibniz. On the relation between Wolff and his predecessors as well as Wolff's deviations from Leibniz, see Ros (1989: 133-154).

${ }^{11}$ Lambert takes over this definition of a concept. In a letter to Kant, he states that a concept is "a combination of particular marks". Lambert'sletterto Kant, February 3, 1766, (AA X: 64).

${ }^{12}$ See Wolff (1713) Vernünfftige Gedancken von den Kräfften des menschlichen Verstandes $\S \S 1-114$. Hereafter: Kräfte des Verstandes. See on the equivocation of common concept [Gemeinbegriff] and general concept [Allgemeinbegriff]: Wolff (1729) De differentia intellectus systematici \& non-systematici, p. 255. Hereafter: De differentia.

${ }^{13}$ De differentia, $\$ 11$. I will not discuss the difference between general concepts (arrived at by means of abstraction) and universal concepts (arrived at by means of analysis) here, nor Lambert's rather obscure discussion of the importance of the method of abstraction for scientific cognition and its relation to the anatomic method. See for this discussion: Criterium Veritatis, § 3; Arch. $\$$ 7; Org., Dian. $\S \S 6,17,32-47,59,60,633,634,639,643$; Org. Aleth. $\S 9$. See for a discussion of Lambert's account of general concepts: Bierbach (2001: 138).

${ }^{14}$ Lambert's criticism is directed at the fact that by means of abstraction, Wolff arrives at nominal definitions whereas the contents of these definitions, i.e. the real definitions, are lacking. See Lambert, letter to Kant, February 3, 1766, (AA X: 64). Wolff, for his part, stresses the difference between nominal and real definition, while criticizing Descartes for having given only nominal definitions of the soul whereas as he, Wolff, provides the real definition of the latter. See Kräfte des Verstandes, $\S \S 48,54$. As Rivero (2014: 25) suggests, Lambert's criticism of Wolff's ontological concepts resembles Kant's criticism of Wolff's mathematical method in his Preisschrift (1763). Metaphysics, as Kant conceives of it, does not start from definitions but rather from experience (UD AA II, 276). Lambert changes his view on fundamental concepts slightly between his Criteriumveritatis and the Organon. See on this: Wolters (1979: 67).

${ }^{15}$ See Organon, Dian. $§ 110$.

${ }^{16}$ See Anlage zurArchitektonik, I, \& 14. Hereafter: Arch. Lambert's criticism is mainly directed against the concept of 'entity', which Wolff takes to be simple. In a letter to Holland, he states that the 'entity' is "the most composed [concept of all concepts] Because it contains in itself all kinds of fundamenta divisionum and subdivsionum", that is to say, apart from 'the one', 'the true' and 'the good', 'quale', 'quantum', 'numerabile', 'existentiae capax' and "countless others for which language don't even have words". Lambert's letter to Holland, April 12, 1765, (1782: 33). See also Organon, Dian. \$ 110. Bierbach (2001: 137, 138) stresses the important difference between Sache or Gegenstand and Ding, both referring to the Latin ens. Sache is a broader notion than Ding and can refer to a fact in general, whereas Ding refers to an entity (object). I will follow the Cambridge translation of the Critique of Pure Reason and translate Gegenstand with 'object' and Ding with 'entity'. As Bierbach rightly observes, Lambert seems to use Sache and Gegenstand mostly synonymously; hence I will translate Sache with 'object'. See for composed concepts: See Criterium Veritatis, $§ 7$. 
possible. Because a concept can be contradictory and thus impossible even if subjective reasons hinder us from finding such contradictions. ${ }^{17}$ Since common concepts cannot ensure that they are valid, Lambert concludes, they are not suitable for serving as fundamental concepts to metaphysics.

The principle of non-contradiction, according to Lambert, can only form a 'positive' criterion of truth if the concept 'cannot' contain any contradiction a priori. Concepts that meet such a demand are those concepts that contain only one mark, since "they do not contain any inner contradiction in themselves (an sich) since in order to contradict, several, or at least two parts are required". ${ }^{18}$ Concepts that contain only one particular mark are called 'simple concepts' and represent, as Lambert holds, the fundamental concepts of a scientific system. According to his argumentation in both the Organon and the Architectonic, they can be found in marks that cannot be dissolved into further marks. As they do not share a single mark with other concepts, Lambert describes simple concepts as "individual". ${ }^{19}$ This, as Lambert stresses, is in contrast to Leibniz and Wolff, whose general concepts only account for genera and species. ${ }^{20}$ However, Lambert abolishes neither Wolff's method of abstraction nor his theory of general concepts, but instead he seeks to ground the latter in what he takes to be more basic concepts.

Given that abstraction only leads to what Lambert considers as general concepts, simple concepts must, according to Lambert, be discovered by a different means. ${ }^{21}$ The method Lambert introduces - and which he refers to as "anatomic" - consists in demonstrating the "genealogy of concepts". ${ }^{22}$ As the name already implies, Lambert tries to investigate into the very origin of concepts and of simple concepts in particular. In line with Locke, he takes this origin to lie in experience. ${ }^{23}$ Since no contradiction can occur in a simple concept, its possibility is immediately given. Therefore, the possibility of simple concepts does not depend on experience. ${ }^{24}$ However, Lambert stresses that we, as finite beings, are dependent on experience in our search for simple concepts. ${ }^{25} \mathrm{We}$ experience, for instance, the impression of physical force or color, both of which Lambert denotes as simple concepts. ${ }^{26}$ Simple concepts are thus "taken from phenomena (Schein)" ${ }^{27}$

${ }^{17}$ See Criterium Veritatis, $\$ 10$

${ }^{18}$ Arch. \& 7.

${ }^{19}$ Lambert, letter to Kant, February 3, 1766, 22 [37], p. 47.

${ }^{20}$ Lambert, letter to Kant, February 3, 1766, 22 [37], p. 47. See on the relation between Leibniz and Lambert: Baxmann (2000: 243, 244); Siegwart (1988; XVII).

${ }^{21}$ See Lambert's letter to Holland, April 21, 1765, (1782: 23, 24, 32, 33). Normally, Lambert distinguishes between a 'synthetic' method, by means of which simple concepts can be discovered, and an 'analytic' method. Lambert mostly identifies analytic method and abstraction. The aim of this latter method is to find similarities among concepts. Such similarities result, as Lambert sees it, in nominal definitions, such that philosophy, if based only on this method, would be turned into a dictionary. In particular he criticizes Wolff for not discussing simple concepts such as space, time and duration. See Arch. § 13. However, Lambert is not always consistent with regard to his usage of 'analytic' and 'synthetic'. In Arch. 51 and Criterium veritatis $\S 37$, he claims for instance that the analytic method had lead him to the discovery of simple concepts.

${ }^{22}$ Arch. $\S 51$. See also Lambert's letter to Kant, February 3, 1766, (AA X: 65). With his anatomic method, Lambert tries to apply the mathematical method, which until then had been used only with regard to the connection of propositions, to concepts. See Arch. § 23. Just as propositions, so too concepts, according to Lambert, can be distinguished into fundamental and doctrinal ones [Lehrbegriffe], while the main task consists in discovering fundamental concepts. See Criterium veritatis $\S \S 25,37,38,39$. I will not discuss the division of all concepts into simple concepts of experience [Erfahrungsbegriffe] and doctrinal concepts [Lehrbegriffe] here in more detail. Lambert derives the latter from the former. The distinction between these concepts reflects the distinction between theorems and axioms. Lambert's theory of concepts in general is in line with Wolff's analysis of concepts in so far as composed concepts are reduced to simple ones by means of the "analytical method". See Criterium veritatis $\S 37$. Lambert highlights, however, the importance of clearly distinguishing between the classes of concepts (this point is mainly stressed in the Architectonic) in order to enable a pure derivation. See Criterium veritatis $\S 44$.

${ }^{23}$ See Org., Vorrede. See for the origin of simple concepts in sensibility also Arch. $\S 153$. Lambert conceives of his project as uniting elements of both Wolff's and Locke's philosophy. See Org. Vorrede. As Wolters (1979: 111) rightly points out, the view according to which concepts originate in sensual perception can also be found in parts of the scholastic tradition and, hence, Aristotle (cf. Thomas Aquinas' peripatetic axiom: "Nothing is in the intellect that was not first in the senses" De veritate, q. 2 a. 3 arg. 19).

${ }^{24}$ See Org., Dian. $\$ 656$

${ }_{25}$ According to Lambert's discussion in Org., Aleth. $\S 1$, simple concepts are internal to the mind [liegenim Gemüt $]$ and must be activated by means of experience. On this, see Leibniz's introduction to his Nouveaux Essais, which Lambert did not know, since they were published only in 1765 .

${ }^{26} \mathrm{Arch}$. $\$ 46$. In Org. § 234 a, Lambert states furthermore that God has to be understood as the source of all simple concepts. The function of theology is, according to Lambert, to prove this thesis. 
Lambert discusses the advantages of the empirical origin of simple concepts in his correspondence with Kant. Discussing Kant's Dissertation, in what is arguably his most influential letter to Kant, Lambert agrees with Kant on the difference between the sensual and the intellectual sources of our cognition. For Lambert, it is clear that

human cognition [...] falls into the old [distinction between] phaenomenon and noumenon and, according to this distinction (Einteilung) arises from two entirely distinct and as it were heterogeneous sources, such that what comes from the one source can never be deduced from the other. ${ }^{28}$

However, against Kant's approach in his Dissertation, Lambert stresses the importance of concepts that are taken from experience, since "in ontology, it is very useful to analyze as well those concepts that are taken from appearance, since their theory must be applied to phaenomenis again". ${ }^{29}$ In other words, Lambert holds that simple concepts must be discovered by means of experience because he aims to avoid Wolff's problem, namely ending up with a purely formal set of ontological concepts. ${ }^{30}$ If ontology is based on concepts that are taken from experience, on the other hand, Lambert is confident that it can apply its principles to experience again. ${ }^{31}$

It should be stressed that this approach is based on a notion of a priori that differs from that of Kant and others. ${ }^{32}$ 'A priori' in Lambert's sense does not exclude empirical input. Simple concepts have an empirical character, since it is only by means of the anatomic method that they are discovered. Their legitimacy, however, is not based on experience but rather on their having only one mark. ${ }^{33}$ As they are valid independently of experience, they are, as Lambert states, a priori. ${ }^{34}$ Thus, Lambert is clearly sensitive to the problem that metaphysics should take experience into account while at the same time remaining a science of a priori truths rather than of empirical concepts. In line with this, the fact that simple concepts are discovered by means of experience does not, in Lambert's view, limit their scope to the empirical world. Rather, simple concepts can be transferred to the socalled intellectual world, i.e. to the understanding and the will: "The designations of objects in the intellectual world are taken from the objects in the sensual world (Körperwelt)". ${ }^{35}$ If I experience physical force by lifting a weight, for example, I can extend the concept of force to the intellectual world, such that we can speak of the force of the understanding or the force of the will. ${ }^{36}$ In such a way, simple concepts can be freed from a merely sensual context. ${ }^{37}$

${ }^{27}$ Arch. $§ 43$.

${ }^{28}$ Lambert's letter to Kant, October 13, 1770, (AA X: 105).

${ }^{29}$ Lambert's letter to Kant, October 13, 1770, (AA X: 108). Kant will, as Beck (1969: 127) argues, develop his theory of space and time in virtue of Lambert's objection in this letter.

${ }^{30}$ With regard to Lambert's conception of empirically given objects and the importance of the latter for metaphysics as an a priori science, I hold a position between two authors: against Wolters (1979), I hold that Lambert was aware of the problem of an object in its empirical sense and its fundamental difference to the object of logic. Against Beck (1969), I hold that Lambert was not aware of the dimension of this differentiation, as he did not reflect on the consequences of his theory.

${ }^{31}$ Wolters (1969: 76), who argues that Lambert's aim was to establish conditions for the natural sciences, interprets Lambert's notion of experience as being "lebensweltlich". Lambert's simple concepts, according to Wolters (1979: 73-82), are taken from a notion of experience that coincides with historical knowledge, i.e. common knowledge based on observation. Although I agree that Lambert puts forward such a notion of experience, I take it to be inadequate precisely in the case of simple concepts. There, Lambert explicitly refers to an individual sensual impression. Cf. Arch. § 29 as well as Org. Dian. § 551.

${ }^{32}$ See on the different use of "a priori" in Lambert and Kant Wolters (1979: 77-79).

${ }^{33}$ See Arch. § 24; according to Wolters, Lambert introduces a new meaning of a priori insofar as he uses the term to describe a scientific context (in contrast to the prior syllogistic use of a priori). See on this Wolters (1979: 77).

${ }^{34}$ It is difficult to decide whether Lambert was aware of the special status of simple concepts. Wolters (1979: 77) explains the status of simple concepts by pointing to Lambert's notion of a priori, which does not exclude any empirical impact. Furthermore, Wolters (1979: 56; 76-81) stresses that Lambert's concept of experience (and consequently his notion of 'empirical') differs from that of what is commonly understood as empiricism. In general, I agree with this interpretation. However, as has become clear from what I have discussed above, Lambert does distinguish between what could be called "empirical" and "a priori" cognition, for instance in the letter of 1770 to Kant on human cognition that I just cited. Beck (1969: 127) takes this letter to be of utmost importance precisely with respect to the influence of Lambert on Kant with regard to the problem of the relation between a priori concept and empirically given objects.

${ }^{35}$ Arch. $\S 29$, see on that also Arch. $\$ 97$.

${ }^{36}$ See Arch. § 29; Org. Dian. § 551.

${ }^{37}$ Lambert only transfers certain simple concepts to the intellectual world, namely those that he calls 'fundamental concepts'. 
In the Organon and the Architectonic, Lambert presents a loosely collected list of simple concepts that correspond for the most part with Locke's simple ideas. ${ }^{38}$ These simple concepts consist first of all in eight so-called simple fundamental concepts, namely solidity, existence, duration, extension, force, movability, unity and identity. ${ }^{39}$ Next to these fundamental concepts, other simple concepts are taken "from sensual appearance (Schein)". These include color and sound, verbs, adverbs, prepositions and conjunctions, that is, concepts such as 'being', 'equal', 'through' and 'because' ${ }^{40}$ Since Lambert takes all of these concepts to be independent from experience in the sense discussed above, these concepts serve as the foundation of all a priori cognition in the Organon and the Architectonic.

\subsection{Simple Concepts and Ontology}

Having demarcated a group of simple concepts, namely fundamental concepts, from the rest, Lambert takes them alone to be suitable to provide the foundation of scientific cognition. Simple concepts in general are a priori and are used in a priori cognition. Simple fundamental concepts, on the other hand, serve as the foundation of 'all' a priori cognition altogether since any composed concept that is used in scientific cognition can be deduced from a combination of fundamental concepts. ${ }^{41}$ In order to give an overview on this latter function, Lambert designs what he calls the 'table of correlations' ${ }^{42}$ This table, as Lambert states, denotes a system that presents the foundation of all a priori cognition. It does so by showing two things. Firstly, it shows how fundamental concepts can be combined:

Since simple concepts are the first foundation of our cognition, and since everything of composed concepts, inasmuch as we represent the latter [...], can be dissolved into simple concepts; simple concepts, separately and combined among each other, form a system which necessarily contains all first foundations of our cognition. ${ }^{43}$

Secondly, the table shows which combinations of fundamental concepts underlie which a priori science. ${ }^{44}$ Unfortunately, it is not quite clear how Lambert arrives at the relations between concept and science. He only casually remarks: "Furthermore, every column presents a special theory and science". ${ }^{45}$ Lambert differentiates sixteen of these a priori sciences, among others geometry, ontology and dynamics, which he arranges as follows: ${ }^{46}$

${ }^{38}$ See Arch. § 9; cf. Org., Dian. § 646, Aleth. § 38; In his Organon, Dianologia § 653, Lambert explicitly refers to Locke's distinction between simple concepts (taken from immediate sensation) and general concepts (arrived at by abstraction, viz. the understanding). Lambert does not exactly follow Locke`s division of simple concepts into four classes. In the table of Locke's concepts that follows I highlight those concepts that Lambert considers to be simple concepts, or as Locke puts it, simple ideas: 1) ideas which come through one sense organ (color, sound, smell, etc.);2) ideas which come through more than one sense-organ (extension, shape and motion, solidity); 3) ideas which come from an inner faculty (thinking, volition, memory, judgment, knowledge, faith). This does not imply that those ideas are a priori. The observation of our mental operations presupposes that the mind operates with contents given by experience; 4) ideas which come from both external and internal faculties (pleasure, pain, being, power, unity and succession). Cf. Locke, An Essay Concerning Human Understanding, especially book II and III. Accordingtohis own statement, Lambert readLocke'sEssay Concerning Human Understanding: "I read Wolff, Von den Kräften des menschlichen Verstandes, Malebranche, Von der Erforschung der Wahrheit, Locke, Gedanken vom menschlichen Verstande. The mathematical sciences, in particular algebra and mechanics, provided me with distinct and thorough examples in order to confirm the rules I learned." Lambert, letter of 1750, cited in: Krienelke (1909: 11). As Wolters (1979: 19, 56) argues, it is improbable that Lambert knew the works of Hume.

${ }^{39}$ See Arch. $\$ 49$. In his enumeration, Lambert lists 10 fundamental concepts, limits their discussion in the Architectonic, however, to eight, since "the first [namely consciousness] can be found in any [a priori science], the latter [namely the will] on the other hand has its own object, namely the good and thus belongs particularly to agathology or the doctrine of the good". Arch. § 52 .

${ }^{40}$ Arch. $\$ 46$.

${ }^{41}$ See Org. Dian. § 653; See also Arch. § 23; Org. Dian. § 623.

${ }^{42}$ Arch. $\$ 71$.

${ }^{43}$ Arch. $\$ 74$.

${ }^{44}$ See Arch. $\$ 53$.

${ }^{45}$ Arch. $\$ 71$.

${ }^{46}$ See Arch. \& 74. Legend:

the fundamental concept (der zum Grunde gelegte Begriff)

a concept that is necessarily connected to the fundamental concept

the object of the fundamental concept

the compared concept 


\section{Korrelate der Grundlehren}

\begin{tabular}{|c|c|c|c|c|c|c|c|c|c|c|c|c|c|c|c|}
\hline & 1 & 2 & 3 & 4 & 5 & 6 & 7 & 8 & 9 & 10 & 11 & 12 & 13 & 14 & $5 \mid 1$ \\
\hline Solidität & + & - & $\approx$ & $\because$ & 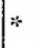 & $=$ & - & - & & & - & $-1=$ & $=$ & $=$ & - \\
\hline Existenz & - & - & - & - & & * & - & - & & & & & & & \\
\hline Dauer & - & - & - & - & & $=$ & $\approx$ & $*$ & * & & & - & & $-1=$ & $==$ \\
\hline Ausdehnung & - & - & $=$ & & - & & & - & & $\%$ & \% & * & & - & $=$ \\
\hline Kraft & + & - & - & - & - & $=$ & - & & & & - & - & * & * & - \\
\hline Beweglichkeit & - & - & - & & & & & & & & & - & & -1 & $\%$ \\
\hline Einheit & - & $*$ & - & & - & $=$ & & & - & - & - & - & - & - & - \\
\hline Identität & $*$ & - & - & & & $=$ & & & & & & & & & \\
\hline & 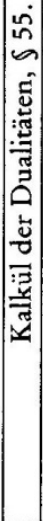 & 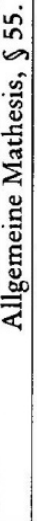 & 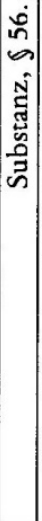 & 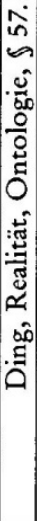 & 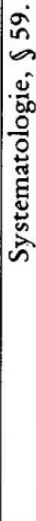 & 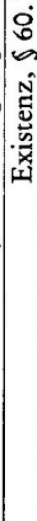 & 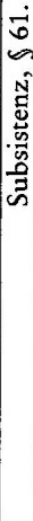 & 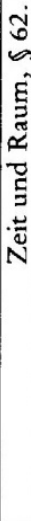 & 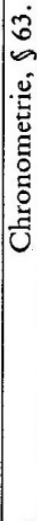 & 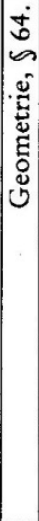 & $\begin{array}{c}10 \\
0 \\
0 \\
0 \\
0 \\
\tilde{y} \\
\end{array}$ & 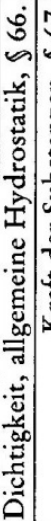 & 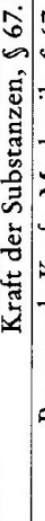 & 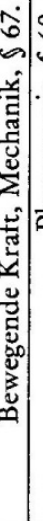 & 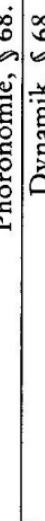 \\
\hline
\end{tabular}

As I see it, this table nicely illustrates Lambert's conception of metaphysics in general and his conception of ontology in particular. Firstly, it affirms what I argued for above, namely that for Lambert, ontology is based on a combination of simple concepts taken from experience. More precisely, Lambert bases his ontology on the simple concept of solidity. I take the last part of the Architectonic to develop such an ontology, more precisely the second, third and fourth part, in which Lambert discusses the ideal', 'the real' and 'magnitude'. ${ }^{47}$ In these chapters, Lambert investigates the fundamental concepts, which he established in the first part of the Architectonic, with regard to their application in philosophical cognition of solid objects. ${ }^{48}$ This is particularly remarkable with regard to

In total, Lambert refers to 16 sciences as 'a priori sciences', all of them based on the eight fundamental concepts. In his Criterium Veritatis, however, Lambert presents two forms of immediate truths apart from fundamental concepts: the Cartesian cogito and the sensation of harmony with respect to systematically structured cognition (§ 79). In the Organon and the Architectonic, these three elements can be found as well. However, as Lambert focuses, except for a few exceptions, on a discussion of simple concepts, I will only take the latter into account. Even though Lambert admits that the table might contain more than 16 columns, he takes the foundational science to be constant and evident. Future generations could add combinations of concepts, the simple concepts, however, are unchangeable, and the possibility of the combination is thus limited (Cf. Arch. $\S \S 54,69$ ).

${ }^{47}$ See Arch. Vorrede, X and XVI. See also Arch. $\$ 161$. I take Lambert's Architectonic to provide an alternative propaedeutic to a future system of metaphysics, which Lambert planned to establish. See Über die Methode, § 53. In this sense, the Architectonic fulfills a twofold function: the first part of the Architectonic provides a doctrine of method; the second part delivers parts of Lambert's system. See on Lambert's conception of architectonic: Manchester (2003: 187-207). See on the preparatory role of Lambert's Architectonic in particular: Wellmann (2017).

${ }^{48}$ The second part of the Architectonic, which I take to deal with Lambert's ontology, is based on another table, the so-called table of axioms. Solidity is primarily ascribed to impenetrable bodies (see Arch. $\S \S 48,57,90,92-94$ ). However, Lambert transfers the concept also to the intellectual domain: "in such a way, the concept of solidity becomes transcendent and one calls a cognition 
the former function of ontology. Traditionally, the three objects of metaphysics, God, the soul and material things constitute the threefold division of special metaphysics in theology, psychology and cosmology. On this account, ontology serves as a fundamental science to these objects by treating an object in general. ${ }^{49}$ Lambert, on the other hand, releases ontology from this preparatory function for special metaphysics. Ontology is not defined as a fundamental science 'for' some discipline anymore, but claims for itself the status of an independent science of that which is solid. ${ }^{50}$

Affirming this new role of ontology, the table shows secondly that Lambert did not intend to include the disciplines of special metaphysics - rational psychology, cosmology and theology nor, for that matter, logic or agathology in his system of a priori sciences. Even though Lambert takes all of these disciplines to be based on simple concepts, apparently, he considers them to be of a different kind than the a priori sciences mentioned in the table. ${ }^{51}$ Since he describes the table as giving an overview on all a priori sciences, it is very likely that Lambert did not consider them to become sciences at all. To ontology, on the other hand, Lambert ascribes a similar status as to any of the other sciences that are listed in the table. ${ }^{52}$ Thus, Lambert seems to be optimistic that ontology could, by means of his theory of simple concepts, become a proper science. However, on the basis of simple concepts alone, ontology, as Lambert sees it, cannot be established. As the following section aims to show, Lambert takes the application of these concepts to experience to be an equally important element.

\section{Postulates}

The previous section discussed a first aspect of Lambert's criticism of the mathematical method, such as it is advocated by Wolff. This criticism consists in rejecting definitions as the basis of ontology. With his version of simple concepts, Lambert tries to avoid what he understands as purely formal cognition. ${ }^{53}$ The current section discusses a second point of criticism, which builds on this thought. Wolff's system, according to Lambert, consists in the analysis of, and connection between, general concepts. This connection is regulated by logical principles such as the principle of non-contradiction. Lambert, on the other hand, tries to base these merely logical principles in more basic ones to which he refers as postulates and that he takes to arise directly from simple concepts. Hence, Lambert criticizes not only the formality of nominal definitions, but also the exclusive use of logical principles. In order to elucidate this claim, I will first examine how Lambert conceives of postulates in general (2.1) and then clarify the postulate's function with regard to ontology (2.2).

\subsection{Postulates as Principles}

As argued above, the ultimate function of simple concepts is to ground those sciences that Lambert notes in his table of correlations. ${ }^{54}$ Building up a science on the basis of such simple concepts requires, as Lambert states, that they are connected. The problem of connection arises due to the specific nature Lambert ascribed to simple concepts:

'solid' if it is not only dreamt but real, scientific and thorough (gründlich) and if its inferences and conclusions are necessary" Arch. $\S 93$. As far as I can see, there is no indication though that the table of axioms deals with such a broad notion of solidity.

${ }^{49}$ See Arch. $\S 19$. See on the structure of metaphysics in the $17^{\text {th }}$ and $18^{\text {th }}$ century: Vollrath (1962).

${ }^{50}$ See on the founding function of ontology in scholastic philosophy: Vollrath $(1962: 263,267)$

${ }^{51}$ TheOrganon and the Architectonic treat the axioms of special metaphysics though. Cf. Org. Aleth. $\S 247$. On agathology, see Arch. § 52; on special metaphysics, see Org. Aleth. $\S 234$ a, 247; on logic see Über die Methode.

${ }^{52}$ See on the difference between ontology and special metaphysics: Org. Aleth. § 195.

${ }^{53}$ Lambert, letter to Kant, February 3, 1766, (AA X: 64). Rivero (2014: 33) argues that Lambert's criticism of the formality of Wolff's architectonic refers to the fact that Wolff's principles only refer to ontology, while Lambert's principles take other a priori sciences into account as well. Although I agree that Lambert's architectonic is not only designed to prepare ontology, I take such an explanation to blur Lambert's original intention behind avoiding formality: namely to prepare an ontology that can be applied to experience. See on this subject: Lambert's letter to Holland, April 21, 1765, (1782: 31), as well as: Arch. §§ 18, 239.

${ }^{54}$ See Arch. Vorrede VII. 
It is important to closely determine the proposition [...] of the absolute (uneingeschränkt) manifoldness of composed concepts and entities, since simple concepts are different as such and therefore provide the first grounds of heterogeneity (Ungleichartigkeit) [...]. As they can be put together, determined and connected among each other, the question arises in how far one has a choice to maintain certain or several determinations, connections and relations. ${ }^{55}$

As the quote makes clear, simple concepts are inherently heterogeneous [an sichverschieden] and it is their combination that serves as the first ground of any heterogeneity [Ungleichartigkeit] and 'manifoldness' of complex concepts. ${ }^{56}$ The theory of simple concepts is therefore in need of a principle that guarantees that the simple concepts can be applied to any given manifold in a valid way. ${ }^{57}$ This task cannot be fulfilled by logical principles alone, as Wolff proposes. Lambert elaborates on the deficiency in this regard in his second letter to Kant. ${ }^{58}$ In 1766, Lambert draws Kant's attention to the question as to "whether and to what extent the cognition of the form leads to cognition of the matter of our knowledge". ${ }^{59}$ On Lambert's interpretation, logical principles, such as the principle of non-contradiction, cannot guarantee the validity of any judgements. ${ }^{60}$ The application of a simple concept to a given manifold can, as Lambert sees it, only be executed by a principle that is not purely formal. And it is, according to Lambert, only postulates that fulfill this requirement. Since postulates are laws that stem from simple concepts, they differ in origin from logical laws. It is, as Lambert writes to Kant, "matter that gives postulates"; or, as the Architectonic states, postulates are "taken from the objects themselves". ${ }^{61}$

In line with his general approach, Lambert claims to follow Euclid here. As he remarks in his first letter to Kant, the theory of postulates is just what is missing if one is to strictly following the Euclidian method: "Wolff implemented about half of the mathematical method into philosophy. If the other half is implemented as well, we gain what we desired". ${ }^{2}$ Trying to provide this 'other half', Lambert's theory of postulates constitutes a second addition to Wolff's tripartite version of the mathematical method: After having introduced simple concepts which are supposed to precede definitions, Lambert presents postulates which are supposed to precede axioms. While Wolff, according to Lambert, tries to justify the axiom directly from a definition, it is clear from Euclid's theory that an intermediate step is required: Between definitions and axioms, postulates must be inserted. Thus, Euclid's elaborations on geometrical figures show, according to Lambert, that "every definition, before being proven, is an empty hypothesis". ${ }^{63}$ The Euclidean definitions are denotations, while their proofs can only be provided by a postulate. Thus, for example, the definition "a line is a

\footnotetext{
${ }^{55}$ Arch. $\$ 156$.

${ }^{56}$ See on the problem of the relation between simple and composed concepts also Lambert's letter to Kant from February 3, 1766 , (AA X: 65). See also Arch. $\S \S 23 ; 122 ; 135$, where Lambert stresses the importance of the combination of simple concepts with regard to composed concepts.

${ }^{57}$ See Arch. $\S 135$.

${ }^{58}$ Further passages where Lambert discusses the defects of Wolff's method are: Lambert's letter to Holland (1782: 29), see also Org. Aleth., § 242; Arch. § 15.

${ }^{59}$ Lambert's letter to Kant, February 3, 1766, (AA X: 64). See also: Lambert's letter to Kant, November 13, 1765, (AA X: 52); Lambert's letter to Kant; letter to Holland, 1782, (AA X: 31). See also: Arch. $\S \S 14,18,239$. I am simplifying Lambert's argument here, which runs as follows: the form, i.e. logical principles, lead us to simple concepts, which provide us with content. If this content is to be used in a science, simple concepts must be combined. This takes place by means of postulates. Cf. Lambert's letter to Kant, February 3, 1766, (AA X: 46)

${ }^{60}$ The problem Lambert formulates here might recall the criticism Kant puts forward against scholastic philosophy. Indeed, some scholars, such as Beck, have pointed out that Lambert's criticism of the purely formal character of metaphysics had a great impact on Kant's development of transcendental philosophy. (Cf. Brandt (1995: 98, 99), Beck (1969: 127), Rivero (2014: 33)). These scholars stress the impact Lambert might have had in pointing Kant to the problem of form and matter. Furthermore I take it that Lambert might have had an influence in justifying the use of 'non-classical' principles, such as postulates or schemata, by pointing to their ability to apply a concept.

${ }^{61}$ The full quote reads: "the form gives principles, the matter axioms and postulates". Lambert's letter to Kant, February 3, 1766 , (AA X: 65). See on the relation between matter and axioms in contrast to principles that only refer to the definition of a thing: Arch. $\$ 43$.

${ }^{62}$ Lambert'sletterto Kant, November 13, 1765, (AA X: 54)

${ }^{63}$ Die Theorie der Parallellinien, $\S 6$. See on Lambert's criticism on Wolff with respect to lacking postulates: Arch. $\S \S 12$, 15. In the context of this paper, I cannot examine in how far this criticism is justified. See on a discussion of this subject: Basso (2008: 109-121).
} 
breadthless length" must be proven by the postulate "to produce a finite straight line continuously in a straight line". ${ }^{64}$ In Euclidean geometry, one must first draw a single line in order to prove the applicability of a general rule that applies to all lines, that is to say all special cases. ${ }^{65}$

Lambert takes the postulates he finds in Euclid to differ from any other principles because of their "practical" nature. ${ }^{66}$ Whereas Wolff, on Lambert's account, only used descriptive laws in order to connect his fundamental concepts, he attributes a prescriptive task to the postulates. In his Elements, Lambert remarks, Euclid proves that a line is possible by demonstrating that one can 'produce' a line. ${ }^{67}$ The conclusive force of postulates lies thus not in the definitions of the concepts that are contained in the postulates but rather in the fact that they force the reader to follow the instructions of a certain problem in such a way that she understands the truth of the proposition by solving the problem. ${ }^{68}$ Postulates, as Lambert conceives of them, are propositions that are proven by experience, which "can be renewed any time, as often as one doubts their validity". ${ }^{69}$

Applied to the problem of metaphysics, this means that postulates are required to legitimately combine fundamental concepts, because they determine the extension and very possibility of a fundamental concept. Just as we can construct any line in geometry once we know how a line is constructed, one must, as Lambert states, find general rules for metaphysics that allow for the construction of a composite concept or axiom. ${ }^{70}$ What this means in particular in the Architectonic is that each of the eight fundamental concepts is accompanied by a certain number of postulates. After having introduced the "enumeration and immediate comparison" of simple concepts in the first part of the Architectonic, Lambert thus elaborates on the postulates that follow from such a comparison. ${ }^{71}$ Accordingly, the Architectonic formulates postulates of each simple concept on the basis of the possible relation of this concept to others, as outlined in the table of correlations. So, for instance, as we see in the tenth column, Lambert combines the concept of extension with the concept of unity. One of the postulates Lambert infers from extension reads: "Space has no particular unity". ${ }^{72}$ Without this postulate, metaphysics would have to examine the concept of extension in single case studies. ${ }^{73}$ By means of this postulate, however, philosophy can apply the concept 'extension' to any case.

\footnotetext{
${ }^{64}$ Euclid Elements, Book I, Definition 2; Book I, Postulate 2.

${ }^{65}$ Lambert differentiates between postulates and axioms in the following way: Postulates determine the general and unconditioned possibilities to form a complex concept on the basis of a simple one, while axioms indicate the limits of such possibilities (See Arch. § 13). While axioms build forth on the general possibilities determined by the postulates, postulates themselves are supposed to guarantee that the general rule (posited by the enunciation) can be applied to all particular cases. However, Lambert does not consistently differentiates between postulates and axioms, such that at other passages postulates seem to determine the limits of the possibility of a concept (Cf. Arch. $\S 76$ and in contrast $\$ 123)$. In is criterium veritatis, Lambert differentiates between axioms and postulates, however, the difference is not quite clear neither.

${ }^{66}$ Arch. $\S 18$. See on the practical character of postulates Hintikka (1967: 361). Dunlop (2009: 52) argues that the practical character of postulates in Lambert's Architectonic corresponds with Wolff's conception of the will. This thesis could be strengthened by consulting the Systematology which Dunlop does not take into account. The Systematology implies that postulates are not only practical since they force the reader to understand a rule by means of the example of a special case but also, referring to Leibniz, since the will is involved in connecting subject and predicate. See Systematology, $\S 21$.

${ }^{67}$ See for the practical implication of postulates: Wolters $(1979: 45,88,90)$. Wolters elaborates here on the role of forces in Lambert's notion of postulates. Since postulates determine which act can successfully be executed, they imply the use of forces. Although I take forces to play an important role in Lambert's general view of ontology, I can't discuss them here in greater detail. In a letter to Holland, Lambert elucidates how he conceives of the application of the Euclidean postulates and axioms in geometry to metaphysics. (See Lambert's letter to Holland, April 21, 1765, (1782: 28, 29, 31, 32). See for the description of the mathematical method: Hintikka (1967: 361).

${ }^{68}$ Laywine points out that by showing the possibility of a figure, the possibility of its concepts is given at the same time, since, at least in geometry, one has to know what one is doing before doing so. See Laywine (2010:119). Dunlop (2009: $52 \mathrm{f}$.) develops the thesis that with such a theory, Lambert tried to defend a system based on fundamental concepts against skepticism.

${ }^{69}$ Über die Methode, $\$ 21$.

${ }^{70}$ See Lambert's letter to Hollande, April 21, 1765, (1782: 29); Org. Aleth. § 242.

${ }^{71}$ Arch. $\$ 76$.

${ }^{72}$ Arch. $\$ 79$. Lambert discusses the postulates of every simple concept except for identity. See on the general possibility of postulates: Laywine (2010: 121). Dunlop (2009: 63) states that postulates provide fundamental concepts with their objects. However, I take Lambert's text not to justify such a reading.

${ }^{73}$ As postulates determine the possibility of a simple concept, they are, as Lambert states, to be understood as soon as the respective fundamental concept has been understood. Cf. Org. § 164.
} 


\subsection{Postulates and Ontology}

Lambert does not discuss the theory of postulates with regard to any science in particular, but takes it to be valid for all sciences that are mentioned in the table of correlations. On the basis of Lambert's statement that postulates "lay the ground for any scientific cognition", some commentators, such as Wolters, have argued that postulates (and for that matter Lambert's enterprise as a whole) only aim at guaranteeing the conditions of (natural) sciences in general. ${ }^{74}$ By contrast, I hold that Lambert was first and foremost interested in the possibility of saving the scientific status of ontology. I take this claim to be justified since Lambert discusses the advantages of postulates mostly in the context of his ontological project. Next to the above discussed function of postulates to secure the application of axioms, Lambert stresses the advantage postulates offer by comparing the results of several sciences. In this regard, Lambert highlights particularly the role of ontology. The results from ontology should, according to Lambert, serve as the basis for other sciences and conversely. ${ }^{75}$ Accordingly, the last parts of the Architectonic are larded with examples from ontology's influence on exact sciences. For example, in the fourth part - which deals with 'magnitude' - Lambert repeatedly stresses the importance of the mutual exchange between philosophy and mathematics. ${ }^{76}$ On my interpretation, such a discussion helps Lambert to stress on the one hand that ontology, as a science, improves by taking the results of other sciences (and especially of natural sciences) into account. On the other hand, it allows him to emphasize the necessity of a discipline such as ontology by highlighting its indispensable impact on other sciences.

It is with this twofold aim in view that Lambert discusses not only the relation between ontology and other sciences; but also between ontology and our everyday experience in the world. ${ }^{77}$ With his theory of postulates Lambert hopes to achieve what he promises in his theory of simple concepts - namely limiting the scope of ontology to what he calls "everyday life" [imgemeinen Leben $].{ }^{78}$ Ontology should not only concern logical truths, but should be applicable to the world. ${ }^{79}$ In addition to what has been argued above - namely that Lambert's ontology is based on the simple concept of solidity, instead of examining objects in general - the theory of postulates enables this role of ontology by allowing for the application of ontology's concepts to experience or, for that matter, historical cognition. This allows Lambert to put forward a new criterion for success with regard to ontology: Ontology no longer aims to support the claims of special metaphysics. Rather, it is supposed to provide principles that are ultimately applicable in experience. ${ }^{80}$ Lambert's new conception of ontology is mirrored in the modest expectations Lambert holds with regard to metaphysics in general. He states throughout the Organon and the Architectonic that the philosopher should be content with the limited results his research delivers. Reflecting upon his own work, Lambert rightfully stresses "the difference between former ontologies, including their order, and the present one". ${ }^{81}$ By means of his revised mathematical method Lambert thus hopes to have established

${ }^{74}$ Lambert's letter to Kant, February 3, 1766, (AA X: 66). Wolters (1969: 15-28).

${ }^{75}$ See Arch. Vorrede, X.

${ }^{76}$ Lambert stresses that both ontology and mathematics should exchange their methods and results, while he points at the same time to the importance of differentiating between them. See Arch. $\S \S 679-685$; on the relation between ontology and mathematics see in particular Arch. $\S \S 681$ and 683.

${ }_{77}$ The question concerning the possibility of metaphysics as a science and in particular the method to be used in this regards experienced an upswing in Lambert's times. The prize essay contest, organized by the Berlin Academy of sciences shortly after Lambert's death, exemplifies the wide interest in this issue. For a detailed discussion of the development of the systematic method see Engfer (1982).

${ }_{78}$ Arch. Vorrede, IX.

${ }^{79}$ See Arch. Vorrede, IX.

${ }^{80}$ Lambert's new criterion of success for ontology forces him to provide a propaedeutic work which clarifies under which conditions a priori principles are applicable to experience. I take this propaedeutic to be delivered in his Architectonic. See on a discussion of the Architectonic and its propaedeutic function: Wellmann (2017).

${ }_{81}$ Lambert (1787), Logische und philosophische Abhandlungen, 414. Lambert expressly stresses the difference between Baumgarten's definition of ontology for instance in $\S 4$ of Baumgarten'sMetaphysics and his own, for instance in $\S \S 3-13$ in the Architectonic. See Lambert (1787: 413, 114). Baumgarten, according to Lambert, searches for general predicates of being, while he, Lambert, aims at providing the first and simple grounds of cognition. Lambert might, however, have overlooked for instance $\S$ 5 of the Metaphysics, in which Baumgarten stresses the epistemological aspect of his ontology. 
an ontology that is essentially oriented towards experience: Based on simple concepts that are taken from experience and connected by means of practical postulates, it meets its ultimate demand, namely applyingits principles to experience again.

\section{Conclusion}

With this paper I hope to have shown that Lambert's discussion of Wolff's mathematical method is based on two criticisms which lead Lambert to a novel approach to metaphysics. Lambert tries on the one hand to revise Wolff's idea of fundamental concepts by providing what he takes to be a more profound theory of concepts. Instead of basing philosophy on general concepts, which are achieved by means of abstraction, Lambert bases his theory on simple concepts, which are taken directly from experience. On the other hand, Lambert introduces postulates. These practical instructions regulate the connection between simple concepts and account for their application to experience. Both transformations help Lambert to pursue Wolff's project of making metaphysics a science while avoiding at the same time the formality of Wolff's approach. This idea becomes especially apparent with regard to Lambert's notion of ontology. The aim of ontology, as Lambert sees it, is no longer to provide special metaphysics with its concepts. Rather, its ultimate criterion of success is that its principles are valid with regard to objects of experience. In this way, ontology becomes an independent science which constitutes one of those a priori sciences that Lambert takes to be exclusively oriented towards experience.

\section{Bibliography}

BAXMANN, Inge: Das Laokoon Paradigma. Zeichenregime im 18. Jahrhundert, Berlin, Akademie Verlag, 2000

BASSO, Paola: "Rien de mathématique dans la methodus mathematica wolfienne. La méthode «mathématique» de Wolff et les objections de Lambert" in: Lumiéres. Christian Wolff et la pensée encyclopédique européenne, Bordeaux, Presses Universitaires de Bordeaux, 2008,109-121.

BECK, Lewis W.: "Lambert und Hume in Kants Entwicklung von 1769 - 1772", Kant-Studien 60, 2, (1969), 123-130.

BIERBACH, Pier: Wissensrepräsentation - Gegenstände und Begriffe: Bedingungen des Antinomieproblems bei Frege und Chancen des Begriffssystems bei Lambert, Dissertation, 2001.

BLOMME, Henny: "La notion de 'système' chez Wolff, Lambert et Kant", Estudos Kantianos 3, 1, (2015), 105-126.

BRANDT, Reinhard: “The table of Judgment. Critique of Pure Reason A 67-76; B 92-101", North American Kant Society Studies in Philosophy 4, (1995), 1-147.

DUNLOP, Katherine: "Why Euclid's geometry brooked no doubt: J. H. Lambert on certainty and the existence of models", Synthese 167, 1, (2009), 33-65.

ENGFER, Hans-Jürgen: Philosophie als Analysis Studien zur Entwicklung philosophischer Analysiskonzeptionen unter dem Einfluss mathematischer Methodenmodelle im 17. und frühen 18. Jahrhundert, Stuttgart, Frommann-Holzboog, 1982.

HENRICH, Dieter: "Systemform und Abschlussgedanke - Methode und Metaphysik als Problem in Kants Denken", in: GERHARD, Volker (ed.): Kant und die Berliner Aufklärung, Boston, De Gruyter, 2001. 
HINTIKKA, Jaakko: "Kant on the Mathematical Method", The Monist 51, 3 (Kant Today: Part I), (1967), 352-375.

KAMBARTEL, Friedrich: "System und Begründung als wissenschaftliche und philosophische Ordnungsbegriffe bei und vor Kant" In: RITTER, J.; BLÜHDORN, J. (ed.): Philosophie und Rechtswissenschaft, Frankfurt am Main, Klostermann, (1969), 99-113.

LAMBERT, Johann H.: Über die Methode, die Metaphysik, Theologie und Moral richtieger zu beweisen, edited by K. Bopp, Berlin, Reuther und Reichard, 1918 [=Kantstudien, Ergänzungshefte 42]

LAMBERT, Johann H.: Neues Organon oder Gedanken über die Erforschung und Beziehung des Wahren und dessen Unterscheidung vom Irrthum und Schein, Teil I und II, Leipzig, 1764.

LAMBERT, Johann H.: Criterium Veritatis, edited by K. Bopp, Berlin, Reuther und Reichard, 1915. [=Kantstudien, Ergänzungshefte 36].

LAMBERT, Johann H.: Anlage zur Architektonik oder Theorie des Einfachen und Ersten in der philosophischen und mathematischen Erkenntniß, Riga, 1771.

LAMBERT, Johann H.: Fragment einer Systematologie. In: Texte zur Systematologie und zur Theorie der wissenschaftlichen Erkenntnis, edited by G. Siegwart, Hamburg, Meiner, 1988.

LAMBERT, Johann H.: Die Theorie der Parallellinien. Magazin für die reine und angewandte Mathematik, edited by J. Bernoulli, Berlin, Müller, Täubel, 1786.

LAMBERT, Johann H.: Logische und philosophische Abhandlungen, Teil II, Berlin, Bernoulli, 1782. Reprint: Hildesheim 1969. XII.

LAMBERT, Johann H.: Lamberts deutscher gelehrter Briefwechsel, Berlin/Leipzig, Bernoulli, 1782.

LAYWINE, Alison: "Kant and Lambert on geometrical postulates in the reform of metaphysics", In: FRIEDMAN, M.; DOMSKI, M. (ed.), Discourse on a New Method: Reinvigorating the Marriage of History and Philosophy of Science, Chicago, Open Court, 2010.

MANCHESTER, Paula: "Kant's Conception of Architectonic in its Historical Context", Journal of the History of Philosophy 41, 2, (2003), 187-207.

RIVERO, Gabriel: Zur Bedeutung des Begriffs Ontologie bei Kant, Berlin, De Gruyter, 2014.

ROS, Arno: “„Bedeutung“, „Idee“ und „Begriff”: Zur Behandlung einiger bedeutungstheoretischer Paradoxien durch Leibniz”, Studia Leibnitiana 21, 2, (1989), 133-154.

SIEGWART, Geo: Texte zur Systematologie und zur Theorie der wissenschaftlichen Erkenntnis, Hamburg, Meiner, 1988.

VOLLRATH, Ernst: "Die Gliederung der Metaphysik in eine metaphysica generalis und eine metaphysica specialis", Zeitschrift für philosophische Forschung 16, 2, (1962), 258-284.

WAIBEL, Violetta: "Die Systemkonzeptionen bei Lambert und Wolff” in: STOLZENBERG, J. (ed.): Christian Wolff und die europäische Aufklärung, Teil 2, Hildesheim, Georg Olms, 2007.

WELLMANN, Gesa: "Lamberts Begriff eines metaphysischen Systems", Archiv für Begriffsgeschichte 58, (2017), in press.

WOLFF, Christian: Vernünftige Gedanken von den Kräften des menschlichen Verstandes und ihrem richtigen Gebrauche in Erkenntnis der Wahrheit, Hildesheim, Georg Olms, 1713/1987.

WOLFF, Christian: Ausführliche Nachricht von seinen eigenen Schriften, Frankfurt am Main, Andredische Buchhandlung, 1733. 
WOLFF, Christian: "De differentia intellectussystematici\& non-systematici". Translated, introduced and editied by M. Albrecht, Aufklärung. Interdisziplinäre Jahrbuch zur Erforschung des 18. Jahrunderts und seiner Wirkungsgeschichte 23, (1729/2011), 229-302.

WOLTERS, Gereon: Basis und Deduktion. Studien zur Entstehung und Bedeutung der Theorie der axiomatischen Methode bei J. H. Lambert (1728-1777), Berlin, de Gruyter, 1979.

ZÖLLER, Günter: ““'Die Seele des Systems”: Systembegriff und Begriffssystem in Kants Transzendentalphilosophie", in: FULDA, H. F.; STOLZENBERG, J. (eds.): Architektonik und System in der Philosophie Kants, Hamburg, Meiner, 2001, 54-72. 


\title{
Acerca del alcance objetivo de las prescripciones metodológicas de la función regulativa de la razón teórica en la Crítica de la razón pura de Kant
}

\author{
MARTÍN ARIAS AlBisU ${ }^{1}$
}

\begin{abstract}
Resumen
En el "Apéndice a la dialéctica trascendental" de su Crítica de la razón pura, Kant presenta prescripciones metodológicas para el conocimiento empírico. Expondré los dos conjuntos de prescripciones más importantes de esa metodología: a) la necesidad de introducir conceptos "teóricos"; b) el uso hipotético de la razón y la exigencia de sistematicidad, que se refieren a leyes empíricas y a los conceptos vinculados con tales leyes. Cada uno de estos conjuntos de prescripciones tiene una dimensión objetiva propia, esto es, su aplicación es condición de posibilidad de la formación y validez objetiva de determinadas clases de leyes y/o conceptos. Mi objetivo es presentar las diferencias y relaciones mutuas entre esas diferentes dimensiones objetivas.
\end{abstract}

Palabras clave: Crítica de la razón pura, ciencia empírica, regulativo, metodología, objetividad

\section{On the Objective Scope of the Methodological Prescriptions of the Regulative Function of Theoretical Reason in Kant's Critique of Pure Reason}

\begin{abstract}
In the "Appendix to the Transcendental Dialectic" of his Critique of Pure Reason, Kant presents methodological prescriptions for empirical knowledge. I will expound the two most important sets of prescriptions of this methodology: a) the necessity of introducing "theoretical" concepts; b) the hypothetical use of reason and the demand for systematicity, which refer to empirical laws and to the concepts related to such laws. Each of these sets of prescriptions has its own objective dimension, that is, its application is a condition of the possibility of the formation and objective validity of determinate kinds of laws and/or concepts. My aim is to present the differences and mutual relations between these different objective dimensions.

Keywords: Critique of Pure Reason, empirical science, regulative, methodology, objectivity
\end{abstract}

Introducción

En el extenso cuerpo de la "Dialéctica trascendental" de su Crítica de la razón pura (KrV, 1781/1787), Kant intentó justificar la tesis según la cual las ideas de la razón teórica no pueden brindar conocimiento de los objetos suprasensibles alma, mundo y Dios. Al final de esa división de la Crítica, Kant introdujo una concisa sección denominada "Apéndice a la dialéctica trascendental" ("Anhang zur transscendentalen Dialektik" = AtD). Esta sección contiene dos partes, a saber, "Del uso regulativo de las ideas de la razón pura" (AtD1) y "Del propósito último de la dialéctica natural

\footnotetext{
${ }^{1}$ Universidad Nacional del Rosario - CONICET
} 
de la razón humana" (AtD2). En ellas se expone el legítimo uso regulativo de las ideas de la razón teórica con respeto al conocimiento empírico del entendimiento. ${ }^{2}$

Varios comentaristas señalaron que en el texto del AtD hay una contradicción o, al menos, cierta tensión, entre una concepción metodológica o subjetiva de la función regulativa de la razón teórica y otra transcendental u objetiva. ${ }^{3}$ A diferencia de tales comentaristas, estableceré una clara demarcación entre las concepciones subjetiva y objetiva de la función regulativa de la razón teórica que permitirá mostrar que ellas no son contradictorias. La novedad de mi contribución consiste en que, a fin de determinar la diferencia y la articulación entre las dos mencionadas concepciones, tendré en cuenta las peculiaridades de las prescripciones metodológicas exigidas por la función regulativa de la razón teórica que considero más importantes, a saber: i) la necesidad de introducir ciertas ideas de la razón que denomino 'conceptos teóricos' (como el de 'flogisto', que se utilizaba en la química flogística de la época de Kant); ii) el uso hipotético de la razón con sus cinco criterios para la admisión y/o evaluación de las hipótesis de leyes empíricas que emplean conceptos teóricos y empíricos (aquí examinaré, fundamentalmente, las que emplean al menos un concepto teórico); y iii) la exigencia de máxima unidad y extensión sistemáticas para esa clase de hipótesis y esas clases de conceptos.

Las prescripciones ii) y iii) están íntimamente vinculadas. Efectivamente, la exigencia de sistematicidad planteada por la prescripción iii), en la medida en que se ejerce sobre hipótesis de leyes empíricas, puede considerarse como un criterio para la evaluación de estas hipótesis, el cual se agrega a los cinco criterios de esa clase que, de acuerdo con la prescripción ii), deben aplicarse necesariamente. De hecho, como se verá, la posibilidad de integrar una hipótesis de ley empírica en un sistema de esas leyes es uno de los criterios que se deben emplear para evaluar el valor de verdad de esa hipótesis. ${ }^{4}$ Por tal motivo, las prescripciones ii) y iii) serán analizadas aquí conjuntamente.

El estudio de esta problemática me permitirá sostener la tesis según la cual las prescripciones metodológicas i) y ii)-iii) determinan concepciones objetivas de la función regulativa de la razón teórica como correlatos de cada una de ellas que son, al menos parcialmente, diferentes, aunque existen conexiones y articulaciones entre las mismas. ${ }^{5}$ Por 'correlato objetivo' o 'dimensión objetiva' de una prescripción metodológica entiendo aquí la(s) clase(s) determinada(s) de leyes y/o conceptos cuya formación y validez objetiva tienen como condición de su posibilidad la aplicación de esa prescripción. A su vez, como intentaré mostrar, tales leyes y conceptos son condiciones de posibilidad de ciertos tipos de experiencia.

A continuación examinaré las prescripciones i) y ii)-iii) en ese orden y en dos secciones diferentes.

\footnotetext{
${ }^{2}$ A fin de simplificar, me referiré al uso regulativo de las ideas de la razón teórica con la expresión 'función regulativa de la razón teórica'.

${ }^{3}$ Acerca de la tensión o contradicción entre una concepción subjetiva y otra objetiva de la función regulativa de la razón teórica, cf. Kemp Smith 1923/2003: 543-561; Bennett 1974: 275 ss.; Kitcher 1986: 207. Por otro lado, los textos de Kant se citan a partir de la Akademie-Ausgabe y según las siglas establecidas en el sitio web de la Kant-Forschungsstelle de la Universidad de Mainz (http://www.kant.uni-mainz.de/ks/abhandlungen.html). La primera Crítica $(\mathrm{KrV})$ es citada, como es habitual, de acuerdo con la paginación de la primera $(\mathrm{A}=1781)$ y segunda $(\mathrm{B}=1787)$ ediciones. Véase la bibliografía para más detalles y una indicación de las traducciones castellanas utilizadas. Modifiqué algunos pasajes de esas traducciones para ofrecer versiones más fieles al original alemán.

${ }^{4}$ Cf. infra, n. 9 y Sección 2. Por otro lado, una argumentación presentada en un artículo de Mario Caimi permite mostrar la complementariedad de las dimensiones subjetiva y objetiva de la exigencia regulativa de sistematicidad referida fundamentalmente a los conceptos empíricos (cf. Caimi 1995: 311-317 y Sección 2).

${ }^{5}$ Acerca de estas prescripciones y las relaciones entre ellas, véase Arias Albisu 2015. Por otro lado, el principio trascendental de la facultad de juzgar reflexionante de la Crítica de la facultad de juzgar (KU) no puede examinarse en este artículo por razones de espacio.
} 
1. La exigencia regulativa de introducción de conceptos teóricos y su dimensión objetiva

La exigencia regulativa de introducir las ideas de la razón que aquí denomino 'conceptos teóricos' es presentada por Kant en el quinto párrafo del AtD1 (A645-646/B673-674). ${ }^{6}$ Efectivamente, en ese párrafo Kant presenta ideas de la razón como las de tierra pura y de sales. Esas ideas no tienen, en sentido estricto, objetos que les correspondan adecuadamente en la naturaleza, dado que se originan en la razón teórica. Sin embargo, es necesario introducir ideas como las mencionadas para "determinar debidamente la participación que cada una de esas causas naturales [MA: tierra pura, etc.] tiene en el fenómeno". De esta manera, "todas las materias se reducen a tierras (por así decir, el mero peso), a sales y cuerpos combustibles (que vienen a ser la fuerza), y finalmente a agua y aire, como vehículos (por así decir, como máquinas por medio de las cuales las precedentes actúan)". Se procuran explicar así, mecánicamente, los efectos químicos recíprocos de esas materias.

Los ejemplos de conceptos teóricos ofrecidos por Kant pertenecen a la química flogística de la época. De hecho, la expresión 'cuerpos combustibles' [brennliche Wesen] hace referencia al principio de la inflamabilidad o "flogisto". A fin de explicar las concisas afirmaciones de Kant, expondré primeramente, en general, la noción de concepto de teórico, y mostraré luego más detalladamente la vinculación entre estos conceptos teóricos y la química.

Para Kant, los conceptos teóricos se originan, en lo que a "la completa pureza" se refiere, en la razón teórica ( $\mathrm{KrV}, \mathrm{A} 646 / \mathrm{B} 674)$. El entendimiento emplea sus categorías y conceptos empíricos para hacer posible, por ejemplo, una experiencia de la calcinación de un trozo de plomo. Sin embargo, a fin de explicar este proceso es necesario que la razón realice una determinada labor conceptual. Semejante labor consiste en interpretar procesos como el mencionado como el resultado de la operación de ciertos elementos puros contenidos en objetos empíricos. De esta manera, se intenta mostrar que las propiedades y procesos químicos de esos objetos dependen de la presencia y la operación de esos elementos. Tales elementos son representados por la razón de manera pura, esto es, sin tener en cuenta los otros elementos con los cuales ellos se encuentran siempre mezclados en la naturaleza. Por el contrario, el entendimiento sólo puede representar objetos empíricos en los cuales tales elementos están mezclados unos con otros. Denominaré a los conceptos que son representaciones de estos elementos puros, como adelanté, 'conceptos teóricos', y a esos elementos puros mismos, 'objetos teóricos'?

Los objetos teóricos son postulados en hipótesis de leyes empíricas científicas que incluyen en su formulación los conceptos teóricos que se refieren a los primeros. Tales objetos teóricos no son ni objetos empíricos ni construcciones de conceptos matemáticos en la intuición pura. Más bien, los objetos teóricos son entidades puramente ideales. Ellos no están sometidos a todas las variaciones que sufren los objetos empíricos en virtud de estar compuestos por mezclas de diferentes elementos puros. De hecho, los objetos teóricos sólo pueden variar de determinadas maneras, las cuales son previstas en la formulación de la hipótesis que se refiere a ellos. Además, como los objetos teóricos no son postulados para explicar un fenómeno singular, sino una(s) clase(s) determinada(s) de fenómenos, estos objetos hacen posible una explicación no sólo precisa, sino también económica, de esa(s) clase(s) de fenómenos. Aunque los fenómenos que, según la hipótesis, contienen como elemento puro

${ }^{6}$ Anteriormente, esto es, al comienzo del AtD1, Kant expone de manera preliminar una concepción de las ideas de la razón teórica relacionada con la exigencia de sistematicidad del conocimiento empírico (cf. KrV, A642-645/B670-673). Esta concepción se aplica fundamentalmente a dos diferentes clases de estas ideas, a saber, las ideas como principios (expuestas más adelante en AtD1) y como entes de razón (presentadas en AtD2). La exigencia regulativa de sistematicidad será examinada en la Sección 2.

7 La designación "concepto teórico" es empleada por Buchdahl (1965, 1969 y 1992), Wartenberg (1979 y 1992) y Brittan (1992). Buchdahl también emplea las designaciones "concepción teórica" (1965) y "constructo teórico" (1967). En cambio, Rauscher hace referencia a "ideas mundanas" (2010). Aquí sigo principalmente a Brittan, quien también emplea el concepto de "objeto teórico" en un sentido similar al que aquí le concedo. Por otro lado, Carrier (2001) y McNulty (2015) estudian el empleo de las ideas de la razón que denomino "conceptos teóricos" en el ámbito de la química de la época. 
el objeto teórico postulado por ella pueden ser diferentes, ese objeto teórico pensado como contenido en ellos es por definición siempre el mismo. Por tal motivo, un descubrimiento acerca de un objeto teórico es válido para todos los fenómenos en los que se piensa que ese objeto está contenido, así como para todos los fenómenos vinculados por la hipótesis en cuestión con los primeros. ${ }^{8}$

En su Crítica de la razón pura, Kant se ocupa del problema de la validez objetiva de ciertos tipos de conceptos - principalmente las categorías - que no son sino los conceptos empíricos y los matemáticos. Aquí es de interés presentar solamente una interpretación del sentido en el que los conceptos teóricos pueden adquirir cierta validez objetiva. ${ }^{9}$ Como estos conceptos se refieren a elementos puros que en la naturaleza se encuentran únicamente mezclados con otros, no es posible que a esos conceptos les correspondan intuiciones enteramente adecuadas. Sin embargo, justamente el hecho de que los elementos puros representados por conceptos teóricos sean entendidos, por definición, como contenidos en objetos empíricos, puede considerarse como un criterio de una cierta validez objetiva de tales conceptos. Pues, de esta manera, los conceptos teóricos pueden referirse efectivamente a intuiciones empíricas, aunque ellas no sean enteramente adecuadas como, por ejemplo, en el caso de los conceptos empíricos. Por otro lado, ya se señaló que los objetos teóricos son postulados en hipótesis de leyes empíricas científicas que emplean conceptos teóricos y procuran explicar los fenómenos dados. Por tanto, estimo que esta validez objetiva de los conceptos teóricos también depende de que las hipótesis que los emplean sean puestas a prueba exitosamente mediante experimentos. $^{10}$

A continuación indicaré cuál es el tipo de ciencia que, según Kant, requiere el empleo de conceptos teóricos. Como adelanté en el pasaje mencionado al principio de esta Sección, en el que se introduce la noción de concepto teórico, la ciencia considerada es la química (A645-646/B673-674). Kant presenta en AtD1 otros ejemplos de ejercicio de la función regulativa de la razón teórica en el ámbito de esa disciplina. ${ }^{11}$ Friedman ha mostrado que durante el período crítico Kant adhería a la química flogística tradicional desarrollada especialmente por Georg E. Stahl (1659-1734). ${ }^{12}$ En un conocido pasaje del "Prólogo a la segunda edición" de la primera Crítica (BXII-XIV), Kant vincula los experimentos de, entre otros, Stahl, con una "revolución del modo de pensar" que puso a la ciencia de la naturaleza en la carretera de la genuina ciencia. ${ }^{13}$ Kant indica que tiene en cuenta la ciencia de la naturaleza únicamente en tanto que está basada en principios empíricos. Según el nuevo modo de pensar, en la ciencia de la naturaleza así entendida la razón debe dirigirse a la naturaleza con principios y experimentos diseñados de acuerdo a estos. De este modo, la razón "es instruida por ésta [MA: la naturaleza], pero no en calidad de un escolar que deja que el maestro le diga cuanto quiera, sino [en calidad] de un juez en ejercicio de su cargo, que obliga a los testigos a responder a las preguntas que él les plantea" (BXIII). La oposición entre la escucha atenta de lo que dice el maestro y el planteamiento de la obligación de responder a ciertas preguntas por parte del juez hace referencia, como está claro en el contexto, a la oposición entre 'observaciones contingentes' hechas sin ningún plan previo y la efectuación de experimentos de acuerdo con un cierto planeamiento. Puede

\footnotetext{
${ }^{8}$ Brittan expresa una tesis similar cuando afirma que "los objetos teóricos son como los matemáticos, enteramente representativos de la clase a la que pertenecen" (Brittan 1992: 176).

${ }^{9}$ Los conceptos tienen validez objetiva cuando, a grandes rasgos, tienen intuiciones empíricas que les corresponden. Cf. $\mathrm{KrV}$, A238-246/B297-302

${ }^{10}$ Véanse al comienzo de esta Sección las tesis de Kant sobre la necesidad de determinar la participación de cada 'causa natural' (concepto u objeto teórico) en el fenómeno, y de explicar las interacciones químicas de las materias (en infra, n. 13 presento un experimento mencionado por Kant). En la Sección 2 mostraré que las hipótesis que emplean conceptos teóricos pueden tornarse más probables cuando son puestas a prueba empíricamente con éxito, pero nunca pueden alcanzar una necesidad estricta, sino sólo más probabiativa.

${ }^{11}$ Cf. KvR, A652-653/B680-681 y A657/B685. Cf. Sección 2.

${ }^{12}$ Friedman 1992: 240-242 y 264-267. Cf. infra, n. 17.

${ }^{13}$ También se mencionan experimentos de Galileo y Torricelli. Cf. infra, n. 17.
} 
concluirse que la clase de disciplina que requiere conceptos teóricos es, al menos, la química experimental de Stahl. ${ }^{14}$

Un análisis de algunos pasajes del "Prólogo" a los Primeros principios metafísicos de la ciencia de la naturaleza (MAN, 1786), permitirá profundizar la comprensión del problema del tipo de ciencia que emplea conceptos teóricos. Allí se afirma que la "doctrina de la naturaleza" se divide en "ciencia de la naturaleza" y en "doctrina histórica de la naturaleza". Esta última contiene "hechos sistemáticamente ordenados de las cosas de la naturaleza" y se divide a su vez en "descripción de la naturaleza" e "historia de la naturaleza" (MAN, AA 04: 468). ${ }^{15}$

La ciencia de la naturaleza, que es la que aquí nos interesa, puede ser ciencia o bien de un modo propio o bien de uno impropio. La ciencia de la naturaleza en sentido propio del término, esto es, la física matemática, contiene una parte pura. Esta parte pura está compuesta por leyes de la naturaleza que son de carácter a priori y alcanzan entonces certeza apodíctica. Tal parte pura consta de una parte trascendental y una parte metafísica. La parte trascendental contiene las leyes a priori que determinan la forma de una naturaleza en general. Estas leyes son los principios del entendimiento puro expuestos en la primera Crítica (Krv, A148/B187 ss.). La parte metafísica es una metafísica de la naturaleza corpórea y consiste en el conocimiento a priori acerca de la totalidad de los objetos corpóreos que puede alcanzarse introduciendo un concepto empírico muy general de materia. Esta metafísica es presentada en MAN y fundamenta la aplicación adecuada de la matemática a los fenómenos del sentido externo (cf. MAN, AA 04: 468-473).

La ciencia de la naturaleza en sentido impropio, esto es, al menos, la química, contiene únicamente leyes basadas en la experiencia (MAN, AA 04: 468). La química es una ciencia en sentido impropio porque no posee una parte pura y la matemática no puede aplicarse adecuadamente dentro de su ámbito. ${ }^{16}$ Por otro lado, considero que la química es una ciencia, aunque en un sentido impropio, porque puede ser "arte sistemático o doctrina experimental" (MAN, AA 04: 471). En primer lugar, Kant conecta la sistematicidad con la cientificidad en la $\mathrm{KrV}$ (832/B860 ss.) y en MAN (AA, 04: 467). ${ }^{17}$ En segundo lugar, la conexión entre cientificidad y carácter experimental en, por ejemplo, la química de Stahl, se indica en el pasaje del "Prólogo a la segunda edición" de KrV (BXIIXIV) y que comenté anteriormente. Además, al comienzo de esta Sección se expuso una conexión entre experimentación y empleo de conceptos teóricos en el ámbito de esa química. Las leyes empíricas científicas que emplean conceptos teóricos pertenecientes a la química de Stahl, tal como el de flogisto, se deben poner a prueba mediante experimentos. Estimo, entonces, que los conceptos teóricos se emplean en la ciencia en sentido impropio del término, y que este empleo, junto con la aplicación de la exigencia regulativa de sistematicidad, explican el carácter científico de este tipo de

\footnotetext{
${ }^{14}$ En el mencionado pasaje del "Prólogo a la segunda edición" de su Crítica de la razón pura, Kant alude a un experimento realizado por Stahl. Kant afirma que el químico alemán "transformó metales en cal y ésta otra vez en metal, quitándoles algo y dándoselo de nuevo" (BXII-XIII). Stahl en primer lugar calcinó plomo para producir cal o ceniza de plomo (la cal o ceniza de un metal era concebida como un tipo de tierra, y hacía referencia a lo que hoy se denomina "óxido" de un metal). En este proceso, aparentemente, el flogisto contenido en el plomo fue removido. Posteriormente, Stahl quemó carbón en presencia de la cal de plomo y descubrió que esta última se transformó en un trozo metálico de plomo con sus características distintivas. Tal proceso se explicaría por la liberación del flogisto contenido en el carbón y su absorción por parte de la cal de plomo. Este experimento permitiría concluir que todas las calcinaciones y combustiones se explican por el mismo mecanismo de liberación del flogisto, y que el flogisto es el mismo en todos los reinos de la naturaleza, por ejemplo en el mineral (plomo) y en el vegetal (carbón). En otras palabras, este experimento sustentaría la hipótesis siguiente: el flogisto es el principio de la inflamabilidad. Es claro que, según Kant, lo que Stahl "quitó" y "dio de nuevo" es el objeto teórico "flogisto". Sigo las exposiciones de este experimento ofrecidas por Carrier y McNulty (cf. Carrier (2001: 217-218) y McNulty (2015: 5-6). Cf. también Stahl (1718: 119-120).

${ }^{15}$ Para un tratamiento de la diferencia entre "historia de la naturaleza" y "descripción de la naturaleza" en la evolución del pensamiento kantiano y en otros pensadores contemporáneos y posteriores, cf. Sloan (2006).

${ }^{16}$ En el "Prólogo" a MAN se afirma que el carácter científico en sentido propio que posee la física matemática depende de su parte pura (AA 04: 469 ss.) y de la aplicabilidad de la matemática en su ámbito (AA 04: 470-471).

${ }^{17}$ También la física matemática es sistemática. Sin embargo, el problema de en qué sentido este saber es sistemático no será tratado aquí, porque me ocupo de la sistematicidad sólo en la medida en que afecta al tipo de disciplina que contiene leyes empíricas científicas que emplean conceptos teóricos.
} 
ciencia. ${ }^{18}$ Las exigencias regulativas de introducción de conceptos teóricos y sistematización del conocimiento empírico están vinculadas entre sí, porque, por ejemplo, las leyes que emplean conceptos teóricos, a diferencia de las generalizaciones inductivas, son precisas y económicas y pueden ser integradas en sistemas estrictamente coherentes. ${ }^{19}$ Volveré a tratar en la Sección 2 el problema de las relaciones entre las dos exigencias mencionadas. ${ }^{20}$ Por otro lado, el examen del problema del tipo de ciencia tenido en cuenta en el AtD llevado a cabo hasta el momento nos permite formular dos conclusiones. En primer lugar, esta clase de disciplina esuna ciencia en un sentido impropio del término. A diferencia de la física matemática, la ciencia en sentido impropio es ciencia únicamente en virtud de su carácter sistemático y experimental. En segundo lugar, estetipo de ciencia incluye, al menos, a la química. ${ }^{21}$ Adelanto que, por motivos de espacio, no puede llevarse acabo aquí el extenso análisis de textos que requiere la consideración del problema de si, según el pensamiento kantiano en su período crítico, esa clase de ciencia está integrada únicamente por la química o incluye también otras disciplinas.

Se expusieron las características de los conceptos teóricos, su vinculación con las leyes empíricas científicas y la clase de ciencia que requiere leyes de este tipo. De acuerdo con los pasajes

${ }^{18}$ Según Friedman, MAN muestra cómo el ámbito de lo propiamente empírico es fundamentado determinadamente en el concepto trascendental de una naturaleza en general. La aplicación de los principios trascendentales del entendimiento al concepto empírico de materia consigue fundamentar la ley empírica newtoniana de gravitación universal (Friedman, 1992: 263). No dispongo aquí de espacio para evaluar esta interpretación. (Véanse las lecturas diferentes de Buchdahl 1965: 202 y 207-208, 1971: 34-44; Butts 1986: 190 ss. y Wartenberg 1979: 412-413, 1992: 240-241. Kant presenta en AtD1 el ejemplo de sistematización de las leyes del movimiento de los cuerpos celestes, y alude brevemente allí a la fuerza gravitacional como causa de ellas. Cf. KvR, A662 663/B690-691. Sin embargo, no estoy al tanto de base textual suficiente para valorar el señalamiento de Buchdahl y Butts de que la fuerza gravitacional es un concepto teórico del mismo orden que los demás. Cf. Buchdahl 1967: 217, 1992: 258 y $264-265$ y Butts, 1986: 190 ss.). De acuerdo con Friedman, ciencias no matemáticas como la química de Stahl y la totalidad de la parte puramente experimental de la fisica no son comprendidas por la fundamentación de MAN (Friedman 1992: 174 ss., 240-242, 250 ss., 263-264 y 266-267). Asimismo, Friedman indica que, en el siglo XVIII, esas disciplinas pertenecientes a la "física experimental" se distinguían de las pertenecientes a la "física matemática", a saber, la mecánica racional, la óptica, la astronomía y la teoría newtoniana de la gravitación universal (Friedman 1992: 239, n. 38). Por último, Friedman estima que una de las motivaciones de Opus Postumum $(O P)$ es proveer una fundamentación para disciplinas incipientes del primer tipo como las ciencias del calor, la luz, la electricidad y el magnetismo, así como la química de Lavoisier (Friedman 1992: 263-264. Friedman observa que Kant habría adoptado la química de Lavoisier a más tardar en 1795. Ibíd.: 289). Como indicaré más adelante, no hay espacio aquí para examinar el problema de si en $\mathrm{KrV}$ o MAN Kant considera la química aisladamente o como perteneciente al conjunto de disciplinas que Friedman denomina "física experimental".

${ }^{19}$ Las generalizaciones de los fenómenos sustentadas en la observación casual efectuada hasta un cierto momento podrían denominarse leyes (en sentido amplio) empíricas ordinarias. Una de estas generalizaciones inductivas es el enunciado "el frío intenso congela el agua". Estos enunciados no son leyes en sentido estricto porque de ninguna manera pueden tenerse por necesarios. La experiencia ordinaria no implica necesariamente ni una coherencia ni una conexión mutua entre las diversas generalizaciones inductivas. Por tanto, esas generalizaciones no pueden ser integradas en sistemas estrictamente coherentes. Acerca de la necesidad "regulativa" de las leyes empíricas científicas, cf. infra, n. 19 y n. 46.

${ }^{20}$ Más arriba mostré cómo los conceptos y objetos teóricos hacen posibles explicaciones económicas y precisas de ciertas clases de fenómenos. En virtud de esta circunstancia, Brittan sostiene que las leyes que se refieren a objetos teóricos pueden ser tenidas por necesarias, al menos en tanto hayan sido verificadas (Brittan 1992: 176-177). Coincido parcialmente con Brittan, porque, como acabo de señalar, estimo que esas leyes también deben poder ser integradas en un sistema a fin de poder adquirir una necesidad que se podría llamar "regulativa" (cf. infra, n. 46). Para Brittan, en cambio, la sistematicidad es solamente una garantía de que nuestras teorías se refieren a un mundo único (Brittan 1992: 177-178).

${ }^{21}$ Como ya mencioné, en AtD1 Kant también hace referencia en una ocasión a las leyes del movimiento de los cuerpos celestes (cf. supra, n. 17). En lo que hace a la doctrina empírica del alma o psicología empírica, Kant afirma en MAN que ella no puede ser una ciencia, sino sólo una "descripción natural del alma" perteneciente a la doctrina histórica de la naturaleza. En primer lugar, esta psicología no es una ciencia en sentido propio porque la matemática no puede aplicarse adecuadamente a los fenómenos del sentido interno (acerca del sentido en el que la matemática no es aplicable de manera adecuada ni a la química ni a la psicología, cf. Nayak y Sotnak 1995). En segundo lugar, en la psicología empírica, a diferencia de la química, no es posible la experimentación ni la observación rigurosa: los fenómenos observados no pueden mantenerse separados ni ser recombinados a voluntad, no es posible someter a otro sujeto pensante a experimentos y la observación misma altera el estado de lo observado. Por tanto, esta psicología tampoco es una ciencia en sentido impropio como la química. Ciertamente, esa psicología puede aspirar a una cierta sistematicidad (MAN, AA 04: 471). Sin embargo, esta sistematicidad es puramente descriptiva y no conduce entonces a la formación de sistemas jerárquicos explicativos de leyes empíricas científicas. Estas leyes —esto es, las que emplean conceptos teóricos - deben ser puestas a prueba mediante experimentos, y no hay lugar para ellos en el ámbito de la psicología empírica. Por otro lado, en AtD1 Kant afirma que se debe intentar reducir las fuerzas de la mente (sensación, imaginación, memoria, etc.) a una única fuerza fundamental (A648-649/B676-677). En caso de que esta fuerza fundamental sea un concepto teórico, éste sería de una clase diferente de los utilizados en la química, puesto que, según lo dicho, no sería posible establecer una cierta validez objetiva del mismo mediante experimentación ni observación rigurosa. Sin embargo, estimo que la concepción kantiana de esa psicología en el AtD se revela cuando, en AtD2, se introduce la función regulativa de la idea del alma en tanto ente de razón. En virtud de esta función regulativa es posible una sistematización descriptiva de los fenómenos internos (KrV, A672/B700 y A682-684/B710-712). Acerca de las mencionadas tesis del AtD, cf. Sección 2 
citados y comentados, la doctrina kantiana de los conceptos teóricos podría interpretarse como una metodología del conocimiento empírico científico o una metateoría de la ciencia empírica fundada en una mera conveniencia subjetiva. Sin embargo, en un pasaje del AtD1 en el que se exponen los tres principios o leyes sistematizadores de la razón que presentaré en la Sección 2, Kant hace observaciones que considero válidas para la función regulativa de la razón teórica en general. Las leyes mencionadas juzgan que la "economía de las causas fundamentales" y otros requerimientos sistematizadores del conocimiento empírico "son [todos] en sí mismos conformes a la razón y adecuados a la naturaleza, y que por consiguiente esos principios llevan consigo su propia [carta de] recomendación de manera directa, y no meramente como procedimientos del método" (KrV, A660661/B688-689). ${ }^{22}$ Más aún, Kant señala que esos tres principios 'lógicos' son fundamentados por principios 'trascendentales'. ${ }^{23}$

Ahora ofreceré una interpretación de esa pretensión de objetividad o adecuación a la naturaleza, e incluso de carácter trascendental, de la función regulativa de la razón teórica en el caso de su exigencia de introducir conceptos teóricos. Como se indicó anteriormente, los conceptos teóricos pueden adquirir cierta validez objetiva cuando, en primer lugar, los elementos puros u objetos teóricos que ellos representan son pensados como contenidos en objetos empíricos $\mathrm{y}$, en segundo lugar, las hipótesis de leyes que los emplean son puestas a prueba empíricamente con éxito. Además, puede interpretarse que estos conceptos son 'transcendentales' en el sentido, por cierto amplio, de que son condiciones de posibilidad, no ya de la experiencia en general, sino de lo que se podría llamar una 'experiencia científica'. ${ }^{24}$

Al considerar la vinculación entre conceptos teóricos y objetos empíricos, se desprende además que los conceptos teóricos que se refieren a objetos teóricos pensados como contenidos en cierta(s) clase(s) de objetos empíricos pueden ser incluidos como predicados o, según la terminología kantiana, "conceptos parciales" o "notas discursivas", en el contenido lógico del (de los) concepto(s) empírico(s) de esa(s) clase(s) de objetos empíricos. ${ }^{25}$ Podría pensarse, por ejemplo, que en los conceptos empíricos de nuestra experiencia externa ordinaria de objetos materiales están incluidos como conceptos parciales, si bien obscuramente, conceptos teóricos pertenecientes a una teoría simplificada de los elementos de la materia.

Cabe señalar que los conceptos empíricos, si bien no son condiciones de posibilidad de la experiencia de objetos empíricos en general, son por cierto condiciones de posibilidad de la experiencia de objetos empíricos en tanto objetos empíricos de ciertas clases. Por tanto, los conceptos teóricos, en la medida en que estén incluidos en los contenidos lógicos de los conceptos empíricos, pueden desempeñar el papel de condiciones de posibilidad de la experiencia en tanto que ella requiere conceptos empíricos, y ser entonces 'transcendentales' en un sentido amplio. ${ }^{26}$ Tal vez únicamente en este sentido tenga la exigencia regulativa de introducir conceptos teóricos una dimensión objetiva con respecto a la experiencia ordinaria. Sin embargo, tal exigencia regulativa no posee necesariamente esa

\footnotetext{
${ }^{22}$ La sistematización efectuada por la razón teórica no "es una operación meramente económica" de esa facultad. Tampoco es un "ensayo hipotético" fundamentado únicamente por su rendimiento de unidad en la explicación (KrV, A653/B681).

${ }^{23} \mathrm{Cf} . \mathrm{KrV}, \mathrm{A} 650-651 / \mathrm{B} 678-679$, A653-654/B681-682, A656-657/B684-685 y A660-661/B688-689.

${ }^{24}$ Hasta donde sé, Kant no usa la expresión "experiencia científica". Cf. infra, n. 26.

${ }^{25}$ El contenido lógico de un concepto es lo que se piensa en el mismo y está integrado por un concepto parcial o nota discursiva, o un conjunto de ellos. Los conceptos parciales o notas discursivas son representaciones universales y discursivas de propiedades que comparten todos los objetos que son casos particulares del concepto en cuestión. Ejemplos de notas discursivas contenidas en el concepto de cuerpo son la extensión, la impenetrabilidad y la figura $(\mathrm{KrV}, \mathrm{B} 12$. Sobre estas nociones, cf. Log, AA 09: 58 ss., 91 ss.).

Si bien hay un pasaje en el que Kant distingue las notas discursivas de los conceptos parciales (Log, AA 09: 95), estoy de acuerdo con Paton cuando mantiene que, para simplificar, es indicado desatender ese pasaje (Paton 1936/1970: I, 195-196). Kant mismo ignora la distinción mencionada en otros pasajes (cf. A43/B60 y Log, AA 09: 59-62).

${ }^{26} \mathrm{Mi}$ tesis es que la experiencia ordinaria puede envolver, y generalmente envuelve, el empleo de conceptos teóricos, aunque no necesariamente. Me opongo así, en primer lugar, a Brittan, quien sostiene que la experiencia ordinaria requiere conceptos teóricos para ser objetiva (Brittan 1992: 177). Además, mi tesis es diferente de la de Buchdahl, quien sostiene que, para Kant, el lenguaje observacional y el lenguaje teórico llevan vidas relativamente independientes (Buchdahl 1967: 222).
} 
dimensión objetiva, porque es perfectamente posible una experiencia ordinaria sin la intervención de conceptos teóricos. ${ }^{27}$

En síntesis, la exigencia regulativa de introducir conceptos teóricos tiene una dimensión objetiva y trascendental con respecto a una experiencia científica, y puede tener una dimensión objetiva y trascendental con respecto a la experiencia ordinaria. En lo que hace a este último punto, coincido parcialmente con una tesis de Peter Krausser. Aunque este último estima que su tesis es válida para la exigencia regulativa de sistematicidad, la presentaré aquí con referencia a la exigencia regulativa vinculada con los conceptos teóricos. Según Krausser, la función regulativa de la razón teórica puede entenderse como una versión temprana de la concepción, corriente hoy en día, de la carga teórica de la experiencia ordinaria o del carácter rector de lo teórico con respecto de tal experiencia. Esta función regulativa dirige la actividad real e histórica de construcción de sistemas de conceptos e hipótesis y, por tanto, de teorías. ${ }^{28}$ Por ende, gracias a esa función las teorías que en cierta época son tenidas por las mejores codeterminan inevitablemente y en gran parte lo que los objetos empíricos son para los sujetos de esa época. ${ }^{29}$ Disiento de Krausser en este último punto porque, como adelanté, entiendo que esa codeterminación no es inevitable, sino que, aunque usualmente efectuada, es en última instancia sólo posible.

Terminaré esta Sección con una indicación acerca del carácter indeterminado de las dimensiones objetiva y trascendental que acabo de presentar. En la "Analítica trascendental" de la primera Crítica (KrV, A64/B89 ss.) se intenta demostrar, entre otras cosas, que doce categorías son condiciones de posibilidad de la experiencia en general. Pero la exigencia regulativa de introducir conceptos teóricos carece de correlato objetivo o trascendental determinado. No es posible determinar ni a priori ni necesariamente qué conceptos teóricos adquirirán cierta validez objetiva y serán entonces condiciones de posibilidad de una experiencia científica, podrán ser así incluidos en los contenidos lógicos de los conceptos empíricos y desempeñar consiguientemente un papel como condiciones de posibilidad de la experiencia de objetos empíricos en tanto objetos empíricos de ciertas clases. Más aún, la exigencia regulativa de introducir conceptos teóricos, como se señaló, no es en sentido estricto necesaria para la experiencia mencionada en último lugar. Tal exigencia regulativa está hasta ahora justificada únicamente en la medida en que es necesaria para la posibilidad del tipo de ciencia presentado más arriba. Por tanto, su carácter necesario depende del carácter necesario de tal clase de ciencia. Esta cuestión será tratada más ampliamente en la Sección 2.

El estudio del uso hipotético regulativo de la razón y del requerimiento regulativo de sistematicidad que se efectuará en la Sección 2 permitirá, en primer lugar, completar la explicación del sentido en el que las leyes pertenecientes al tipo de ciencia consideradofundamentalmente en el AtD pueden adquirir una necesidad regulativa ${ }^{30} \mathrm{y}$, en segundo lugar, concluir la explicación del papel que desempeñan los conceptos empíricos como condiciones de posibilidad de la experiencia de objetos empíricos como objetos de ciertas clases. Se mostrará también que una de las funciones regulativas de la exigencia de sistematicidad es una condición de la aplicación del requerimiento regulativo de introducir conceptos teóricos. ${ }^{31}$

\footnotetext{
${ }^{27}$ Los conceptos de experiencia que mencioné, a saber, "experiencia científica" y "experiencia en tanto que requiere conceptos empíricos", son diferentes del concepto de experiencia que es decisivo en la "Analítica trascendental" (KrV, A64/B89 ss.), esto es, "enlace sintético de intuiciones empíricas de acuerdo con las categorías." Por tanto, las condiciones de posibilidad de los dos primeros tipos mencionados de experiencia son "trascendentales" en un sentido diferente del sentido en el que las condiciones de posibilidad de la experiencia en general lo son. Acerca de la indeterminación de las dos primeras condiciones de posibilidad, véase infra.

${ }^{28}$ Krausser remite en este punto a $\mathrm{KrV}$, A834/B862

${ }^{29}$ Cf. Krausser 1987: 183-184.

${ }^{30}$ Cf. supra, n. 9 y Sección 2 (esp. n. 46).

${ }^{31}$ La lectura de McFarland en su conjunto, en cambio, coincide en general con los resultados de mi exposición hasta este momento. Según McFarland, lo regulativo es una condición del conocimiento científico y sólo puede mostrarse que, si no es presupuesto, la investigación científica se torna imposible (McFarland 1970: 18, 22-23, 29, 36-37, 42 y 88).
} 
2. El uso hipotético regulativo de la razón y el requerimiento regulativo de sistematicidad. Sus exigencias metodológicas y su dimensión objetiva

Algunas líneas después del pasaje en el que introduce la noción de concepto teórico, pasaje que comenté al inicio de la primera Sección, Kant presenta los conceptos de uso apodíctico y uso hipotético de la razón. Según el uso apodíctico de la razón, se debe partir de una regla universal que esté dada y sea cierta, a fin de determinar necesariamente los casos particulares que le corresponden. Pero existe también un uso de la razón diferente, esto es, el hipotético:

lo universal es supuesto sólo problemáticamente, y es una mera idea; lo particular es cierto, pero la universalidad de la regla para esa consecuencia es todavía un problema; entonces, varios casos particulares, que son todos ciertos, se ensayan con respecto a la regla [para ver] si se siguen de ella; y en ese caso, si parece ser que todos los casos particulares que puedan darse se siguen de ella, se infiere la universalidad de la regla, y a partir de ésta, después, se infieren también todos los casos que en sí mismos no están dados. (KrV, A646-647/B674-B675).

El uso hipotético de la razón presupone la existencia constatada de ciertos casos particulares. Se supone problemáticamente una ley empírica universal de la cual estos casos podrían seguirse, a fin de explicarlos en tanto consecuencias de la misma. Según Kant, los juicios problemáticos son formulados con la conciencia de su mera posibilidad, a saber, sin ser tenidos por verdaderos ni falsos, y su condición principal es la ausencia de contradicción. ${ }^{32}$ Por otro lado, al inicio del pasaje citado se afirma que lo universal supuesto problemáticamente "es una mera idea". Tal expresión indica, a mi parecer, que la ley empírica universal, postulada según el uso hipotético de la razón, debe emplear uno o más conceptos teóricos. ${ }^{33}$ Por tanto, puede afirmarse que este uso hipotético entraña la consideración inicial de hipótesis de leyes empíricas científicas como meramente posibles. $^{34}$

Krausser mostró convincentemente que en los textos kantianos pueden encontrarse cinco criterios según los cuales las hipótesis empíricas científicas deben ser admitidas y evaluadas. A diferencia de la interpretación aquí expuesta, Krausser considera esas hipótesis en tanto que emplean principalmente conceptos empíricos. Aquí sólo puede presentarse un resumen de tales criterios. 1) El objeto postulado por la hipótesis como fundamento de explicación no debe estar en desacuerdo con las condiciones de la experiencia. ${ }^{35}$ 2) El fundamento de explicación postulado por una hipótesis debe estar conectado con fenómenos dados y según al menos una ley empírica. ${ }^{36}$ 3) Debe ser posible deducir a priori los casos particulares dados de una hipótesis a partir de esa sola hipótesis. ${ }^{37}$ 4) Debe ser posible deducir a priori a partir de una hipótesis casos particulares que no estaban dados cuando la hipótesis fue formulada. ${ }^{38} 5$ ) Una hipótesis no debe precisar el apoyo de hipótesis auxiliares (esto es, ad hoc). En caso de que las precise, debe emplear la menor cantidad posible de ellas. ${ }^{39}$ Aunque Krausser considera casi exclusivamente las hipótesis que emplean sólo conceptos empíricos, aquí nos

32 "El mundo puede existir por ciego azar" es un ejemplo de juicio problemático (cf. KrV, A74-75/B100-101 y Log, AA 09: 108109).

${ }^{33}$ Según Rajiva, la expresión "mera idea" puede referirse a los aquí llamados "conceptos teóricos" o al principio regulativo de sistematicidad de la razón (Rajiva 2006: 117-119). Aquí es preciso subrayar que el uso hipotético de la razón se aplica, o al menos puede aplicarse, a hipótesis que emplean conceptos teóricos. El hecho de que la expresión "mera idea" puede referirse a conceptos teóricos queda establecido porque la afirmación, según la cual en el uso hipotético de la razón lo "universal es supuesto sólo problemáticamente, y es una mera idea", se encuentra en el texto kantiano inmediatamente después del pasaje en el que se introduce la noción de "concepto teórico" (KrV, A646/B674).

${ }^{34}$ Presenté el tipo de ciencia del cual se ocupa principalmente el AtD en la Sección 1.

${ }^{35}$ Cf. KrV, A770-771/B798-799 y Log, AA 09: 85.

${ }^{36} \mathrm{Cf}$. KrV, A770-B798 y A772/B800.

${ }^{37}$ Cf. KrV, B115, A774/B802 y Log, AA 09: 84-85

${ }^{38}$ Cf. KrV, A646-647/B674-675, A790-791/B818-819 y Log, AA 09: 84-85.

${ }^{39}$ Cf. KrV, B115, A774/B802 y Log, AA 09: 85-86. Para una explicación de estos cinco criterios, cf. Krausser (1987: 167-178) y Santos García (2004: 207-208). 
interesa únicamente la aplicación de estos criterios a las hipótesis que también emplean conceptos teóricos, ya que estas últimas son las consideradas principalmente en el AtD. Para aplicar esos criterios a estas hipótesis sólo es necesario reformular el primer criterio de la siguiente manera: el objeto teórico postulado por la hipótesis como fundamento de explicación debe ser pensado como contenido en objetos empíricos que estén de acuerdo con las condiciones de la experiencia. ${ }^{40}$

Las tres últimas exigencias metodológicas no son sólo criterios de admisión de hipótesis, sino también criterios de valoración comparativa de las mismas. Mientras menos hipótesis auxiliares necesite una hipótesis, más probable será; mientras más casos dados o no dados al momento de formularse la hipótesis puedan deducirse directamente de ella, más probable será (cf. Log, AA 09: 85). Sin embargo, aunque una hipótesis pueda tornarse más probable, ella nunca alcanzará una certeza apodíctica. A fin de concluir que una hipótesis es una verdad universal apodícticamente cierta sería necesario tener conocimiento de la verdad de todas las posibles consecuencias de esa hipótesis. Como esto no es posible en virtud de nuestra finitud, empleamos una inferencia analógica y afirmamos que, si todas las consecuencias de la hipótesis que hasta ahora conocemos son verdaderas, también serán verdaderas todas las restantes consecuencias posibles de ella. Por tanto, no es posible alcanzar una "certeza apodíctica" acerca de una hipótesis, sino tan sólo un "análogo de la certeza" [Analogon der Gewißheit] (Log, AA 09: 84-85). ${ }^{41} \mathrm{Al}$ contrario, la extracción de una sola consecuencia falsa a partir de una hipótesis basta para considerar esta última como falsa (KrV, A791/B819).

De todas maneras, las mencionadas tres últimas exigencias del uso hipotético regulativo de la razón, en tanto que conducen a la explicación de una o más clases de fenómenos dados y no dados a partir de una hipótesis de ley única, introducen un requerimiento de economía o simplicidad en la explicación científica. Este requerimiento también está presente, como se vio en la Sección 1, en la exigencia regulativa de introducir conceptos teóricos. Estas exigencias son entonces conjuntamente condiciones de la aplicación del principio regulativo de unidad sistemática de la razón teórica, dado que establecen una tendencia a la unidad en la explicación de la multiplicidad de los fenómenos. A continuación se expondrá el mencionado principio regulativo de unidad sistemática.

Al inicio de AtD1 se brinda una caracterización de las ideas de la razón que es válida fundamentalmente para estas ideas en tanto principios (los de homogeneidad, especificación y continuidad, expuestos en AtD1 $)^{42}$ y en tanto entes de razón (los del alma, el mundo y Dios, expuestos en AtD2). ${ }^{43}$ Estas dos clases de ideas están relacionadas con la exigencia de unidad sistemática de la razón teórica. Esta razón emplea regulativamente las mencionadas ideas para establecer la exigencia de conseguir la unidad y extensión sistemáticas absolutas de los conocimientos empíricos. Pero como un sistema absoluto de tales conocimientos sólo puede darse parcialmente en nuestra experiencia finita, este sistema puede únicamente desempeñar el papel de meta inalcanzable para todas las posibles acciones empíricas del entendimiento. De esta manera, la función regulativa de la razón teórica impone al entendimiento, como tarea necesaria, la consecución asintótica de la unidad y extensión sistemáticas completas del conocimiento empírico (cf. KrV, A642/B670 ss.).

La exigencia regulativa de sistematicidad lleva a la formación de diferentes clases de sistemas de conocimientos empíricos. Por un lado, están los sistemas clasificatorios jerárquicos de conceptos teóricos y empíricos. Por el otro, está cuando menos el sistema explicativo jerárquico de leyes empíricas científicas. ${ }^{44}$ Quisiera señalar que estos sistemas son interdependientes. En primer

${ }^{40}$ Acerca de las leyes científicas que emplean conceptos teóricos, véase la Sección 1. Sobre la física matemática, su parte pura y su carácter sistemático, véase también la Sección 1. Acerca de las generalizaciones inductivas, cf. supra, n. 18.

${ }^{41}$ Cf. KrV, A646-647/B674-675 y A790-791/B818-819.

${ }^{42}$ Cf. KrV, A657-658/B685-686 y A664-666/B692-694.

${ }^{43} \mathrm{Cf} . \mathrm{KrV}, \mathrm{A} 670-673 / \mathrm{B} 698-701$.

${ }^{44}$ Como adelanté, el problema de las leyes empíricas ordinarias en su especificidad no será examinado en profundidad en este trabajo. Tampoco me ocupo aquí del problema de las leyes pertenecientes a la física matemática. Cf. supra, n. 16, n. 17, n. 18, n. 39 e infra, n. 46 
lugar, para formular leyes empíricas científicas se deben poseer conceptos de los objetos teóricos y empíricos a los cuales ellas se refieren. En segundo lugar, las propiedades causales que las leyes empíricas científicas atribuyen a los objetos teóricos y empíricos se incluyen en los contenidos de los conceptos teóricos y pueden incluirse en los de los conceptos empíricos. ${ }^{45}$

La conexión entre la exigencia regulativa de sistematización y el uso hipotético regulativo de la razón se pone de manifiesto, según entiendo, en A647/B675. Allí se afirma: "[e]1 uso hipotético de la razón se dirige, pues, a la unidad sistemática de los conocimientos propios del entendimiento; y ésta, a su vez, es la piedra de toque de la verdad de las reglas". Estimo que, según la primera oración citada, el uso hipotético de la razón, tal como selo expuso hasta ahora, conduce a la aplicación del principio regulativo de unidad sistemática o, como se dijo antes, es una condición de esta aplicación. La segunda oración citada, a mi parecer, puede interpretarse en el sentido de que la posibilidad de integrar un concepto o una hipótesis de ley en el sistema correspondiente es uno de los criterios que se deben tener en cuenta para determinar la adecuación del concepto o el valor de verdad de la hipótesis de ley. ${ }^{46} \mathrm{Si}$ bien se señaló que una hipótesis no puede ser verdadera en el sentido de alcanzar certeza apodíctica, la integrabilidad en un sistema de una hipótesis de ley que emplee conceptos teóricos y haya sido puesta a prueba empíricamente con éxito permite atribuirle a ella una necesidad que se podría llamar 'regulativa'. Puede afirmarse que la exigencia regulativa de sistematicidad, en la medida en que se ejerce sobre hipótesis de leyes, es un criterio más para la evaluación de las mismas, aunque presupone los cinco anteriores y es más complejo que ellos. ${ }^{47}$

La complejidad de la exigencia regulativa de sistematicidad consiste en que su ejercicio presupone necesariamente el empleo de las ideas de la razón en tanto principios y en tanto entes de razón. A continuación expondré estas clases de ideas en el orden en el que las mencioné. Presentaré entonces, en primer lugar, las ideas como principios, muy sucintamente y, por motivos de espacio, únicamente en tanto que conducen a la formación de sistemas de conceptos.

El principio de homogeneidad establece la necesidad de buscar la identidad de la especie en los diversos objetos empíricos singulares y la identidad del género en diferentes conceptos específicos

${ }^{45}$ Cf. las discusiones de Allison y Geiger (Allison 2001: 31; Geiger 2003: 276). Cf. también KrV, A664/B692.

${ }^{46}$ Cf. KrV, A651/B679; Krausser (1987: 180-181 y 184-185); Santos García (2004: 207) y (Abela 2006: 419 ss.).

47 "Incluso las leyes de la naturaleza, si se las considera como principios del uso empírico del entendimiento, llevan consigo, a la vez, una expresión de necesidad, y por tanto, al menos la sospecha de una determinación a partir de fundamentos que son válidos $a$ priori y antes de toda experiencia. Pero todas las leyes de la naturaleza, sin diferencia, están sometidas a principios superiores del entendimiento, pues ellas sólo los aplican a éstos a casos particulares del fenómeno" (KrV, A159/B198. Cf. KrV, B165 y A216/B263). "Esta idea [MA: de una totalidad del conocimiento] postula, según eso, una unidad completa del conocimiento que es propio del entendimiento, en virtud de la cual ese [conocimiento] no se constituye como un mero agregado contingente, sino que llega a ser un sistema interconectado según leyes necesarias" (KrV, A645/B673). El segundo pasaje citado puede interpretarse en el sentido de que sólo las leyes empíricas científicas que formen parte del sistema total del conocimiento empírico podrían ser consideradas como necesarias. Este sistema total, como señalé, es una meta inalcanzable que orienta necesariamente al accionar del entendimiento. Una ley de esa clase, esto es, que emplea conceptos teóricos, puede ser tenida por necesaria en caso de que sea posible integrarla en un sistema de esa clase de leyes que no haya sido falsado, porque así es posible suponer que esa ley podría llegar a ser incluida en el sistema completo del conocimiento empírico. No obstante, el sistema de leyes del cual la ley en cuestión pasaría a formar parte podría ser falsado en el futuro, o ser remplazado por otro sistema que provea más explicaciones o explicaciones más precisas. Por tanto, en última instancia, las leyes empíricas científicas no pueden adquirir, en nuestra experiencia finita, más que lo que se podría denominar un "análogo de necesidad". Esta necesidad puede llamarse "regulativa", porque sólo puede ser alcanzada, en sentido estricto, en el marco del sistema total del conocimiento empírico que la función regulativa de la razón teórica impone como meta que se debe perseguir asintóticamente. Las leyes empíricas científicas pueden alcanzar grados de precisión, simplicidad y potencial explicativo considerablemente mayores que los de las generalizaciones inductivas, porque las primeras emplean conceptos teóricos y son integrables en sistemas estrictamente coherentes. La experiencia ordinaria, por el contrario, no implica necesariamente ni una coherencia ni una conexión estrictas entre las diversas generalizaciones inductivas. Por tanto, estas generalizaciones ni siquiera pueden ser supuestas como pertenecientes al sistema completo del conocimiento empírico. Cf. supra, n. 16, n. 17, n. 18, n. 19 y n. 39. Coincido, entonces, con Buchdahl cuando sostiene que el principio de sistematicidad y el requerimiento de introducir los aquí denominados "conceptos teóricos" son conjuntamente condiciones de una cierta necesidad de las leyes empíricas científicas que se podría llamar "empírica" o "regulativa". Sin embargo, Buchdahl no expone en detalle las propiedades de esas dos prescripciones metodológicas y de sus interrelaciones, y enfatiza unilateralmente la exigencia de sistematicidad (cf. Buchdahl 1965, 1967 y 1969). Otras discusiones enfatizan exclusivamente o aún más unilateralmente la conexión entre la exigencia de sistematicidad y una cierta necesidad de las leyes empíricas (cf. Buchdahl 1971; Kitcher 1986 y Krausser 1987). Por último, otros intérpretes enfatizan unilateralmente la exigencia regulativa de introducir conceptos teóricos (cf. Brittan 1992 y McNulty 2015). La compleja interpretación de McNulty no puede analizarse aquí en detalle por motivos de espacio. 
teóricos y empíricos (KrV, A651-654/B679-682).$^{48}$ La aplicación de este principio conduce así a la máxima 'unidad' sistemática posible de los conceptos teóricos y empíricos. Debe notarse que la búsqueda de identidad entre conceptos de órdenes cada vez más elevados puede detenerse en caso de que se alcance un género supremo, esto es, un concepto que ya no pueda ser entendido como una especie de otro concepto más genérico.

El principio de especificación plantea el requerimiento de buscar la división de cada género en especies diferentes y de cada especie en subespecies diferentes (KrV, A654-657/B682-685). Siempre es posible encontrar entre los casos particulares de un cierto concepto, además de las propiedades comunes a todos ellos (propiedades cuyas representaciones discursivas y universales constituyen el contenido de ese concepto), conjuntos de diferencias significativas que permitan, por ejemplo, conceder validez objetiva a dos posibles especies de tal concepto. ${ }^{49}$ Por tanto, el principio de especificación puede aplicarse indefinidamente y conduce así a la máxima 'extensión' sistemática posible de los conceptos teóricos y empíricos..$^{50}$

El principio de continuidad conecta las exigencias de máxima unidad genérica y máxima extensión de contenido, porque establece el requerimiento de que, entre dos especies de un mismo género que se consideren inicialmente como colindantes, se introduzcan cada vez más especies intermedias. Una especie intermedia introducida de esta manera es diferente de la primera y de la segunda en menor medida que estas dos últimas entre sí. El principio de continuidad, por tanto, tiende a la producción de un tránsito continuo entre las diversas especies de cada uno de los géneros $(\mathrm{KrV}$, A657-661/B685-689). ${ }^{51}$ Este principio puede aplicarse indefinidamente, porque, según Kant, desde el punto de vista de la razón no puede haber especies "inmediatas unas a otras" ( $\mathrm{KrV}, \mathrm{A} 659 / \mathrm{B} 687)$.

El concepto posible que expresa la identidad de contenido de dos o más conceptos del mismo orden (homogeneidad), la división del contenido de un concepto (especificación) o la continuidad relativa entre especies de un concepto (continuidad) es creado por la razón teórica. Tal concepto permanece posible hasta que el entendimiento, así orientado por la razón y en vinculación con la sensibilidad, pueda concederle ciertavalidez objetiva mediante mera observación (concepto empírico) o experimentación (concepto teórico). ${ }^{52}$ En A643/B671 Kant afirma: "[1]a razón no se refiere nunca directamente a un objeto, sino solamente al entendimiento [...] [pues] ella no crea conceptos (de objetos), sino que solamente los ordena". Aunque este pasaje parezca contradecir la tesis según la cual la razón puede crear conceptos de objetos, estimo que esta facultad, al ejercer su actividad sistematizadora, crea conceptos posibles a fin de rellenar los 'huecos' que quedan en el sistema de los conceptos de objetos existentes y establecer así relaciones entre estos últimos conceptos. Como solamente el entendimiento, vinculado con la sensibilidad, puede conceder validez

${ }^{48}$ Dado que los conceptos y objetos teóricos son postulados conjuntamente por la razón, no puede haber objetos teóricos sin conceptos teóricos que les correspondan. Por otro lado, véanse los ejemplos de aplicación del principio de homogeneidad en el ámbito de la química en KrV, A652-653/B680-681. Allí afirma Kant, por ejemplo, que los químicos redujeron "todas las sales a dos géneros principales, las ácidas y las alcalinas" e intentaron también reducir esta diversidad a un género único.

${ }^{49}$ Según la razón, ninguna especie puede considerarse "como la [especie] ínfima" (KrV, A655/B683). Por otro lado, a fin de encontrar conjuntos de diferencias significativas entre los casos particulares de un concepto teórico, no deben examinarse únicamente los correspondientes objetos teóricos contenidos en diversos objetos empíricos, porque esos objetos teóricos son, por definición, idénticos. Es preciso examinar también los objetos empíricos en los cuales esos objetos teóricos están contenidos. Tales objetos empíricos deben ser estudiados solamente en la medida en que contienen los objetos teóricos relevantes, y a fin de obtener indicios acerca de los mencionados conjuntos de diferencias significativas.

${ }^{50}$ Véase el ejemplo perteneciente a la química presentado por Kant en KrV, A657/B685. Allí se menciona allí el descubrimiento de que las tierras absorbentes son de dos especies (tierras calcáreas y muriáticas).

${ }^{51}$ Kant presenta el ejemplo de aplicación del principio de continuidad a las leyes del movimiento de los cuerpos celestes $(\mathrm{KrV}$, A662-663/B690-691). Goldberg propone un ejemplo de aplicación de este principio a los conceptos aquí llamados teóricos teniendo en cuenta un estado de la ciencia posterior al de la época de Kant. Si la mente poseyera los conceptos de carbono-12 y carbono-14, la aplicación del mencionado principio llevaría a la producción del concepto posible de carbono-13 (cf. Goldberg 2004: 407 e infra)

${ }^{52}$ Coincido en este punto con Goldberg y Rauscher, aunque Goldberg no distingue entre conceptos teóricos y empíricos y Rauscher no enfatiza suficientemente esta distinción (cf. Goldberg 2004: 407 y 419 y Rauscher 2010: 295-297). 
objetiva a esos conceptos posibles, esta interpretación no contradice la tesis kantiana según la cual la razón no se refiere nunca 'directamente' a un objeto, sino al entendimiento.

Anteriormente se mostró que, en general, la exigencia regulativa de introducir conceptos teóricos es una condición de la aplicación del principio regulativo de unidad sistemática de la razón, porque establece una tendencia a la unidad en la explicación de los fenómenos. El análisis del principio regulativo de sistematicidad permite concluir, por otro lado, que este principio puede llevar al diseño de novedosos experimentos que ponen a prueba hipótesis que emplean conceptos teóricos.

En AtD2 Kant presenta una tríada diferente de ideas de la razón, a saber, la de las ideas como 'entes de razón'. Tales entes de razón no son objetos existentes, sino el contenido temático de las ideas en tanto conceptos, considerado como un objeto o, tal vez más exactamente, cuasi-objeto. Hay tres de estos entes de razón: los del alma, el mundo y Dios. ${ }^{53}$ Según Kant, podemos y tenemos que representar de manera sistemática los objetos empíricos en la medida en que los referimos a tales entes de razón.

La concepción de las ideas de AtD2 y de su relación con la de AtD1 presentan una complejidad que las hace merecedoras de un estudio independiente. Aquí me limitaré a resumir las conclusiones al respecto que alcancé en un trabajo previo. ${ }^{54}$ En ese trabajo coincidí con Caimi y Allison en que las concepciones de las ideas de AtD1 y AtD2 pueden integrarse en una teoría coherente, en la medida en que las ideas como entes de razón se entienden como condiciones de la aplicación de las ideas como principios. Además, procuré poner al descubierto los modos en los que las ideas como entes de razón orientan y determinan la aplicación de las ideas como principios. ${ }^{55}$

Las ideas presentadas en AtD2, a diferencia de las ideas como principios, no se aplican a la totalidad de los conceptos empíricos y teóricos y de las leyes empíricas científicas que los emplean, sino a los fenómenos y desde un determinado punto de vista. ${ }^{56}$ El ente de razón de la idea de alma hace necesario alcanzar la más completa unidad sistemática posible de las determinaciones de la mente, en la medida en que estas últimas se conciben como potencias o cambios de estado de la misma substancia simple y permanente y, entonces, como diferentes de los fenómenos externos ${ }^{57} \mathrm{La}$ referencia de todos los fenómenos al ente de razón de la idea de mundo hace necesario que en la explicación de ellos (esto es, en la conceptualización de los mismos y su sometimiento a leyes empíricas) se proceda como si sus series de estados de ellos fuesen infinitas. ${ }^{58}$ Por último, el ente de razón de la idea de Dios conduce necesariamente a concebir todo ordenamiento del mundo como si hubiera surgido del designio de una razón suprema y, por tanto, como conforme a fines [zweckmäßig]. Gracias a esta presuposición pueden hacerse descubrimientos de acuerdo con la causalidad mecánica que de otro modo no hubieran sido notados. ${ }^{59}$

Mencionaré dos ejemplos de determinación de la aplicación de las ideas como principios mediante las ideas como entes de razón. Como las ideas en tanto principios contienen ya en sí mismas una exigencia de sistematización, podría preguntase, por ejemplo, en qué medida la aplicación de estos principios a los fenómenos del sentido interno requiere como su condición el empleo del ente de razón de la idea del alma. El punto es que las exigencias de sistematización de las ideas como principios no implican necesariamente que pueda encontrarse una unidad sistemática de la

${ }^{53}$ Una caracterización de las ideas como entes de razón es ofrecida por Caimi (cf. Caimi 1996: 76 ss.). La expresión "cuasi-objeto" [Quasi-Gegenstand] es introducida por Zocher (cf. Zocher 1958: 48)

${ }_{54}$ Cf. Arias Albisu 2012.

${ }^{55}$ Cf. Caimi 1995: 319 y Allison 2004: 438-439. Morrison presenta una tesis similar (cf. Morrison 1989: 164-166). La divergencia entre las concepciones de las ideas de AtD1 y AtD2 es subrayada por Zocher (cf. Zocher 1958: 58 y 1966: 225).

${ }^{56} \mathrm{Cf}$. KrV, A681/B709 y supra, n. 39. La sistematización de los fenómenos afecta a los conceptos empíricos y a los conceptos teóricos de elementos puros contenidos en esos fenómenos. Además, los sistemas de conceptos y de leyes son interdependientes.

${ }^{57} \mathrm{Cf} . \mathrm{KrV}, \mathrm{A} 672 / \mathrm{B} 700$ y A682-684/B710-712.

${ }^{58} \mathrm{Cf} . \mathrm{KrV}, \mathrm{A} 672 / \mathrm{B} 700$ y A684-685/B712-713.

${ }^{59} \mathrm{Cf} . \mathrm{KrV}, \mathrm{A} 672-673 / \mathrm{B} 700-701$ y A685/B713 ss 
experiencia interna. Esta exigencia ulterior de unidad sistemática de los fenómenos del sentido interno, que orienta la aplicación de las ideas como principios a estos fenómenos, es posible por el ente de razón de la idea del alma. Sin esta orientación ulterior, la aplicación del principio de homogeneidad podría conducir a la infructuosa tarea de buscar homogeneidad entre una facultad de la mente y un objeto en el espacio. Por otro lado, como no puede haber experimentación en el ámbito de la experiencia interna (cf. supra, n. 20), el empleo del ente de razón de la idea de alma tiene también la utilidad negativa de invalidar los intentos de formular hipótesis que empleen conceptos teóricos referidos a objetos teóricos contenidos en fenómenos internos que deban ser sometidas a experimentación.

Por su parte, el ente de razón de la idea de mundo exige que, para, por ejemplo, considerar una ley causal que explique ciertos cambios de estado, no apliquemos el principio de homogeneidad sólo a los cambios de estado de los fenómenos dados actualmente a los sentidos, sino que también examinemos cambios de estado documentados del pasado y continuemos estudiándolos en el futuro, sin terminar nunca esta búsqueda.

Concluiré esta Sección con una reflexión acerca de la dimensión objetiva y trascendental de las exigencias metodológicas del uso hipotético regulativo de la razón y del principio regulativo de sistematicidad. Como se adelantó en la Sección 1, las tres ideas de la razón en tanto principios son, según Kant, principios lógicos o metodológicos que requieren una fundamentación provista por los tres principios trascendentales que les corresponden. Explicaré esta cuestión resumiendo las tesis de Caimi acerca de ella (cf. Caimi 1995: 311-317). Sería irracional e injustificado buscar la unidad sistemática de los conocimientos empíricos exigida por los principios lógicos de homogeneidad, especificación y continuidad, si no se presupusiera a priori que la mencionada unidad pertenece a los objetos empíricos mismos. Tal presuposición de sistematicidad "como perteneciente a los objetos mismos" (KrV, A650/B678) se torna necesaria en virtud de los principios trascendentales de homogeneidad, especificación y continuidad. Estos principios trascendentales, por su parte, no son legítimos porque sirvan de fundamento para los principios lógicos, dado que estos últimos podrían ser arbitrarios. Tales principios son trascendentales, en cambio, porque son condiciones de posibilidad de la experiencia de los objetos empíricos como objetos de ciertas clases. ${ }^{60}$ Por ejemplo, si no se presupusiera, de acuerdo con el principio trascendental de homogeneidad, que los objetos empíricos que tienen la misma apariencia tienen también la misma constitución interna, no sería posible abstraer conceptos empíricos a partir de tales objetos, ${ }^{61} \mathrm{y}$, como se señaló, sin conceptos empíricos no es posible la experiencia de objetos empíricos en tanto objetos empíricos de determinadas clases. ${ }^{62}$

La exigencia regulativa de sistematicidad tiene entonces una dimensión objetiva e incluso trascendental. Esta dimensión es indeterminada. Afirma Kant, por ejemplo, que de acuerdo con el principio trascendental de homogeneidad presuponemos necesariamente homogeneidad en lo múltiple de la experiencia, "aunque no podamos determinar a priori el grado de ella" (KrV, A654/B682). Esta afirmación significa que, aunque tenemos que presuponer a priori la unidad sistemática del conocimiento en la naturaleza misma, no podemos determinar a priori el grado de unidad sistemática que encontraremos en la naturaleza en un momento dado. ${ }^{63}$

La dimensión objetiva del requerimiento regulativo de sistematicidad que se acaba de exponer corresponde fundamentalmente a ese requisito en tanto que concierne a los conceptos

${ }^{60} \mathrm{Cf} . \mathrm{KrV}, \mathrm{A} 650-651 / \mathrm{B} 678-679$, A653-654/B681-682, A656-657/B684-685 y A660-661/B688-689.

${ }^{61}$ Cf. KrV, A653-654/B681-682 y EEKU, AA 20: 215-216.

${ }^{62}$ Acerca de los distintos conceptos de "experiencia" y "estatus trascendental", véase supra, n. 26. Dado que las ideas como entes de razón son condiciones de la aplicación de las ideas como principios, la función trascendental de las últimas requiere el empleo de las primeras.

${ }^{63} \mathrm{Cf}$. KrV, A653/B681, A665/B693 y A668/B696. Wartenberg entiende la distinción entre principios lógicos y trascendentales como una distinción entre principios metodológico-regulativos y el conocimiento a priori e indeterminado incorporado en los mismos (cf. Wartenberg 1979: 414-424). 
empíricos. Sin embargo, aquí me ocupo de las leyes empíricas más tenidas en cuenta, más bien, en el AtD, a saber, las leyes empíricas científicas que emplean conceptos teóricos. Con respecto a este último punto, mostraré, en primer lugar, que existe una conexión entre, por un lado, la exigencia regulativa de introducir conceptos teóricos y las tres últimas exigencias del uso hipotético regulativo de la razón y, por el otro, el principio regulativo de sistematicidad. En segundo lugar, expondré la conexión que existe entre los mencionados requisitos relacionados con los conceptos teóricos y el requisito de sistematicidad referido a los conceptos empíricos.

En la Sección 1 se mostró que las leyes empíricas científicas requieren el empleo de conceptos teóricos para explicar precisa y económicamente cierta(s) clase(s) de fenómenos. Además, al comienzo de la Sección presente se indicó que las tres últimas exigencias del uso hipotético regulativo de la razón tienden a introducir unidad en la explicación de los fenómenos. De este modo, estas prescripciones conducen a la formación de hipótesis de leyes que, en virtud de las características mencionadas y a diferencia de las generalizaciones inductivas, pueden ser integradas en sistemas de leyes estrictamente coherentes (cf. supra, n. 46). Como se señaló, el principio regulativo de sistematicidad exige la formación de sistemas cada vez más unificados y extensos de los conocimientos empíricos. Si dispusiéramos sólo de generalizaciones inductivas y no tuviéramos leyes con potencial explicativo amplio y económico, entonces el principio regulativo de sistematicidad no podría formar un sistema de leyes empíricas científicas que sea estrictamente coherente y además suministre una explicación económica de una gran variedad de fenómenos. ${ }^{64}$ Por otra parte, como se afirmó, la formación de este sistema y la formación de un sistema de conceptos teóricos son interdependientes.

Al final de la Sección 1 se vio que los conceptos teóricos son condiciones de posibilidad de lo que se podría llamar una 'experiencia científica'. Además, estos conceptos pueden ser condiciones de posibilidad de la experiencia de objetos empíricos como objetos empíricos de ciertas clases, en la medida en que tales conceptos teóricos sean incluidos como notas discursivas en el contenido lógico de conceptos empíricos. Sin embargo, como se indicó en la Sección 1, es posible que tenga lugar una experiencia ordinaria sin la intervención de conceptos teóricos. ${ }^{65}$ Las tesis sobre los conceptos teóricos expuestas en este párrafo valen también para las leyes empíricas científicas.

En conclusión, si bien la exigencia de introducir conceptos teóricos, las tres últimas exigencias del uso hipotético de la razón y la exigencia de sistematicidad referida a los conceptos teóricos y las leyes que los emplean conducen conjuntamente a la formación de un sistema de leyes empíricas científicas, tales exigencias no poseen una dimensión objetiva propia más amplia que la que acabo de exponer, a saber, la consistente en una experiencia científica.

Cabe señalar que estas exigencias vinculadas con los conceptos teóricos sólo pueden aplicarse bajo el presupuesto de la aplicación de la exigencia de sistematicidad referida a los conceptos empíricos. Anteriormente se mostró que la última exigencia es una condición de posibilidad de los conceptos empíricos. Y, ciertamente, no es posible formar, por ejemplo, un concepto teórico que represente un objeto teórico cuya función sea esclarecer los procesos de calcinación y combustión sin poseer previamente conceptos empíricos tales como los de plomo y de carbón. Sin tales conceptos empíricos, sería imposible identificar todos los objetos empíricos correspondientes a fin de formar el mencionado concepto teórico.

Por último, quisiera distinguir dos tesis presentadas en los dos últimos párrafos. Los conceptos empíricos hechos posibles por la exigencia de sistematicidad referida a ellos son condiciones de posibilidad de la formación de los conceptos teóricos. Una vez que se han formado

${ }^{64}$ Por otro lado, como ya indiqué, el requerimiento de sistematicidad puede conducir al diseño de experimentos que ponen a prueba hipótesis que utilizan conceptos teóricos.

${ }^{65}$ Cf. supra, n. 23, n. 24 y n. 26. 
tales conceptos teóricos, es posible incluirlos como notas discursivas en el contenido lógico de los conceptos empíricos.

\section{Conclusiones}

En la Sección 1 se estudió la exigencia regulativa de introducir conceptos teóricos. Mostré allí que la clase de disciplina considerada con más atención en el AtD, esto es, al menos, la química ${ }^{66}$ está integrada por leyes empíricas científicas que se distinguen por su empleo de conceptos teóricos. Por otro lado, indiqué, en primer lugar, que la exigencia mencionada tiene una dimensión objetiva y trascendental con respecto a una experiencia científica. En segundo lugar, señalé que la exigencia en cuestión puede entenderse como condición de posibilidad de la experiencia de objetos empíricos como objetos empíricos de ciertas clases, en la medida en que los conceptos teóricos sean incluidos como notas discursivas en el contenido lógico de conceptos empíricos. Sin embargo, es posible que haya una experiencia que involucre conceptos empíricos que no posean como notas discursivas conceptos teóricos. Por último, mostré que la exigencia regulativa de introducir conceptos teóricos tiene una dimensión objetiva indeterminada. No puede establecerse a priori qué conceptos teóricos obtendrán cierta validez objetiva y serán así condiciones de posibilidad de una experiencia científica, ni tampoco cuáles de estos conceptos objetivamente válidos podrán ser integrados en los contenidos lógicos de conceptos empíricos y ser entonces condiciones de posibilidad de la experiencia de objetos empíricos como objetos empíricos de determinadas clases.

En la Sección 2 se mostró que la exigencia regulativa de introducir conceptos teóricos, las tres últimas exigencias del uso hipotético regulativo de la razón y la exigencia regulativa de sistematicidad referida a los conceptos teóricos y las leyes que los emplean son condiciones de posibilidad de la formación de un sistema de leyes empíricas científicas poseedoras de una necesidad 'regulativa', así como de la formación de un sistema de conceptos teóricos. Por tanto, estas tres exigencias mencionadas son conjuntamente condiciones de posibilidad de una experiencia científica. Además, pueden ser condiciones de posibilidad de la experiencia de objetos empíricos como objetos empíricos de ciertas clases en el sentido explicado en el párrafo anterior.

Otra conclusión importante de la Sección 2 es que el requerimiento regulativo de sistematicidad relativo a los conceptos empíricos es condición de posibilidad de estos conceptos. Esta dimensión objetiva de tal requerimiento es, como afirmé, también indeterminada. Además, los conceptos empíricos son condiciones de posibilidad de los conceptos teóricos. Considero que los aportes fundamentales del AtD acerca de la dimensión objetiva de la función regulativa de la razón teórica son los dos siguientes: i) la especificación de las condiciones que deben cumplir las leyes empíricas para adquirir la necesidad regulativa que las convierte en leyes científicas que pueden pertenecer, al menos, a la química. Estas condiciones son el empleo de conceptos teóricos y la integrabilidad en sistemas de leyes estrictamente coherentes y de un poder explicativo amplio, preciso y económico. Las leyes que poseen tal necesidad regulativa son condiciones de posibilidad de una experiencia científica y, como se vio, 'pueden' ser asimismo condiciones de posibilidad de la experiencia de objetos empíricos como objetos empíricos de ciertas clases. ii) La caracterización de la exigencia regulativa de sistematicidad relativa a los conceptos empíricos como condición de posibilidad de los conceptos empíricos y, por tanto, de la experiencia de objetos empíricos en tanto

\footnotetext{
${ }^{66}$ Como indiqué en la Sección 1, no dispongo de espacio para examinar el problema de si la clase de ciencia que expuse en este artículo está, de acuerdo con el pensamiento de Kant en el período crítico, integrada sólo por la química o incluye también otras disciplinas
} 
objetos empíricos de ciertas clases y, como también señalé, de los conceptos teóricos y las leyes que los emplean. ${ }^{67}$

Por último, estimo que mi exposición pone al descubierto la falencia más importante de las discusiones contenidas en el AtD, a saber, la ausencia de una fundamentación de la experiencia científica que vaya más allá del señalamiento según el cual las leyes empíricas científicas suministran explicaciones unificadas referidas a amplios conjuntos de fenómenos.

Bibliografia

ABELA, Paul: “The Demands of Systematicity: Rational Judgment and the Structure of Nature", en BIRD, G. (ed.): A Companion to Kant, Oxford/Malden, Blackwell, 2006.

ALliSON, Henry E.: Kant's Theory of Taste, Cambridge, Cambridge University Press, 2001.

ALLISON, Henry E.: Kant's Transcendental Idealism. Revised and Enlarged Edition, New Haven/London, Yale University Press, 2004.

ARIAS ALBISU, Martín: “Acerca de la relación entre los dos tipos de esquemas de las ideas de la razón en la Crítica de la razón pura de Kant”, Areté 24:1 (2012) 7-24.

ARIAS ALBISU, Martín: "Las prescripciones metodológicas de la función regulativa de la razón teórica en la 'Crítica de la razón pura'de Kant", Kant e-Prints 10:1 (2015) 64-93.

BENNETT, Jonathan: Kant's Dialectic, Cambridge, Cambridge University Press, 1974.

BRITTAN, Gordon G.: "Systematicity and Objectivity in the Third Critique", The Southern Journal of Philosophy 30:S1 (1992) 167-186.

BUCHDAHL, Gerd: "Causality, Causal Laws and Scientific Theory in the Philosophy of Kant", The British Journal for the Philosophy of Science 16 (1965) 187-208.

BUCHDAHL, Gerd: "The Relation between 'Understanding' and 'Reason' in the Architectonic of Kant's Philosophy”, Proceedings of the Aristotelian Society 67 (1967) 209-226.

BUCHDAHL, Gerd: "The Kantian 'Dynamic of Reason', with Special Reference to the Place of Causality in Kant's System", en BECK, L. W. (ed.): Kant Studies Today, La Salle, Open Court, 1969

BUCHDAHL, Gerd: “The Conception of Lawlikeness in Kant's Philosophy of Science”, Synthese 23 (1971) 24-46

BUCHDAHL, Gerd: "Gravity and Intelligibility: Newton to Kant", en BUCHDAHL, G. (ed.): Kant and the Dynamics of Reason, Oxford, Blackwell, 1992.

BUTTS, Robert E.: "The Methodological Structure of Kant's Metaphysics of Science", en BUTTS, R. E. (ed.): Kant's Philosophy of Physical Science, Dordrecht, D. Reidel, 1986.

CAIMI, Mario: "Über eine wenig beachtete Deduktion der regulativen Ideen", Kant Studien 86 (1995) 308-320.

CAIMI, Mario: “La función regulativa del ideal de la razón pura”, Diánoia 42 (1996) 61-79.

CARRIER, Martin: "Kant's Theory of Matter and His Views on Chemistry”, en WATKINS, E. (ed.): Kant and the Sciences, Oxford, Oxford University Press, 2001.

FRIEDMAN, Michael: Kant and the Exact Sciences, Cambridge, Harvard University Press, 1992.

GEIGER, Ido: "Is the Assumption of a Systematic Whole of Empirical Concepts a Necessary Condition of Knowledge?", Kant Studien, 94 (2013) 273-298.

${ }^{67}$ Véase el último párrafo de la Sección 2. 
GOLDBERG, Nathaniel J.: "Do Principles of Reason Have 'Objective but Indeterminate Validity'?”, Kant Studien 95 (2004) 405-425.

KANT, Immanuel: Kant's gesammelte Schriften, Deutsche (anteriormente Königlich Preußische) Akademie der Wissenschaften (ed.), Berlin y otros, Walter de Gruyter y predecesores, 1900ss. (Akademie-Ausgabe=AA).

KANT, Immanuel: Lógica. Un manual de lecciones. Edición original de G. B. Jäsche, traducción castellana de M. J. Vázquez Lobeiras, Madrid, Akal, 2000 (Log).

KANT, Immanuel: Crítica de la razón pura, traducción castellana de M. Caimi, México, Fondo de Cultura Económica, UAM, UNAM, 2009 (KrV, A/B).

KANT, Immanuel: Primeros principios metafisicos de la ciencia de la naturaleza, traducción castellana de S. Nemirovsky, México, Universidad Nacional Autónoma de México, 1993 (MAN). 165

KEMP SMITH, Norman: A Commentary to Kant's "Critique of Pure Reason". Second edition, revised and enlarged, New York, Palgrave Macmillan, 1923/2003.

KITCHER, Philip: "Projecting the Order of Nature", en BUTTS, R. E. (ed.): Kant's Philosophy of Physical Science, Dordrecht, D. Reidel, 1986.

KRAUSSER, Peter: "Über den hypothetischen Vernunftgebrauch in der Kritik der reinen Vernunft", Archiv für Geschichte der Philosophie 69 (1987) 164-196.

McFARLAND, John D.: Kant's Concept of Teleology, Edinburgh, University of Edinburgh Press, 1970.

McNULTY, Michael B.: "Rehabilitating the Regulative Use of Reason: Kant on Empirical and Chemical Laws", Studies in History and Philosophy of Science 54 (2015) 1-10.

MORRISON, Margaret: "Methodological Rules in Kant's Philosophy of Science", Kant Studien 80 (1989), 155-172.

NAYAK, Abhaya C. y SOTNAK, Eric: "Kant on the Impossibility of the 'Soft Sciences", Philosophy and Phenomenological Research 55 (1995) 133-151.

PATON, Herbert J.: Kant's Metaphysic of Experience, London, George Allen \& Unwin Ltd., 1936/1970.

RAJIVA, Suma: "Is Hypothetical Reason a Precursor to Reflective Judgment?", Kant Studien 97 (2006) 114-126.

RAUSCHER, Frederick: "The Appendix to the Dialectic and the Canon of Pure Reason. The Positive Role of Reason", en GUYER, P. (ed.): The Cambridge Companion to Kant's Critique of Pure Reason, Cambridge, Cambridge University Press, 2010.

SANTOS GARCÍA, Miguel Á.: "Kant y la lógica de la investigación científica”, Teorema 23 (2004) 199-213.

SLOAN, Philip R.: "Kant on the history of nature: The ambiguous heritage of the critical philosophy for natural history", Studies in History and Philosophy of Biological and Biomedical Sciences 37 (2006) 627-648.

STAHL, Georg E.: Zufällige Gedancken und nützliche Bedencken über den Streit von dem sogenannten Sulphure, Halle, Wäysenhaus, 1718.

WARTENBERG, Thomas E.: “Order through Reason. Kant's Transcendental Justification of Science”, Kant Studien 70 (1979) 409-424.

WARTENBERG, Thomas E.: "Reason and the Practice of Science", en GUYER, P. (ed.): The Cambridge Companion to Kant, Cambridge, Cambridge University Press, 1992. 
ZOCHER, Rudolf: "Zu Kants transzendentaler Deduktion der Ideen der reinen Vernunft”, Zeitschrift für philosophische Forschung 12 (1958) 43-58.

ZOCHER, Rudolf: “Der Doppelsinn der kantischen Ideenlehre. Eine Problemstellung”, Zeitschrift für philosophische Forschung 20 (1966) 222-226. 


\title{
Formas de autoconciencia en la "Reflexión de Leningrado"
}

\author{
MATÍAS HERNÁN OROÑO
}

\begin{abstract}
Resumen
El objetivo principal de este trabajo es señalar que los aspectos novedosos del manuscrito conocido como "Reflexión de Leningrado" no se hallan en el tratamiento de la auto-afección, ni en los argumentos contra el idealismo cartesiano, sino en el desarrollo de distintas formas de autoconciencia que están íntimamente ligadas a la conciencia de la propia corporalidad.
\end{abstract}

Palabras clave: autoconciencia, Reflexión de Leningrado, apercepción cosmológica, ente del mundo [Weltwesen], corporalidad

\section{Forms of self-awareness in the "Leningrad Reflection"}

\section{Abstract}

The main aim of this paper is to point out that the novel aspects of the manuscript known as "Leningrad Reflection" are neither in the treatment of self-affection nor in the arguments against Cartesian idealism, but in the development of different forms of self-awareness that are closely linked to the awareness of the own embodiment.

Keywords: self-awareness, Leningrad Reflection, cosmological appreception, being in the World [Weltwesen], embodiment

\section{Introducción}

La reflexión titulada "Vom inneren Sinne", también conocida como "Reflexión de Leningrado" $(\mathrm{RL}){ }^{2}$ fue redactada aproximadamente entre los años 1786 y 1790 . En este manuscrito se encuentran diferentes líneas argumentativas mediante las cuales Kant intenta justificar que el sentido interno presupone necesariamente el sentido externo. Esta misma tesis fue defendida en algunos textos que pertenecen a la segunda edición de la Crítica de la razón pura (KrV). Me refiero a la denominada "Refutación del idealismo" (KrV, B 274-B 279) y a los textos dedicados al problema de la autoafección ( $\mathrm{KrV}$, B 67 ss.; B 154 ss.). En líneas generales, es posible sostener que la auto-afección supone la actividad del entendimiento que se dirige hacia un múltiple espacial, mientras que la estrategia implementada contra el idealista que pone en duda la existencia del mundo exterior consiste en subrayar que la sucesión de representaciones ante el sentido interno sólo puede constituirse mediante la referencia a algo permanente que se encuentra en el espacio. De esta manera, el

${ }^{1}$ Universidad de Buenos Aires - CONICET

${ }^{2}$ Se denomina "Reflexión de Leningrado" a un manuscrito que fue hallado a mediados de la década del '80 del siglo XX en la biblioteca Saltykov-Shchedrin de Leningrado. Se publicó por primera vez en el año 1986 en la revista Voprosy Filosofii (4, 1986, 126-136) bajo el título Loses Blatt Leningrad 1 (cf. Jáuregui 2008: 158; Zoeller 1989: 264; La Rocca 2003: 54). De aquí en adelante utilizaré las siglas RL para referirme a este texto. Indicaré con números romanos el número de página y con números arábigos el número de línea correspondiente al manuscrito original. 
manuscrito de Leningrado "Vom inneren Sinne" presentaría una mera repetición de ideas que fueron formuladas en la segunda edición de la primera Crítica.

Frente a esta posible interpretación de la RL que minimiza la presencia de aportes novedosos, en este artículo intentaré defender las siguientes tesis: i) la RL presenta una novedad de alto impacto que excede el planteo de la "Refutación del idealismo" y el de la doctrina de la autoafección; ii) en la RL se presenta la distinción entre distintas formas de autoconciencia (cf. Zoeller 1989: 267) ${ }^{3}$, dentro de las cuales se destacan la apercepción cosmológica y la conciencia de sí como ente del mundo [Weltwesen]; iii) la apercepción cosmológica constituye un modo de autoconciencia empírica que toma en consideración la propia existencia corpórea; iv) la conciencia de uno mismo como ente del mundo [Weltwesen] también toma en cuenta la propia corporalidad, pero se sitúa en el plano de una autoconciencia originaria e inmediata que es identificada con la intuición del espacio.

En primer término, realizaré una breve referencia a aquellas partes de la RL que simplemente reformulan la doctrina de la auto-afección y los argumentos contra el idealismo problemático. Esto permitirá comprender que las tesis sobre la auto-afección y la refutación del idealismo desarrolladas en el manuscrito no implican novedades sustanciales. En segundo lugar, analizaré las dos formas novedosas de autoconciencia que aparecen en RL. En este punto será de particular interés el estudio de la denominada 'apercepción cosmológica' y su distinción respecto a la conciencia de sí como ente del mundo [Weltwesen].

\section{Los pasajes sobre la auto-afección y la refutación del idealismo}

Considero que el texto puede ser dividido en tres partes. La primera sección se ocupa del problema de la auto-afección (RL, I, 1-21), la segunda introduce la distinción entre diversas formas de autoconciencia (RL, I, 22 - II, 5) y la tercera parte retoma la polémica contra el idealismo problemático (RL, II, 6-32). En este primer apartado comentaré brevemente la primera y la tercera de las secciones aquí mencionadas. Gracias a este análisis será posible concluir que estos pasajes constituyen reformulaciones de tesis ya planteadas por Kant — principalmente, aunque no únicamente, en la segunda edición de la $\mathrm{KrV}$ - y en ese sentido no aportan elementos sustancialmente novedosos al cuerpo de su teoría crítica.

En la primera sección (RL., I, 1-21), Kant retoma el oscuro problema de la auto-afección al afirmar que sólo si se presupone la aprehensión de una multiplicidad externa es inteligible la determinación empírica del sentido interno que se denomina auto-afección. Es decir, el aparecer fenoménico del yo bajo la forma sucesiva del sentido interno supone la descripción del espacio y la aprehensión de su multiplicidad. Kant destaca la relación de dependencia entre el sentido interno y el sentido externo, pues "[...] me afecto a mí mismo al llevar las representaciones de los sentidos externos a la conciencia empírica de mi estado" (RL., I, 10-11). Esta primera sección de la RL finaliza de la siguiente manera: "[...] no tendríamos un sentido interno y no podríamos determinar nuestra existencia [Daseyn] en el tiempo si no tuviésemos un sentido externo real y no nos representáramos objetos en el espacio como diferenciados de nosotros.” (RL., I, 18-21)

\footnotetext{
${ }^{3}$ Mi interpretación se halla en contraposición con la efectuada por María Luisa Couto-Soares, quien incluso afirma: "no vamos a encontrar en este texto nada radicalmente nuevo o sorprendente: la reflexión contiene una reiteración de los argumentos para la refutación del idealismo, que Kant había expuesto ya en otras reflexiones - concretamente en las conocidas bajo el nombre de los ejercicios de Kiesewetter - y, evidentemente, en los textos de la $2^{\mathrm{a}}$ edición de la Crítica, la nota al Prefacio de esta edición y el texto introducido al final de la Analítica" (Couto-Soares 2004: 842). No obstante, la autora luego expresa una opinión contraria al subrayar que la RL presenta dos tópicos que están ausentes en las otras obras de Kant: la serie modal del tiempo "yo fui, yo soy y yo seré" y la conciencia a priori de objetos externos (cf. Couto-Soares 2004: 846). Estos dos tópicos son los que en el presente trabajo serán reconstruidos bajo la forma de 'apercepción cosmológica' y conciencia a priori de sí como ente del mundo (Weltwesen).
} 
Independiente de la claridad que puedan aportar estas líneas a la comprensión del problema de la auto-afección, estas no contribuyen con elementos realmente novedosos. ${ }^{4}$ Aquí simplemente queda subrayada de manera explícita la tesis de que no podríamos poseer un sentido interno si no tuviésemos un sentido externo real.

Por su parte, en la tercera parte del manuscrito (RL., II, 6-32), Kant establece una serie de argumentos contra el idealismo problemático de raigambre cartesiana. En un primer momento (RL., II, 6-13), sostiene que la intuición misma del espacio prueba que algo en general existe fuera de mí y que ser afectado presupone necesariamente la existencia de algo externo. Este tipo de argumentos parece suponer aquello que debe ser demostrado. Se trata de un modo de argumentación similar a la que se presenta en el contexto del "Cuarto paralogismo" (KrV, A 367-380), donde se afirma que la mera percepción de algo externo es una prueba de la realidad de aquello que es percibido. En un segundo momento (RL., II, 14-32), Kant desarrolla una reducción al absurdo de la posición del idealista problemático. El argumento expuesto puede ser reconstruido del siguiente modo:

i. Las meras representaciones de nuestro sentido interno se dan de manera sucesiva (ausencia de permanencia en el sentido interno) [premisa tácita, la cual sin embargo ya habría sido afirmada unas líneas antes al sostener que el percipiens "[...] sólo puede acontecer sucesivamente, es decir, hacia delante [...]]” (RL. I, 30).

ii. A partir del mero sentido interno no se puede derivar la representación del espacio, la cual supone algo permanente (RL., II, 16-22).

iii. Soy consciente de representaciones de cosas espaciales (es decir, representaciones de algo permanente) como pertenecientes al sentido interno (RL., II, 28-29).

Por tanto, es imposible que no haya ningún sentido externo (caso contrario, nuestras representaciones de cosas en el espacio serían ellas mismas espaciales, lo cual es absurdo) (RL., II, 30-32).

Aquí se sostiene que sólo suponiendo un sentido externo, en el cual puede ubicarse algo permanente, es posible dar cuenta de aquellas representaciones que acontecen en el sentido interno. Así pues, la estrategia kantiana consiste en tomar como punto de partida las representaciones meramente subjetivas, acerca de cuya existencia el idealista problemático no puede dudar. Dentro de estas representaciones encontramos algunas que se presentan como espaciales (como se afirma en la tercera premisa), las cuales sólo son posibles si se supone la existencia de un sentido externo real, pues no es posible derivar la permanencia a partir de una sucesión de representaciones. Si se negara la realidad del sentido externo, se anularía el punto de partida del idealista problemático, a saber: la realidad de las representaciones como estados de conciencia que se dan sucesivamente en el sentido interno. Por un lado, la representación de algo permanente sería ininteligible si solamente se supone la realidad del sentido interno. Kant está sosteniendo con este argumento la imposibilidad de reducir la espacialidad al sentido interno, ello es así puesto que a este último le falta el rasgo de la permanencia. Por otro lado, estas representaciones (entre las cuales nos representamos algunas cosas como permanentes) se dan en un orden sucesivo, el cual como ya fue demostrado en la "Refutación del idealismo" (KrV, B 274-278), sólo es posible en virtud de algo permanente y, por tanto, espacial. ${ }^{5}$ Ahora bien, el argumento contra el idealismo cartesiano presente en la RL no sólo señala que la sucesión se constituye como tal en virtud de la representación de algo permanente, sino que además subraya que las representaciones de algo permanente son irreducibles al orden de la sucesión propio del sentido interno. Si se diera el caso de que estas representaciones se explicaran en virtud del mero

${ }^{4}$ La doctrina de la auto-afección es desarrollada principalmente en los $\S \S 8$ y 24 de la KrV. También aparecen algunos elementos en el $§ 24$ de la Antropología en sentido pragmático (Anth, AA 07: 161.06-162.11).

${ }^{5}$ H. Robinson (1989: 277) sostiene que "antes" y "después" no tienen ningún sentido si no se supone la espacialidad del mundo exterior. 
sentido interno, el idealista problemático debería conceder que las representaciones mismas existen como algo en el espacio, lo cual es absurdo. Pero aun aceptando esta cuestión, el argumento kantiano presenta ciertos problemas, pues la representación de algo espacial determinado no implica necesariamente la existencia de esa cosa determinada en el espacio. Un claro ejemplo sobre esta última cuestión lo constituyen los estados de conciencia pertenecientes a la fantasía, la alucinación y los sueños. Así pues, considero que la representación de un determinado objeto espacial X no implica necesariamente su referencia a un determinado objeto X que existe en el espacio. Es cierto que la representación de algo permanente supone la existencia de un sentido externo 'en general', pues en tanto representación del sentido interno, ésta forma parte de una sucesión que requiere de lo permanente en el espacio. Pero no creo que la representación de un objeto 'determinado' con características espaciales implique, necesariamente, su existencia en el espacio.

A partir del análisis recién efectuado, considero que este argumento contra el idealismo problemático introducido en la tercera sección de la RL no aporta elementos valiosos para refutar el idealismo que pone en duda la realidad del mundo externo. Si lo que el argumento quiere probar es que la sucesión supone la permanencia, puesto que la primera sólo puede constituirse mediante la referencia a la segunda, entonces nos hallamos ante un argumento que ya estaba presente en la "Refutación del idealismo". Pero como ya he señalado, el argumento contra el escepticismo acerca del mundo externo que Kant desarrolla en RL no reposa únicamente sobre la dependencia de la sucesión respecto de la permanencia, sino que allí se intenta reducir al absurdo la posición del escéptico sosteniendo que si el sentido externo no fuese real, entonces nuestras representaciones de cosas espaciales serían ellas mismas, en tanto representaciones, espaciales (lo cual es absurdo).

Ante este panorama cabe preguntarse cuáles son los aspectos novedosos que se introducen en el manuscrito de la RL, pues ni el tratamiento de la auto-afección ni el de la refutación del idealismo aquí desarrollados constituyen piezas argumentales que merezcan la pena ser destacadas. A continuación intentaré ofrecer una respuesta a este interrogante. Para ello será necesario abordar la segunda parte del texto (RL, I, 22 - II, 5).

\subsection{La percepción empírica o cosmológica}

En primer lugar, se formula la noción de apercepción pura, trascendental o apperceptio percipientis. Es un modo de autoconciencia que permite afirmar "[...] yo soy" (RL., I, 23) y constituye el "[...] fundamento de toda percepción y de toda experiencia" (RL., I, 8-9). Sin lugar a dudas, se trata de una referencia a la apercepción trascendental, tal como fue tematizada en la $\mathrm{KrV}$. No obstante, es importante destacar que aquí no se formula un "yo pienso" (como ocurría en $\mathrm{KrV}$, B 132), sino un "yo soy". Una posible interpretación de este cambio en la formulación del principio de la apercepción trascendental podría entenderse del siguiente modo: nos hallamos ante la explicitación de una nota contenida en el "yo pienso" de la apercepción trascendental, la cual implica la existencia de un sujeto que piensa y acompaña a cada una de sus representaciones. El sujeto trascendental es un yo que piensa. De allí que Kant formule la apperceptio percipientis bajo la forma de un "yo soy". ${ }^{6}$ Por otro lado, encontramos la noción de apercepción empírica, apercepción cosmológica o apperceptio percepti. Mediante esta última podemos afirmar "[...] yo fui, yo soy y yo seré, en otras palabras, yo soy una cosa del tiempo pasado, presente y futuro [...]" (RL, I, 23, 24). ${ }^{7}$

\footnotetext{
${ }^{6}$ Ciertamente, la interpretación que aquí ofrezco es altamente discutible. En la literatura sobre el principio de la apercepción trascendental, la cual se expresa mediante el "yo pienso", se discute si este "yo" es un sujeto efectivamente existente. Mario Caim (2014: 106) sostiene que "[...] el yo de la fórmula de la apercepción tiene una función rigurosamente determinada y enteramente formal, que no debe ser confundida con las funciones de un yo efectivamente existente [...]". La interpretación desarrollada por Caimi se halla en contraposición a la interpretación de Dieter Henrich (1976: 83), quien llega a afirmar que el yo de la apercepción es "un individuo efectivamente existente". Ofrecer una argumentación rigurosa acerca del pasaje del "yo pienso" (KrV, B 132) al "yo soy" (RL, I, 23) exigiría una investigación minuciosa sobre el tema, la cual excede el marco de este trabajo.

${ }^{7}$ Guenter Zoeller (1989: 266) sostiene que la expresión cosmologische Apperception junto con su formulación "ich war, ich bin, und ich werde seyn" no se halla en ninguna otra parte del corpus kantiano.
} 
La apercepción cosmológica es caracterizada como un tipo de autoconciencia que subraya el horizonte temporal de pasado, presente y futuro de la conciencia empírica. Todo lo que acontece en el tiempo es un fenómeno, por tanto este yo que es, fue y será, sólo puede ser dado como fenómeno, es decir, empíricamente. Implícitamente queda vedado el acceso a lo que el yo es en sí mismo, con independencia de las formas de la sensibilidad. Asimismo, la autoconciencia empírica puede ser descripta como aquello que aparece de manera sucesiva, en la medida en que es caracterizada como “[...] una cosa del tiempo pasado, presente y futuro [...]” (RL., I, 23-24). A continuación se añade:

La apercepción cosmológica, la cual considera mi existencia como magnitud en el tiempo [Größe in der Zeit] me coloca en relación con otras cosas que existen, existieron y existirán, pues la simultaneidad no es una determinación de lo real con respecto al percipiens sino al perceptum, ya que toda percepción en la simultaneidad sólo es representada en lo que puede ser percibido hacia atrás (en vista del tiempo pasado), así como también, hacia adelante, lo cual no puede ser la existencia del percipiens, el cual solo puede acontecer sucesivamente, en otras palabras, hacia adelante [...] (RL, I, 25-30).

Aquí puede observarse que la apercepción empírica o cosmológica coloca al yo en relación con otras cosas que existen, existieron o existirán. Según Kant, esto se debe a que la simultaneidad sólo puede ser representada en objetos espaciales, en los cuales es indiferente el orden de las percepciones. Asimismo, parece estar supuesta en este contexto la dependencia de la sucesión respecto de la simultaneidad. Dicho en otros términos, sólo podemos determinarnos como magnitudes que despliegan su existencia temporal sucesiva si suponemos un orden de objetos espaciales y simultáneos, gracias a los cuales la sucesión es posible. Unas líneas más adelante Kant sostiene que el objeto exterior "[...] es requerido para la autoconciencia empírica (del ser simultáneo), y por lo tanto, lo conozco de la misma manera en que me conozco a mí mismo." (RL, II, 2-4). ${ }^{8}$ Por un lado, se afirma la conciencia acerca de la simultaneidad empírica entre objetos exteriores al yo. Por otro lado, queda en evidencia la autoconciencia empírica de simultaneidad, es decir, aquella que se establece entre la propia existencia y los objetos percibidos. De esta manera, quedan determinadas en primer lugar, la espacialidad o exterioridad tanto de los objetos percibidos como del yo fenoménico y, en segundo lugar, la autoconciencia empírica de las relaciones de simultaneidad entre ambos términos (el yo y los objetos espaciales que percibimos). Por lo tanto, es posible afirmar que mediante la apercepción cosmológica es tematizada la autoconciencia empírica de la simultaneidad entre la propia existencia y el mundo de objetos espaciales. De allí que nos encontremos ante un tipo de conciencia empírica que revela el yo fenoménico ya no como un mero fluir de representaciones que se suceden en el sentido interno, sino como el aparecer del yo en el espacio, el cual establece relaciones de coexistencia o simultaneidad con otros cuerpos también espaciales. ${ }^{9}$ Considero que este modo de autoconciencia ya aparecía tratado de una manera tangencial en el siguiente pasaje de la "Tercera analogía de la experiencia":

Es fácil notar, en nuestras experiencias, que sólo los influjos continuos en todos los lugares del espacio pueden dirigir nuestro sentido de un objeto al otro; que la luz, que juega entre nuestro ojo y los cuerpos del mundo, pone en obra una comunidad mediata entre nosotros y ellos, y así demuestra la simultaneidad de los últimos; que no podemos mudar empíricamente ningún lugar (percibir esa mudanza) sin que por todas partes la materia nos haga posible la percepción de nuestro lugar; y [que] ella sólo por medio de su recíproco influjo puede exhibir (aunque sólo mediatamente) su simultaneidad, y mediante ello, la coexistencia de ellos, hasta los más remotos objetos. (KrV, A 213/ B 260)

\footnotetext{
${ }^{8}$ El subrayado es propio.

${ }^{9}$ Según Manfred Baum (1989: 281-282) la noción de apercepción cosmológica nos recuerda la concepción leibniziana del alma como vis representativa universi, en la medida en que sólo podemos representarnos el mundo como un contenido de nuestra alma concebida como una fuerza representativa del universo. Por momentos, Kant parece adherir a esta idea al sostener que sólo nos podemos conocer en la medida en que conocemos el mundo. Sin embargo, Baum añade que la semejanza con la metafísica de Leibniz es limitada, pues el sistema de armonía preestablecida de Leibniz representa el tipo de teoría que Kant intenta refutar al sostener el carácter necesario del sentido externo y su imposibilidad para ser deducido a partir del sentido interno.
} 
Aquí se afirma que la luz que media entre los cuerpos del mundo y el ojo, revela una comunidad mediata entre ambos. Que la materia de los objetos simultáneos manifieste de manera indirecta la comunidad entre el yo como ser corpóreo y dichos objetos puede explicarse mediante el siguiente ejemplo: si en un tiempo 1 me hallo a la derecha de una 'silla roja' y a la izquierda de una 'silla negra' y en un momento 2 intercambio las posiciones de las sillas, entonces me encontraré a la derecha de la 'silla negra' y a la izquierda de la 'silla roja'. Es decir, la mudanza de los objetos simultáneos revela de manera mediata la simultaneidad entre ellos y el propio cuerpo, pues no es posible introducir una mudanza entre los objetos exteriores sin que sea al menos posible la percepción de una mudanza del propio cuerpo en relación con tales objetos.

La categoría de comunidad o acción recíproca supone fenómenos que son 'percibidos' en el espacio como simultáneos. Es decir, la comunidad de objetos presupone relaciones espaciales entre ellos. A su vez, tales relaciones sólo son posibles si los cuerpos vinculados se hallan 'en el espacio', como objetos del sentido externo (i.e. si son cuerpos o al menos puntos en el espacio). Por tanto, el tipo de subjetividad que aquí entra en comunidad con los cuerpos del mundo debe ser una subjetividad emplazada en el espacio, es decir, una subjetividad arraigada a un cuerpo. En el marco de la "Tercera Analogía de la experiencia" se afirma que la simultaneidad entre distintos fenómenos se establece de manera objetiva gracias al concepto de comunidad. Esta comunidad o causalidad recíproca entre los objetos es paralela a una comunidad entre los diversos objetos situados empíricamente en el espacio y el propio cuerpo. Por lo tanto, quedaría establecida de manera objetiva no sólo la simultaneidad 'empírica' entre los objetos exteriores, sino además la simultaneidad 'empírica' entre los objetos y el propio cuerpo como magnitud que ocupa un lugar en el espacio. ${ }^{10}$

Retomemos ahora el análisis sobre la RL. En relación con la noción de 'apercepción cosmológica' no queda muy claro el motivo por el cual Kant introduce el modo temporal de la simultaneidad a fin de hacer inteligible la sucesión del sentido interno, sobre todo si se tiene en cuenta que para ello hubiese bastado con señalar la relación entre sucesión y permanencia, tal como fue establecido en la "Primera Analogía de la experiencia" (KrV, A 182 / B 224 y ss.) y en la "Refutación del idealismo" (KrV, B 274 - B 279). Considero que la clave para comprender este desplazamiento hacia el modo temporal de la simultaneidad radica en que no se quiere simplemente demostrar los supuestos de la sucesión en tanto tal, sino desarrollar un argumento que permita dar cuenta de aquel modo de autoconciencia (apercepción cosmológica) que "[...] considera mi existencia como magnitud en el tiempo [Größe in der Zeit] [...]” (RL., I, 25-26). Kant está sugiriendo aquí que sólo se puede ser consciente de la propia existencia en un tiempo determinado si se toma en cuenta la inserción del yo en un mundo espacial donde interactúa con otros fenómenos también espaciales. La 'magnitud en el tiempo' [Größe in der Zeit] que se constituye mediante relaciones de simultaneidad (y por tanto, suponiendo la interacción causal recíproca), no es la magnitud constituida por una sucesión de representaciones que se suceden ante el sentido interno, sino la magnitud del yo que posee un cuerpo cuya existencia dura un tiempo determinado. Es este cuerpo propio que se constituye como una magnitud en el tiempo el que requiere establecer relaciones de simultaneidad con otras magnitudes espaciales a fin de constituirse como tal. Así pues, mientras que en el argumento de la refutación del idealismo se sostiene que la sucesión del sentido interno supone el modo temporal de la permanencia, la apercepción cosmológica considera la inserción concreta del yo en el mundo. Este auto-emplazamiento sólo es posible si el yo se considera como una magnitud espacial que interactúa con otras magnitudes también espaciales, estableciendo así relaciones de simultaneidad con el mundo. En suma, la relación entre sucesión y permanencia es suficiente para dar cuenta de la constitución del sentido interno, pero no es suficiente para garantizar la ubicación del yo en el mundo espacial. Para

${ }^{10}$ El espacio juega un rol central en el argumento de la "Tercera Analogía”, pues la determinación causal recíproca de magnitudes en el espacio sólo puede ser comprendida como una determinación recíproca de posiciones espaciales. Margaret Morrison (1998: 257-277) sostiene que la comunidad de objetos no sería posible sin presuponer relaciones espaciales entre los mismos. Expresado en otros términos, el espacio unificado es lo que nos permite concebir eventos simultáneos o sustancias coexistentes. 
esto último, es necesario introducir una nueva forma de autoconciencia que se llama 'apercepción cosmológica'. Esto es así, puesto que es al menos posible pensar sin contradicción en la posibilidad de una mente sin cuerpo, la cual podría ser consciente de una sucesión de representaciones y para ello bastaría con suponer algo permanente en virtud de lo cual tal sucesión se constituye. Por supuesto que al interior de ese orden de cosas permanentes es posible establecer relaciones de simultaneidad, pero esta supuesta mente sin cuerpo se hallaría ajena a la interacción causal recíproca y a las relaciones de simultaneidad con tales objetos espaciales. En cambio, si lo que se toma como punto de partida no es un mero flujo de representaciones que podría pertenecer a un ser pensante sin corporalidad, sino la conciencia de sí como un ser perteneciente al universo que es percibido, entonces es necesario suponer relaciones de simultaneidad no sólo entre los objetos del mundo sino entre ellos y una mente encarnada. Esto sólo es posible si el yo está emplazado en ese orden espacial.

\subsection{La conciencia de sí mismo como un ente del mundo [Weltwesen]}

Además de la apercepción pura que se expresa mediante la fórmula "[...] yo soy” (RL., I, 23) y la apercepción empírica o cosmológica que se expresa afirmando " [...] yo fui, yo soy y yo seré, en otras palabras, yo soy una cosa del tiempo pasado, presente y futuro [...]" (RL, I, 23, 24), en la RL aparece una tercera forma de autoconciencia, la cual se observa en el siguiente pasaje:

Es necesario que yo pueda ser consciente de mí mismo a priori como en relación con otras cosas, incluso antes de la percepción de ellas, por tanto, que mi intuición, corresponda como una 〈intuición〉 externa, antes de la conciencia de mi impresión, a la misma conciencia, pues el espacio es la conciencia de esta relación real. (RL, I, 34 - II, 1)

Aquí emerge un tipo de autoconciencia que no se identifica ni con la apercepción trascendental pura, ni con la apercepción empírica o cosmológica. Kant nos habla de una autoconciencia a priori que considera la propia subjetividad como si estuviera en relación con otras cosas. Es importante subrayar la expresión 'como en relación' [als in relation] en el pasaje recién citado. A diferencia de la apercepción empírica cosmológica, esta nueva forma de autoconciencia no se percata de relaciones efectivas entre el yo y los objetos espaciales, sino de la mera posibilidad a priori de establecer relaciones con el mundo espacial. Kant sostiene que la intuición de sí mismo como una intuición externa corresponde a esta autoconciencia a priori e identifica la relación entre la intuición externa de uno mismo y la percepción posible — aunque no necesariamente actualizada - de otros objetos espaciales, con la intuición pura del espacio. Es decir, la conciencia a priori del espacio implica la 'posible' autoconciencia a priori de uno mismo como si estuviese en relación con otras cosas. Además, es importante observar el modo que asume la formulación de esta autoconciencia a priori, pues Kant afirma: "Es necesario que yo pueda ser consciente de mí mismo a priori como en relación con otras cosas [...]" (RL, I, 34 - II, 1). Esto significa que 'debemos poder' ser conscientes a priori de tales relaciones de simultaneidad entre el yo espacial y los posibles objetos espaciales que conforman el mundo, ya que estas relaciones están implicadas necesariamente en la conciencia $a$ priori del espacio. Estas ideas nos ofrecen indicios para pensar en la íntima conexión entre la conciencia a priori del espacio y la conciencia de sí como un ser que entabla relaciones con posibles objetos espaciales. En continuidad con este tipo de autoconciencia a priori se sostiene lo siguiente: Yo soy inmediata y originariamente consciente de mí mismo como un ente del mundo [Weltwesens] y precisamente, sólo de esta manera es determinable mi propia existencia solamente como fenómeno, como magnitud en el tiempo. (RL, II, 4-5)

La primera parte de este enunciado: "Yo soy inmediata y originariamente consciente de mí mismo como un ente del mundo [Weltwesens]" (RL, II, 4-5) subraya el carácter a priori de la nueva 
forma de autoconciencia aquí introducida. ${ }^{11}$ Se trata de una conciencia de sí a priori, originaria e inmediata que subraya un vínculo originario entre el yo y el mundo espacial. ${ }^{12}$ Es decir, se destaca no sólo nuestro vínculo inescindible con los objetos percibidos, sino también la conciencia originaria de nuestra 'pertenencia' al mismo plano espacial y mundano, en el cual tales objetos se encuentran. ${ }^{13}$ En este punto es razonable preguntase si esta suerte de 'pertenencia' del yo al plano espacial posee el mismo estatuto que la pertenencia de los restantes objetos a un orden espacial. Considero que la respuesta es negativa, pues aquí nos encontramos frente a una autoconciencia a priori y originaria, mientras que la conciencia de objetos es empírica y supone las condiciones a priori de la experiencia. Por otro lado, creo que la autoconciencia a priori puramente intelectual indicada a través del 'yo pienso' de la apercepción trascendental no es objetivable y remite a la condición última de posibilidad de la experiencia. Análogamente, puede afirmarse que esta conciencia a priori de sí como un ente del mundo [Weltwesen] remite a una condición subjetiva que posibilita la experiencia de un orden espacial, pero ella misma no se puede constituir como un objeto en el espacio, pues se trata de una autoconciencia a priori. Nos hallamos ante un complemento de aquella apercepción pura expresada mediante el 'yo pienso'. Mientras que el 'yo pienso' es una representación intelectual y '[...] no puede ser considerada como perteneciente a la sensibilidad" ( $\mathrm{KrV}$, B 132), la conciencia originaria de sí como ente del mundo [Weltwesen] pone de manifiesto una autoconciencia a priori intuitiva (y por tanto sensible). Para dar cuenta de esta autoconciencia originaria intuitiva, Kant introduce la noción de ente del mundo [Weltwesen], la cual se identifica con la conciencia a priori del espacio.

La segunda parte del enunciado: "sólo de esta manera es determinable mi propia existencia solamente como fenómeno, como magnitud en el tiempo" (RL, II, 4-5) indica que gracias a esta autoconciencia inmediata, a priori y originaria, podemos determinar nuestra existencia como fenómeno. Es decir, la autoconciencia a priori de uno mismo como un ente del mundo [Weltwesen] posibilita o condiciona la autoconciencia empírica (ya sea aquella que permite determinar la propia existencia fenoménica en relaciones empíricas de simultaneidad con otros objetos y que aquí ha sido identificada como 'apercepción cosmológica', o bien aquella que hace abstracción de esta referencia al objeto espacial, aparece de manera sucesiva ante el sentido interno y supone la auto-afección).

Ahora bien, la identificación efectuada por el propio Kant entre la conciencia de sí como un ente mundano [Weltwesen] con la intuición pura del espacio sugiere además una distinción radical entre el modo de ser espacial de los objetos espaciales de la experiencia ordinaria y aquel ente que se identifica con la intuición pura del espacio. Si la interpretación que aquí ofrezco es correcta, debería aceptarse que ser un ente mundano [Weltwesen] no es ser un objeto del mundo, sino su condición de posibilidad. Claramente, cada sujeto puede desarrollar una perspectiva de tercera persona sobre sí mismo y convertirse en objeto de estudio desde el punto de vista de la anatomía, la fisiología, la física o la ciencia en general, pero en la medida en que efectuamos tal objetivación nos hallamos ante un caso de conciencia empírica y no ante aquella autoconciencia originaria e inmediata dirigida hacia uno mismo como ente del mundo [Weltwesen]. Dicho en otros términos, lo que no puede convertirse en objeto es la autoconciencia misma (en este caso, me refiero a la conciencia de sí como ente mundano [Weltwesen], aunque podría extenderse esta imposibilidad a otros modos de

\footnotetext{
${ }^{11}$ Una interpretación alternativa sobre esta cuestión — de la cual soy ciertamente deudor- es desarrollada por Claudia Jáuregui, quien al analizar este pasaje sostiene que nos hallamos ante la apercepción empírica o cosmológica, mediante la cual somos conscientes de la propia existencia en apertura al mundo. (Cf. Jáuregui 2008: 164-165).

${ }^{12}$ Según Manfred Baum el término Weltwesen es ambiguo, pues puede significar tanto "entidad en el mundo" como "entidad que tiene el mundo dentro de sí". Mientras que el primer sentido estaría remitiendo al cuerpo humano, el segundo se referiría al alma Esta ambigüedad es la misma que se encuentra en el primer argumento sobre el espacio en la "Estética transcendental" (KrV, B 38), así como en la "Tercera analogía de la experiencia" (KrV, B 260) (cf. Baum 1989: 284) Por mi parte, considero que es necesario subrayar que en el caso de la "Tercera analogía de la experiencia" la simultaneidad entre los objetos y el yo corpóreo es empírica, mientras que en la conciencia inmediata de sí como Weltwesen nos hallamos ante una autoconciencia a priori.

${ }^{13}$ Según Baum (1989:282) el concepto de Weltwesen anticipa en algún sentido lo que Heidegger denomina 'ser-en-el mundo' [Inder-Welt-sein]. Tanto para Kant como para Heidegger, el ser humano no sería un ente más entre otros, sino aquél que solamente puede ser para sí mismo mediante su relación con el mundo.
} 
autoconciencia). Es decir, al constituir el cuerpo humano como objeto se pierde de vista aquel modo de autoconciencia que Kant introduce al afirmar: "Yo soy inmediata y originariamente consciente de mí mismo como un ente del mundo [Weltwesens]" (RL, II, 4-5). ${ }^{14}$

Tratemos ahora de comprender cómo se inserta esta novedosa forma de autoconciencia con otros temas que aparecen tratados en el manuscrito de Leningrado sobre el sentido interno. Si bien he trazado una distinción en tres partes de la RL, considero que es posible conectar estas secciones con el objetivo de comprender de manera unitaria la argumentación allí presente. En la primera parte se retoma la doctrina de la auto-afección, señalando que ésta sólo es posible en la medida en que aprehendemos objetos en el espacio. En la segunda parte se traza una distinción entre distintas formas de autoconciencia, entre los cuales se destaca la 'apercepción cosmológica' (la cual subraya la simultaneidad empírica entre el sujeto y los objetos percibidos en el espacio) y la conciencia de sí como un ente mundano [Weltwesen], mediante la cual cada sujeto es autoconsciente de las relaciones a priori de simultaneidad que entabla con el mundo espacial. Hemos visto que esta última forma de la autoconciencia se identifica con la conciencia a priori del espacio. Por último, en lo que he identificado como una tercera parte de la RL Kant retoma la discusión con el idealismo problemático. De este modo, las tres partes del manuscrito coinciden en subrayar la tesis de que el sentido interno presupone necesariamente el sentido externo. Este último adquiere una nueva significación ya que no sólo es necesario ser conscientes de algo externo a nosotros a fin de dar cuenta del sentido interno - lo cual ya había sido demostrado de diferentes maneras en la doctrina de la auto-afección y en la refutación del idealismo problemático-, sino que ahora se identifica la conciencia a priori del espacio (sentido externo) con la conciencia de sí como ente mundano [Weltwesen]. Bajo estas consideraciones, se puede reconstruir el argumento de la RL del siguiente modo:

i. el sentido interno presupone el sentido externo.

ii. el sentido externo sólo es posible en última instancia si poseemos a priori la representación del espacio, de un modo irreducible al sentido interno.

iii. la conciencia de sí como un ente mundano [Weltwesen] implica relaciones a priori de simultaneidad entre el yo y el mundo, identificándose así con la conciencia a priori del espacio. [Weltwesen]

Por tanto, el sentido interno presupone la conciencia de sí como un ente mundano

Es decir, para dar cuenta de aquel flujo de representaciones sucesivas que aparecen ante el sentido interno es necesario suponer la realidad del sentido externo, el cual es identificado por Kant con la conciencia de sí como un ente mundano [Weltwesen], subrayando de ese modo, el 'carácter espacial $^{15}$ de uno mismo como una condición de posibilidad a priori de la experiencia externa. Considero que esta conciencia de sí como ente mundano sugiere un camino para pensar que al interior del criticismo kantiano es posible una autoconciencia a priori del cuerpo propio, la cual no es formulada explícitamente por Kant. No obstante, considero que la identificación de la conciencia de sí como ente mundano [Weltwesen] con la intuición pura del espacio (cf. RL, I, 34 - II, 1) permite vislumbrar un camino para afirmar que sólo si se posee una conciencia de sí ocupando un 'posible'

\footnotetext{
${ }^{14}$ Excede los objetivos de este trabajo indagar las consecuencias que puede tener en el proyecto crítico en su totalidad esta conciencia originaria de sí como Weltwesen. Por su parte. Angelica Nuzzo (2008) ha forjado el concepto de transcendental embodiment como un hilo conductor que atraviesa su análisis de la teoría kantiana de la sensibilidad en las tres Críticas. La autora afirma: "there is a formal side to the human body that is constitutive of our experience of rational subjects -both in our knowledge of the world and in our action in it. This formal side of our embodied condition, however, is only "visible" to (and only made visible by) a transcendental investigation" (cf. Nuzzo 2008: 8). Creo que esta idea de corporalidad formal que sólo es 'visible' mediante la investigación trascendental es semejante a la que he intentado plantear al sostener que la conciencia originaria de sí como Weltwesen debe ser interpretada como una autoconciencia originaria intuitiva que tematiza una dimensión no objetivable del cuerpo propio.

${ }^{15}$ Quizás sea mejor que hablemos de un 'carácter espacializante', a fin de distinguir la conciencia de objetos espaciales ordinarios de la conciencia originaria de sí como ente del mundo [Weltwesen].
} 
lugar en el espacio — como un ente del mundo [Weltwesen] — es posible tener experiencia externa (espacial).

\section{Conclusiones}

El manuscrito de Leningrado sobre el sentido interno excede el tratamiento de la doctrina de la autoafección y de la refutación del idealismo. Esto es así, puesto que aquí ya no se señala meramente la primacía de un mundo espacial sin el cual no sería posible el sentido interno, sino que ahora se pone de relieve que el sujeto está necesariamente en relación de simultaneidad con ese mundo espacial.

En la RL es posible distinguir cuatro formas de autoconciencia: i) la apercepción trascendental que en el marco de la KrV se expresa mediante el 'yo pienso' y en la RL adopta la fórmula 'yo soy'; ii) la conciencia de sí como ente mundano [Weltwesen]; iii) la apercepción cosmológica y iv) el yo fenoménico del sentido interno. Veamos brevemente de qué manera se diferencian estas formas de autoconciencia. En primer lugar, la apercepción trascendental pura constituye el fundamento último de toda percepción y de toda experiencia. En segundo término, la conciencia de sí como ente mundano [Weltwesen] constituye la primera innovación del manuscrito de Leningrado sobre el sentido interno. Mediante esta forma de autoconciencia el sujeto se percata de las relaciones a priori de simultaneidad que establece con el mundo. La concepción del sujeto como ente mundano [Weltwesen] permite concebir un modo de conciencia inmediata y originaria de uno mismo en relación con el mundo espacial, la cual nos indica una forma de conciencia a priori de la propia corporalidad, la cual posibilita las relaciones a priori de simultaneidad con los objetos espaciales y es identificada por Kant con la intuición del espacio. He señalado que se trata de un modo de apercepción que revela la conciencia a priori de la relación de simultaneidad de uno mismo con posibles objetos espaciales, aunque indeterminados en cuanto a su modo de existencia específica. Es como si este modo de autoconciencia sólo pudiera expresarse en términos condicionales del siguiente tipo: "si existen objetos en el espacio, esos posibles objetos deben poder hallarse a priori en relación de simultaneidad con un posible cuerpo propio". En la medida en que la conciencia de sí como ente mundano [Weltwesen] es intuitiva, considero que ella no puede ser analizada como un despliegue o un aspecto de la apercepción trascendental tal como es tratada en la $\mathrm{KrV}$ - la cual constituye un modo de conciencia puramente intelectual- Esta diferencia, entre una autoconciencia a priori intelectual y otra autoconciencia a priori intuitiva permite comprender el alcance innovador de la conciencia de sí como ente mundano [Weltwesen]. En tercer lugar, la apercepción cosmológica remite a un modo de autoconciencia mediante la cual el sujeto también se percata de las relaciones de simultaneidad entre sí mismo y los objetos espaciales, pero a diferencia de la conciencia de sí como ente mundano [Weltwesen], la apercepción cosmológica se desenvuelve en un nivel empírico. Por último, es posible identificar una cuarta forma de autoconciencia que se identifica con la conciencia empírica de uno mismo en el modo de la sucesión, haciendo abstracción de las relaciones de simultaneidad con el mundo. Esta última forma de autoconciencia es la que posibilita la conciencia de un yo en sentido psicológico y es tratada por Kant en el contexto de la auto-afección. Es importante destacar que de estas cuatro formas de autoconciencia que aquí han sido enumeradas, sólo la conciencia de sí como ente mundano [Weltwesen] y la apercepción cosmológica constituyen las innovaciones que introduce la RL y de las cuales me he ocupado en este trabajo.

Asimismo, he sostenido que la noción de ente del mundo [Weltwesen] sugiere una diferencia radical entre la conciencia de objetos espaciales ordinarios y aquella conciencia originaria e inmediata que se percata de emplazamiento subjetivo originario en el espacio. Esto implica que ser un ente del mundo [Weltwesen] no es ser un 'objeto' espacial cualquiera, pues ser un ente del mundo [Weltwesen] significa ante todo ser una 'subjetividad' autoconsciente de su identificación con la intuición pura del espacio. Queda así sugerida la presencia de una dimensión de la propia 
corporalidad que se sitúa en un plano originario, y por tanto, no se puede constituir como objeto de experiencia, sino que señala una condición de posibilidad para la conciencia de tales objetos.

\section{Bibliografia}

Obras de Immanuel Kant:

KANT, Immanuel: Kants Werke. Akademie Textausgabe, Berlin, Walter de Gruyter, 1968.

: "A New Fragment of Immanuel Kant: "On inner Sense"”, International Philosophical Quarterly 115 (1989) 249-261. Los textos citados provienen de una traducción propia.

: Crítica de la razón pura, traducción de Mario Caimi, Buenos Aires, Colihue, 2007.

Bibliografía general:

BAUM, Manfred: "Kant on Cosmological Apperception", International Philosophical Quarterly 115 (1989) 281-289.

CAIMI, Mario: "Se piensa. Sobre una función del yo en la deducción transcendental", en CAIMI, Mario (ed.): Temas kantianos, Buenos Aires, Prometeo, 2014.

COUTO-SOARES, María Luisa: "Sobre el sentido interno. La reflexión de Leningrado", Anuario Filosófico XXXVII/3 (2004) 841-850.

HENRICH, Dieter: Identität und Objektivität, Heidelberg, Carl Winter, 1976.

JÁUREGUI, Claudia: Sentido interno y subjetividad. Un análisis del problema del auto-conocimiento en la filosofía trascendental de Kant, Buenos Aires, Prometeo, 2008.

LA ROCCA, Claudio: Soggetto e mondo. Studi su Kant, Venezia, Marsilio Editori, 2003.

MORRISON, Margaret: "Community and Coexistence: Kant's Third Analogy of Experience", KantStudien 89 (1998) 257-277.

NUZZO, Angelica: Ideal Embodiment. Kant's Theory of Sensibility, Bloomington/Indianapolis, Indiana University Press, 2008.

ROBINSON, Hoke: "Inner Sense and the Leningrad Reflexion", International Philosophical Quarterly XXIX/3, 115 (1989) 271-279.

ZOELLER, Guenter: "Making Sense Out of Inner Sense: The Kantian Doctrine as Illuminated by the Leningrad Reflexion", International Philosophical Quarterly XXIX/3, 115 (1989) 263-270. 


\title{
Kants Konzeption kosmologischer Freiheit - ein metaphysischer Rest?
}

\author{
Christian KRIJNEN ${ }^{1}$
}

\begin{abstract}
Die deutschen Idealisten waren der Auffassung, Kant habe mit seiner transzendentalen Umwälzung der Begründungsproblematik eine Revolution in der philosophischen Denkungsart in Gang gesetzt, die es weniger aufzuhalten als vielmehr dadurch zu vollbringen gelte, daß die Voraussetzungen oder ,Fundamente' der Kantischen Philosophie selbst kritisch in den Blick genommen würden. Zu diesen Voraussetzungen gehört zweifelsohne Kants Architektonik der Vernunft im allgemeinen und die Stellung, die dem Freiheitsbegriff dabei zukommt, im besonderen. Es wird gezeigt, daß Kants Bestimmung kosmologischer oder transzendentaler Freiheit nicht so sehr einen sicheren Ausgangspunkt für Erkundungen im Rahmen der praktischen Philosophie etabliert als vielmehr zunächst eigens zum Thema gemacht werden sollte. Dann wird sichtbar, daß das Profil der kritischen Freiheitskonzeption Kants stark durch die deutsche Schulmetaphysik des 18. Jh.'s geprägt ist. Damit geraten allerlei Vorentscheidungen und unausgewiesene Konstellationen dieses Profils betreffend in den Blick; Vorentscheidungen und Konstellationen, die sich gemäß Hegels Analyse auch gar nicht ausweisen lassen, sondern den Übergang zu einem allgemeineren Freiheitsbegriff als dem transzendentalen oder kosmologischen Kants erforderlich machen. Die Folgen dieses Sachverhalts werden abschließend illustriert durch eine Betrachtung der Transzendentalphilosophie Bruno Bauchs, des wohl besten KantKenners des Neukantianismus.
\end{abstract}

Keywords: Bauch, Freiheit, Hegel, Kant, Metaphysik, Spontaneität, Willkür

\section{La concepción kantiana de la libertad: ¿un resto metafísico?}

\begin{abstract}
Resumen
Los idealistas alemanes entendían que Kant, con su cambio radical en la problemática de la fundamentación, puso en marcha una revolución del modo de pensar filosófico que no se debía impedir, sino completar, considerando críticamente las presuposiciones o 'fundamentos' de la filosofía crítica misma. Entre estas presuposiciones se cuenta, sin dudas, la arquitectónica kantiana de la razón en general y, en particular, la posición que le corresponde en ella al concepto de la libertad. Al respecto se señala que la determinación kantiana de la libertad cosmológica o trascendental no establece un punto de partida seguro para las investigaciones en el marco de la filosofía práctica y que primero se la debería hacer adecuada para ello. Al hacerlo, se vuelve claro que el perfil de la concepción crítica kantiana de la libertad está influenciado por la filosofía escolástica del siglo XVIII. En ese sentido, se hacen visibles varias posiciones previas y constelaciones injustificadas, correspondientes a ese perfil; posiciones y constelaciones que, de acuerdo con el análisis de Hegel, tampoco se pueden justifican, sino que hacen necesario el tránsito a un concepto de libertad más general que el de Kant. Las consecuencias de esto se ilustran mediante un examen de la filosofía trascendental de Bruno Bauch, el mayor conocedor de la filosofía de Kant en el neokantismo.
\end{abstract}

Palabras clave: Bauch, libertad, Hegel, Kant, metafísica, espontaneidad, arbitrio

1. Kosmologische Freiheit als Problem

Wenn etwas den deutschen Idealismus zusammenhält, dann der Versuch, im Ausgang von Kants Transzendentalphilosophie zu einem sowohl umfassenderen als auch tieferen Konzept von Freiheit zu

\footnotetext{
${ }^{1}$ Vrije Universitet Amsterdam.
} 
gelangen. Dieser Versuch ist mit Kants transzendentaler Wende in der philosophischen Begründungsthematik intrinsisch verbunden, besteht doch deren Sinn darin, die Vernunft, oder wie es im Diskurs auch heißt: die Subjektivität, als Quelle aller Geltung, aller Normativität menschlicher Tätigkeit und damit aller Objektivität oder Gegenständlichkeit auszuweisen. Das Denken, Tun und Lassen des Menschen gilt für Kant als Denker der Moderne nicht mehr als fremdbestimmt, heteronom, sondern als selbstbestimmt, autonom, befreit also von so oder so gearteten externen Mächten als Bestimmungsgründen. Der Begriff der Freiheit muss in einer modernen Transzendentalphilosophie folglich eine prominente Rolle spielen.

Zugleich erscheint Kants Freiheitsbegriff, so wie er historisch vorliegt (also ohne ihn mit allerlei Vor- und Nachsätzen auszustatten) unzulänglich. Den systematisch interessierten Interpreten ist schon lange aufgefallen, dass Kants Freiheitsbegriff ein praktisches Profil eignet, Kants Konzeption kosmologischer Freiheit geradezu Vorblick nimmt auf eine Metaphysik der praktischen Vernunft endlicher Subjekte. Schon im Nachweis der Denkmöglichkeit der ,kosmologischen“ Freiheitskausalität wird klar, dass es Kant vor allem um die Möglichkeit ,moralischer' Freiheit für unser ,Handeln' geht (der die kosmologische oder transzendentale Freiheit logisch vorhergeht: KrV, B561 f., vgl. 831). ${ }^{2}$ Kant modelliert Freiheit in der dritten Antinomie, zunächst die kosmologische und auf sie gründend die praktische, entsprechend als ein ,Vermögen', Ursache von Wirkungen zu sein, näherhin: als ein Vermögen, eine Reihe von Wirkungen ,von selbst', ,spontan` anzufangen. Diese vermögenstheoretische Modellierung lässt, worum es Kant geht, der schon im kosmologischen Kontext die Freiheit ,im praktischen Verstande' ins Zentrum rückt, den Menschen als Autor seiner Handlungen verstehen: nicht bloß als Wirkung einer Naturkausalität, nicht als bloßes Objekt, sondern eben als Subjekt.

Ebendieses praktische Profil der Freiheitsantinomie steht in einem durchaus schroffen Kontrast zur systematischen Problemstellung der dritten Antinomie. Kants transzendentaler Idealismus und die damit einhergehende Unterscheidung zweier Welten, der Welt als Erscheinung und der der Dinge an sich, der phänomenalen und der noumenalen Welt, macht die Komptabilität von Natur und Freiheit nicht nur einsichtig, sondern erweist das kosmologische Verhältnis beider zugleich so, dass die phänomenale Welt in der noumenalen gründet, dass Freiheit Ursache der Natur ist, die intelligible Welt die Sinnenwelt bewirkt. Freiheit im kosmologischen Verstande erweist sich als (transzendentalphilosophisch verstandener) Seinsgrund: als Grund der Erscheinungswelt.

\footnotetext{
${ }^{2}$ Für Hans Friedrich Fulda: „Freiheit als Vermögen der Kausalität und als Weise, bei sich selbst zu sein“. In: Inmitten der Zeit. Beiträge zur europäischen Gegenwartsphilosophie (FS M. Riedel), hg. von Thomas Grethlein und Heinrich Leitner (Würzburg 1996: 47-63, 55), vgl. 61, ist Kants Idee von Freiheit in Verbindung mit der allgemeinen Naturnotwendigkeit, also Kants kosmologische Freiheitsidee, vom Vorgriff auf eine Metaphysik der praktischen Vernunft endlicher Subjekte geleitet. Hans Wagner wird nicht müde zu betonen, dass es die Möglichkeit der Freiheitskausalität menschlicher Handlungen ist, die Kant in der dritten Antinomie beschäftigt (Hans Wagner: „Die kosmologische Antithetik und ihre Auflösung in Kants Kr.d.r.V“. In: Zu Kants Kritischer Philosophie, hg. von Bernward Grünewald und Hariolf Oberer, Würzburg 2008: 82-97, 85), die Auflösung der dritten Antinomie unter dem „Hauptgedanken“, dem „Hauptziel“ der Möglichkeit praktischer Freiheit steht (ebd. 95. vgl. auch Hans Wagner: „Kants ergänzende Überlegungen zur Möglichkeit von Freiheit im Rahmen der Auflösung der dritten Antinomie“. In: $Z u$ Kants Kritischer Philosophie, hg. von Bernward Grünewald und Hariolf Oberer (Würzburg 2008: 98-106, 99, 104). Vielmehr noch als um die Möglichkeit einer gegen alle Naturkausalität stehenden kosmologischen Spontan- oder Freiheitskausalität, gehe es Kant um die Möglichkeit moralischer Freiheit für menschliches Handeln (ebd. 101). „Eigentlich“ (ebd. 103) treibe Kant die Doppelnatur des Menschen als homo phaenomenon und homo noumenon, und damit als naturkausal bedingtes wie als vernunftkausal bedingtes Wesen um. Otfried Höffe: Kants Kritik der reinen Vernunft. Die Grundlegung der modernen Philosophie (München 2003: 254), spricht von einem ,Leitziel', das die dritte Antinomie allerdings nur ,indirekt' thematisiert. Werner Flach: Die vielfältigen Aspekte des Freiheitsbegriffs in der Philosophie Kants (unveröff. Manuskript 2016), hat unlängst zwar versucht, Freiheit bei Kant als Spontankausalität von Intelligibilität zu unterscheiden, muss aber gleichwohl eingestehen, dass Kant die der Spontaneität eigene Unbedingtheit gleich als Intelligibilität charakterisiert. Freilich macht Flach die kosmologische Freiheit nicht eigens thematisch: er legt primär Kants praktische Profilierung aus und unterscheidet dabei das Erkenntnisinteresse, das zivile Interesse, das sittliche Interesse und das ästhetische Interesse. Er setzt dabei eine Auslegung fort, die unlängst dokumentiert ist in Werner Flach: Kant $z u$ Geschichte, Kultur und Recht. Hrsg. von Wolfgang Bock (Berlin 2015). — Kant wird zitiert nach Kants gesammelte Schriften $($ Akademie-Ausgabe $=\mathrm{AA})$, Band, Seite(n); $\mathrm{KrV}$ gemäß der Originalpaginierung. $\mathrm{KrV}=$ Kritik der reinen Vernunft $; \mathrm{KpV}=$ Kritik der praktischen Vernunft; $\mathrm{KU}=$ Kritik der Urteilskraft.
} 
Die Verquickung, um nicht zu sagen: Kontamination der kosmologischen Dimension mit der praktischen Dimension der Freiheit bedarf der Aufhellung. Die skizzierte Konstellation führt systematisch gesehen nämlich zu einer Kantischen Hypothek, die zu tilgen in der kantianisierenden Transzendentalphilosophie noch aussteht, die Hegel jedoch paradigmatisch geleistet hat. Während sich die Kant-Forschung vorwiegend mit Kants Nachweis der Denkbarkeit der Freiheit zufrieden gibt und sodann dessen Beweis(e) für die objektive Realität des Freiheitsbegriffs bzw. dessen Verästelung in Moral, Recht und Tugend nachspürt, hat die Transzendentalphilosophie des südwestdeutschen Neukantianismus bis in die neuere Transzendentalphilosophie von Hans Wagner und Werner Flach hinein aus der Bestimmung von Freiheit als Vermögen eigengesetzlicher Kausalität des tätigen Subjekts ein axiotisches Grundverhältnis gestrickt, das das fundierende Verhältnis für die gesamte Welt des Menschen ausmacht und sich in verschiedene Kultur- oder Geltungssphären spezifiziert Wissenschaft, Moral, Recht, Kunst usw. Hegel problematisiert und kritisiert das kosmologische, von Kant auch transzendental genannte Freiheitsverständnis und qualifiziert es als unzureichend: der Begriff selbst ist ihm das Freie, und der Begriff verursacht nicht etwas anderes, als er selbst ist, sondern manifestiert sich in allem, was irgendwie ist, ist bei sich in seinem Anderen. Hegel nimmt dabei sowohl den kosmologischen Impetus der Freiheit als Ursprung der Erscheinungswelt als auch Kants höchstes Prinzip alles Verstandesgebrauchs, die synthetische Einheit der Apperzeption, radikal ernst. $^{3}$ - Wenn im folgenden Kants Freiheitslehre kritisch betrachtet wird, so ist diese Betrachtung im Übrigen geleitet von der Überzeugung ihrer überragenden Aktualität im Vergleich zu allerlei kursierenden Naturalismen, Utilitarismen, Tugendethiken u. dgl. Hegels philosophische Höhenflüge bilden die Herausforderung, nicht die Niederungen des philosophischen Alltags.

Es gilt daher, Kants Bestimmung kosmologischer oder transzendentaler Freiheit eigens zum Thema zu machen, statt ihn vorschnell als gesichert anzunehmen bzw. praktisch auszuschalten. Ein unbefangener Blick wundert sich vielmehr über die Bestimmungen von Freiheit, die in die kosmologische Antinomie hineinschneien, mögen sie auch gerade von einer modernen, willensorientierten Konzeption von Freiheit durchaus eine prima facie Plausibilität haben: ausgewiesen als Bestimmungen von Freiheit sind sie damit gerade nach Kants kritischen Maßstäben noch lange nicht. Dies ist umso bedeutsamer, als Kants Freiheitskonzeption, wie angedeutet, durchaus praktisch präfiguriert ist. Mit Blick auf das Argumentationsziel und den Argumentationsgang der Kritik der reinen Vernunft mag man dies noch als unwesentlich hinnehmen. Gleichwohl bildet gerade das in der dritten Antinomie erreichte Ergebnis, die Denkbarkeit der Freiheit als Unabhängigkeit von allen bestimmenden Ursachen der phänomenalen Welt und Bestimmbarkeit durch Ursachen der intelligiblen Welt, d.h. die Möglichkeit der Selbstbestimmung statt der durchgängigen Fremdbestimmung, den Ausgangspunkt der kritischen Grundlegung der praktischen Vernunft. ${ }^{4}$ Ohne kosmologische oder transzendentale Freiheit qua Vermögen, eine Reihe von sukzessiven Dingen oder Zuständen in der Welt von selbst anzufangen, gibt es keine praktische Freiheit als Vermögen willentlicher Selbstbestimmung, sondern nur einen unverbrüchlichen Determinismus. Damit aber meldet sich in aller Härte die kritische Forderung nach Rechenschaftslegung transzendentaler Begriffe, noch abgesehen von der Tatsache, dass Freiheit einer der beiden Grundbegriffe der gesamten Kantischen Architektonik der Vernunft ist. Problematisch wird also weniger die Denkbarkeit der Freiheit, sondern vielmehr dass Freiheit ebenjener Sachverhalt ist, als den Kant ihn in seinem transzendentalen oder kosmologischen Freiheitsbegriff in Anspruch nimmt. Dies betrifft

\footnotetext{
${ }^{3}$ Vgl. zu Hegels Lehre von der Freiheit des Begriffs neuerdings Hans Friedrich Fulda: „Der eine Begriff als das Freie und die Manifestationen der Freiheit des Geistes“. In: Hegel - 200 Jahre Wissenschaft der Logik, hg. von Anton Friedrich Koch, Friedrike Schick u. a. (Hamburg 2014: 15-41); Franz Knappik: Im Reich der Freiheit. Hegels Theorie autonomer Vernunft (Berlin 2013); Christian Krijnen: „Freiheit als ursprüngliche Einheit der Vernunft. Hegels begriffslogische Lösung eines Kantischen Problems“. In: Natur und Geist hg. von Wolfgang Neuser und Pirmin Stekeler-Weithofer (Würzburg 2016: 25-52).

${ }^{4}$ - wie Kant selbst Eingangs der Kritik der praktischen Vernunft durch seine Bezugnahme auf die dritte Antinomie unterstreicht $(\mathrm{KpV}, \mathrm{AA} \mathrm{V} \mathrm{15).}$
} 
zunächst die Bestimmtheit der Freiheit, Ursprung der Erscheinungswelt zu sein; sie ermöglicht allererst den praktischen Freiheitsbegriff.

Tatsächlich läßt sich das Profil von Kants kritischer Freiheitskonzeption im Allgemeinen und das der dritten Antinomie im Besonderen aus ihrer Einbettung in der deutschen Schulphilosophie des 18. Jahrhunderts verstehen. ${ }^{5}$ Sogar die makroskopische Andeutungen Kants bezüglich seiner philosophiegeschichtlichen Selbstpositionierung im Spektrum von „Physiokratie“ (KrV, B478), „Epikureism“ und „Platonism“ (KrV, B499) ${ }^{6}$ haben Vorlagen im schulmetaphysischen Bildungsgut der Zeit. ${ }^{7}$ Das soll keineswegs heißen, dass das Profil der Kantischen Freiheitskonzeption keine von ihrer historischen Situierung unabhängige Plausibilität für sich hat, sondern dass es ungemein aufschlußreich ist, Kants transzendentalen oder kosmologischen Freiheitsbegriff im Kontext der damaligen Diskussionslage zu betrachten. Dies betrifft vor allem das Profil der Kantischen Freiheitslehre selbst. Es geraten allerlei Vorentscheidungen und unausgewiesene Konstellationen dieses Profils betreffend in den Blick ${ }^{8}$ - Vorentscheidungen und Konstellationen, die sich gemäß Hegels Analyse auch gar nicht ausweisen lassen, sondern den Übergang zu einem allgemeineren Freiheitsbegriff als dem transzendentalen oder kosmologischen Kants erforderlich machen. Der kosmologische Freiheitsbegriff als Ursprung der Erscheinungswelt ist, bei näherer Betrachtung, vorschnell der Schulmetaphysik entnommen, anstatt ihm transzendental auf den Grund zu gehen. Spontaneität bzw. Freiheit wird entsprechend zu einem schlechterdings fundamentalen Kernthema des nachkantischen Idealismus.

Im Folgenden will ich zunächst Grundzüge der Diskussion der Zeit grob skizzieren, jedenfalls was das für meine These Wichtigste betrifft (2). Sodann kommt Hegels Transformation des Freiheitsbegriffs zur Sprache (3), bevor abschließend am Beispiel Bruno Bauchs das axiotische Grundverhältnis der kantianisierenden Transzendentalphilosophie problematisiert wird (4).

\section{Kant im Kontext der Schulmetaphysik}

Die Debatte über den Spinozismus mag hier als Fluchtpunkt fungieren. Erstens bildet die Auseinandersetzung zwischen der Philosophie Wolffs und dem Hallenser Pietismus zweifelsohne eine der erbittertsten Geistesdispute des 18. Jahrhunderts. Der sachliche Kern des Streits betrifft die Frage: Fatalismus oder Freiheit? Wolff wurde von Lange vorgeworfen, mit einer mechanistischen Welterklärung à la Spinoza (und Leibniz), die Freiheit des Menschen preiszugeben; fatalis necessitas,

\footnotetext{
${ }^{5}$ Vgl. zu Kants Schlüsselbegriffen Spontaneität und Willkür vor allem die glänzende Arbeit von Katsutoshi Kawamura: Spontaneität und Willkür. Der Freiheitsbegriff in Kants Antinomienlehre und seine historischen Wurzeln (Stuttgart-Bad Cannstatt 1996). Vgl. auch: Katsutoshi Kawamura: „Eine begriffsgeschichtliche Skizze der "Willkür" im 18. Jahrhundert. Wolff, Wagner, Feder und Kant“. In: Recht und Frieden in der Philosophie Kants. Akten des X. Internationalen Kant-Kongresses III, hg. von Valerio Rohden, Ricardo Terra u. a. (Berlin 2008: 173-181); Reinhard Finster: ,Spontaneität, Freiheit und unbedingte Kausalität bei Leibniz, Crusius und Kant“. In: Studia Leibnitiana 14 (1982: 266-277); Reinhard Finster: Spontaneität und Freiheit. Eine Untersuchung zu Kants theoretischer Philosophie unter Berücksichtigung von Leibniz, Wolff und Crusius. Univ. Diss. (Trier 1984); Clemens Schwaiger: Alexander Gottlieb Baumgarten. Ein intellektuelles Porträt. Studien zur Metaphysik und Ethik von Kants Leitautor (Stuttgart-Bad Cannstatt 2011).

${ }^{6}$ Vgl. dazu auch unter Berücksichtigung anderer antiker Positionen Heinz Heimsoeth: „Zum kosmotheologischen Ursprung der Kantischen Freiheitsantinomie“. In: Kant-Studien 56 (1966: 206-229).

${ }^{7}$ Vgl. K. Kawamura: Spontaneität und Willkür, a.a.O. [Anm. 5] $124 \mathrm{f}$.

${ }^{8}$ Georg Geismann: „Kant über Freiheit in spekulativer und in praktischer Hinsicht“. In: Kant und kein Ende. Band 1: Studien zur Moral-, Religions- und Geschichtsphilosophie (Würzburg 2009: 119-142), bietet eine wohl dokumentierte und lehrreiche Analyse von Kants Bestimmung transzendentaler und praktischer Freiheit; er stellt allerdings den Entwicklungsgang von Kants Denken über Freiheit nicht in Rechnung. Vielmehr bemüht er sich um die Herstellung einer Kontinuität. Dabei ist schon der praktische Freiheitsbegriff der Kritik der reinen Vernunft unkritisch und entsprechend ohne Nachweis seiner objektiven Realität. Diesen Nachweis liefert Kant erst durch das Faktumtheorem der reinen praktischen Vernunft in der Kritik der praktischen Vernunft, womit er zugleich den transzendentalen Freiheitsbegriff in seiner objektiven Sachhaltigkeit rechtfertigt (und nicht in seiner bloßen Denkbarkeit, wie in der dritten Antinomie der Kritik der reinen Vernunft). Geismann will unbedingt vermeiden, Wasser auf die Mühlen der „Flickwerk-Anhänger“ (ebd. 134) fließen zu lassen. Gleichwohl hat nicht nur Kants Freiheitstheorie innerhalb der kritischen Periode grundlegende Änderungen erfahren, sondern diese Änderungen schreiben zugleich einen Entwicklungsgang fort, der schon früh in Kants vor-kritischem Denken anhob.
} 
alles Geschehen sei unentrinnbar notwendig. ${ }^{9}$ Für die deutsche Aufklärungsphilosophie wurde die Freiheitsfrage durch diesen Disput zu einer Schicksalsfrage, die auch Kants Freiheitsantinomie der Kritik der reinen Vernunft problem- wie quellengeschichtlich - vermittelt besonders über Baumgartens Metaphysik - gespeist hat. Zum zweiten wird in diesem Disput auf ein ,fatalistisches Einheitskonzept reagiert, das gerade Hegel für seinen freiheitsphilosophischen Fortgang über Kant hinaus spekulativ eben als Freiheit umdeutet.

Kants Unterscheidung von transzendentaler oder kosmologischer Freiheit als ,absoluter Spontaneität“ (KrV, B474) und praktischer Freiheit als „freier Willkür“ oder ,arbitrium liberum“ (KrV, B561 f., 830, 836) hat ihre Vorlagen in der Schulmetaphysik. Beide Freiheitsbegriffe sind nicht nur unterschiedlicher historischer und problemgeschichtlicher Herkunft, sondern, was für meine Analyse von großer Bedeutung ist, der transzendentale Freiheitsbegriff selbst speist sich aus zwei unterschiedlichen Problemkontexten. ${ }^{10}$ Dadurch wird nämlich das bei Kant fortwährend wirksame praktische Profil der kosmologischen Freiheit als absoluter oder unbedingter Spontaneität verständlich.

Es betrifft zum einen den ,praktischen' Kontext philosophischer Reflexionen über Wahloder Willkürfreiheit, vor allem deren Stufung in i) ,Spontaneität' als Handlung, ii) ,Willkür` als Handlung nach Belieben und, iii), freie Willkür` als Handlung nach vernünftigem Belieben. Kants transzendentaler Freiheitsbegriff bildet aus dieser Perspektive eine Spezifikation der freien Willkür. Dergleichen Reflexionen über Willkürfreiheit finden sich in der empirischen Seelenlehre der damaligen Schulmetaphysik. Spontaneität und Willkür wurden hierzunächst einfach gleichgesetzt: Wolff übersetzt wirkungsmächtig Leibnizens ,Spontaneität‘ als selbstbestimmtes Handeln mit ,Willkür' und faßt ,Freiheit' als ,vernünftige Willkür' ${ }^{6}{ }^{11}$ bevor Friedrich Wagner und, für Kant wichtiger, Baumgarten Spontaneität und Willkür begrifflich trennen. Im Zuge Wagners unterscheidet Baumgarten drei Stufen der Selbsttätigkeit:

i) spontaneitas, d. h. Handlungen, die aus einem inneren zureichenden Grund erfolgen,

ii) Willkür bzw. arbitrium, d. h. das Vermögen nach eigenem Belieben zu begehren (oder zu verabscheuen),

iii.i) sinnliche Willkür bzw. arbitrium sensitivum als eine von sinnlichem Belieben bestimmte Willkür und

iii.ii) Freiheit bzw. arbitrium liberum als Vermögen, nach vernünftigem Belieben zu begehren (oder zu verabscheuen). ${ }^{12}$

Willkür ist hier also konzeptualisiert als eine besondere Art von Spontaneität und Freiheit wiederum als eine besondere Art der Willkür. Dass Kants transzendentaler Freiheitsbegriff problemgeschichtlich eine Innovation darstellt, verhindert nicht, dass auch die transzendentale Freiheit als eine besondere Art der Willkür gedacht ist, als „unbedingte Willkühr“ (Refl. 3860, AA XVII 316) eben, oder wie Kant in der Kritik der reinen Vernunft sagt, als „absolute Spontaneität“ (KrV, B474). Diese ist nicht bloß, wie die praktische Freiheit, unabhängig von sinnlichen Antrieben, sondern unabhängig von jeglichem äußeren Zwang, nicht von etwas abgeleitet, sondern „unbedingt“

\footnotetext{
${ }^{9}$ Vgl. zum Wolff-Lange-Streit: Albrecht Beutel: „Causa Wolffiana. Die Vertreibung Christian Wolffs aus Preußen 1723 als Kulminationspunkt des theologisch-politischen Konflikts zwischen halleschem Pietismus und Aufklärungsphilosophie“. In: Reflektierte Religion. Beiträge zur Geschichte des Protestantismus (Tübingen 2007: 125-169); Bruno Bianco: „Freiheit gegen Fatalismus. Zu Joachim Langes Kritik an Wolff“" In: Zentren der Aufklärung: I: Halle. Aufklärung und Pietismus, hg. von Norbert Hinske (Berlin 1989: 111-155); K. Kawamura: Spontaneität und Willkür, a.a.O. [Anm. 5] 25 ff., 131 ff.; C. Schwaiger: Baumgarten, a.a.O. [Anm. 5] 79 ff.

${ }^{10}$ Vgl. dazu eingehendK. Kawamura: Spontaneität und Willkür, a.a.O. [Anm. 5].

${ }^{11}$ Christian Wolff: Vernünfftige Gedancken von Gott, der Welt und der Seele des Menschen, auch allen Dingen überhaupt. In: Gesammelte Werke I.2, hg. von Jean École et al. (Hildesheim, New York 1962 ff.), § $519 \mathrm{f}$.

${ }^{12}$ Alexander Gottlieb Baumgarten: Metaphysica. Ed. IV, 1757. In: Kants gesammelte Schriften. Bd. I-XXIX XIV, XV, XVII, hg. von Königlich-Preußische Akademie der Wissenschaften et al. (Berlin 1910 ff.), §§ 712 mit 719.
} 
(Refl. 3860, AA XVII 316), ursprünglich, „originalis“ (Refl. 4548, AA XVII 589), ,absolut“ (KrV, B474).

Zum anderen betrifft der transzendentale Freiheitsbegriff den ,theoretischen“ Kontext philosophischer Reflexionen über die Freiheit oder Selbsttätigkeit des Menschen im Ganzen des Weltgeschehens, also den Kontext der Kosmologie. In dieser Perspektive ist Kants transzendentaler Freiheitsbegriff eine Lösung für das Problem des ,Ursprungs‘ eben jenes Weltgeschehens. Indem Kant in seiner 1755er Habilitation Freiheit im kosmologischen Verstande zunächst in den Bahnen Wolffs denkt und entsprechend als Spontaneität aus einem inneren Grund, ${ }^{13}$ der selbst in der Reihe vorhergehender Gründe liegt, ist sein Denken über Freiheit von Anfang an mit dem Problem des Verhältnisses vom Satz des zureichenden oder bestimmenden Grundes zur Freiheit qua Entstehen ohne vorhergehenden Grund, also als schlechthin erster Ursache, befrachtet. Es wird ihm seit den 60er Jahren ausdrücklich zum Problem. Kants Lösung läuft bekanntlich auf die Unterscheidung von sensibler und intelligibler Welt hinaus: der Satz vom zureichenden Grund gilt als „Grund möglicher Erfahrung, nämlich der objectiven Erkenntniß der Erscheinungen in Ansehung des Verhältnisses derselben in Reihenfolge der Zeit“ (KrV, B246). Für die kosmologisch verstandene Freiheit gilt indes, dass sie als absolute Spontaneität oder transzendentale Freiheit keinen solchen vorhergehenden bestimmenden Grund hat, sondern davon unabhängig und folglich intelligibler Art ist.

Diese kosmologische Profilierung des Freiheitsproblems hängt eng mit dem Problem menschlicher Freiheit zusammen. Sie entzündet sich nämlich an der Wolff-Lange-Kontroverse, also am Fatalismusstreit. Schon Wolff denkt das Weltgeschehen nicht als gegründet in einer sich selbst verursachenden und notwendigen unendlichen Rückgangsreihe, sondern in einer mit Gott als schlechthin erster, extramundaner und frei wählender Ursache oder Kausalität abgeschlossenen und als Ganzes zufälligen Reihe. ${ }^{14}$ Gott ist der Urheber, das Unbedingte, hat das Vermögen, einen Zustand des Weltlaufs (bzw. diesen als solchen) von selbst anzufangen. Die schulphilosophischen Debatten in puncto kosmologischer Freiheit kreisen sodann um die Frage nach der Möglichkeit oder Unmöglichkeit, einen Rückgang ins Unendliche zu denken, sodass die praktische oder psychologische Dimension der Freiheit in den Hintergrund rückt. Gerade Baumgarten hat das Problem des unendlichen Rückgangs eingehend in seiner Metaphysik thematisiert. ${ }^{15}$ Das ens extramundanum als wirkende Ursache außerhalb der Welt ist unbedingt und notwendig, die Welt bedingt und zufällig; die Rückgangsreihe ist nicht unendlich, sondern hat ihren Grund außer sich, während diesem Grund das Vermögen eignet, einen Zustand des Weltlaufs überhaupt erst anzufangen. Die Welt ist daher nicht gedacht, wie bei Spinoza, als die Selbstentfaltung einer unendlichen Substanz. Gleichwohl bleibt undurchsichtig, wie Freiheit als Spontaneität und der Satz des zureichenden Grundes sich versöhnen lassen, d. h. wie die Freiheit des Menschen denkbar wird. Kants dritte Antinomie bietet eine kosmologische Lösung für dieses praktische Problem. An die Stelle des Schemas ens extramundanum - mundus bzw. ,Unbedingtes: Gott - Bedingtes: Mensch` tritt die Vernunft im Menschen. Kosmologische Freiheit ist entsprechend absolute Spontaneität.

Damit bildet der transzendentale Freiheitsbegriff zugleich auch, was Kant öfters betont und systematisch gänzlich schlüssig ist, den Boden für den praktischen Freiheitsbegriff. Hier wie dort ist der Satz vom zureichenden oder bestimmenden Grund maßgebend: dass und wie etwas ist, hat seine Ursache oder seinen Grund. Während die ontotheologischen schulmetaphysischen Vorgänger Kants das Problem von Spontaneität und vorhergehendem Grund durch den Rekurs auf ein ens extramundanum, d. h. Gott als sich selbst bestimmendes Wesen, versuchten zu lösen, erweist sich für

${ }^{13}$ Wolff faßt Spontaneität im Sinne von Leibniz. Unter Bezug auf Aristoteles heißt es bei Leibniz, eine Handlung ist spontan, wenn ihr Prinzip in dem Handelnden liegt (Gottfried Wilhelm Leibniz: Essais de théodicéesur la bonté de dieu, la liberté de l'homme et l'origine du mal. In: Die philosophischen Schriften von Gottfried Wilhelm Leibniz VI, hg. von Carl Immanuel Gerhardt (Hildesheim 1978), 296 (§ 301).

${ }^{14}$ C. Wolff: Deutsche Metaphysik, a.a.O. [Anm. 11] §§ 1080 mit 579.

${ }^{15}$ A. G. Baumgarten: Metaphysica, a.a.O. [Anm. 12] $\S 380 \mathrm{f}$. 
Kant die Vernunft selbst als das Unbedingte und damit als transzendentale Freiheit, absolute Spontaneität. Freiheit als absolute Spontaneität ist das Vermögen, „einen Zustand von selbst anzufangen“ (KrV, B561, vgl. 474), also ein allgemeinerer Sachverhalt als „,praktische“ Freiheit qua nicht-sinnliche Willkür, d. h. als eine Willkür, die „unabhängig von sinnlichen Antrieben, mithin durch Bewegursachen, welche nur von der Vernunft vorgestellt werden“, bestimmt werden kann (arbitrium liberum) (KrV, B830).

Die Unterscheidung von Spontaneität und Willkür ergibt sich dabei erst im Laufe der 60er Jahre. Freiheit ist für Kant zunächst auf Vernunft gegründete Willkür, wobei er sich an Baumgartens Einteilung der Selbsttätigkeit anlehnt (spontaneitas, arbitrium, liberum arbitrium) und Freiheit als arbitrium faßt. Dabei unterliegen freie Handlungen dem Satz vom Grunde, letztlich Gott. Freiheit als Unabhängigkeit von einem vorhergehend bestimmenden Grund kennt Kant vorerst noch nicht, sieht dann jedoch, dass Freiheit als Willkür bzw. unbedingte Willkür eine spontaneitas simpliciter talis, also eine schlechthin erste, unbedingte Spontaneität voraussetzt, sodass sich das Problem ergibt, wie eine solche unbedingte Willkür innerhalb der Reihe bestimmender Gründe auftreten kann (zumal wenn der Mensch das Geschöpf eines göttlichen Urhebers ist). In den 70er Jahren gelangt Kant dann zur terminologischen Unterscheidung von transzendentaler und praktischer Freiheit (arbitrium liberum), die Kant beide als Willkürfreiheit bezeichnet: Transzendentale Freiheit bezieht sich entsprechend auf jene Handlungen der Willkür, die „schlechthin spontan“ oder „ursprünglich“ sind (Refl. 4547, AA XVII 589), unabhängig von jeglicher äußeren Nötigung. Freiheit wird hier allerdings noch nicht in Spontaneität und Willkür unterschieden. In den späten 70er Jahren kommt es dann zu einer schärferen Unterscheidung von praktischer und transzendentaler Freiheit dahingehend, dass er die praktische Freiheit zur empirischen, die transzendentale hingegen zur rationalen Psychologie zählt. Diese thematisiert die Willkür nicht als Vermögen, sich nach (sinnlichen und vernünftigen) Gründen zu bestimmen, sondern als unbedingte oder absolute Willkür und damit als schlechthin erste Spontaneität oder unbedingte Ursache. Während der Rekurs auf ein principium externum, einen Urheber menschlicher Spontaneität, diese zu einer spontaneitas automatica macht, also zur „Freiheit eines Bratenwenders" etwa, werden durch die spontaneitas absoluta menschliche Handlungen als frei begreiflich. Es kommt also zur Differenzierung von Freiheit als Spontaneität und Willkür.

In der Kritik der reinen Vernunft wird diese Differenz kosmologisch verwertet. Hier behandelt Kant das Problem der Freiheit als absoluter Spontaneität kosmologisch. Er greift dabei schulmetaphysische Diskussionen auf, die sich anläßlich des Wolff-Lange-Streits ergaben, und zwar besonders Überlegungen in der Kosmologie der Baumgartenschen Metaphysik zur Unmöglichkeit eines infiniten Regresses der bestimmenden Ursachen oder Seinsgründen; dergleichen läuft Baumgarten zufolge auf eine Reihe zufälliger Dinge hinaus, ohne erste Ursache, von der ganzen Reihe so wenig wie von irgendeinem Teil. ${ }^{16}$ Das Denkmuster des kosmologischen Gottesbeweises fortschreibend, führt die Existenz zufälliger Wesen in der Welt auf die eines notwendigen Wesens als erster wirkender Ursache außerhalb der Welt. ${ }^{17}$ Wie bei Leibniz, Wolff $u$. a. ist diese erste Ursache ein notwendiges, unendliches und unveränderliches ens extramundanum, näherhin: Gott, der Urheber der zufälligen, endlichen, veränderlichen Welt. Hält man indes einen Fortgang ins Unendliche für notwendig, dann ist zwar der Rekurs auf ein Extramundanes überflüssig, zugleich aber auch eine freie Willkür dahin.

Ebendeshalb wurde der Spinozismus, das philosophische Schreckgespenst des 18. Jahrhunderts, so vehement bekämpft: ihm zufolge ist die Welt nicht zufällig, sondern notwendig, und damit auch jedes innerweltliche Geschehen. Für menschliche Freiheit wie einen außerweltlichen Gott gebe es keinen Platz. Spinozismus heißt in dieser Deutung dann soviel wie Fatalismus. Die Welt existiert notwendig, nicht zufällig, besteht ewig, nicht zeitlich, und außerhalb von ihr existiert nichts:

${ }^{16}$ ebd. $\S 380$.

${ }^{17}$ ebd. $\$ 381$. 
sie ist selbst unendlich, ihre eigene wirkende Ursache (causa sui). Schon für Wolff erweist sich nicht nur die Annahme eines extramundanen Urhebers vonnöten, sondern dieser ist zugleich als freie Ursache von allem konzipiert, die Welt Ergebnis seiner freien Wahl (des Besten, d. h. der besten aller Welten). ${ }^{18}$ Kants kosmologische oder transzendentale Freiheit ist nach diesem Denkmuster einer ersten wirkenden Ursache, die einen Zustand bzw. ersten Zustand des Weltgeschehens von selbst anfängt (KrV, B561), gestrickt. ${ }^{19}$ Er konzipiert die Welt nicht, wie Baumgarten mit Blick auf Spinoza schreibt, als unendliche Substanz, sondern eine solche Substanz als ein ens extramundanum. ${ }^{20}$

Kant, mit Baumgartens Metaphysik bestens vertraut, kam erst allmählich zu einem Konzept kosmologischer Freiheit, also zur Erkenntnis, wie es in einer Reflexion aus den 60er Jahre schon heißt, dass „das oberste Principium (der Möglichkeit) des entstehens“ die Freiheit ist (Refl. 4033, AA XVII 392). Diese erste Ursache außerhalb der Reihe der Erscheinungen findet sich in den „intellektualen“ Reihen, in der „Vernunft“; wo Vernunft, da auch ein Erstes: „Freyheit und oberstes Wesen“ (Refl. 5362, AA XVIII 162). Die „Ursache der Erscheinung ist nicht in der Erscheinung enthalten“ (Refl. 5369, AA XVIII 163). Es gibt also ein Erstes, und somit keine bloß spinozistische Unendlichkeit: transzendentale Freiheit bzw. absolute Spontaneität in der Vernunft des Menschen, folglich nicht bloß in Gott als schlechterdings erster wirkender Ursache. Das Wesen kosmologischer Freiheit als absoluter Spontaneität ist es, schlechthin erste Ursache zu sein. Freiheit ist der Ursprung der Erscheinungswelt - freilich als willkürtheoretisch konzipierte Kausalität.

Kant denkt sie als transzendentale Freiheit, die absolute Spontaneität ist: „libertas transcendentalis“, „unbedingte spontaneitaet der Handlung“ (Refl. 4759, AA XII 710). Sie bildet den ersten Grund bzw. die schlechthin erste Ursache der Reihe der Erscheinungen. „Kausalität nach Naturgesetzen“ reicht somit nicht aus, um Erscheinungen zu erklären; zusätzlich ist eine „Kausalität durch Freiheit" (KrV, B472) gefordert. Es gibt für Kant eine extramundane, unbedingte, erste Ursache der Natur, wie von Wolff cum suis behauptet: die Natur ist nicht selbst das unbedingte und notwendige Wesen, wie im Gegenbild dieser These, dem Spinozismus. Gleichwohl ist die Freiheitsproblematik für Kant trotz des dem Freiheitsbegriff nunmehr angewachsenen kosmologischen Profils immer verbunden mit einem praktischen Interesse, das ihn von Anfang an umgetrieben und das er bis in die 70er Jahre hinein im Kontext der Auseinandersetzung mit der empirischen Psychologie der Baumgartschen Metaphysik erkundet hat. ${ }^{21}$ Der transzendentale Freiheitsbegriff bildet für ihn geradezu die Grundlage des praktischen Freiheitsbegriffs, und ebenjener macht das „eigentliche Moment der Schwierigkeit“ aus (KrV, B561). Die Zurechnung einer Handlung setzt eine unbedingte Willkür voraus bzw. eine ,ursprüngliche Handlung, wodurch etwas geschieht, was vorher nicht war“ (KrV, B572), und damit kosmologische Freiheit. Deren Möglichkeit wird mit Kants transzendentalem Idealismus allererst plausibel: die sensible Welt gründet in der intelligiblen; Erscheinungen sind, was sie sind, nur gemäß den Bedingungen der Vernunft.

\section{Hegel spekulative Transformation transzendentaler Freiheit}

Zwar deontologisiert Kant die Erste Ursache, die in der Schulmetaphysik Gott ist, zugunsten eines transzendentalen Idealismus der Vernunft im Menschen - er hält jedoch am praktischen Profil der Freiheitsbestimmung fest, d. h. er schreibt die tradierte Auffassung, Freiheit sei ein willkürtheoretisch

${ }^{18}$ C. Wolff: Deutsche Metaphysik, a.a.O. [Anm. 11] §§ 1080 f., 1083.

${ }^{19} \mathrm{Zu}$ diesem Schluß kommt K. Kawamura: Spontaneität und Willkür, a.a.O. [Anm. 5] 133.

${ }^{20}$ A. G. Baumgarten: Metaphysica, a.a.O. [Anm. 12] § 388.

${ }^{21}$ Noch 1798 heißt es in einem Brief an Garve über den Anlaß der Kritik der reinen Vernunft und die Freiheitsantinomie: „Nicht die Untersuchung vom Dasein Gottes, der Unsterblichkeit etc. ist der Punkt gewesen von dem ich ausgegangen bin, sondern die Antinomie der r.V.: »Die Welt hat einen Anfang -: sie hat keinen Anfang etc. bis zur vierten: Es ist Freiheit im Menschen, gegen den: es ist keine Freiheit, sondern alles ist in ihm Naturnothwendigkeit«; diese war es welche mich aus dem dogmatischen Schlummer zuerst aufweckte und zur Kritik der Vernunft selbst hintrieb, um das Skandal des scheinbaren Widerspruchs der Vernunft mit ihr selbst zu heben.“(AA XII 257 f.) 
verfaßtes Vermögen der Kausalität eines Urhebers, gar eines extramundanen Urhebers der Erscheinungswelt fort. Entsprechend ist der transzendentale Grund von naturgesetzlich Bedingtem als eine ,Folgen bewirkende Ursache` gedacht, näherhin als Willkürvermögen, als Vermögen, von selbst so oder anders handeln zu können, also aus Möglichkeiten zu wählen und sie durch Handlungen zu verwirklichen. Als Spontankausalität bewirkt sie Erscheinungen, die Kant zufolge ungeachtet der Vernünftigkeit (Vernunftbedingtheit) dieses Bewirkens hätten anders sein können, also als Wirkungen von Spontankausalität kontingenterweise so sind, wie sie sind. ${ }^{22}$ Die Beziehung von Ursache und Wirkung ist insofern äußerlicher Natur, d. h. dualistisch konzipiert, auch wenn Kant eine jedwede wirkende Ursache ihrem Charakter nach als ,gesetzlich“ bestimmt denkt. ${ }^{23}$

Die Ursprungseinheit läßt sich daher nolens volens nicht mehr als Freiheit konzipieren. Freiheit beträfe allenfalls die formale Bestimmtheit des Verhältnisses, nicht auch dessen inhaltliche Bestimmtheit. Das kosmologische Verhältnis von Erscheinungswelt und deren Grund oder Ursache ist keine der Naturgesetzlichkeit analoge Kausalität aus Freiheit; Kants Spontankausalität kann daher nicht die gesuchte Ursache der Erscheinungswelt sein. Das Kausalverhältnis ist so oder so kein absolutes Verhältnis, d.h. die so konzipierte kosmologische Einheit von Grund und Folge kein intrinsisches, sondern ein äußerliches Gefüge und deshalb nur denkbar unter Voraussetzung ursprünglicher, allerdings noch ungedachter Voraussetzungen.

Hegel thematisiert sie eigens am Ende seiner Wesenslogik und damit im Rahmen kosmologischer Konstellationen, die sich sodann als solche des „Begriffs' erweisen: der gesuchte kosmologische Grund entpuppt sich als der (nicht-kosmologisch verfaßte, sondern jedweder Kosmologie noch zugrundeliegende) ,Begriff“. Das „Wesen“ erweist sich in Hegels Konzeption näherhin als „Grund“ der „Existenz“ bzw. der „Erscheinung“ bzw. der „Wirklichkeit“ (E §§112-159; II Zweites Buch); der Grund ist geradezu das Wesen als „Totalität“ (E §121) gesetzt. Das Grundverhältnis (von Grund und Begründetem) als inneres, „absolutes Verhältnis“ ergibt sich bei Hegel sodann aus dem kosmologischen Format des Substantialitätsverhältnisses, des Kausalitätsverhältnisses und der Wechselwirkung als Selbstverhältnis des Begriffs. Der Begriff wiederum qualifiziert sich durch seine Momente des Allgemeinen, Besonderen und Einzelnen, wodurch der Rekurs auf extern Vorgegebenes bzw. Freiheit, die bloße Spontaneität und damit Willkür bzw. Zufall ist, in den Begriff zurückgenommen wird. Der so verstandene Begriff ist für Hegel das Freie.

Mit dem Übergang vom Abschnitt ,Die Wirklichkeit‘ zur ,Lehre vom Begriff` deduziert Hegel in seiner Logik den Begriff des Begriffs und die ihm eigene Freiheit. „Der Begriff ist das Freie“ (E §160), Freiheit die ,absolute Negativität des Begriffs als Identität mit sich“ (E §382). Hegel kommt zu dieser Erkenntnis, indem er Kants kosmologischen Freiheitsbegriff und Spinozas Substanzbegriff aus ihrem gemeinsamen Grund begreift, dem Begriff als dem Freien eben. Freiheit ist ihm als solche kein Vermögen der Kausalität mehr - für Hegel eine Verstandesauffassung von Freiheit $-{ }^{24}$ sondern Bei-sich-selbst-sein-im-Anderen. Obwohl für Hegel die vielgescholtene

${ }^{22} \mathrm{Vgl}$. auch KrV, B584 f über das Anders-sein-Können der Erscheinungen. Diese Thematik führt zum viel verhandelten Formalismus-Problem in Kants Philosophie. Vgl. dazu neuerdings meine Studien "Sittlichkeit and the Actuality of Freedom", in: The Palgrave Hegel Handbook, ed. M. Bykova and K. Westphal, Houndmills, Basingstoke, Hampshire (im Erscheinen), sowie „Die Wirklichkeit der Freiheit begreifen: Hegels Begriff von Sittlichkeit als Voraussetzung der Sittlichkeitskonzeption Kants“, in: Folia Philosophica (im Erscheinen). Hegels Begriffslehre soll die Abstraktheit überwinden, die bei Kant im Verhältnis zwischen Allgemeinem und Besonderem herrscht. (Begriffliche) Notwendigkeit (im Zusammenhang von Allgemeinem, Besonderem und Einzelnem) tritt hier entsprechend an die Stelle von Zufälligkeit.

${ }^{23}$ Wie jede „wirkende Ursache“, fasst Kant auch den „Charakter“ einer durch Freiheit wirkenden Ursache als „Gesetz“ (KrV, B567), sodass einem „Subjecte der Sinnenwelt“ dadurch ein „empirischer Charakter“ wie ein „intelligibler Charakter“ zukommt; seine „Handlungen“ sind folglich zum einen als ,Erscheinungen“ naturgesetzlich bedingt, zum anderen aber ist das Subjekt auch „Ursache jener Handlungen als Erscheinungen“ (KrV, B567).

${ }^{24}$ Freiheit als ,Vermögen“ (Freiheit als Möglichkeit), das auf einen so oder so gegebenen Stoff ,angewandt“ wird, ist Hegel eine bloße,Verstandesauffassung' dessen, was Freiheit ist (R $\S 10 \mathrm{~A}$, vgl. auch $\S 15 \mathrm{~A})$. Hegel wird wie folgt zitiert: I = Wissenschaft der Logik. Erster Teil. Hg. von Georg Lasson (Leipzig 1951); II = Wissenschaft der Logik. Zweiter Teil. Hg. von Georg Lasson (Leipzig 1951); E = Enzyklopädie der philosophischen Wissenschaften im Grundrisse (1830). Hg. von Friedhelm Nicolin und Otto 
Substanzphilosophie Spinozas ein Gegenmodell zur Konzeption einer umfassenden Freiheit als spontankausaler Grundlage der Erscheinungswelt bietet, bedarf sie, wie Hegels berühmte Formel von der Subjektwerdung der Substanz (vgl. PG 18 mit II 219) impliziert, einer subjektivitätstheoretischen Aneignung und Transformation, - jedenfalls wenn sie durch Kants transzendentale Philosophie hindurch und nicht an ihr vorbei bewerkstelligt werden soll. ${ }^{25}$ Im Zuge dessen ergeben sich die „absolute Macht“ und die „blinde Notwendigkeit“, die Spinozas Substanz eignen, als die Freiheit, die das Denken, der Begriff oder die Idee ist. Die kosmologische Einheit erweist sich demzufolge als immanente Notwendigkeit des begreifenden Denkens. Wie Hegel sagt, legt das Absolute „sich selbst aus“, sodass sich die Bestimmungen des Absoluten als „,identisches Setzen seiner selbst“ ergeben (II 185, vgl. 157 f., 163 f.). Diesen Manifestations- oder Selbstauslegungsprozess des Absoluten expliziert Hegel dergestalt, dass das wahrhaft absolute Verhältnis die ,gesetzte Einheit seiner in seinen Bestimmungen“ ist, d. h. der „Begriff“ (II 185, 213 ff.). Die erreichte selbstbezügliche Einheit der absoluten Substanz ist der Begriff in seiner Allgemeinheit: sich manifestierende Selbstbeziehung - Freiheit. Damit ist diejenige Freiheit, die der Begriff ist, der Grund der Wirklichkeit als Erscheinung. Der Grund der Wirklichkeit, der, mit Spinoza, zunächst Substanz schien, stellt sich heraus als jene „Freiheit, welche die Identität des Begriffs ist“ (II 218). Im Begriff „eröffnet“ sich, wie Hegel sagt, „,das Reich der Freiheit“ (II 218 f.).

Hegel denkt das kosmologische Verhältnis von Ursache und Wirkung folglich nicht mehr, wie Kant, als eine der Naturgesetzlichkeit analoge ,Gesetzlichkeit‘ (gesetzliche Notwendigkeit) aus Freiheit. Gesetzliche Notwendigkeit ist kein explicans von Freiheit mehr. Der kosmologische Grund ist weder bloße (den Einzelfall bestimmende) Notwendigkeit des Gesetzes noch gesetzlose Zufallsspontaneität. Freiheit ist nach wie vor spontane Tätigkeit. Sie ist jedoch nicht mehr durch Bestimmungen kausaler Verhältnisse wie Notwendigkeit, Zufälligkeit, Gesetz, Ursache, Wirkung charakterisiert, sondern durch die Struktur des ,spekulativen Begriffs': Allgemeines, Besonderes, Einzelnes. Das Freie, das der eine Begriff ist, bleibt als Allgemeines, Besonderes und Einzelnes in seiner Tätigkeit durchgängig ,bei sich' und ist zugleich als ,in sich' differenzierte Einheit im anderen seiner Momente. Solches Beisichselbstsein, sich ,von sich`aus, also ,spontan`, ,zu sich` bestimmen, ist wahrhafte ,Selbstbestimmung'. Sie ist zugleich Vermittlung seiner Momente des Freien miteinander und sich selbst, wahrhafte ,Selbstvermittlung', und insofern immer noch Vermittlung unter eigenem (begrifflichem) Gesetz, ,Autonomie‘. Entsprechend ist das Allgemeine nicht einfach ein wie auch immer gegebenes Gesetz (Autonomie, Heteronomie), welches das Einzelne zu befolgen bemüht ist, sondern durchgängige ,Selbstbestimmung‘ des Allgemeinen zum Einzelnen; das Einzelne wiederum ist nicht einfachhin dem Allgemeinen unterworfen, sondern es bestimmt sich innerhalb des Allgemeinen zum Allgemeinen. Solche Freiheit spontaner Selbstbestimmung und vollständiger Selbstvermittlung als die Tätigkeitsweise des Begriffs und der ihm eigenen Notwendigkeit ist grundlegend für jedwede Spezifikation von Freiheit (namentlich geistiger Freiheit). Als ,absolute Negativität des Begriffs als Identität mit sich“ (E §382) erhält sie sich bei Hegel in allem Fortgang, sowohl innerhalb der logischen Entwicklung des Begriffs als auch des Realen.

Philosophiehistorisch gesehen, hebt Hegel im Rahmen dieser Überlegungen nicht nur den Rationalismus und Empirismus (II 184 ff.) in die Freiheit des Begriffs auf, sondern schließlich gar noch Kants transzendentale Einheit der Apperzeption (II 221 ff.). Dies ist schon deshalb ein interessanter Gesichtspunkt, weil Kants kosmologische Grundlage als Spontaneität selbst der Ausfluß jener ursprünglicheren Spontaneitätskonstellation ist. Kant hat die synthetische Einheit der Apperzeption geradezu als den höchsten Punkt des Verstandes qua Vermögen der Spontaneität (der

Pöggeler (Hamburg 1991); R = Grundlinien der Philosophie des Rechts. Hg. von Johannes Hoffmeister (Hamburg 1955). PG = Phänomenologie des Geistes. Hrsg. von Wolfgang Bonsiepen und Reinhard Heede (Hamburg 1980).

${ }^{25}$ Vgl. für eine Deutung Christian Krijnen: „Freiheit als ursprüngliche Einheit der Vernunft. Hegels begriffslogische Lösung eines Kantischen Problems“. In: Natur und Geist, hg. von Wolfgang Neuser und Pirmin Stekeler-Weithofer (Würzburg 2016: 25-52). 
Begriffe, Erkenntnisse) bzw. der Transzendentalphilosophie bezeichnet, ${ }^{26}$ d. h. als das schlechthinnige Ursprungsprinzip von Objektivität oder Gegenständlichkeit.

Nicht umsonst beginnt Hegel nach seinem wesenslogischen Durchlauf durch den Rationalismus und Empirismus die anschließende ,Begriffslogik“ mit Ausführungen zum „Begriff im Allgemeinen“, in denen ausgerechnet zur Sprache kommt, was nach Hegelscher Auffassung zu den „tiefsten und richtigsten Einsichten“ von Kants Kritik der reinen Vernunft gehört: die „ursprünglichsynthetische Einheit der Apperzeption“ (II 221). Entgegen eines bloß ,,äußerlichen Verhältnisses“, habe Kant die „Einheit, die das Wesen des Begriffs“ ausmacht, als ursprünglich synthetische Einheit der Apperzeption, des „Ich denke oder des Selbstbewußtseins“ gedacht. Kant überwinde somit die Äußerlichkeit von Ich und Verstand bzw. Begriff und Gegenstand. Zu Recht betont Hegel, dass nach Kantischer Auffassung das „Object“ das ist, „in dessen Begriff das Mannigfaltige einer gegebenen Anschauung vereinigt ist“ (KrV, B137). Alle „Vereinigung“ von Vorstellungen ist „Einheit des Bewußtseins in der Synthesis derselben“ (KrV, B137); es ist also die „Einheit des Begriffs“, durch die etwas „Objekt“ ist (II 222). Die Bestimmung des Begriffs als des wahrhaft ,absoluten Verhältnisses“ führt so gesehen die Elemente der Kantischen Relationskategorien, die traditionellerweise jenes Verhältnis qualifizieren, zurück in den „Grundsatz der synthetischen Einheit der Apperception“ als „oberstes Princip alles Verstandesgebrauchs“ (KrV, B §17).

Freilich hält Hegel Kants Rückführung in die Einheit des Selbstbewußtseins als absolute Grundlage für unzureichend, weil das Verhältnis des Begriffs zur „Realität“ qua „Objektivität“ dem Begriff als „Subjektivität“ doch „,gegenübergestellt“ (II 223, vgl. 223 ff.), das Verhältnis von Anschauung und Begriff, Verstand und Sinnlichkeit, Form und Inhalt als ein bloß abstraktes Verhältnis begriffen wird (,Formalismus'). Es fehlt bei Kant somit das Hegelisch verstandene „Prinzip der Bestimmung“ (E § 508), d. h. die jedwede Äußerlichkeit aufhebende Realisierung des Begriffs durch seine Momente des Allgemeinen, Besonderen und Einzelnen. Insofern vollendet Hegels Begriffslehre, dasjenige, was er als Grund für die Schwere der Kantischen transzendentalen Deduktion ausgibt: dass über die bloße „Vorstellung“ des Verhältnisses von „Verstand“ und „Ich“ bzw. „Begriff“ zu einem „Ding und seinen Eigenschaften und Akzidenzien“ zum „Gedanken“ fortgegangen wird (II 221). Der spekulativ verstandene Begriff hat sich als diese gesuchte absolute Grundlage ergeben (vgl. auch II 220). Hegel kommt somit zu einem grundsätzlich anderen Freiheitsverständnis als Kant, indem er den willkür- und kausaltheoretischen Freiheitsbegriff überformt und zu einem nachgeordneten Begriff, der Geistphilosophie nämlich, zurückstuft. Dabei nimmt er gerade Spinoza als All-Einheitsdenker ernst und bereichert die Fatalismus-Debatte durch einen neuen und grundlegenden Gesichtspunkt.

4. Das axiotische Grundverhältnis der kantianisierendern Transzendentalphilosophie als kausaltheoretische Normativitätskonzeption

Mit seiner Kritik und Überwindung der transzendentalen Freiheitskonzeption Kants kritisiert und überwindet Hegel, systematisch gesehen, jedoch zugleich eine Auffassung, die in dieser oder jener Akzentuierung auch für die kantianisierende Transzendentalphilosophie maßgebend geworden und geblieben ist: Freiheit als Vermögen eigengesetzlicher Kausalität des tätigen Subjekts. Gerade die südwestdeutschen Neukantianer haben in ihrer bis heute leider grob unterschätzten Wertphilosophie den Finger darauf gelegt, dass Normierungsverhältnisse das fundierende Verhältnis für die gesamte Welt des Menschen ausmachen: die verschiedenen Kultur- oder Geltungssphären - Wissenschaft, Moral, Recht, Kunst, Religion usw. - sind allesamt Spezifikationen eines axiotischen

${ }^{26}$ „Und so ist die synthetische Einheit der Apperception der höchste Punkt, an dem man allen Verstandesgebrauch, selbst die ganze Logik und nach ihr die Transscendental-Philosophie heften muß, ja dieses Vermögen ist der Verstand selbst.“ (KrV, B134 Anm., vgl. $\mathrm{KrV}, \mathrm{B} \S 17)$ 
Grundverhältnisses. ${ }^{27}$ Es ist dies ein Bedingungsverhältnis von Wert (Orientierungsdeterminante, Geltungsgesetz), wertendem Subjekt und Kulturgut. Bei dieser wertgemäßen ,Selbstgestaltung ' des Subjekts, das darin Kultur schafft, handelt es sich letztlich um eine Selbstgestaltung gemäß Werten, die mit seiner Subjektität und damit seiner Freiheit selbst verbunden sind: um autonome Werte oder Eigenwerte. Der Begriff der Selbstgestaltung betrifft also die Wertbestimmtheit ,überhaupt ` des Subjekts. Im axiotischen Grundverhältnis wird aus geltungsnoetischer Sicht, d. h. aus der Perspektive des Subjekts, das Subjekt durch den Begriff der Selbstgestaltung grundlegend bestimmt. Freiheit ist somit eine geltungsnoetische Qualifikation, eine Qualifikation des Subjekts. Dieses axiotische Grundverhältnis und die ihm eigene Idee der Selbstgestaltung des Subjekts sind wegweisend für die spätere Transzendentalphilosophie Wagners oder Flachs etwa. Wagner macht dabei wenigstens noch den Versuch, Freiheit auch als umfassende Qualifikation zu denken und sie entsprechend als eine der Grundcharaktere des Subjekts aufzufassen, und zwar: in Kantischer Weise, als „Selbstbestimmung“ des Subjekts, als Selbstbestimmung sowohl gemäß dem eigenen Entwurf als auch als Selbstbestimmung ebendieses Entwurfs selbst ${ }^{28}$ Freiheit als Qualifikation des Subjekts wird dabei näherhin und erneut Kantisch als Thema der „Selbstgestaltung“ des Willens im Rahmen einer „Ethik“ ausgearbeitet. ${ }^{29}$ Flach hingegen faßt Freiheit von vornherein bloß als solches Bestimmungsstück des „Wollens" auf und behandelt sie, allerdings nicht als konstitutives, sondern als universal-regulatives Prinzip, im Kontext der „Lehre vom sittlichen Subjekt““ ${ }^{30}$ Bei beiden Transzendentalphilosophen jedoch liegt dieser Thematisierung das axiotische Grundverhältnis zugrunde. ${ }^{31}$ Die Welt des Menschen, der Inbegriff der verschiedenen Kultur- oder Geltungssphären, erweist sich als Inbegriff je spezifischer Ausprägungen des axiotischen Grundverhältnisses. Immer ist sie durch die Struktur eines Werte qua Geltungsgesetzlichkeiten anerkennenden (wertbezogenen) und darin Kulturgüter schaffenden Subjekts prinzipiiert. Die willkür-kausaltheoretische Modellierung bleibt also insofern strukturell erhalten, als das Subjekt sich selbst gemäß der ihm eigenen Normativität oder Wertgesetzlichkeit gestaltet, sich darin so oder so, in geltungsdifferenter Weise, zu einem Wert verhält, und damit Welt schafft. ${ }^{32}$

Im Folgenden und abschließend will ich jedoch nicht gegenwärtige Konzeptionen der Transzendentalphilosophie näher thematisieren, sondern den Versuch eines der scharfsinnigsten Neukantianer und besten ihrer Kant-Interpreten, Bruno Bauchs. Bauch hat nämlich versucht, das Verhältnis von Natur und Freiheit in einer Auseinandersetzung mit Kant zu klären. Die Probleme, die sich dabei hinsichtlich seiner eigenen Konzeption ergeben, mögen als exemplarisch für das Einheitsdenken im Rahmen des axiotischen Grundverhältnisses gelten. Diese Kantische Erblast wiegt also bis heute schwer. Unbeschadet dessen kommt dem axiotischen Grundverhältnis der kantianisierenden Transzendentalphilosophie eine außerordentliche Bedeutsamkeit zu; es hat, soweit ich sehe, nur in Hegels Ideenlehre einen wirklich ernsthaften Konkurrenten.

\footnotetext{
${ }^{27}$ Vgl. dazu Christian Krijnen: Nachmetaphysischer Sinn. Eine problemgeschichtliche und systematische Studie zu den Prinzipien der Wertphilosophie Heinrich Rickerts (Würzburg 2001: Kap. 2.3, 6.3, 7.2 f.); Christian Krijnen: Philosophie als System. Prinzipientheoretische Untersuchungen zum Systemgedanken bei Hegel, im Neukantianismus und in der Gegenwartsphilosophie (Würzburg 2008: Kap. 4.2.2; 5.4); Christian Krijnen: „Das Dasein der Freiheit. Geltungsrealisierung bei Hegel und in der kantianisierenden Transzendentalphilosophie“. In: Kulturphilosophie. Probleme und Perspektiven des Neukantianismus, hg. von Christian Krijnen, Massimo Ferrari und Pierfrancesco Fiorato (Würzburg 2014: 35-84).

${ }^{28}$ Hans Wagner: Philosophie und Reflexion (München, Basel 1980: 233).

${ }^{29}$ ebd. §26, spez. S. $244 \mathrm{ff}$.

${ }^{30}$ Werner Flach: Grundzüge der Ideenlehre. Die Themen der Selbstgestaltung des Menschen und seiner Welt, der Kultur (Würzburg 1997) Kap. 4. Vgl. auch Werner Flach: „Thematik, Methodik und Systematik der Philosophie“. In: Geltung und Begründung. Perspektiven der Philosophie Werner Flachs, hg. von Thomas Göller und Christian Krijnen (Würzburg 2007: 115150, 131-135).

${ }^{31}$ Vgl. H. Wagner: Philosophie und Reflexion, a.a.O. [Anm. 28] §25.; W. Flach: Ideenlehre, a.a.O. [Anm. 30] Kap. 3.

32 Die Freiheitskonzeption der kantianisierenden Transzendentalphilosophie ist allerdings mit grundlegenden internen Schwierigkeiten verbunden, die sich aus der Mehrdimensionalität des Freiheitsbegriffs (etwa der logischen und realen Dimension) ergeben. Vgl. dazu C. Krijnen: „Dasein der Freiheit“, a.a.O. [Anm. 27]; Christian Krijnen: „Kants Kategorien der Freiheit und das Problem der Einheit der Vernunft“. In: Die „Kategorien der Freiheit“ in Kants praktischer Philosophie. Historisch-systematische Beiträge, hg. von Stephan Zimmermann (Berlin 2016: 309-332).
} 
Konform mit der generellen südwestdeutschen Kritik an Kants Architektonik der Vernunft, moniert Bauch, dass auch Kant, wie vorwiegend in der Tradition, das Reich der Freiheit dem der Natur als dem Reich kausaler Notwendigkeit entgegensetzt. ${ }^{33}$ Zugleich jedoch wolle Kant diesen Gegensatz überwinden, wobei Bauch offenbar an Kants Kritik der Urteilskraft denkt (GE, 260): Die verschiedenen Gesetzgebungen der Natur und Freiheit beruhen auf „einem und demselben Boden der Erfahrung“, wobei die eine die andere nicht „stört“: ihr Verhältnis ist das des „Zusammenbestehens“ (KU, V 175). Dieses Zusammenbestehen ist zwar gemäß der Kritik der reinen Vernunft denkbar, es gilt allerdings noch zu begreifen, wie Freiheit in der Sinnenwelt realisiert werden kann, d. h., die Natur muss so gedacht werden können, „daß die Gesetzmäßigkeit ihrer Form wenigstens zur Möglichkeit der in ihr zu bewirkenden Zwecke nach Freiheitsgesetzen zusammenstimme“ (KU, V 176). So mag man Bauch darin folgen, dass Kant in der Kritik der Urteilskraft auf eine "Synthese“ des „ursprünglichen Gegensatzes“ aus ist, letztlich auf eine Synthese der beiden ersten Kritiken. Zugleich ist Bauch aber der Auffassung, Kant arbeite weniger diese Synthese heraus; vielmehr zeige er, dass die beiden Gesetzgebungen eher ungestört „nebeneinander als mit- und ineinander“ bestehen. Bauch zielt dabei vor allem auf die Unterscheidung von Kausalbestimmtheit (,Ursache - Wirkung') und Zweckbestimmtheit (,Zweck - Mittel') im menschlichen Handeln ab (GE, 262 ff.). Im menschlichen Handeln vereinigen sich Kausalbestimmtheit als Notwendigkeit und Zweckbestimmtheit als Freiheit.

Gegen Kant betont Bauch dabei, dass Zwecke nicht als Ursachen konzipiert werden sollten, sondern es sich genauer besehen beim Zweck-Mittel-Verhältnis um die ,Wertabhängigkeit‘ eines Wirklichen handelt. Kausal- und Wertabhängigkeit sind einerseits zu unterscheiden, anderseits jedoch ist ein Wirken nach (oder gegen) Werten immer auch kausalbedingt. Es sind aber nicht die Werte, die als Zweckursachen wirken, sondern ein Wirkliches, das Kausalgesetzen gemäß wirkt und darin zugleich wertbestimmt ist (GE, 263). Ebensolches doppelbestimmtes Wirken eines Wirklichen ist das Wirken der ,Person': die Person kann sich in ihrem immer kausalbestimmten Handeln auf Werte, d. h. Orientierungsdeterminanten vernunftbestimmter Tätigkeit, beziehen. ${ }^{34}$ Die Darstellung oder Realisierung von Werten im Wirklichen ist Hervorbringung von Wirkungen, sodass Wertverwirklichung und Kausalbeziehung, das teleologische und das kausale Verhältnis, zwar verschieden, zugleich jedoch im wertbestimmten Wirken „unabtrennbar“ sind und nur durch eine, wie es heißt, „verfehlte Abstraktion“ getrennt werden können (GE, 263). Für Bauch war Kant in gewissem Umfang einer solchen Abstraktion erlegen, mit der Folge, dass die Frage nach der Vereinigung von Kausalität aus Freiheit und Kausalität der Natur nicht zu einer „befriedigenden Auflösung“ gelangte (GE, 265 f.). Die konstitutive Bedeutung der Kategorien ist bei Kant primär Naturkonstitution, nicht Konstitution des Ganzen der Wirklichkeit. ${ }^{35}$

Bauchs Lösung für das unzureichende Einheitskonzept unterstreicht dann zunächst Kants eigene Forderung der Kritik der reinen Vernunft, die Welt müsse ,als aus einer Idee entsprungen vorgestellt werden“. ${ }^{36}$ Die Welt oder Wirklichkeit ist daher immer schon ideebestimmt und in diesem Sinne nicht sinn- oder wertfrei. Sie kann vielmehr sinnvoll (oder sinnwidrig) werden. Dieses WerdenKönnen ist „Zuletzt“ durch eine besondere „Spontaneität“ innerhalb des Wirklichen charakterisiert, in der der Sinn „unmittelbar“ lebendig wird: es ist die Spontaneität der „Psyche“ und damit des konkreten Subjekts oder der Person (GE, 268). Diese Spontaneität objektiviert sich „mittelbar durch

${ }^{33}$ Vgl. Bruno Bauch: Grundzüge der Ethik [= GE] (Stuttgart 1935: Kap. VII, bes. 259-281)

${ }^{34}$ GE 264 f. Vgl. zu Bauchs Konzept der Person als Wert- oder Geltungsverwirklichungsinstanz eingehend Christian Krijnen: The Very Idea of Organization. Social Ontology Today: Kantian and Hegelian Reconsiderations (Leiden, Boston 2015) Kap. 4; C. Krijnen: „Dasein der Freiheit“, a.a.O. [Anm. 27]; Christian Krijnen: „Der „Kulturimperativ“ als Geltungsverwirklichungsforderung. Hegels Formalismusproblem im Gewand kantianisierender Kulturphilosophie“. In: Kultur. Interdisziplinäre Zugänge, hg. von Hubertus Busche, Thomas Heinze u. a. (Wiesbaden, im Erscheinen).

${ }^{35}$ Vgl. ausführlich Bruno Bauch: Wahrheit, Wert und Wirklichkeit (Leipzig 1923) Teil 3.II mit 4.I, sowie Bruno Bauch: Die Idee (Leipzig 1926). Vgl. dazu C. Krijnen: Philosophie als System, a.a.O. [Anm. 27] Kap. 5.3.

${ }^{36} \mathrm{KrV}$, B843 f., vgl. GE, 268, 271 f., 280. 
die erkennende, handelnde, ästhetische usw. Tätigkeit des Subjekts“ in ein entsprechendes Werk: Wirken wie Werk haben Sinn durch das Subjekt, das sich „spontan“ auf Werte bezieht, sich auf und nach Werten richten und Werten gemäß unmittelbar wirken kann (GE, 269). Darin liegt auch, dass das Wirken des Subjekts nicht nur, wie alles Wirken, kausal bestimmt ist - als Wirken des Subjekts ist es Handeln und Schaffen. Anders gesagt: „Natur“ und „Kultur“ sind beide wirklich und insofern, als Wirklichkeit, ideebedingtheit, also nicht sinnfrei; die Natur ist jedoch frei von „Wertzielen und Aufgaben“, die Kultur nur Kultur als Beziehung des Wirklichen auf Werte. Qua Wirken steht das Kulturwirken zwar auch unter dem Kausalgesetz, anders jedoch als bloßes Naturwirken kann es sich zugleich ,unmittelbar' unter Aufgaben, Werte stellen. Als Wirken nach Werten vereinigt es in sich unmittelbar Kausalität und Freiheit. Solches Wirken muss frei sein, wodurch sich für Bauch „Spontaneität“ als „Freiheit“ auszeichnet; diese Freiheit ist kein „Freisein von der Kausalität“, sondern ein „Freisein zu Aufgaben, Zielen, Werten“ (GE, 270 f.). Durch diese Spontaneität ist das Subjekt Person und Täter einer Tat. Es ist eine Spontaneität, die „Kausalität der Freiheit“ ist (GE, 271). Bauch unterscheidet also sachlich gesehen innerhalb der einen Kausalität, die die Wirklichkeit bestimmt, zwei Arten von Kausalität, will sie jedoch versus Kant nicht als „prinzipiell abgetrennt“ verstanden wissen, sondern betont deren Zusammenhang.

Offenbar rückt Bauch damit die praktische oder personale Freiheit wieder in den Vordergrund. Während er zunächst darauf abzielte, dass Freiheit nicht einfachhin der Natur als dem Reich kausaler Notwendigkeit entgegengesetzt werden dürfe, sondern die Welt vielmehr „als aus einer Idee entsprungen“ vorgestellt werden müsse, läuft Bauchs Lösung bei Lichte besehen nicht sosehr auf diese Idee als Einheit hinaus, sondern auf das konkrete Subjekt oder die Person als Einheit von Natur und Freiheit. Sie läuft damit auf diejenige Einheit von Kausalität und Freiheit hinaus, die eine Handlung oder Tat ist, auf die ,Faktizität‘ der Vernunft also, mag Bauch auch innerhalb einer umfassenden Kausalität zwei spezifische Formen unterscheiden. Der Grund, die umfassende Kausalität, ist nicht selbst als Freiheit gedacht. Die Synthese, die Bauch anvisiert, ist die von Kausalität und Freiheit, nicht die von Natur und Freiheit. ${ }^{37}$ Es ist eine Synthese im „spontanen personalen Wirken“. Kurzum: Bauch denkt Freiheit ,in ' der Natur, nicht die Natur ,als' gegründet in Freiheit, Freiheit nicht als den kosmologischen Grund von Wirklichkeit.

Gewiß, wie Bauch sagt, ist die Natur als naturgesetzliches Dasein zwar nicht frei, jedoch ,sinnbedingt‘. Sie kann daher mittelbar ein Medium für unmittelbar wertbezogenes Wirken sein. Bauch kommt es auf die Synthese von Kausalität und Freiheit in diesem unmittelbar wertbezogenen Wirken an: auf das konkrete Subjekt qua sinnlich-geistiges Wesen. Dass unmittelbar wertbezogenes Wirken überhaupt möglich ist und die Natur mittelbar Medium für die Gestaltung der Wirklichkeit nach Maßgabe von Werten sein kann, hat für Bauch, wie gesagt, seinen „letzten und tiefsten Grund“ darin, dass Natur und Kultur, die Totalität der Wirklichkeit qua „Welt aus einer Idee entsprungen“, sinn- oder ideebedingt ist. Freiheit ist von Bauch nicht als kosmologische Grundlage der Welt konzipiert; sie ist für ihn vielmehr „kausale Bestimmtheit nach objektiven Zwecken als sich selber bestimmendes Wirken nach objektiven Zwecken, weil nach Aufgaben, Zielen und Werten“: nicht Zwecke wirken, sondern das „spontane Subjekt“ als Person wirkt nach Zwecken bzw. Aufgaben, Zielen und Werten (GE, 272). ${ }^{38}$ Die Synthese, auf die es Bauch zufolge „ursprünglich“ (GE, 275) oder „unmittelbar“ (GE, 272) ankommt, ist die von Kausalität und Freiheit im konkreten Subjekt, nicht die von Natur und Freiheit.

Zugleich jedoch denkt sich Bauch die Welt ,als aus einer Idee entsprungen“, als eine Welt, die sich in „Stufen“ entfaltet (von der anorganischen zur organischen Natur und schließlich zur

${ }^{37} \mathrm{GE}, 271 \mathrm{f} ., 275,278$

${ }^{38}$ Das konkrete Subjekt ist im südwestdeutschen Neukantianismus in logischer Hinsicht die Vermittlungsfunktion zwischen Wert und Wirklichkeit, gleich ob bei Rickert, Lask oder Bauch etwa. Vgl. dazu C. Krijnen: Nachmetaphysischer Sinn, a.a.O. [Anm. 27] Kap. 6.2.1 u. 6.2.3.3 
Kultur) (GE, 280 f.). Diese „Selbstentfaltung“ der Idee soll die Konzeption von zwei „getrennten“ Reichen der Natur und Freiheit zugunsten eines „,mit- und ineinander“ Bestehens überwinden, sodass beide Gesetzgebungen der Vernunft bzw. Reiche der Natur und Freiheit einer grundlegenden „übergreifenden Einheit“ entstammen. Als übergreifende Einheit bringt Bauch entsprechend die Idee ins Spiel. ${ }^{39}$ Sie ist Grund und Ziel des Wirklichkeitsganzen; aus ihr entspringt in einem nichtzeitlichen Sinne die Wirklichkeit, wodurch ein zeitliches Entstehen allererst möglich wird, wie, sozusagen, aus Kants kosmologischer Freiheit die Erscheinungswelt entsteht. Freiheit indes ist für Bauch nicht dieser Ursprung. Sie ist ihm zwar keine „psychologische Eigenschaft“" oder ein seelisches „Naturvermögen“, sondern ein „Wertprädikat“ bzw., wie er mit Bezug auf Kant formuliert, ein „transzendentales Prädikat“ (GE, 283). Dennoch ist dieses transzendentale Prädikat keines jener Ursprungseinheit, die die Idee ist. Das konkrete Subjekt bildet die Synthese von Kausalität und Freiheit. Wie Bauch auch sagt: die Kultur, nicht die Natur ist der „logische Ort“ der Freiheit, und er fügt hinzu: wir begegneten diesen Gedanken bei Kant wie bei Hegel (GE, 285). Bauch unterschlägt dabei allerdings, dass die Sache bei Kant und Hegel anders gelagert ist: Freiheit als transzendentales Prädikat, d. h. transzendentale Freiheit, ist für Kant zunächst kosmologische Freiheit und als solche eine Freiheit, die der Natur und der Kultur noch zugrunde liegt. Sie hat also bei Kant nicht ihren ,logischen' Ort in der Kultur. Ebensowenig ist bei Hegel der logische Ort der Freiheit die Sphäre des Geistes: der logische Ort der Freiheit ist das Logische, d. h. die Idee im Element des reinen Denkens. Bauchs Synthese von Natur und Freiheit hingegen ist die des handelnden und darin Kultur schaffenden Subjekts. Bauch denkt Freiheit somit als praktische Freiheit, als personales Wirken, nicht als transzendentale Freiheit im kosmologischen Sinne. Menschliches Handeln vereint Natur- und Freiheitskausalität.

Diese Verwischung des Einheitsgedankens als Idee, aus der die Welt entspringt, und als Subjekt, in der sich Kausalität und Freiheit und damit, als Kultur, Natur und Freiheit vereinen, ist dem axiotischen Grundverhältnis verdankt. Hier ist die Darstellung der Idee als Tätigkeit eines konkreten Subjekts konzipiert, das sich gemäß ihm intrinsisch zugehörigen Werten gestaltet, sich selbst also gestaltet und in eins damit die Wirklichkeit als Kultur. Freiheit ist Freiheit des Subjekts; sie macht seine Möglichkeit und Aufgabe aus. Damit reproduziert sich das Kantische Modell der Freiheit als Kausalität. Sie ist nicht Manifestation eines in sich differenzierten Einen, das in allem anderen bei sich selbst ist und bleibt, sondern setzt es voraus.

\section{Literatur}

BAUCH, Bruno: Die Idee, Leipzig, 1926

: Grundzüge der Ethik, Stuttgart, 1935.

: Wahrheit, Wert und Wirklichkeit, Leipzig, 1923

BAUMGARTEN, Alexander Gottlieb: „Metaphysica“. Ed. IV, 1757. In: Kants gesammelte Schriften. Bd. I-XXIX XIV, XV, XVII, hg. von Königlich-Preußische Akademie der Wissenschaften et al., Berlin, $1900 \mathrm{ff}$.

BIANCO, Bruno: „Freiheit gegen Fatalismus. Zu Joachim Langes Kritik an Wolff“. In: Zentren der Aufklärung: I: Halle. Aufklärung und Pietismus, hg. von Norbert Hinske, Berlin, 1989, 111-155.

BEUTEL, Albrecht: „Causa Wolffiana. Die Vertreibung Christian Wolffs aus Preußen 1723 als Kulminationspunkt des theologisch-politischen Konflikts zwischen halleschem Pietismus und Aufklärungsphilosophie“. In: Reflektierte Religion. Beiträge zur Geschichte des Protestantismus, Tübingen, 2007, 125-169.

\footnotetext{
${ }^{39}$ Vgl. noch GE, 271, 272, 278, 280 f.
} 
FINSTER, Reinhard: „Spontaneität, Freiheit und unbedingte Kausalität bei Leibniz, Crusius und Kant“, Studia Leibnitiana 14 (1982), 266-277.

FINSTER, Reinhard: Spontaneität und Freiheit. Eine Untersuchung zu Kants theoretischer Philosophie unter Berücksichtigung von Leibniz, Wolff und Crusius. Univ. Diss., Trier, 1984.

FLACH, Werner: Die vielfältigen Aspekte des Freiheitsbegriffs in der Philosophie Kants (unveröff. Manuskript), 2016.

: Grundzüge der Ideenlehre. Die Themen der Selbstgestaltung des Menschen und seiner Welt, der Kultur, Würzburg, 1997.

: Kant zu Geschichte, Kultur und Recht. Hrsg. von Wolfgang Bock, Berlin, 2015.

: „Thematik, Methodik und Systematik der Philosophie“. In: Geltung und Begründung. Perspektiven der Philosophie Werner Flachs, hg. von Thomas Göller und Christian Krijnen, Würzburg, 2007, 115-150.

FULDA, Hans Friedrich: „Der eine Begriff als das Freie und die Manifestationen der Freiheit des Geistes“. In: Hegel - 200 Jahre Wissenschaft der Logik, hg. von Anton Friedrich Koch, Friedrike Schick u. a., Hamburg, 2014, 15-41.

: „Freiheit als Vermögen der Kausalität und als Weise, bei sich selbst zu sein“. In: Inmitten der Zeit. Beiträge zur europäischen Gegenwartsphilosophie (FS M. Riedel), hg. von Thomas Grethlein und Heinrich Leitner, Würzburg, 1996, 47-63.

GEISMANN, Georg: „Kant über Freiheit in spekulativer und in praktischer Hinsicht“. In: Kant und kein Ende. Band 1: Studien zur Moral-, Religions- und Geschichtsphilosophie, Würzburg, 2009, 119-142.

HEIMSOETH, Heinz: „Zum kosmotheologischen Ursprung der Kantischen Freiheitsantinomie“, Kant-Studien 56 (1966), 206-229.

HEGEL, Georg Wilhelm Friedrich: Enzyklopädie der philosophischen Wissenschaften im Grundrisse (1830). Hg. von Friedhelm Nicolin und Otto Pöggeler, Hamburg, 1991.

: Grundlinien der Philosophie des Rechts.Hg. von Johannes Hoffmeister, Hamburg, 1955.

: Phänomenologie des Geistes. Hrsg. von Wolfgang Bonsiepen und Reinhard Heede, Hamburg, 1980.

: Wissenschaft der Logik. Hg. von Georg Lasson, Leipzig, 1951.

HÖFFE, Otfried: Kants Kritik der reinen Vernunft. Die Grundlegung der modernen Philosophie, München, 2003

KAWAMURA, Katsutoshi: „Eine begriffsgeschichtliche Skizze der "Willkür" im 18. Jahrhundert. Wolff, Wagner, Feder und Kant". In: Recht und Frieden in der Philosophie Kants. Akten des X. Internationalen Kant-Kongresses III, hg. von Valerio Rohden, Ricardo Terra u. a., Berlin, 2008, 173-181.

: Spontaneität und Willkür. Der Freiheitsbegriff in Kants Antinomienlehre und seine historischen Wurzeln, Stuttgart-Bad Cannstatt, 1996.

KNAPPIK, Franz; Im Reich der Freiheit. Hegels Theorie autonomer Vernunft, Berlin, 2013.

KRIJNEN, Christian: „Das Dasein der Freiheit. Geltungsrealisierung bei Hegel und in der kantianisierenden Transzendentalphilosophie“. In: Kulturphilosophie. Probleme und Perspektiven des Neukantianismus, hg. von Christian Krijnen, Massimo Ferrari und Pierfrancesco Fiorato, Würzburg, 2014, 35-84. 
: „Der „Kulturimperativ“ als Geltungsverwirklichungsforderung. Hegels Formalismusproblem im Gewand kantianisierender Kulturphilosophie“. In: Kultur. Interdisziplinäre Zugänge, hg. von Hubertus Busche, Thomas Heinze u. a., Wiesbaden, im Erscheinen.

: „Freiheit als ursprüngliche Einheit der Vernunft. Hegels begriffslogische Lösung eines Kantischen Problems“. In: Natur und Geist, hg. von Wolfgang Neuser und Pirmin StekelerWeithofer, Würzburg, 2016, 25-52.

: „Kants Kategorien der Freiheit und das Problem der Einheit der Vernunft“. In: Die „Kategorien der Freiheit“ in Kants praktischer Philosophie. Historisch-systematische Beiträge, hg. von Stephan Zimmermann, Berlin, 2016, 309-332.

: Nachmetaphysischer Sinn. Eine problemgeschichtliche und systematische Studie zu den Prinzipien der Wertphilosophie Heinrich Rickerts, Würzburg, 2001.

: Philosophie als System. Prinzipientheoretische Untersuchungen zum Systemgedanken bei Hegel, im Neukantianismus und in der Gegenwartsphilosophie, Würzburg, 2008.

: „Sittlichkeit and the Actuality of Freedom“, in: The Palgrave Hegel Handbook, ed. M. Bykova and K. Westphal, Houndmills, Basingstoke, Hampshire (im Erscheinen).

: The Very Idea of Organization. Social Ontology Today: Kantian and Hegelian Reconsiderations, Leiden-Boston, 2015

LEIBNIZ, Gottfried Wilhelm: Essais de théodicée sur la bonté de dieu, la liberté de l'homme et l'origine du mal. In: Die philosophischen Schriften von Gottfried Wilhelm Leibniz VI, hg. von Carl Immanuel Gerhardt, Hildesheim, 1978.

SCHWAIGER, Clemens: Alexander Gottlieb Baumgarten. Ein intellektuelles Porträt. Studien zur Metaphysik und Ethik von Kants Leitautor, Stuttgart-Bad Cannstatt, 2011.

WAGNER, Hans: „Die kosmologische Antithetik und ihre Auflösung in Kants Kr.d.r.V“. In: $Z u$ Kants Kritischer Philosophie, hg. von Bernward Grünewald und Hariolf Oberer, Würzburg, 2008, 82-97.

: „Kants ergänzende Überlegungen zur Möglichkeit von Freiheit im Rahmen der Auflösung der dritten Antinomie“. In: Zu Kants Kritischer Philosophie, hg. von Bernward Grünewald und Hariolf Oberer, Würzburg, 2008, 98-106.

: Philosophie und Reflexion, München, Basel, 1980.

WOLFF, Christian:Vernünffige Gedancken von Gott, der Welt und der Seele des Menschen, auch allen Dingen überhaupt. In: Gesammelte WerkeI. 2, hg. von École, Jean et al., Hildesheim, New York, 1962 ff. 


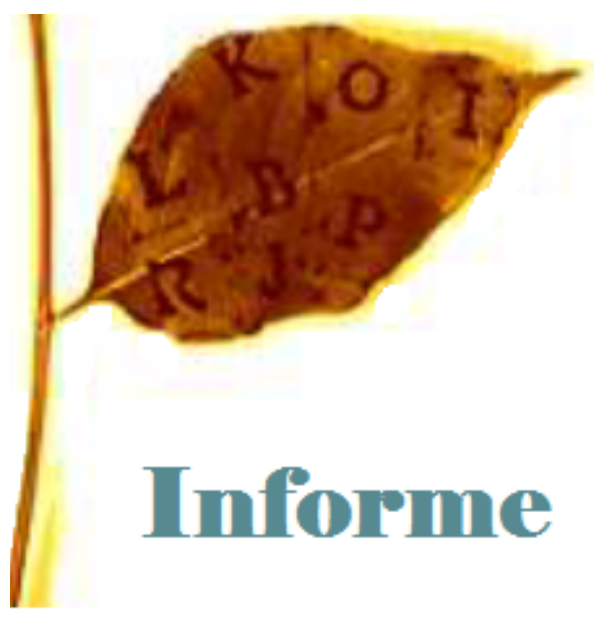




\title{
VII Coloquio-Multilateral-Kant 2017
}

\author{
PAULA ÓRDENES AZÚA ${ }^{1}$
}

Entre el 28 y 30 de abril del presente año tuvo lugar en la ciudad alemana de Halle (Saale) la séptima versión del Multilateral-Kant-Coloquio. En esta oportunidad, el evento tuvo como temática de su convocatoria a 'Kant y sus críticos', y contó con la presencia de más de cincuenta especialistas dedicados al estudio de la filosofía kantiana provenientes de América, Europa y Asia.

En la Martin-Luther-Universität Halle-Wittenberg y durante tres días se celebró el encuentro multilateral en torno a la recepción crítica de la obra de Kant. El lugar de encuentro fue idóneo para esta actividad, puesto que por este histórico lugar pasaron pensadores que contribuyeron tanto a la formación - Christian Wolff, Alexander Baumgarten, Franz A. Schultz, entre otros- como a la recepción inmediata del pensamiento de Kant -A. Eberbach, L. M. Träger, L. H. von Jakob, J. H. Tiefrunk, J. S. Beck-. Como si lo anterior no fuera motivo suficiente, Halle es una ciudad que cuenta con una larga tradición dedicada a los estudios de la filosofía kantiana, puesto que en ella fueron fundados en 1896 los 'Kant-Studien', mientras que en 1904 - con ocasión del centenario del fallecimiento de Kant - nació la 'Kant-Gesellschaft' y finalmente, durante el 2014, fue creado el 'Immanuel-Kant-Forum'.

\section{8 de abril de 2017}

La conferencia inaugural fue realizada por el profesor Walther Ch. Zimmerli, de la HumboldtUniversität de Berlin, y llevó por título: "Kant 'behind our backs': Time and (time-)consciousness today". Zimmerli expuso sobre las divergencias teóricas que se desglosan según la concepción del tiempo a considerar. En este caso, y como punto de deliberación, abordó la filosofía kantiana (tras el giro copernicano) versus las ciencias físico-biológicas, enfatizando las diferencias que se desprenden desde una teoría de la conciencia pura frente a una teoría de la evolución de la conciencia. Esta última tendría por fundamento, no obstante, uno empírico y no uno a priori, ya que la conciencia sería producto de un proceso evolutivo que generaría seres vivos capaces de reflexionar en algún momento sobre sí y sobre el tiempo (a saber, conciencia del nowness).

Concluida la charla magistral, se dio inicio a las secciones de conferencias paralelas. Las primeras tres presentaciones - simultáneas - fueron: “A serious blow to the Kantian Critique': Mendelssohn and the B Edition", realizada por Corey Dick (Univ. Of Western Ontario/MLU HalleWittemberg); "Kant and Constant on Lying", presentada por Joel Thiago Klein (Univ. Rio Grande do Norte) y "Kants These zum Sein als Position und ihre Kritik durch Hegel", por Héctor Ferreiro (Pontificia Univ. Católica de Buenos Aires).

Tras una pausa prosiguió el congreso con dos secciones paralelas más. El auditorio A del histórico edificio Melanchthonianum albergó dos presentaciones sobre la discusión suscitada a partir de la crítica escéptica de Schulze a la filosofía teórica de Kant. Reinhard Brandt (Philipps-UnivMarburg) presentó una ponencia titulada: 'Lassen sich die 'Anmaßungen der Vernunftkritik' gegen den Skeptizismus (Aenesidemus-Schulze) durch die Rechtsgrundlage der Transzendentalen Deduktion ("quid juris?") verteidigen?" y Falk Wunderlich (MLU Halle-Wittenberg) expuso sobre "Kant and Schulze: The challenge of direct realism".

En el auditorio B la discusión se centró, a su vez, en torno a la reflexión sobre las críticas a la filosofía práctica. El primero en presentar fue Bas Tönissen (Univ. St. Andrews), con la ponencia

\footnotetext{
${ }^{1}$ Universität Heidelberg.
} 
"The paradox of method in the Critique of Practical Reason: Kant's reply to H.A. Pistorius". Luego fue el turno de Valentina Dafne de Vita (MLU Halle-Wittenberg), quien presentó el texto "Die zeitgenössischen Kritiker an Kants Pflichten gegen sich selbst”.

En el auditorio $\mathrm{Z}$ las presentaciones estuvieron dedicadas a la discusión sobre la filosofía del derecho de Kant. Fiorella Tomassini (Univ. de Buenos Aires) expuso la ponencia "Die Einteilung der Rechtspflichten nach den Ulpian-Formeln", mientras que Gabriel Rivero (Univ. Mannheim) presentó el texto "Kants Eigentumsrecht und seine Kritiker".

Tras la pausa de almuerzo continuó una ronda de sesiones paralelas, dedicada esta vez al vínculo teórico, ético y político entre Kant y otros filósofos. El auditorio A contó con la presencia del profesor Manfred Baum (Bergische Univ. Wuppertal), autor de la ponencia "Über Kant und Eberhard". En el auditorio B, Paulo Renatus Jesus (Univ. Lisboa) presentó, a su vez, el texto "Kant et Lévinas sur l'origine de la loi morale" y en el auditorio Z, Gualtiero Lorini (Technische Universität Berlin) expuso sobre "Recht, Sittlichkeit und Religion: eine politische Debatte? Einige Vergleichselemente zwischen Kant, Fichte und Hegel".

Antes de finalizar la jornada del primer día hubo una sesión doble en el auditorio A, dedicada a las críticas de Maimon al kantismo. Por un lado, el profesor Mirosław Żelanzy (Univ. Toruń) presentó el texto "Das Verhältnis von Salomon Maimon zu Kant im Lichte der Korrespondenz und der neu entdeckten Manuskripte", y por otro, Hernán Pringe (Univ. Buenos Aires/Universidad Diego Portales, Santiago de Chile) leyó su ponencia "Criticism of Kant's Doctrine of Mathematical Cognition and the Possibility of Metaphysics as a Science". Las dos presentaciones del auditorio B, por su parte, se dedicaron a contrastar la ética kantiana con las de Schiller y Schopenhauer: Charles Feldhaus (Univ. Londrina) presentó: "Kant, Schiller, Obligation and Chimerical Ethics" y Margit Ruffing (Johannes Gutenberg Univ. Mainz): “'Mysterium der Ethik' vs 'corpus mysticum' Moralitätsverständnis bei Schopenhauer und Kant". Las exposiciones del auditorio Z fueron: "Kant und die Gefahr des Determinismus", de Emanuele Cafagna (Univ. Chieti-Pescara) y "Kant über die moralische Qualität physischer Selbsterhaltung als Kritik an Stoa und die Gegenkritik durch Jakob Friedrich Fries", leída por Katerina Mihaylova (MLU Halle-Wittenberg).

Finalmente, se realizó una recepción del congreso en la alcaldía de la ciudad de Halle. En ella se comunicó que el próximo Multilateral será durante el segundo semestre del 2018 en la ciudad de Catania y el subsiguiente en la ciudad de Kaliningrado (nombre actual para la ciudad natal de Kant: Königsberg).

29 de abril

E1 sábado 29 de abril continuó el encuentro bajo la modalidad de tres sesiones dobles y paralelas por la mañana. En el auditorio A se presentó, en primer lugar, el profesor invitado Ubirajara Rancan de Azevedo Marques (Universidade Estadual Paulista, Campus de Marília), con la ponencia titulada "Uma anotação de Kant acerca dum exemplo musical de Eberhard sobre o "fundamento das representações"” y, en segundo lugar, Fernando M. F. Silva (Univ. Lisboa), con el texto "Novalis, Critic of Kant". En el auditorio B se centró la discusión en la crítica de Reinhold a la filosofía práctica de Kant. Presentaron sus textos, en primer lugar, Jörg Noller (LMU München) con "'Practical Reason is not the Will': Kant and Reinhold's Dilemma" y, en segundo, Antonino Falduto (MLU HalleWittenberg), con la ponencia "Reinhold and Kant on Freedom and the Evil". En el auditorio Z las discusiones se centraron en torno a la estética kantiana: Mònica Carbó Ribugent (Univ. Girona) expuso "On Beauty, Truth and Philosophy: Kantian aesthetical legacy" y Anna-Maria C. Bartsch (LMU Univ. München) presentó el texto "Kants ästhetische Urteilstheorie und die Kritik der Formalen Ästhetik nach Zimmermann.”

Después de una pausa continuaron tres sesiones paralelas dobles más. Una presentación al mediodía patrocinada por la "Marie Sklodowska-Curie" Fellowship fue realizada por Luigi Caranti (Univ. Catania) en el auditorio D. En el auditorio A, al mismo tiempo, expusieron Zdravko Kobe (Univ. Ljubljana) con "Kant und seine Kritiker. Der Fall Jacob Sigismund Beck" y Fernando Moledo (Univ. Buenos Aires y Univ. MLU Halle-Wittenberg), quien presentó la ponencia "Kants Auffassung des Menschen als Zweck an sich selbst und der Formalismus-Vorwurf gegen die kritische 
Philosophie". En el auditorio B expusieron, en primer lugar, el profesor invitado Jens Timmermann (Univ. St. Andrews), con el texto "“Nichts ist schädlicher al seine Ethik laxa'- Kant und der Vorwurf der moralischen Überförderung" y, en segundo lugar, Maria de Lourdes Borges (Univ. Santa Catarina), quien leyó "The feminist criticism of Kant's philosophy". En el auditorio Z se presentó Ansgar Lyssy (LMU München) con una ponencia sobre "Der Despot als philosophisches Problem Friedrich Bouterweks Kritik an Kants politischer Philosophie" y Esther Oluffa Pedersen (Roskilde Univ.) lo hizo con la ponencia "The Deluged heart of the Idle Cosmopolite is a Dwelling for Nobody. How Herder urged Kant to think through teleology".

Con estas presentaciones se dieron por terminadas las sesiones paralelas del segundo día y se abrió paso al programa cultural que, en primer lugar, comprendía conocer la 'Franckesche Stiftung' $\mathrm{y}$, posteriormente, una visita a la casa de Christian Wolff, donde también se expuso sobre la relevancia histórica de la ciudad de Halle como privilegiado centro de desarrollo académico y filosófico durante la Ilustración.

Heiner F. Klemme, Corey Dyck y Falk Wunderlich con su exposición titulada "Philosophy in Halle (1696 -): A Hotspot of Enlightenment", dieron cuenta de un listado de personajes eminentes y relevantes que transitaron por la ciudad y Universidad de Halle desde 1696 hasta la actualidad. Entre ellos destaca la primera mujer en obtener un doctorado en Alemania: Dorothea Christiane Erxleben (Leporin). A pesar de los múltiples obstáculos que tuvo que vivenciar para llevar a cabo su carrera como médico y obtener su doctorado, resulta ser bastante significativo que una mujer en esa época haya sido capaz de -0 , mejor dicho, que se le haya permitido- ${ }^{2}$ desarrollarse académicamente en la Universidad de Halle. Un signo que invita a pensar la ciudad de Halle como centro cultural; un lugar poseedor de una mentalidad comparativamente más avanzada - puesto que muestra un progreso, no sólo intelectual, sino también moral—en relación al resto de la región.

30 de abril

El último día de la actividad internacional organizada por Heiner Klemme y Antonino Falduto contó con nueve sesiones paralelas y concluyó con la presentación de un libro.

La primera ronda de ponencias en el auditorio A comenzó con la participación de Kurt Röttgers (Fernuniversität Hagen), quien comunicó la ponencia "Frühe Kant-Kritik von der Sprachlichkeit her", mientras que el profesor invitado Thomas Sören Hoffmann (Fernuniversität Hagen) presentó el texto "Kritik und Metakritik: Zu Hamanns Kantlektüren". En el auditorio B expuso el profesor invitado Luigi Caranti (Univ. Catania) sobre "The Kantian Federation and its critics". En el auditorio Z, al mismo tiempo, se desplegó una discusión a partir del pensamiento de dos representantes del idealismo alemán (Fichte y Hegel) sobre la filosofía kantiana: Manja Kisner (LMU München) leyó su ponencia "Fichtes metaphysische Rezeption des kantischen transzendentalen Idealismus" mientras que el profesor invitado Dietmar Heidemann (Univ. Luxemburg) concluyó la mesa con su texto "Hegels Kritik an Kants Ding an sich in der 'Wissenschaft der Logik"”.

Luego de la primera pausa del día continuaron las sesiones paralelas dobles. En el auditorio A expusieron, en primer lugar, Ileana Beade (Univ. Buenos Aires) con el texto "Some observations regarding Hamann's critique of Kant's distinction between the public and the private use of reason" y, en segundo lugar, el profesor invitado Saneyuki Yamatsuta (Univ. Tokyo), quien presentó la ponencia "Subjektivität oder Intersubjektivität? Tetsuro Watsujis Kritik der kantischen Ethik". En el auditorio B, a su vez, presentaron, Nuria Sánchez Madrid (Univer. Complutense Madrid) quien expuso sobre "Kantian vs. Current Defenses of Human Rights: Some Critical Remark" y luego, el profesor invitado

${ }^{2}$ Ha de ser mencionado que quizás sin el apoyo del rey de Prusia, Friedrich II (Federico el Grande), quien ordenó a la Universidad que aceptara a Leporin en la inscripción de su doctorado, no habría sido posible su acceso universitario, pues la formación médica recibida por Dorothe se realizó de forma privada (de otra manera no habría sido factible en la época). Tras la ayuda del rey y antes de la obtención de su doctorado en 1754 (Ouod nimis cito ac iucunde curare saepius fiat causa minus tutae curationis), publicó en 1742 el artículo "Gründliche Untersuchung der Ursachen, die das weibliche Geschlecht vom Studiren abhalten". En el texto mencionado investiga las razones (o prejuicios) que justificaron mantener a las mujeres fuera del ámbito universitario. En caso de interés, en el siguiente enlace se puede encontrar la obra escaneada de Dorothe Leporin:

https://commons.wikimedia.org/wiki/Gr\%C3\%BCndliche Untersuchung der Ursachen, die das weibliche Geschlecht vom Stu diren abhalten 
Anselmo Aportone (Univ. Roma Tor Vergata), con la comunicación "Synthesis bei Kant und Strawson". Tras la ponencia de Dahan Fan (Eberhard-Karls-Univ. Tübingen) en el auditorio Z y que llevó por título "Zwei gegenläufige Modelle in Kants Ästhetik? Eine Verteidigung im Hinblick auf Schönheit", expuso el profesor invitado Luca Fonnesu (Univ. Pavia) sobre "Die Kritik der Kantischen Prinzipienethik bei Fichte und Hegel".

Posterior a la pausa de almuerzo hubo cinco rondas de sesiones paralelas. En el auditorio A expuso el profesor invitado Fuling Wang (Renmin Univ., Beijing), sobre "Kant's philosophy in the perspective of Confucianism". Presentaron en el auditorio B, en primer lugar, Tomasz Kupś (Univ. Toruń) su ponencia "Institutioneller Antikantianismus an der Kaiserlichen Universität Vilnius. Wettbewerb an dem Philosophie-Lehrstuhl in den Jahren 1821-1822" y, en segundo lugar, Jitka Paitlová (Univ. Pilsen) con el texto "Karl Poppers Kritik an Kant". Las ponencias del audiorio Z estuvieron a cargo de Martin Sticker (Trinity College Dublin), quien leyó su "Common Human Reason and the Fact of Reason" y del profesor invitado Jacinto Rivera de Rosales (Univ. E. D., Madrid), que expuso su texto sobre "Die Kritiken des neuen Realismus an Kant".

Luego de una breve pausa se efectuó la última ronda de secciones paralelas. El auditorio A contó con las presentaciones de Alexei Krouglov (Univ. Moskau) como profesor invitado y quien leyó su "Kants Kritik von Vladimir Solowjow". Luego sería el turno de Sandra Zákutná (Univ. Prešov), autora de la ponencia "The Reception and Criticism of Kant's Philosophy in the Region of Today's Slovakia at the Turn of the 18th Century and in the First Half of the 19th Century". Finalmente, Marita Rainsborough (Univ. Hamburg / Univ. Kiel) presentó el texto "Kant revisited. Die kritische Auseinandersetzung mit Kants Universalismus und Kosmopolitismus in der afrikanischen Philosophie".

En el auditorio B expusieron Irene Breuer (Bergische Univ. Wuppertal) con "Kant und Husserl: Ding an sich und Telos des Erfahrungsprozesses"; Hsin-Pai Chen (Eberhard Karls-Univ. Tübingen), quien leyó su "Abschied von Kant? - Ulrich Klugs Kritik und die Rolle des Wiedervergeltungsrechts in Kants Rechtsdenken" y, por último, el profesor invitado Gabriele Tomasi (Univ. Padova) que expusó su texto "On Greenberg on Kant and the common sense".

Finalmente, las últimas tres ponencias del auditorio $\mathrm{Z}$ fueron: "From mysticism to metaphysics: Kant and his critics in the last decades of the 19th century" de Tinca Pruneabretonnet (Univ. Bucharest); "Gerold Prauss' Kritik an der Linienanalogie in Kants Zeittheorie", de Bernd Dörflinger (Univ. Trier) y "Plato on Revolution. Political Conservativism in Kant and J. G. Schlosser”, del profesor invitado Günter Zöller (LMU Univ. München/VIU Venice).

Hans-Peter Nowitzki (FSU Jena), Udo Roth (LMU Univ. München), Gideon Stiening (RKU Heidelberg/LMU Univ. München) y Falk Wunderlich finalizaron la séptima versión del ColoquioMultilateral con la presentación del libro: “...wie jeder vernünftige Christ, Socinianer”. Der Briefwechsel des Göttinger Materialisten Michael Hißmann.

Cómputos finales: más de cien asistentes, expositores de más de catorce países, quince profesores invitados, sesenta presentaciones programadas (de las cuales sólo dos no tuvieron lugar), exposiciones en cuatro idiomas diferentes (treinta presentaciones en alemán, veintiséis en inglés, una en francés y una en portugués. Todo indica que el resultado del VII. Multilateral-Kant-Coloquio fue realmente un éxito.

Por último, cabría mencionar que sería interesante poder oír en un futuro encuentro internacional de este tipo no sólo presentaciones en alemán, inglés, francés y portugués sino también en español. Ojalá la lengua castellana vaya tomando fuerza en los estudios filosóficos kantianos y sea considerada una lengua digna de transmisión de ideas, tanto por sus hablantes como por la comunidad académica global. 


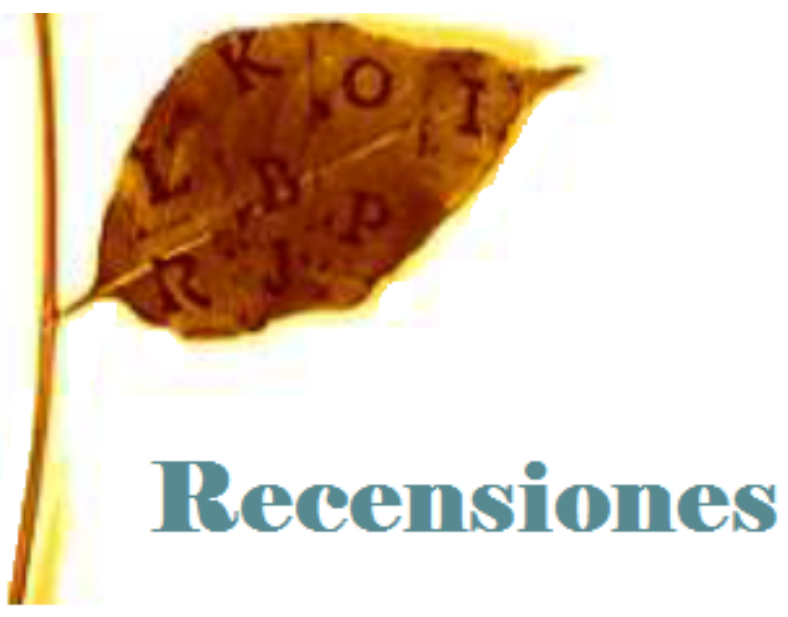




\title{
Alfredo Ferrarin: Il pensare e l'io. Hegel e la critica di Kant. Roma, Carocci Editori, 2016, 244 pp. ISBN: 978-88-430-8246-9.
}

\author{
LUIGI FILIERI $^{1}$
}

In this carefully argued study, Alfredo Ferrarin sets out to renew our understanding of Hegel's idealism. Between pages 24 and 30 of the introduction the author shows how Hegel aimed at subverting what seems to be commonly known, by continuously renewing the meanings of all concepts. In this book, Ferrarin shares the same goal: he aims at proving that many recent common interpretations of Hegel are based on arbitrary and implicit presuppositions and readings which are often constructed to contribute to current philosophical debate rather than a proper understanding of Hegel. Though this is not the sole purpose of Ferrarin's study, it clearly represents one of its main methodological aims. Accordingly, all five chapters address Hegel's idea of critique, intended as his philosophical method. The topics covered by the study are: i) the distinction between the idea of subjectivity and the idea that all philosophy must begin with the concept of the "I" (on the presupposition that all thought activity is exercised by the "I"); ii) the discussion of Hegel's account of thinking which cannot be reduced to the activity of the "I" (least of all to the activity of the human being); iii) the spontaneity of thought and its reification in the form of finite determinations; iv) the relationship between "representation" and "concept"in Hegel's Logic. Chapter five deserves a brief specific note. It indeed contains a comparison between Kant's and Hegel's respective notions of philosophy. More precisely, Ferrarin describes Hegel's critique of Kant as biased and arbitrary and provokingly tries to imagine how Kant might have answered Hegel's objections.

The key topics of chapter one, which deals with Hegel's concept of recognition, are selfconsciousness, individuality and intersubjectivity.The core thesis of this chapter is that recognition does not ground self-consciousness. On the contrary, self-consciousness is preliminary to the possibility of recognition. However, this does not mean that self-consciousness is an original given but rather that self-consciousness is always the self-consciousness of a self-comprehending substance. According to this line of argument, the "I" is not the owner of self-consciousness but a manifestation (among others) of the substance insofar as it comprehends itself as the subject of its own development. Ferrarin stresses that Hegel's account of subjectivity goes beyond the subjectivity of the "I". This subjectivity strives to emerge from unconsciousness but when the "I" tries to rely on its own concept as a starting point, its subjectivity proves partial and evasive. To strengthen this reading, Ferrarin compares Hegel's positions in the Encyclopedia with the Phenomenology of Spirit and criticizes Brandom's useof Hegelian recognition in social terms and Pippin's confusion between recognition and the emergence of the social ethical dimension. Both share the view that selfconsciousness is made possible through recognition, while Ferrarin argues the opposite. A very insightful commentary of the master-slave dialectic (in the Phenomenology) leads us i) to recognize the role of work and fear which are closely intertwined in the development of self-consciousness, ii) to understand why and how self-consciousness is what it is not because it is recognized as such (for this reason, page 67 is the most penetrating of the whole chapter).

If chapter one plays a preparatory role, chapter two brings the conclusions of the preceding pages to a new level of discussion concerning the nature of thinking beyond any human determination. The style of this chapter is remarkably different from the first since Ferrarin here reflects on the addressed issues to show how each element turns into another, e.g., how objective thought turns into subjective thought. This chapter contains a broad range of perspectives on its topic: i) objective thought; ii) the logical that enlivens the world; iii) the realization of the concept in the world. Regarding the first point, the author understands the 'objectivity' of objective thought as

${ }^{1}$ Università di Pisa. 
implying that the "I" of the subject must disappear to avoid altering the development of thinking itself, as it is freed from any particularity. Moreover, Ferrarin points out that the same objectivity refers to the internal movement of thought, as the force and instinct of not being moved (or thought) by anything beyond thought itself. The status of thought is not merely trans-individual but also transhuman, like Anaxagoras's concept of nous. The objective intelligibility of the world cannot be reduced to the activity of this or that subject. However, the author firmly warns us against the danger of realism, which would involve hypostatizing the absolute idea as the operational 'subject' of the whole development of thinking. Hegel's answer to such a danger lies in the affinity of all logical particular determinations. 'Objective thought' is a moment of thought which recognizes itself as the subject of its own movement; the transition from objective to subjective thought (which is not the subjectivism of the particular "I") is internal to the activity of thinking itself. This transition displays the pure logic of thought itself and the logical soul that all particular determinations share. In this activity of Begreifen, intended as the activity of the concept the result, this or that determined concept, is not external to its developmental process (the self-determination of thinking). The concept thus results from its own activity; from the activity of the concept itself. Therefore, the concept cannot be identified with a mental representation. The concept owns and determines us, rather than the other way around. However, with outstanding perspicuity Ferrarin clarifies how such dominion is not oppressive but instead the distinctive mark of the exercise of our own freedom.

We reach in this way the perspective of the logical as 'Being', of the Logic as the selfconsciousness of thought. Once the speculative philosopher has released the living logos from its determinate concretions, the internal principle of all reality displays its pure form, namely that of reason as aiming at self-knowing. The logical is its own content or, more precisely, its form (the Logic) as the self-reflection of the content upon itself. This internal reflection is nothing but dialectic: the contradiction as the root of life, of the internal movement of all concepts. A living being, such as Thought, is marked by lacking something and therefore by having needs and desires. To this negative indigence, the positive answer of the needs of reason is what moves and enlivens reason itself. The compresence of these two moments, i.e., the simultaneity of the negative and the positive, is what Hegel calls the living contradiction. The logical thus takes the shape of a soul; the Logic is nothing but the form of self-knowledge. Once we reach the end of the logic (in the Science of Logic) we could therefore be tempted to take the final form of the Absolute Idea (intended as the abstract form of the concept insofar as it is freed from all particular determinations) as the ground of the whole movement. Ferrarin admonishes us against this move: such a hypostasis would amount to realism; a merely formal knowledge of the idea which does not consider its realizations. This lack of awareness moves us to subjective spirit, which is the third and last methodological perspective of the chapter which concerns the activity of thinking as having itself as its own object.

Per se, the Logic of thinking cannot be separated from its own realizations. Although it has to be understood before its determinations, the determinations are nevertheless essential. Ferrarin thus argues that there is no proper truth if truth does not correspond to its becoming real. Truth can be therefore intended as the being-known as true. The beginning and end of the process thus correspond to each other, although their respective meanings are not identical. The former is an abstraction, while the latter is the realized form of thought itself in its internal movement of self-production and selfspecification. It is then clear why we must intend the concept as the true form that produces its own content, as an immanent cause and an embodied reason. Accordingly, the concept cannot be the starting point since it is always a kind of self-mediation. The real account of truth thus lies in the concept's self-realization, which also includes the falsity and particularity of its determinations.

Despite the anti-subjectivism (i.e. the refusal of the "I" as the thinking subject), the chapter moves to a seemingly paradoxical conclusion: the "I" is the authentic concrete universal. By claiming this, Ferrarin is not referring to a metaphysical subject but rather to the freed concept itself: the pure self-consciousness insofar as it understands itself as the subject of its own development and thereby taking the form of "I". This "I" is a form of unity; it is the form of the object as connected with the form of the subject, the transition to its own truth. In other words, the "I" is the way through which the idea adopts the form of self-consciousness. Continuing in this vein, chapter three is dedicated to the reification and the spontaneity of thought.

Here we immediately see how the movement of thought is marked by spontaneity and reification. The infinite, as a subject, thus assumes finite forms. This being-finite 'is' as a moment of 
the infinite, but 'it is not' independently of the infinite. Both are comprehensible only in their reciprocal reference and cannot be separated. The self-alienation of thought in its own determinations precisely mirrors the dialectic between spontaneity and reification. The life of the concept consists of its own solidification through determination: its reification, meaning by this that the concept pulses into the object and not that the concept is thought as the object. For this reason, Ferrarin argues, thought is identical with its own exposition. At this point arises an important question: how is it possible to grasp the spontaneity of thought along with its passivity? The answer lies in Hegel's account of the self-realization of the concept (Sichselbstentäusserung). The fluidification of the finite concept is thus complementary to the solidification: all empirical determinations must be traced back to the self-determining concept. The concept must recognize its determinate and solid reifications as being temporary, being therefore capable to recognize itself through and within such determinations. Ferrarin is careful and precise in emphasizing this point: the being-other of its realizations is recognized by the concept as something not external nor alien. The passive side of the concept is necessary for the concept to take its spontaneity back. However, since thought is unconscious in its realizations, the task of speculative philosophy precisely consists in proving that in all concretions, thought produces its own being-real. Another question is here raised by Ferrarin (pp. 134-135): if the determinations are internal to the concept, how it is possible to say (as Hegel does in the Encyclopedia) that such determinations are also a product of the thinking subject? Before addressing Hegel's position, Ferrarin firmly criticizes both metaphysical realism according to which such productions are merely illusory and transcendentalism which claims that the 'Idea' itself is one of our productions. On the contrary, Hegel affirms that, although the 'Idea' is irreducible to its finite forms, it can (re-)cognize itself only through finite spirit. The work of thought is that of transforming the given into the concept (more precisely, into 'its own' concept), thereby allowing the concept to recognize itself in the finite. Hegel redefines the meaning of self-consciousness according to this movement. Self-consciousness is no immediate reference to a first-person point of view but rather a completely developed self-knowledge. It is knowledge in terms of relation between truth and certainty; subject and content. Such an account of knowledge thus involves a basic difference between the terms at issue, and for this reason this knowledge can be described only as a development and never as an identity. It is rather a form of unity, intended as the immanence of thought to its own determinations.

Chapter four begins by stating that the reversal of the ordinary subjective view of thought requires us to investigate the notion of representation. This notion has the same content as the concept, but a different form. In addition, the representation is speculatively weaker than the concept: the representation is not aware of its own status nor does it see the form it gives to its own content. Therefore, the task of speculative philosophy is to transform representations into thoughts. The first important thing Ferrarin notices is that a representation makes a kind of universal out of a sensible content. Every representation thus rises from an intuition which is internalized, so to say brought into the subject: a representation is always my representation, since it belongs to me. This means that the representation involves a negation of the 'outwardness' of being. This allows the author to spend some clear and sharp words on the importance of the Erinnerung and the freedom of the erinnerte from the givenness and the material presence of an object (pp. 145-147). This move from the outside is balanced by the opposite move of the concept which intuits itself into an external production that has been produced by the concept itself. The clearest example in this respect is language: names and words involve a relation of identity with their content, and the form of their production is essentially related to their content, to the being of the product. Spirit thus proceeds to its own external (in a different meaning than that of the 'outwardness' of the object of the representation) realization in the form of a second nature. Ferrarin shows how reason enters and permeates the same world it has been producing as if it were another nature, more precisely its own nature, that is its world. Now, if all our contents are chronologically first represented, then the object lies beyond the representation as an isolated item among other isolated items. The logic of representation is then aggregation. How should the concept turn this logic into a properly speculative (self-comprehending) one? Before answering, Ferrarin specifies that, in order to understand this change correctly, it is first necessary to understand that representation is already thought. But it is a merely formal thought, while speculative thought is meant to overcome the distinction between form and content. The difficulty is not replacing a form with another but rather to see that the concept takes its own form back as being inseparable from its formed content. Undoubtedly, in all objective concretions of the concept, the concept is restricted and 
limited by the medium at issue. And this is true even of language. By facing this problem, Ferrarin makes his account of Hegel's idea of critique more explicit. The speculative proposition implies a critical re-definition of ordinary language. Hegel aims at dissolving the copulative structure of judgment, in which the "I" is responsible for the connection between subject and predicate. For Hegel, the real problem is not the correctness of 'our' knowledge but rather the truth of the content. In other words, the distinctive mark of the speculative proposition does not lie in an alternative theory of language but in both the demonstration of language's limits and in a radical, critical and continuous renovation of language: the real problem of speculative proposition is the truth as the subject, the selfdevelopment of the concept. The identity between content and form thus entails the move of the concept in taking itself back from its own representative manifestations. Hegel's idea of critique rests on the activities of i) questioning all certainties and ii) proving that the truth is the only subject in all those manifestations we could ever face as apparently being external to truth itself and therefore false.

The topic of the last chapter is sufficiently significant and complex to deserve a separate volume. Such a complete analysis of Hegel's understanding of Kant's philosophy is an unicum in the current scholarship on both thinkers. From a methodological point of view, Ferrarin clearly believes that any philosophical interpretation has to prove its own grounds and authenticity. In the comparison at issue, the author thus aims at proving how and why Hegel's understanding of Kant's philosophy is at times inaccurate and inadequate. Therefore, some of Hegel's readings, which are based on oversimplification of Kant's reflection, cannot be uncritically accepted. The same is valid for many contemporary interpreters (for example McDowell) who take Hegel's philosophy as a radicalization of Kant. Ferrarin's general premise is that although Hegel's comments on Kant are interesting and insightful, there are some crucial points which are deeply problematic.

The first issue concerns the Kantian "I" which Hegel transforms into the quintessential Category while for Kant the "I" represents the functional ground of all categories. Furthermore, Ferrarin criticizes Hegel's interpretive reduction of self-consciousness to the finite "I". Hegel here disregards the Paralogisms which are directed at refusing the identity between a function and a substance, and he does not grasp Kant's distinction between reason and the individual "I". Two parallel topics are important at this point: the view according to which the Critique of Pure Reason merely contains a theory of knowledge and the confusion between reason and the "I think". For Kant, reason works through the "I" but cannot be reduced to this "I". Hegel's interpretive premises seem to be the following: First, Kant's dichotomies are seen as unsolved oppositions, while Kant strived to show the connection between the different elements of the critique of reason (intuition and concept, sensibility and understanding, reason and understanding, natural causality and freedom, etc.). Second, the reduction of Kant's account of knowledge to the connection between intuition and concept (overlooking that this synthesis is not paradigmatic) which is a moment of the critique of reason and not its main achievement. Third, Hegel takes transcendental idealism to be a subjectivism, thus taking a perspective on the result of Kant's theory as the premise of his own interpretation of Kant. In general, Hegel clearly distinguished and keep separated all those elements that Kant distinguishes as well, though for the opposite purpose of understanding their original unity or, at least, legitimating their connection. Ferrarin, who is an expert on Kant too, shows that the very idea of a priori synthesis, as the guiding thread of the three Critiques, ultimately escapes Hegel. And Ferrarin argues that this hiatus is present in many contemporary readings of Kant. Accordingly, the debate on Kant's philosophy ought to have focused more on the meaning of the Architectonic (insofar as this refers to the self-development of reason) and the role of the ideas, as well as on a revision of the distinction between the constitutive role of the understanding and the regulative role of reason than on the role of the "I". Ferrarin (p. 208) argues convincingly, that a priori synthesis is Kant's formula to describe reason's causal power in the world. However, this also makes clear why Hegel's account of reason cannot be assimilated to Kant's. Hegel would never see reason as a faculty among others, but rather as the impulse to be at home in the world and govern it. For Hegel, reason has the internal necessity of recognizing its own work in all finite determinations, instead of always being understood as what it seems to be in this or that specific context. Lastly, if Kant's idea of reason is that of a normative causality, Hegel's reason is causa sui, the substance which turns into a subject. The latter is the subject of its own development. It is ceaselessly 'becoming' itself thereby implying the selfdevelopment of the passive substance. 
The topics covered by this study are amongst the most crucial for understanding the general articulation of Hegel's philosophy. One of Ferrarin's most remarkable merits is that of having developed a very specific analysis of several particular issues without ever losing the general movement towards the demonstrandum. The result is a dense but vibrant and stimulating book which tries, as Hegel himself meant to do, to show how a healthy skepticism can lead us towards the true meaning of an issue beyond all comfortable certainties. Ferrarin's overall point is both thematic and methodological. This is evident in all critics of some instrumental readings of Hegel as well as in Hegel's own interpretation of Kant. Such an attitude proves how seriously the author takes Hegel's idea of critique. Moreover, it proves that it is both possible and necessary to learn also from the mistakes and oversights of other interpreters (including Hegel) apart from the great achievements of the giants of our philosophical tradition. This book does not merely illuminate many important elements of Kant and Hegel's philosophy and establish a dialogue between two authors who are often understood as incompatible; it is also able to prove the efficiency of its own method. This is a book which does not only teach philosophy, but also how to philosophize. In sum, this book deserves the attention of anyone interested in understanding both Hegel, Kant and their contemporary interpreters. 


\title{
Mario Caimi, Ileana Beade, José González Ríos,Macarena Marey, Fernando Moledo, Mariela Paolucci, Hernán Pringe y Marcos Thisted: Diccionario de la filosofía crítica kantiana, Buenos Aires, Colihue, 2017, 507 pp. ISBN 978-950-563-450-7
}

\author{
Miguel HersZenbaun ${ }^{1}$
}

El texto de referencia es el esfuerzo conjunto de un grupo de investigadores argentinos especializados en la filosofía de Kant y la filosofía moderna. El coordinador del proyecto es Mario Caimi, por muchos años profesor titular dela materia Historia de la Filosofía Moderna en la Facultad de Filosofía y Letras de la Universidad de Buenos Aires, hoy profesor consulto de dicha casa de estudios, e investigador superior del Consejo Nacional de Investigaciones Científicas, Experimentales y Técnicas (CONICET)

Los autores restantes son reconocidos profesores de diversas instituciones universitarias argentinas (Universidad de Buenos Aires y Universidad Nacional de Rosario) y en su mayoría investigadores del CONICET. Se trata de un grupo que reúne especialistas en diversas áreas de la filosofía de Kant y en filosofía moderna en general. Casi todos ellos pertenecen al Grupo de Estudios Kantianos, grupo de investigación radicado en el Instituto de Filosofía Dr. Alejandro Korn de la Facultad de Filosofía y Letras de la Universidad de Buenos Aires.

El mencionado libro es el resultado de más de diez años de investigación. Se trata de un diccionario enciclopédico que recorre las diversas áreas de la filosofía kantiana y ofrece una mirada profunda y abarcadora sobre ellas. Como explica su prólogo, el Diccionario toma en cuenta las reconocidas obras de Mellin (Encyclopädisches Wörterbuch der kritischen Philosophie) y de Eisler (Kant Lexikon), pero no se trata de su traducción, sino que cada entrada es el resultado de la labor de los autores. Cabe mencionar que cada entrada va acompañada de su equivalente en alemán, latín, francés, italiano y portugués.

A fin de ejemplificar la profundidad y claridad del Diccionario, conviene detenerse en algunas de sus entradas.

Tomemos, por ejemplo, el término 'antinomia'. Los autores comienzan por indicar los múltiples sentidos que posee el término: en sentido objetivo, se refiere a la oposición de dos juicios que tienen una pretensión a priori de universalidad; en sentido subjetivo, se trata del estado de la razón del que, en busca de la totalidad absoluta, surgen raciocinios dialécticos que llevan a juicios contrapuestos. Estos juicios son los que conforman la antinomia en sentido objetivo. Los autores aclaran que el conflicto de estos juicios "es sólo aparente, pero esa apariencia de conflicto es inevitable" (p. 38).

Los autores señalan que contamos con tres especies de antinomia: la antinomia de la razón especulativa, la antinomia de la razón práctica y la antinomia de la razón con respecto a la facultad de juzgar. La antinomia de la razón especulativa se presenta bajo la forma de cuatro antinomias clasificadas según la tabla de las categorías. Estas se dividen en antinomias matemáticas (las dos primeras) y antinomias dinámicas (las dos últimas). La primera antinomia se refiere al origen y límites del mundo; la segunda se refiere a la divisibilidad o simplicidad de sus partes; la tercera se refiere a la posibilidad o imposibilidad de la causalidad por libertad; la cuarta se refiere a la existencia $o$ inexistencia de un ente necesario.

${ }^{1}$ CONICET - Universidad de Buenos Aires. 
Luego de presentar los conflictos cosmológicos de la Crítica dela razón pura, los autores pasan a una explicación sistemática de cómo son resueltos. En las cuatro antinomias, la contradicción entre los enunciados resulta irresoluble, si se sostiene que el mundo sensible es una cosa en sí. Por el contrario, una vez aceptado que el mundo es mero fenómeno, las contradicciones desaparecen. En el caso de las antinomias matemáticas, una vez aceptado que el mundo es mero fenómeno, se concluye que los juicios enfrentados son falsos. En el caso de las antinomias dinámicas, la distinción entre un mundo fenoménico y un mundo inteligible permite admitir que los pares de juicios son verdaderos, sólo que las tesis lo serán en relación con el mundo inteligible y las antítesis con el mundo sensible. La antinomia de la razón pura vale como una prueba indirecta de la idealidad transcendental de los fenómenos: si el mundo fuera una totalidad existente en sí, nos veríamos conducidos necesariamente a una contradicción; por lo tanto, debemos admitir que el mundo es fenoménico.

La antinomia de la razón práctica se presenta en las esferas de la ética y del derecho. La antinomia de la razón práctica en la esfera de la ética emerge del concepto de sumo bien. "El sumo bien es la síntesis necesaria de felicidad y virtud. Pero ni la apetencia de felicidad puede ser el móvil que nos lleve a buscar la virtud, ni la virtud es la causa eficiente de la felicidad, por lo que la unión de felicidad y virtud parece imposible" (p.39). Conforme a la distinción entre mundo sensible y mundo inteligible ha de encontrarse la solución de esta antinomia. La primera proposición - que la apetencia de la felicidad sea el móvil que nos conduce a la virtud - es falsa. La segunda proposición -i.e. que la virtud sea la causa de la felicidad-, en cambio, sólo es falsa cuando se la entiende como forma de la causalidad en el mundo sensible. Ahora bien, en tanto puedo pensarme como noúmeno en el mundo inteligible, puedo admitir, en este respecto, como posible que la intención moral tenga una conexión causal necesaria con la felicidad. Así, puedo pensar la posibilidad de la unión necesaria de virtud y felicidad en el mundo inteligible. Finalmente, en tanto estamos obligados a perseguir el sumo bien, debemos necesariamente suponer a la virtud como causa de la felicidad.

La antinomia de la facultad de juzgarse encuentra en el uso estético y en el uso teleológico de la facultad de juzgar. La primera enfrenta dos juicios: uno que sostiene que el juicio del gusto no se basa en conceptos y otro que afirma que el juicio del gusto se basa en conceptos. La solución consiste en admitir que ambos juicios son verdaderos, pero que el término 'concepto' se entiende en diferentes sentidos. La antinomia del uso teleológico de la facultad de juzgar presenta una contraposición entre un juicio que afirma que la producción de toda cosa material debe ser juzgada como posible por medio de leyes meramente mecánicas y otro juicio que sostiene que ciertos productos (los organismos) no pueden ser producidos sólo por medio de estas leyes. Los autores explican que se trata de un conflicto aparente, pues aquí no se atiende a la constitución de las cosas, sino al modo en que debemos juzgarlas. Así, todo fenómeno debe ser explicado según leyes mecánicas. Pero, a la vez, los organismos deben ser juzgados como si estuvieran bajo algún principio que actuase de acuerdo con causas finales.

Finalmente, los autores arriban a una antinomia de la razón práctico-jurídica sobre el concepto de lo propio y de lo ajeno. Esta antinomia enfrenta un juicio que sostiene que es posible tener algo externo, aunque no se esté en posesión actual de ello y otro juicio que sostiene que no se puede tener algo externo como propio si no se está en posesión de ello. La solución se halla en la distinción entre una posesión empírica (a la que se refiere la tesis) y una posesión inteligible (a la que se refiere la antítesis).

Pasemos al término 'razón'. A este término, de evidente importancia en la obra de Kant, se le dedican cinco páginas. Se lo estudia desde el punto de vista lógico, gnoseológico, transcendental, metafísico y antropológico. A su vez, se considera a la razón en su uso teórico como en su uso práctico.

En lo que refiere a su uso teórico (en el que la facultad determina su objeto), la razón en su uso lógico es la facultad de deducir lo particular de lo universal. Se trata de la facultad capaz de llevar a cabo raciocinios (silogismos) o inferencias mediatas. Desde el punto de vista gnoseológico y según este mismo uso teórico, se la concibe como facultad de los principios: llevar a cabo un silogismo es alcanzar un conocimiento a partir de otros conocimientos, utilizando la premisa mayor del silogismo como un principio para ello. Por medio de este procedimiento, la razón procura llevar los múltiples conocimientos del entendimiento a una unidad sistemática a priori, ordenando las múltiples reglas del entendimiento bajo la unidad de principios superiores. La representación de esta unidad se da bajo 
representaciones denominadas ideas, que no brindan principios constitutivos de objetos, sino principios meramente regulativos de la actividad del entendimiento. Cuando a estas representaciones se les atribuye validez objetiva (i.e. se las toma como principios constitutivos de objetos), se producen los raciocinios dialécticos estudiados en la 'dialéctica transcendental'. Aquí entramos en el terreno del uso transcendental (ilegítimo) de la razón, en tanto esta facultad es utilizada como facultad del conocimiento puro, que pretende ilegítimamente ampliar el conocimiento a priori más allá de los límites de la experiencia. Por medio de su uso lógico, a través de tres tipos de prosilogismo (silogismo ascendente), la razón nos conduce a tres representaciones de lo incondicionado o ideas: Alma, Mundo y Dios. Aquí se trata de la razón como facultad de las ideas o de la razón en su uso real, a diferencia del mero uso lógico formal. Estas ideas o representaciones de lo incondicionado no pueden tener un objeto correspondiente en la experiencia. Por esto, puede decirse que la razón, en sentido metafísico y como facultad de lo incondicionado, realiza un esfuerzo por obtener un conocimiento de lo suprasensible. A pesar de que el conocimiento teórico de lo incondicionado le está vedado, ella es capaz de un conocimiento teórico simbólico y analógico.

Pasemos a considerar la razón en su uso práctico. La razón es 'práctica' en tanto se refiere a su objeto para darle efectiva existencia. Se trata de la facultad que determina el hacer y el obrar. Es razón práctica 'técnica' cuando los determina según fines contingentes sensibles. Aquí la facultad brinda sólo imperativos condicionados, destinados a alcanzar fines contingentes. Si, por el contrario, la razón determina el querer y el obrar por medio de puros conceptos, se trata de la razón 'práctica pura'. Esta es la "facultad de determinar por medio de ideas (conceptos racionales puros) el uso libre de nuestra causalidad" (p. 403). A esto llama Kant en los seres humanos el principio de autonomía o de libertad.

La razón práctica pura contiene principios a priori constitutivos válidos para la facultad apetitiva, por medio de lo cual ella determina lo que debe ser. Es la razón práctica pura la fuente de una norma absoluta, que al hacérsenos consciente - a través de lo que se denomina factum de la razón, esto es, "un hecho suprasensible en el sujeto moral" (p. 403) - conocemos la causalidad suprasensible en el ser humano como ser racional (o causalidad por libertad). Esto nos ofrece un conocimiento práctico (no teórico) de un ente con causalidad no sensible y nos conduce a los postulados de la razón (libertad de la voluntad, inmortalidad del alma y existencia de Dios), cuya realidad objetiva es sólo práctica. Es por medio del imperativo categórico como legislación de la razón práctica pura como el ser humano se descubre como miembro legislador de un reino inteligible de los fines y como un fin en sí mismo. El objeto de la razón práctica pura es el bien supremo. La razón en su uso práctico prima sobre la razón en su uso teórico. Esto significa que la razón proporciona la determinación práctica de lo que se encuentra fuera del alcance del uso teórico de esta facultad, ocupando el concepto de 'libertad' un lugar preponderante en el sistema crítico.

La entrada del Diccionario concluye abordando la razón en sentido antropológico, indicando que aquí se llama razón a la facultad de concebir por uno mismo los principios del conocimiento y de la acción, a lo que se opone la 'incapacidad legal' o 'minoridad'.

Veamos el término 'transcendental'. Los autores comienzan indicando un sentido del término 'transcendental' no presente expresamente en los textos kantianos. Se dice de aquellos predicados que se pueden enunciar con verdad de manera necesaria y universal de toda cosa. En este sentido, se trataría de predicados que están más allá de todo género y toda clasificación. Luego de la Revolución Copernicana, para Kant no habría predicados que estén por encima de las categorías, en tanto todo objeto, para serlo, depende de una síntesis categorial. Así, las categorías, como condiciones de objetividad de todo objeto, pueden ser llamadas 'transcendentales'. De esta manera, explican los autores, "se llaman [...] transcendentales aquellos predicados que se pueden enunciar de manera necesaria y universal (es decir, a priori) de todos los objetos porque son condiciones de la objetividad misma" (p. 475).

En la filosofía kantiana, el término transcendental se aplica a cierto conocimiento de segundo orden, al conocimiento del conocimiento a priori. El conocimiento transcendental pretende explicar cómo es posible conocer a priori. Se trata de un conocimiento del conocimiento a priori y de cómo éste es posible. Se trata, así, de una "teoría del conocimiento" (p. 476) que se ocupa de "la crítica del conocimiento" (KrVB81; ápud p. 476). El conocimiento transcendental tiene por funciones propias fundamentar el conocimiento a priori y establecer el alcance y límite de las facultades 
cognoscitivas utilizadas en el conocimiento de objetos. También se califica de 'transcendental' a cada elemento identificado como condición a priori del conocimiento en general y a las condiciones del conocimiento a priori. Algo es transcendental si es fuente de fundamentación de la posibilidad de conocimiento a priori.

Los autores distinguen el conocimiento transcendental de las estructuras del pensamiento de la lógicaformal, del conocimiento transcendente, del conocimiento empírico y "del conocimiento a priori que no versa sobre otros conocimientos, sino sobre la sensibilidad o sobre objetos" (p. 476).

Primero, el conocimiento transcendental es un conocimiento del conocimiento, es decir, de pensamientos referidos a objetos, y se opone a las meras estructuras a priori del pensamiento construidas por la lógica formal (meros pensamientos no necesariamente referidos a objetos). La lógica deja de ser meramente formal y pasa a ser 'transcendental', cuando se ocupa de las reglas del pensamiento puro de objetos. Así, el término transcendental queda vinculado al 'conocer' y opuesto a lo lógico-formal, asociado al mero 'pensar'.

Segundo, los autores distinguen conocimiento transcendental de conocimiento transcendente (advirtiendo aquellos casos en que Kant utiliza el primer término en el sentido del segundo). El uso transcendental de conceptos se refiere al uso a priori respecto a objetos, el que sólo es legítimo en tanto se refiera a objetos dados en la sensibilidad, y no es legítimo si se pretende conocer a priori cosas en sí u objetos no sensibles, es decir, si se pretende conocer a priori objetos sin atenerse a las condiciones de la sensibilidad. Este uso transcendental ilegítimo es más exactamente un 'uso transcendente'. Ahora bien, "sólo la posibilidad de la experiencia sirve de fundamento de la validez del conocimiento transcendental" (p. 477), con lo cual todo uso de las categorías que no remita a la experiencia posible y a las condiciones formales de la sensibilidad (sólo dentro de cuyos límites puede ser dado el objeto al que refieren los conceptos puros del entendimiento) es ilegítimo y 'transcendente'. Es en este sentido como 'transcendental' termina por significar "relativo a la posibilidad de la experiencia" (KrVB 401, ápud p. 477).

Tercero, el conocimiento transcendental se relaciona con el conocimiento empírico, en tanto en éste se encuentran también elementos a priori. Es tarea del conocimiento transcendental explicar la posibilidad del conocimiento empírico, en tanto entre las condiciones de posibilidad del conocimiento empírico hay condiciones que valen a priori (i.e. con independencia de la experiencia).

Cuarto, en lo que se refiere a la relación entre conocimiento transcendental y otros conocimientos a priori, los autores explican que no todo conocimiento a priori es necesariamente transcendental. No todo conocimiento a priori — por ejemplo, la geometría- tiene por objeto otro conocimiento (no es conocimiento de la posibilidad del conocimiento a priori). "El conocimiento $a$ priori que se puede llamar transcendental es aquel por el cual conocemos que ciertas representaciones (intuiciones o conceptos) se pueden aplicar a priori a objetos de la experiencia [...] porque sirven de fundamento de la posibilidad de un conocimiento a priori" (p. 478).

Por otra parte, el término transcendental se refiere al origen del conocimiento, esto es, a las facultades que hacen posible el conocimiento a priori. En este sentido, la unidad sintética necesaria del múltiple fenoménico y la imaginación son transcendentales, en tanto sólo gracias a ellas se produce la experiencia. La síntesis transcendental no sólo tiene lugar a priori, sino que fundamenta otros conocimientos a priori.

Finalmente, el término transcendental se refiere a la filosofía transcendental, la cual es un sistema de todos los conocimientos transcendentales, cuya tarea es explicar cómo es posible el conocimiento a priori, i.e. explicar cómo son posibles los juicios sintéticos a priori (juicios a través de los cuales se vehiculiza dicho conocimiento). El examen de dicho conocimiento es la Crítica de la razón pura. "La filosofía transcendental es el sistema [...] de todos los conceptos y principios cuyo origen es a priori y que se refieren a objetos en general" (p. 479). Todo esto es asunto de la Crítica de la razón pura, que es la idea completa de la filosofía transcendental, pero no la ciencia misma, pues esta Crítica sólo alcanza la evaluación completa de los principios del conocimiento a priori. A esta Crítica también compete establecer los conceptos y principios de la filosofía transcendental, la explicación de su posibilidad y el trazado arquitectónico de todo su plan. 
El Diccionario recorre la totalidad de la obra kantiana y se ocupa de los términos científicamente relevantes y de otros de menor alcance; se detiene en un estudio sistemático y detallado de los conceptos fundamentales de la filosofía crítica, condensa lo fundamental de cada materia y lo expone de manera sistemática, ordenada, profunda y clara.

Es un libro de referencia obligatoria para especialistas en la obra de Kant, en el Idealismo alemán y en la filosofía moderna, así como para doctorandos y estudiantes de grado. Vale aclarar que se trata del primer diccionario kantiano en español, estableciéndose así como un excelente antecedente para la tradición de kantianos hispanoparlantes y que permanecerá en esta tradición como una referencia ineludible.

Celebro la aparición de este Diccionario y que venga de la mano de un grupo de especialistas en el que está muy bien representado el Grupo de Estudios Kantianos. 


\title{
Paula Órdenes Azúa y Daniela Alegría (eds.): Kant y el Criticismo: pasado, presente, y ifuturo?, Porto Alegre, Editora Fi, 2015, 303 pp. ISBN 978-85-66923-52-0
}

\author{
CONSTANZA TERRA ${ }^{1}$
}

Los días 7, 8 y 9 de abril de 2014 se celebró en Santiago de Chile el Primer Congreso Internacional: Kant y el Criticismo: pasado, presente y ifuturo?, evento que congregó a un considerable número de estudiosos del pensamiento kantiano, quienes presentaron sus trabajos a un numeroso público asistente. El evento resultó ser exitoso y, producto de lo anterior, las organizadoras llevaron a cabo una publicación que recogió algunas de las comunicaciones que se presentaron en el congreso. Mediante un criterio riguroso de selección, las editoras, Paula Órdenes Azúa y Daniela Alegría, establecieron una selección y edición de veinte artículos que componen, finalmente, el libro en cuestión. Las editoras se propusieron dos objetivos al momento de su selección que dan origen a esta publicación: i) reunir y exhibir el trabajo de investigación de una nueva generación de especialistas en los estudios kantianos, que - de ser posible - diera cuenta de 'nuevas formas de aproximación' al pensamiento de Kant y ii) reunir artículos que aborden, por una parte, algunos de los problemas clásicos que han acompañado a la filosofía kantiana, tales como epistemología, la filosofía práctica, la estética y la teleología mientras que, por otra, den cuenta de los posibles rendimientos que dicha filosofía puede tener a la luz de los problemas filosóficos más contemporáneos. Dicho esto, las editoras nos advierten, además, que si bien el título del libro es Kant y el criticismo, el criterio de selección de los artículos no sólo se vio restringido al período crítico de Kant, sino también a su filosofía pre-crítica, así como a lecturas futuras que puedan desprenderse del vasto pensamiento del filósofo de Königsberg. Por esta razón, parte del título que acompaña al ya señalado es tanto la alusión al pasado y su presente como alposible futuro de la filosofía crítica kantiana, así como al interés inagotable que suscita. Con el fin de hacerse cargo de estos objetivos y exigencias, las editoras dividen el libro en cuatro capítulos que corresponden, a su vez, a cuatro ejes temáticos fundamentales: aproximaciones epistemológicas en torno a Kant, consideraciones sobre la estética y teleología en la filosofía crítica, observaciones a partir de la filosofía moral de Kant, y enfoques prácticos del kantismo (filosofía política, filosofía del derecho y filosofía de la educación).

La primera parte del libro es, quizá, la menos relevante en la selección de textos con respecto al resto de la edición, puesto que los artículos seleccionados no significan mayor contribución al ámbito de la epistemología kantiana. Sin embargo, hay dos artículos que destacan en esta primera sección. En primer lugar, el artículo de Mariela Paolucci ("Sobre un argumento en torno a la posibilidad de otros mundos en el joven Kant") resulta interesante y novedoso al presentar un análisis acerca de la primera obra publicada por Kant Pensamientos sobre la verdadera estimación de las fuerzas vivas (Gedanken von der wahren Schätzung der lebendigen Kräfte). Esta obra no ha gozado de suficiente atención por parte de los estudios kantianos especializados, por lo que un artículo dedicado a ella no sólo es bienvenido, sino también agradecido por quienes no estamos familiarizados conel período pre-crítico más temprano. Por su parte, el artículo de Cristóbal Olivares ("Crítica kantiana del concepto de inconsciente y operación freudiana sobre la filosofía trascendental en la obra juvenil de Theodor Adorno") está dedicada a un tema poco explorado por los estudiosos del kantismo, aunque por lo mismo corre el riesgo de alejarse del tema en cuestión propiamente tal — pero ahí también estriba lo interesante y novedoso de su propuesta-.

El segundo capítulo del libro es mucho más interesante en la selección de artículos y presenta una mayor contribución al ámbito del saber que señala (estética y teleología). En general, la selección es bastante sobresaliente. Destaca, particularmente, el artículo de Luciana Martínez ("Una

\footnotetext{
${ }^{1}$ Universidad Diego Portales - Universidad de Leiden.
} 
función del genio en la Deducción del principio a priori de los juicios de gusto"), que da luces sobre una de las funciones del genio dentro de la crítica de la facultad de juzgar estética. Si bien su propuesta puede resultar modesta y restringida en un inicio, su artículo resalta por la claridad de la exposición y por mostrar la facilidad de la autora para iluminar ciertos pasajes de la tercera Crítica que son particularmente difíciles y oscuros. Otro artículo interesante es el de una de las editoras del volumen, Paula Órdenes Azúa ("La antinomia de la facultad de juzgar y el rol del entendimiento intuitivo en su resolución"), que aborda uno de los problemas que, sin duda, más interés y discusión ha generado dentro de la recepción de la crítica de la facultad teleológica, a saber, la antinomia entre mecanismo y teleología. La autora acota el tema de la antinomia a dos problemas fundamentales: cómo se posibilitaría una antinomia de la facultad de juzgar teleológica, y qué rol jugaría el entendimiento intuitivo en la resolución de dicha antinomia. Además, este artículo presenta una explicación clara e interesante acerca de cómo debemos entender los conceptos de mecanismo y teleología en dicho contexto (asunto que está lejos de ser claro en esos pasajes escritos por Kant y que, por lo mismo, ha causado un desfile de interpretaciones y comentarios tan diversos como contradictorios entre sí), lo que le facilitaría tremendamente la lectura a un posible lector entrampado en esos difíciles pasajes.

En el tercer capítulo la selección de los artículos es más equilibrada, ya que cada uno de ellos aborda perspectivas diversas sobre la filosofía moral de Kant y sus problemas - a esta alturaya clásicos. Hay varios artículos a destacar que podrían representar una contribución significativa a los estudios de la filosofía práctica kantiana (tarea ardua si se considera el gran número de publicaciones que año a año surgen en torno al tema). Por ejemplo, sobresalen los artículos de Harald Bluhm ("Opinar, saber, creer: los modos del tener por verdad y la razón ajena a Kant"), Susan V. H. Castro ("How to Treat Humanity: Autism and Moral Imagination") y Daniela Alegría ("Kant y Mill. El problema de la imparcialidad en la modernidad"), ya sea porque extienden la filosofía kantiana más allá de Kant y su concepción práctica-moral o porque vinculan la filosofía moral kantiana a problemas de la filosofía práctica reciente.

El último capítulo del libro es, tal vez, el más interesante y novedoso con respecto al diálogo de temas kantianos con otras esferas del saber, como lo es el ámbito político y educacional. En este sentido, sobresale el artículo de Nicolás Ried ("La cuestión del derecho a la revolución en Kant. Elemento para una genealogía de lo político") que hace, a partir de la afirmación de Kant de que el derecho a la revolución es eminentemente ilegítimo, una lectura sobre la revolución como forma de violencia que, en última instancia, no pretende legitimarse y que presenta una nueva forma de pensar lo político - cuestión que entra en tensión, a su vez, con la propuesta kantiana-. El texto de Rosa Viviana López Ortega ("Proyecto educativo de Immanuel Kant: crítica y formación de la humanidad") también representa una virtud especial en comparación con el resto de la selección: López ofrece un vínculo de la filosofía kantiana con el ámbito de la educación. Si bien este vínculo puede resultar evidente, la escisión profunda que existe entre el estudio académico kantiano y la labor pedagógica de la filosofía demuestra que esa obviedad se resiente en el quehacer académico, ensimismado y cerrado sobre sí mismo. Para solventar aquella escisión, la autora propone leer en la obra de Kant un llamado a establecer no sólo una filosofía crítica, sino también una 'educación crítica'.

El libro reseñado cumple, en suma, con los dos propósitos enunciados en un comienzo: mostrar las investigaciones y contribuciones de una nueva generación de estudiosos de la obra de Immanuel Kant y presentar un conjunto de reflexiones que no se restringen únicamentea su período crítico. Pese a ciertos altibajos en la selección de textos y en el cuidado de la edición de los mismos (falta de unificación de estilo de citación y existencia de erratas, por ejemplo), la presente publicación responde favorablemente a esa intuición inicial que señalan las editoras en el prólogo, a saber, que a la filosofía de Kant no sólo la ocupa un pasado y presente, sino también - y sobre todo - un futuro que parece estar lejos de agotarse. 


\title{
Juan Ormeño Karzulovic y Miguel Vatter (eds.): Forzados a ser libres. Kant y la teoría republicana del derecho. Santiago, Fondo de Cultura Económica, 2017, 243 pp. ISBN: 978-956-289-149-3.
}

\author{
CRistóBAl OLIVARES ${ }^{1}$
}

\begin{abstract}
Este libro editado por los profesores Juan Ormeño y Miguel Vatter pretende ser un aporte novedoso a los ya tradicionales estudios de filosofía práctica kantiana: "Los autores aquí reunidos representan la investigación más avanzada y reciente sobre el pensamiento político y jurídico de Kant” (p. 13). El libro en cuestión se divide en cuatro capítulos y reúne los ensayos de diez investigadores latinoamericanos y alemanes. En el primer capítulo, "Naturaleza y moral", se aborda la problemática que hay en torno al "papel desempeñado por la naturaleza humana en el derecho y la moral kantianos" (p. 14). El primer ensayo, perteneciente a Ernesto Garzón Valdés y que lleva por título "Siete pecados capitales kantianos", aborda una serie de "pecados político-morales" (p. 25) definidos como nocivos para el horizonte de un mundo cosmopolita (del "orden democrático nacional e internacional", dice el autor). Señala Garzón que el origen de estos 'pecados' radica en una concepción errónea de la naturaleza humana. Si se parte de una consideración 'angélica' del hombre (primer pecado), que motivado por su innata bondad natural marcharía inexorablemente de lo malo hacia lo bueno, se ha de suponer, entonces, que el orden civil implica hombres ya moralizados. A luz de los hechos históricos, la consideración angélica de la naturaleza humana se muestra tan utópica e irrealizable que, producto de este mismo 'pecado', se produce un movimiento en sentido inverso: desplazamiento casi inmediato hacia una consideración pesimista. Entonces, el orden civil se torna imposible de realizar, puesto que no hay ni habrá ciudadanos moralmente buenos. El último pecado consiste en la resolución de los conflictos nacionales a internacionales a través de la guerra. Esta última, afirma Garzón, viene a ser el pecado político-moral de la caída del hombre en la "animalización" (p. 45). Este séptimo pecado se corresponde bien con la consideración de la 'naturaleza angélica' del hombre, que describe Garzón, puesto que depende de un cierto pesimismo frente a la razón y el devenir humano. Hay guerra, es decir, hay pecado, porque la paz se vuelve imposible.
\end{abstract}

Más allá del problema de la angelización y animalización, Juan Manuel Garrido en su ensayo "¿Y si la vida no fuera un derecho? Ser vivo e ius necessitatis" aborda una concepción de la vida bastante distinta a la contenida bajo el concepto de "naturaleza humana". En mi opinión, y sin que Garrido lo explicite así, el aporte de Kant al derecho moderno radicaría en la desconstrucción del 'derecho de necesidad' por el que se definía tradicionalmente la idea de ser vivo, al diferenciar los derechos de la persona humana de lo que propiamente puede considerarse como una 'vida'. La tesis de Garrido es que "en sí misma y por sí misma, la vida no tiene derechos; solo la persona puede ser sujeto de derecho y de propiedad" (p. 47).

El capítulo segundo, "Derecho y moral", se compone de tres ensayos que se enfocan en "[1]a distancia que separa la teoría moral kantiana de su pensamiento jurídico" (p. 16). Encontramos aquí el aporte dos importantes profesores alemanes ("¿Qué es erróneo en una interpretación moral de la filosofía política de Kant?" de Christoph Horn y "Derecho y coacción. ¿Puede derivarse la concepción del derecho de Kant de su teoría moral?" de Marcus Willascheck) y el ensayo del joven investigador Nicolás Vargas Carlier, titulado "A propósito de finalidad y formalidad en la formulación del imperativo categórico". Los trabajos de Horn y Willascheck se complementan bastante bien al documentar y argumentar las razones de la brecha insalvable entre la filosofía política y jurídica de Kant respecto a su previa elaboración ético-moral. En dirección contraria, Nicolás

\footnotetext{
${ }^{1}$ Pontificia Universidad Católica de Chile - Universidad Complutense de Madrid.
} 
Vargas desarrolla las huellas de una proximidad entre el imperativo categórico y el deseo de un mundo social imaginado en la "Doctrina del derecho" de la Metafisica de las costumbres.

En el capítulo tercero, "La libertad del derecho", encontramos lo que, a mi modo de ver, son los tres artículos centrales de Forzados a ser libres, no tanto por el aporte, indudable, a la "teoría republicana del derecho' de Kant como por el rol articulador que cumple este capítulo en el libro en cuestión. El ensayo "Libertad y exterioridad" de Eduardo Molina Cantó es un pedagógico texto que demuestra, con suficientes argumentos y documentación, la diferencia radical que para Kant posee el punto de vista jurídico y el punto de vista moral. Esta diferencia se explicará a partir de la pluralidad de sentidos que la palabra 'libertad' tiene en la obra de Kant (Molina, siguiendo a Lewis White Beck, distingue cinco sentidos de libertad). El autor desarrolla, especialmente, la diferencia entre una 'libertad interior' y otra 'libertad exterior', y su articulación correspondiente en La metafisica de las costumbres. El ensayo de Molina sirve como introducción a los trabajos de Ormeño y Vatter, en el sentido que estos "se centran en el deber jurídico de respetar la libertad exterior de las personas" ( $p$. 18). En un tono muy hegeliano, Juan Ormeño, en "Violencia y autorrespeto: las bases kantianas de la libertad exterior", quiere demostrar que "la existencia de un estado civil" es condición de posibilidad del "derecho innato de libertad" (p. 19). En otras palabras, según Ormeño, no habría una realización práctica de la libertad humana si no se sostuviera sobre los cimientos jurídicos y políticos de un Estado. El autor problematiza la manera por la cual es posible conciliar este 'derecho innato de libertad' con la coacción propia de todo 'estado civil'. Miguel Vatter, en cambio, en "El derecho a tener derechos y las reglas de Ulpiano en la Doctrina del derecho kantiana" va a desarrollar una suerte de interpretación arendtiana de Kant en lo que refiere al "derecho a la libertad exterior" (p. 175). Lo que Vatter realiza es una lectura arendtiana de la filosofía del derecho de Kant, asociando la operación del juicio reflexionante de la Crítica de la facultad de juzgar (que para Hannah Arendt es la fuente del auténtico pensamiento político kantiano) con el "derecho innato" a la "libertad externa" (p. 181) de la Doctrina del derecho de La metafísica de las costumbres. En la medida que este derecho innato es concebido por Vatter como el verdadero poder constituyente, distinto del poder constituido encarnado por el Estado y sus representantes, el autor va a producir una cadena en la que el poder constituyente (o derecho innato a la libertad externa) se va a articular con el pueblo de ciudadanos a través del juicio reflexionante. Esta independencia del 'derecho a la libertad externa', que implica una brecha entre pueblo y soberanía, o entre ciudadanos y Estado, resulta finalmente en que no se pueda concebir la 'libertad exterior' como mero producto y determinación obrada por el Estado, sino que esta forma de libertad ha de concebirse como una reserva de poder popular no-soberano, listo para actuar como espectador vigilante, juez y crítico de las instituciones soberanas ya constituidas.

Por último, en el capítulo cuarto, titulado "Moral, historia, cosmopolitismo", nos encontramos con la problemática entre derecho público y privado, entre Estado y propiedad. Nos dicen los editores que los ensayos de Alessandro Pinzani ("Justificación normativa y justificación funcional de la necesidad del Estado en Kant") y Daniel Loewe ("Libertad y propiedad en la fundamentación del Estado y el cosmopolitismo kantiano") tienen en común considerar que "el horizonte último de la teoría política y jurídica kantiana es el horizonte cosmopolita de La paz perpetua" (p. 20). En el caso del ensayo de Pinzani, se trata de encontrar una justificación intermedia entre lo jurídico y lo histórico de "la necesidad de salir del estado de naturaleza para entrar en el estado civil" (p. 197). El trabajo de Loewe sostiene que las consecuencias últimas del cosmopolitismo, a diferencia de las tesis planteadas explícitamente por Kant sobre este punto, se dirigen a la constitución de una 'República Mundial de Estados' o 'República Mundial de Ciudadanos del Mundo'.

Ha de destacarse que los trabajos que el lector podrá encontrar en este libro no han sido organizados en torno a los ejes usualmente privilegiados por los comentaristas (me refiero a los ejes Fundamentación de la metafísica de las costumbres y Crítica de la razón práctica, que operan más bien en el registro ético-moral), sino que, como ha sido visto, se agrupan en torno a una de las ultimísimas etapas de la filosofía práctica kantiana: la Metafisica de las costumbres, específicamente a las cuestiones que surgen de la lectura de su Doctrina del Derecho. La Metafisica de las costumbres es una obra que, tal como señalan Ormeño y Vatter en su introducción, ha comenzado a tomarse en cuenta con la suficiente seriedad recién a partir de la segunda mitad del siglo XX. Valdría la pena detenerse un momento en las razones que los profesores Ormeño y Vatter consideran que han llevado a encumbrar, actualmente, a la Metafisica de las costumbres como uno de los clásicos de la filosofía 
política y del derecho para entender, entonces, la relevancia que Forzados a ser libres puede tener para los lectores del mundo hispanoamericano.

Tal como relatan los editores en la introducción, el orden mundial que nace tras el fin de la Segunda Guerra Mundial trae consigo una serie de mutaciones en política y derecho internacionales. Antes de estos acontecimientos, el pensamiento político-jurídico de Kant se encontraba más bien opacado por las reflexiones políticas canónicas. En este contexto, podría datarse el año 1971, año de la publicación de Un teoría de la justicia de John Rawls, como uno de los hitos de la segunda mitad del siglo XX, por el cual comenzará a destacar un nuevo enfoque - más o menos inédito hasta ese momento- de la filosofía kantiana: "[a]ntes de la publicación de Una teoría de la justicia de John Rawls, Kant no era considerado un pensador político fundamental que estuviese a la altura de un Maquiavelo o de un Hobbes, de un Hegel o de un Marx" (p. 11). Es a partir de las discusiones que generó la obra de Rawls que será posible, desde entonces, hablar de un interés propiamente jurídico de la filosofía kantiana. Los editores destacan especialmente la década final del siglo XX, tras la caída del Muro de Berlín, momento en el que Europa se reunifica tomando, una vez más, las banderas del derecho cosmopolita: "[c]uando, después de la caída del Muro de Berlín, se derrumbó el orden mundial de la Guerra Fría y se comenzó a entrever un nuevo orden normativo global —rápidamente formulado en los esbozos del derecho de gentes por parte del propio Rawls, de Habermas y de otros-, la apuesta de Kant por un destino cosmopolita para el derecho público europeo lo elevó a las alturas, casi, de un nuevo profeta de la política" (p. 11-12).

En cierto modo, Forzados a ser libres viene a ser una reflexión en lengua española de un episodio de la historia jurídico-política europea. En esta descripción se concentran tanto las virtudes como los defectos de este libro. Porque no cabe duda del enorme aporte que Forzados a ser libres significa al desarrollo de la filosofía práctica kantiana entre la comunidad de especialistas en lengua española. Sin excepciones - y el lector podrá evaluarlo por sí mismo-, encontraremos en este libro trabajos de profundo análisis, muy bien documentados, que atienden a las discusiones más desarrolladas en torno a la filosofía del derecho de Kant (véase el segundo capítulo, por ejemplo). En efecto, el mundo hispanoamericano se encontraba en deuda en lo que refiere a la reflexión sobre la 'teoría republicana del derecho' de tipo kantiano y uno no puede más que saludar el esfuerzo que han puesto los autores en la publicación de este texto. Pero, a mi juicio, se echa de menos algo así como una toma de posición de los autores en general, y de los editores en específico, frente al populismo latinoamericano y europeo, sobre todo si uno de los objetivos del libro era presentar "el carácter y los problemas fundamentales del pensamiento político kantiano en su relación con los debates de la actualidad" (p. 13). Ormeño y Vatter omiten, por lo menos explícitamente, la necesidad y la urgencia de replantear la 'teoría republicana del derecho' de Kant en confrontación con la poderosa tendencia de restitución del paradigma soberanista en política y derecho internacionales. Desde hace algunos años, quizá hace ya una década, hemos sido testigos de la desaparición de aquellas condiciones históricas que habían permitido que el pensamiento político-jurídico de Kant cobrara vigencia. Hoy el mundo es menos cosmopolita que hace diez años. Que el lector juzgue si los ensayos del capítulo cuatro de Forzados a ser libres han tomado nota de estas transformaciones. Relanzar hoy la investigación de un texto como Metafísica de las costumbres no debería omitir las delicadas turbulencias de la historia más 'avanzada y reciente' del siglo XXI americano y europeo. No obstante, es deseable que este libro cuente, por sus propios méritos, con buena recepción entre los especialistas de Kant. 


\title{
Immanuel Kant (ed. y trad. Gonzalo Serrano): La Deducción trascendental y sus inéditos, 1772-1788. Bogotá, Universidad Nacional de Colombia, 2014, 321 pp. ISBN: 978-95-876-195-39.
}

\author{
PEDRO STEPANENKO
}

\begin{abstract}
Muy probablemente la "Deducción trascendental de los conceptos puros del entendimiento" sea la parte más estudiada de la Crítica de la razón pura. Quizá la "Estética Trascendental" compita con ella, pero, a diferencia de esta última, la estimación de la Deducción no ha disminuido a causa de los cambios científicos. Su reconstrucción ha excitado la reflexión de muchas generaciones de filósofos. El caso más asombroso es la obra en tres tomos del belga Herman J. de Vleeschauwer titulada de manera sencilla y objetiva La déduction transcendantale dans l'œuvre de Kant, publicada entre 1934 y 1937. En ella, el autor estudia la Deducción trascendental desde sus orígenes, en la caracterización de las representaciones intelectuales de la Dissertatio de 1770, hasta el Opus postumum, analizando cuidadosamente, por supuesto, las diferencias entre las dos versiones publicadas, la de la primera y la de la segunda edición de la Crítica de la razón pura.
\end{abstract}

La importancia de esta pieza clave de la primera Crítica es incuestionable. En ella, Kant pone en juego la posibilidad de que una estructura conceptual a priori condicione nuestra experiencia consciente y de esto depende, para él, la legitimidad de la metafísica. Pero además de esta importancia, las posibles trayectorias que se pueden trazar al intentar reconstruir su gestación, tomando en cuenta las distintas etapas en el desarrollo del pensamiento de Kant, han dado pie a una serie de investigaciones que en términos de Norman Kemp Smith constituyen "un excelente ejemplo de genio detective en el medio académico" (Kemp Smith 1923, 202). En efecto, a partir de la publicación que llevó a cabo Theodor Haering en 1910 de los Manuscritos de Duisburg y de la publicación de las Reflexiones sobre lógica y metafísica en los tomos XVI, XVII y XVIII de Kant's gesammelte Schriften (AA), editados por Erich Adickes, la investigación del origen y la transformación de la Deducción trascendental se ha convertido en un importante capítulo de los estudios de la filosofía teórica de Kant. Esta investigación comprende la catalogación y la datación de manuscritos, el análisis de la caligrafía y la tinta, la edición de las obras utilizadas por Kant en sus clases de lógica y metafísica, así como el estudio de las influencias que cambiaron el rumbo de la filosofía de Kant.

Las obras que utilizaba Kant en sus cursos de lógica y metafísica en la Universidad de Königsberg y en las cuales, a modo de anotaciones marginales, se encuentran muchas de las reflexiones que conforman los manuscritos póstumos de esta etapa de gestación de la Crítica de la razón pura son la Metaphysica de Alexandri Gottlieb Baumgarten y el Auszug aus der Vernunftlehre de Georg Friedrich Meier. Con respecto a las influencias en esta etapa de gestación, además de la importante influencia del matemático y astrónomo Johann Heinrich Lambert al inicio del planeamiento del problema, destaca, por los cambios incorporados en los últimos meses previos a la conclusión de la obra, la filosofía empirista de Johann Nicolas Tetens, expuesta en sus Philosophische Versuche über die Menschliche Natur und ihre Entwicklung, los cuales, de acuerdo con lo que Hamann le escribe a Herder en mayo de 1779, Kant tenía delante de sí (v. Carl 1989: 115). Suponemos que en su escritorio.

Kant le envió su Dissertatio a Lambert el mismo año de su publicación, en 1770, y mantuvo correspondencia con él. En ella, Lambert le recomienda aplicar a los fenómenos los conceptos intelectuales que Kant había caracterizado en la Dissertatio solo negativamente, como no provenientes de la experiencia y, por lo tanto, válidos para las cosas tal como son y no solo como nos aparecen. Esta recomendación pudo haber impulsado el giro definitivo hacia el planteamiento de la

${ }^{1}$ Instituto de Investigaciones Filosóficas, Universidad Nacional Autónoma de México. 
Deducción trascendental, tal como Kant lo expresó dos años después, en la famosa carta a Marcus Herz de febrero de 1772: ¿cómo es posible que conceptos que no provienen de la experiencia puedan, y más aún deban, aplicarse a los objetos de la experiencia? Los conceptos puros del entendimiento dejarán de ser desde este momento representaciones de algo no sensible para convertirse en elementos que articulan representaciones sensibles en la conformación de los objetos de la experiencia, dejarán de ser considerados, pues, como representaciones de cosas distintas a los objetos de la experiencia, para convertirse en piezas fundamentales de la concepción de un mundo objetivo de fenómenos del cual tenemos conocimiento sintético a priori. De Johannes N. Tetens, Kant adoptó la tarea fundamental que le otorga a la imaginación en la conformación de ese mundo; es decir, la visión de la imaginación como una facultad no solo reproductiva sino también activa que condiciona la receptividad de la sensibilidad, como una facultad mediadora entre el entendimiento y la sensibilidad.

En cuanto a la datación de los manuscritos y anotaciones previas a la primera edición de la Crítica de la razón pura, además del análisis de la caligrafía y la tinta, cabe destacar que Kant, como muchos de nosotros, usaba para escribir la parte de atrás de cartas y notas que recibía. De acuerdo con Adickes, editor de estos manuscritos en la edición de la Academia de las Ciencias, Kant tenía la costumbre de hacer esto poco después de haberlas recibido (AA XVIII, 269). Este hecho representó para Adickes uno de los hilos de la "muy compleja urdimbre" (AA XVII, IX) en que consiste el sistema de datación de las notas manuscritas de Kant. Tomando en cuenta que esto es solo uno de los hilos, que esa costumbre pudo no haber existido y que los demás hilos son igualmente cuestionables, podemos imaginar el complejo rompecabezas en el que se convierte la ordenación de estos manuscritos y las múltiples maneras de contar la historia de la gestación de la Deducción trascendental. Solo una especie de fascinación u obsesión pudo mantener la voluntad de aclarar esta trayectoria, la cual ha sido fomentada, claro está, por las propias declaraciones de Kant acerca de la Deducción y por los más de diez años previos a la publicación de la Crítica de la razón pura, en los cuales Kant no publicó nada.

En relación con lo que estaba pasando en esta 'década silenciosa', Kant le escribe a Herz en noviembre de 1776 y le dice que nunca se había encontrado tan "sistemática y sostenidamente ocupado" como en esos seis años anteriores, que espera obtener un "mérito duradero" con el material que prepara, el cual se acumula en sus manos como el agua en una presa (AA X, 198-199). Menciona también algunos obstáculos que ha superado en el verano anterior, refiriéndose muy probablemente a los asuntos que aborda en el Manuscrito de Duisburg, asuntos que competen a la Deducción. En cuanto a las observaciones acerca de esta sección central de la Crítica de la razón pura, Kant sostiene en el prólogo de 1781 que no conoce "investigaciones que sean más importantes para la indagación de la facultad que llamamos entendimiento" (A XVI, tr. M. Caimi) y en la propia Deducción (al inicio de la sección segunda) sostiene que no solo se trata de la justificación de la objetividad de las categorías en cuanto condiciones de posibilidad del pensar objetos de la experiencia, sino también de la posibilidad misma de que el entendimiento se refiera a objetos (A 97). En términos contemporáneos, Kant sostiene aquí que la Deducción ofrece una teoría de la intencionalidad, necesaria para la metafísica.

Semejantes afirmaciones no podían dejar de excitar la curiosidad de muchos académicos que durante muchos años han tratado de reconstruir o entender cómo llegó Kant a las ideas que presenta en la Deducción trascendental y cómo se siguen unas de otras. Hans Vaihinger y Norman Kemp Smith consideran que en realidad no hay continuidad en el texto de la Deducción, al menos en la primera edición, y que esa es la única manera de entender la incompatibilidad de líneas argumentativas. Y es que, de acuerdo con su explicación, Kant juntó fragmentos de notas redactadas a lo largo de los 'años silenciosos', en los cuales hubo cambios muy importantes con respecto no solo a las estrategias argumentativas, sino incluso a los objetivos mismos de la Deducción, como lo ponen en evidencia las propias declaraciones de Kant posteriores a la publicación de la Crítica de la razón pura. Hans Vaihinger y Kemp Smith dedicaron enormes esfuerzos a reconocer la época a la que corresponde cada párrafo de la Deducción y el panorama que obtuvieron no es el de distintos bloques correspondientes a distintas etapas, sino el de un verdadero mosaico en donde se alternan fragmentos de distintas épocas. Este tipo de reconstrucción lo que pretende es distinguir argumentos y proyectos. Pero deja inconclusa la tarea, ya que finalmente uno debe también preguntarse por qué Kant compuso el texto final de esa manera. Es cierto que la composición de la Crítica de la razón pura en general fue precipitada, como Kant mismo se lo confiesa a Christian Garve en agosto 1783: en cuatro o cinco 
meses juntó el material que "había pensado cuidadosamente a lo largo de 12 años"; cuando una exposición clara hubiera requerido varios años (AA X, 338).Sin embargo, el esfuerzo de proporcionar un argumento que logre hacer intervenir el mayor número posible de elementos comprendidos en el texto original no debe abandonarse ni ser sustituido por la escrupulosa distinción de diferentes líneas argumentativas. En el balance de estas dos cosas descansa una buena interpretación, como la que nos ofrece Wolfgang Carl en Der schweigende Kant. Pero las variables son tantas que ese material que heredamos de Kant nos deja abierto un campo enorme de posibles interpretaciones.

A pesar del rompecabezas en el que puede convertirse la reconstrucción de la evolución del pensamiento de Kant previo a la primera edición de la Crítica de la razón pura, contamos ya con una serie de textos que representan las principales estaciones de ese camino y que la mayoría de los estudiosos de la filosofía teórica de Kant acepta. Esas estaciones las recoge Gonzalo Serrano en $\mathrm{La}$ Deducción trascendental y sus inéditos, 1772-1788. Se trata de la carta a Marcus Herz de febrero 1772 , en la que se encuentran por primera vez las dos principales preguntas de la Deducción trascendental: "¿en qué fundamento descansa la relación con el objeto de aquello que en nosotros se llama representación?” y "¿cómo es posible una representación que se refiera a un objeto sin ser afectada de manera alguna por él?" (AA X, 130-131, tr. G Serrano), es decir, cómo es posible referirnos a objetos y cómo es posible que las representaciones que nos permiten hacer esto no hayan sido obtenidas de la experiencia. Se trata también de una selección de Reflexiones fechadas entre 1772 y 1775, en las cuales Kant intenta responder a la segunda de estas preguntas en términos de condiciones de posibilidad de los objetos de la experiencia, apelando al 'uso real' (metafísico en contraste con el meramente lógico) del entendimiento. La tercera estación de este recorrido lo constituye el Manuscrito de Duisburg, en el cual Kant hace intervenir por primera vez a la autoconciencia en la respuesta a la primera de las preguntas antes mencionadas, esto es, en la explicación de la intencionalidad y la objetividad de nuestras representaciones. La cuarta es el fragmento B 12, al parecer escrito bajo la influencia de Tetens, poco tiempo antes de la conclusión de la Crítica de la razón pura. En este manuscrito, Kant aborda la imaginación como una facultad imprescindible que media entre la sensibilidad y el entendimiento en la conformación de la experiencia. La propia Deducción en la versión de la primera edición de la Crítica de la razón pura completa la primera mitad de esta compilación.

Contar con esta serie de textos en un solo volumen y en una edición bilingüe alemánespañol es, sin lugar a dudas, un privilegio que será copiosamente aplaudido por todos aquellos académicos y estudiantes hispanoamericanos interesados en esta pieza clave de la filosofía occidental. También será un buen estímulo para fomentar su estudio en el medio hispanoamericano, siempre y cuando logremos resolver el problema de la distribución de los libros publicados por editoriales universitarias; en ese medio en el cual existen ya contribuciones importantes al análisis de la Deducción trascendental y de su gestación. Pienso en Kant: Estudio sobre los fundamentos de la filosofia crítica de Roberto Torretti (1967), cuya segunda parte (más de 250 páginas) está dedicada a la deducción; también en La formación de la Critica de la razón pura de José Luis Villacañas (1980) que, a pesar de no estar enfocado solo a la gestación de la deducción, analiza documentos clave de ésta última, como la carta a Marcus Herz de 1772 o el Manuscrito de Duisburg. Asimismo, cabe mencionar Categorías y autoconciencia en Kant: Antecedentes y objetivos de la deducción trascendental de las categorías de Pedro Stepanenko (2000), Leçons sur Kant. La déduction transcendantale dans la deuxième édition de la Critique de la raison pure de Mario Caimi (2007) y Los años silenciosos de Kant de Fernando Moledo (2014).

Traducir filosofía es una actividad complicada y fascinante al mismo tiempo. No solo se requiere reconocer el significado de las palabras, sino que es necesario interpretar lo que quiso decir el autor para proponer una manera de entender el texto. A pesar de todo este esfuerzo, siempre habrá alguien que no esté de acuerdo con tu traducción. Por ello, es bueno tener muchas versiones de un mismo texto. Pero traducir manuscritos es aún más complicado. Las complicaciones empiezan desde las posibles lecturas de las grafías del autor y puede suceder que una letra cambie el significado y la relevancia de las oraciones. El día en que se presentó la compilación de Gonzalo Serrano en la Casa Universitaria del Libro en la Ciudad de México, el 7 de septiembre de 2016, Fernando Moledo ilustró estas posibles complicaciones con un excelente ejemplo extraído del legajo de Duisburg. Se trata de una oración en la que Reicke y Haering leyeron en una grafía la conjunción alemana oder, mientras que Adickes leyó en ella un artículo femenino declinado en genitivo: der. Como puede verse, la 
diferencia es de una letra y las expresiones resultantes son estas: "sustancia, causa o composición" y "sustancia, causa de la composición". ${ }^{2}$ Para un metafísico kantiano que estudia la transición entre la Dissertatio y la Crítica de la razón pura esta diferencia puede tener graves consecuencias.

Aventurarse a traducir las reflexiones que Serrano nos presenta en esta compilación es, pues, un asunto de mucho mérito. Ofrecer una nueva traducción de la Deducción en ambas ediciones es también algo muy valioso, en virtud de las múltiples interpretaciones que se pueden ofrecer. Lo que me parece extraño es el tratamiento que Serrano le ha dado a la primera sección de la Deducción trascendental. Esta sección consta de dos parágrafos. En el § 13, Kant introduce la deducción con la célebre diferencia entre la quid juris y la quid facti, sostiene que contar la historia de cómo se han adquirido conceptos como el de sustancia o causalidad a partir de la experiencia no permite legitimar la necesidad y la universalidad que exigen; también sostiene que una deducción de esos conceptos es imprescindible para la metafísica y que los fenómenos "podrían sernos dados sin que nuestro entendimiento los encontrara conformes a sus categorías" (paráfrasis de A89-A91), siendo esta última afirmación una de las más controvertidas y discutidas de la deducción. En el § 14, Kant introduce la idea de las conceptos puros del entendimiento como condiciones a priori de la posibilidad de pensar objetos de la experiencia y de acuerdo con el prólogo a la primera edición de la Crítica es aquí donde se encuentra la deducción objetiva de esos conceptos. Serrano no incluye el $\S 13$ en su traducción y al $\S 14$ lo coloca, junto con un fragmento de ese prólogo, como un anexo de las secciones segunda y tercera. Es cierto que el argumento a favor de la necesidad o de la objetividad de los conceptos puros del entendimiento, así como las razones que justifican su uso en la experiencia, se encuentran en estas últimas secciones. Pero si la compilación contiene incluso la nota del prólogo a los Primeros principios de la ciencia de la naturaleza en la que Kant distingue dos objetivos de la deducción, no veo por qué la sección introductoria de la deducción en la propia Crítica no deba incluirse. Creo que esta ausencia obliga al compilador y a la casa editorial a ofrecer una segunda edición de este excelente libro en la que se subsane esta falta.

La segunda mitad de esta compilación está conformada por textos que anteceden la nueva versión de la Deducción que Kant presenta en la segunda edición de la Crítica de la razón pura y por la propia Deducción en esta segunda versión. Y es que la Deducción trascendental, a pesar de lo que hubiera querido Kant y de lo que a veces sostiene, más que una pieza de un andamiaje ya terminado, es un proyecto en desarrollo, como bien lo señala Gonzalo Serrano en su introducción (p. 15). Un proyecto que cambió significativamente para el propio Kant, como lo dejan ver las distintas e incluso incompatibles declaraciones que hizo después de la primera edición, declaraciones acerca de los objetivos y las partes prescindibles e imprescindibles de la deducción. Me refiero a las afirmaciones de la nota de los Primeros principios de la ciencia de la naturaleza y del fragmento del ensayo Sobre el uso de principios teleológicos en la filosofía, ambos incluidos en la compilación de Serrano.

Aunque Kant sostiene en la nota mencionada que la diferencia de una nueva versión de la deducción solo tendría que ver con la exposición y que la oscuridad que padece la primera edición se debe "a la habitual suerte que corre el entendimiento durante sus investigaciones, en las cuales normalmente el camino más corto no es el primero del cual se percata" (AA IV, 475-476), lo cierto es que las dos versiones de la deducción han propiciado diferentes interpretaciones de la epistemología y de la filosofía de la mente kantianas. En la versión de la segunda destaca el aspecto normativo de los procesos de síntesis y la idea de un agente epistémico, poco presentes en la versión de la primera edición. Mientras que en la primera edición predomina la descripción de los procesos del entendimiento y de la imaginación mediante los cuales las categorías sintetizan intuiciones bajo la unidad de la conciencia y de esta manera conforman objetos de la experiencia, en la segunda edición destaca también un uso normativo de las categorías que presupone una concepción más robusta del yo que la presupuesta por la mera unidad de la conciencia. Esto sucede gracias a que Kant vuelve a enfatizar la función que desempeñan los juicios en la conformación de la experiencia, como lo hizo alrededor de 1772, y a la distinción entre juicios de percepción y juicios de experiencia que introduce en los Prolegomena, en la parte correspondiente a las deducciones metafísica y trascendental de la Crítica de la razón pura. Esta parte de los Prolegomena también la incluye Serrano en su compilación.

${ }^{2}$ Esta complicación en la lectura del Manuscrito de Duisburg la expone Fernando Moledo en la nota de la página 170 de su libro Los años silenciosos de Kant (2014). 
Dos secciones más completan esta compilación: una serie de reflexiones sobre metafísica que Kant escribió entre 1783 y 1786, y un fragmento de las lecciones de metafísica de 1784 y 1785 conforme a los apuntes de I. W. Volckmann. En las reflexiones destaca la diferencia entre la unidad objetiva de la conciencia y la unidad subjetiva, una diferencia paralela a la distinción entre juicios de experiencia y juicios de percepción. La clara diferencia de estas dos unidades es uno de los rasgos que distinguen a la segunda edición de la versión previa. En el fragmento de las lecciones destaca cómo Kant retoma la caracterización de las categorías que había desarrollado en 1772, como aquellos conceptos que nos permiten referir intuiciones a objetos al fijar posiciones en los distintos tipos de juicios, caracterización que coloca al inicio de la parte que modificó de la deducción trascendental para la segunda edición de la Crítica de la razón pura.

Editar y traducir los textos mencionados anteriormente, así como reunirlos en un solo volumen, representa una contribución muy valiosa para el estudio de la filosofía teórica de Kant. No solo es un lujo para los adeptos a este proyecto, sino sobre todo una gran ayuda para comprender con más facilidad y precisión la manera en que se fue gestando y transformando la Deducción trascendental, esa pieza clave de una de las principales obras de la filosofía occidental.

\section{Bibliografia}

CAIMI, Mario: Leçons sur Kant. La déduction transcendantale dans la deuxième édition de la Critique de la raison pure, París, La Sorbonne, 2007.

CARL, Wolfgang: Der schweigende Kant, Gotinga, Vandenhoek \& Ruprecht, 1989.

KANT, Immanuel: Gesammelte Schriften, Berlín, Akademie der Wissenschaften, Walter de Gruyter (AA), 1902ss.

KEMP SMITH, Norman: A Commentary to Kant's Critique of Pure Reason, Londres, Macmillan, 1923.

MOLEDO, Fernando: Los años silenciosos de Kant, Buenos Aires, Prometeo Libros, 2014.

STEPANENKO, Pedro: Categorias y autoconciencia en Kant: Antecedentes y objetivos de la deducción trascendental de las categorías, Ciudad de México, Universidad Nacional Autónoma de México, 2000.

TORRETTI, Roberto: Kant: Estudio sobre los fundamentos de la filosofía crítica, Santiago de Chile, Universidad de Chile, 1967.

VAIHINGER, Hans: Die transcendentale Deduktion der Kategorien in der 1. Auflage der Kr.d.r.V., Halle, Philosophische Abhandlugen, 1902.

VILLACAÑAS, José Luis: La formación de la Critica de la razón pura, Valencia, Universidad de Valencia, 1980.

VLEESCHAUWER, Herman J. de: La déduction transcendantale dans l'œuvre de Kant, La Haya, Nijhoff, 1937. 


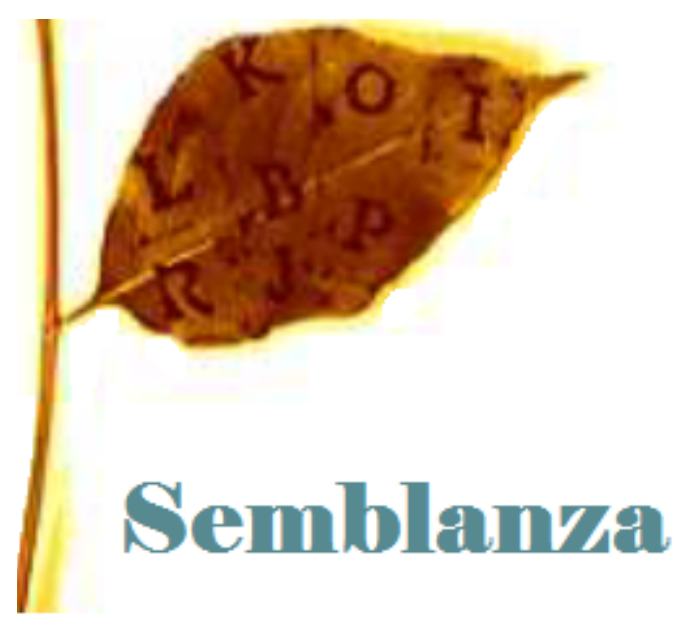




\title{
Juan Adolfo Bonaccini (16 de mayo de 1965 / 10 de julio de 2016)
}

\author{
DANIEL OMAR PEREZ ${ }^{1}$
}

Juan Adolfo Bonaccini nació en Argentina el 16 de mayo de 1965. Fue al colegio Nacional Mariano Moreno en Buenos Aires y realizó, posteriormente, su formación universitaria en la Universidad Federal de Río de Janeiro. Entre 1985 y 1989 cursó la graduación en filosofía y su trabajo de conclusión fue "Algunas consideraciones sobre el prefacio de la Fenomenología del Espíritu". Entre 1989 y 1993 realizó una maestría que llevó por título "La dialéctica en Kant y en Hegel”. Entre 1993 y 1997 desarrolló sus estudios de doctorado en Freiburg en 1996. Su tesis trató sobre "El concepto de la cosa en si en el idealismo alemán". Entre 2007 y 2009 estuvo East Leansing en la Michigan State University como profesor investigador en colaboración con Fredrich Rauscher para trabajar temas kantianos y hegelianos. Dio conferencias en varios países de Europa y América y también publicó en revistas y libros universitarios. Estudió varias lenguas, entre ellas latín y griego. Además del castellano y el portugués, hablaba inglés, francés, alemán e italiano. Leía la obra de Jorge Luis Borges, literatura en lengua alemana y en lengua portuguesa. Eso se notó siempre en la forma de escribir sus textos filosóficos.

La tarde del sábado 9 de julio de 2016 estuve en el bar Café com Arte, en Barão Geraldo (Campinas, São Paulo). Mientras conversaba con Francisco Coupelade y Carlos Andrés Duque, miré mi celular y vi varios mensajes de colegas y estudiantes diciendo que Tereza, su mujer, me estaba buscando. Ella solo tenía el número de mi teléfono de casa y mandaba a decir que Juan quería hablar conmigo. Volví rápido a casa. No sé por qué. Podría haber hablado desde donde estaba, pero mi primer impulso fue volver a casa como buscando refugio. Hablé con Tereza. Me dijo que estaban en el hospital y que era grave. Juan escuchaba lo que yo decía: "dejá de joder Juan, pasá la noche allí y mañana volvés a casa, tenemos que continuar trabajando en el artículo sobre ontología y semántica, no me vengas con b...", y también: "yo mañana te llamo al medio día para ver cómo fue todo, no me jodas". Ya habíamos hablado antes así y él me había hecho caso. El domingo 10 de julio, cuando iba caminando hacia donde está el teléfono de casa escucho que llama. Atiendo y Tereza dice: Juan murió.

Desde la década de 1990, los trabajos de Juan Bonaccini ya daban la pauta de lo que sería su tarea en filosofía hasta el año de su partida. Si bien abordó cuestiones teoréticas y prácticas, el fondo del problema que siempre le interesó estuvo afincado en la metafísica, su sistema, su sistematicidad, la reflexión sobre la existencia y los objetos a partir de las obras de Kant y Hegel. El a priori del espacio y del tiempo, el concepto de fenómeno, el estatuto de la cosa en sí desde la polémica de Kant y Jacobi, hasta su teoría de los diferentes conceptos de objetos en la primera Crítica, fueron exhaustivamente examinados y revistos a la luz de los nuevos resultados de sus investigaciones y debates. Sobre ese fundamento podemos ver aparecer sus trabajos de filosofía práctica acerca de la moral kantiana, la antropología y la teoría de los milagros. Los problemas de la censura moral, las paradojas éticas, la distinción entre ética y moral, la libertad y la naturaleza se encuentran en artículos y capítulos de libros. Durante toda su trayectoria hay también varios ensayos en torno de temas como la relación con la literatura, el lenguaje y el tiempo en Nietzsche, Descartes, San Agustin y Borges. Temas de su vida y de su investigación lo demoraron en la escritura de algunos libros y varios artículos. Todos los trabajos publicados fueron previamente presentados en eventos donde discutía con pasión y después reformulaba pacientemente sus argumentos e inclusive sus puntos de partida.

En 1995 y mientras hacía su doctorado, Juan Bonaccini fue hasta la reunión de la SBPC (Sociedade Brasileira para o Progresso da Ciência) en São Luiz de Maranhão, para presentar una

${ }^{1}$ UNICAMP. 
ponencia sobre parte de su trabajo de entonces. Lo vi de cabellos largos y de una barba inmensa, con una amabilidad y una curiosidad por la filosofía que no es común encontrar en medios académicos tomados por la burocratización del trabajo intelectual. En otros eventos donde estuve con él, no recuerdo que no haya disputado y rivalizado hasta pasar la línea de las reglas del ceremonial y la etiqueta. Así era en los encuentros de la Sociedade Kant Brasileira, de los coloquios Kant de Campinas en la UNICAMP o en las reuniones de la ANPOF. Solamente en los últimos años cambió el espíritu belicoso por una generosidad y cuidado con las palabras en las críticas que realmente emocionaba. Ya no tenía barba ni cabello. Parecía estar entregando todo lo que tenía en materia de conocimiento y fuerza física. Continuaba caminando de la misma forma, eso no lo cambió. Tampoco su pasión por la filosofía y su compromiso absoluto con cada problema que enfrentaba.

Kant y el problema de la cosa en sí en el idealismo alemán. Su actualidad y relevancia para la comprensión del problema de la filosofía es es el título del libro de Bonaccini, publicado en 2003 por la editora Relume-Dumará, de Rio de Janeiro y dedicado a su abuela, Manuela Rosa Aldasoro. Su contribución no es un trabajo 'histórico' de la filosofía, donde solamente se presenta el contexto de la discusión en el idealismo alemán, las posiciones de Kant, Reinhold y Jacobi, las tesis de Maimon, Schulze y Beck y los post-kantianos Fichte, Schelling y Hegel, bien como los Kantian scholars (como le gustaba decir a Juan) Prauss, Buchdahl y Allison. Lo que está en juego en el libro es, de inicio a fin, a partir de la tradición filosófica, la posibilidad de pensar un fundamento ontológico en la filosofía, o tal vez su imposibilidad:

La pregunta que nos hicimos a la luz de esa tradición fue la siguiente: que es lo que define a la filosofía si su historia pone en evidencia una pluralidad de sistemas, pensamientos y opiniones contrarios entre sí, una dialéctica sin coherencia aparente ni ostensivo progreso? La respuesta que intentamos alcanzar a partir de un análisis y de una confrontación entre las concepciones de Kant y de Hegel, antes de ofrecer una solución para el problema propuesto, sirvió para delimitar una aporía característica del pensamiento (del logos) en general y de la filosofía en particular. Su conclusión fue de un cierto modo escéptica. Conseguimos mostrar, con una estrategia inspirada en Kant y en Sexto, algo que no está lejos de aquello que Sócrates hacía con sus interlocutores en los primeros diálogos platónicos: reconocer la aporía y, a través de ella, admitir la parcialidad peculiar de cada abordaje de respuesta al problema en cuestión (Bonaccini 2003, 17).

El libro es al mismo tiempo historia de la filosofía y problematización filosófica contemporánea. En él están los problemas que insistieron en los textos de Juan Bonaccini: el fundamento ontológico y la sistematización del pensar.

Juan demoró bastante la publicación de los resultados de su investigación que acabaron siendo conocidos como Kant y el problema de la cosa en si en el idealismo alemán. El tiempo de reflexión valió la pena pues en más de una ocasión y sentados en algún bar de algún hotel en algún evento en Brasil, algún colega se aproximaba y comentaba cómo el libro ayudaba a entender el problema central del final del kantismo y el inicio del idealismo alemán. Su lectura se tornó necesaria en Brasil y varias cátedras de filosofía moderna incluían su título en la bibliografía.

Durante algunos años discutimos el lugar y el método de la antropología en Kant y lo que significaría la temática de la aplicación de las reglas morales en el proyecto filosófico kantiano. Bonaccini no disociaba ni colocaba en un lugar menor las cuestiones de la aplicabilidad del imperativo categórico. Por eso la importancia de la antropologia. En lo que se refiere al método de la antropología, su idea de 'analogía' fue desarrollada en uno de sus artículos, después de varios encuentros y que lleva por título "Antropología, ciencia de la naturaleza humana 'por analogia"”. Tal vez este fragmento sea útil para entender desde donde Bonaccini articulaba sus reflexiones de filosofía práctica:

Los conceptos de antropología pragmática y de hombre como ciudadano delmundo permiten formular un concepto de naturaleza humana no necesariamente metafísico, ya que son formulados en un contexto que no es a priori. Por otro lado, la idea de naturaleza común de la especie, es útil a la educación, el arte, la historia, la política e la moral pues puede ser pragmáticamente utilizada, y su resultado puede ser proyectado y hasta corroborado a luz de la observación, de la experiencia y de la analogía como un principio de convivencia y perfeccionamiento social. Si bien el concepto ya está comprometido con el proyecto kantiano de fomentar el progreso moral da humanidad, justamente por parte de la Antropología pragmática que puede ser considerada moral, el peso metafísico puede ser resuelto en términos normativos. Lo empírico se subordina a una finalidad moral que se realiza a través de la acción política en el campo de la historia de todos los hombres. No se ve ningún prejuicio para la 
humanidad o para la filosofía con una opción semejante. Antes podemos entenderlo una herramienta para pensar mejor y conducir nuestras vidas de acurdo con nuestra naturaleza (Bonaccini, 2010).

De este modo, se ve claramente la necesidad de pensar la razón práctica como realizada en la naturaleza. Eso era también parte de la filosofía, al menos como lo entendía Bonaccini. La moral kantiana no era para el filósofo algo ajeno a su reflexión acerca de la vida personal. Sus conflictos existenciales tenían el trazo de lo que se discutía en los encuentros kantianos, dentro y fuera de la sala de conferencias. Tal vez por eso él entendía que no era suficiente formular el imperativo categórico: un filósofo debería mostrar también su aplicabilidad.

En su último proyecto de investigación que tituló "Ontología, epistemología y semántica: sobre la teoría kantiana acerca de la estructura objetual del mundo", retomaba los inicios de sus estudios sobre fenómeno y cosa en sí, solo que de un modo diferente. Las polémicas en Campinas (UNICAMP) sobre Ontología y Semántica le habían hecho ampliar su perspectiva y ya comenzábamos a tener coincidencias cada vez más fuertes. La insistente cuestión con la ontología que habría hecho Kant en la primera parte de la Crítica de la razón pura lo llevaba a compartir las tesis de Luis Cesar Yanzer Portela. Entretanto, ambos acabaron coincidiendo en que también había cuestiones semánticas con relación a las categorías que deberían ser tenidas en consideración. Si hoy pudiésemos continuar escribiendo nuestro trabajo interrumpido, aquel artículo final le diría que Kant tal vez esté proponiendo una cuestión de henología, una cuestión acerca de la posibilidad de la unidad, de 'lo diverso de la intuición' en 'algo' que se presupone dado, desde allí deberíamos poder pensar la relación con las categorías y finalmente la cuestión de objeto. Como decían Kant y Lambert en sus famosas correspondencias: no se puede comenzar por el ente porque es ya una síntesis, debemos comenzar por algo aún más simple.

En 2015 Juan participó del Coloquio Kant de Campinas (UNICAMP). Se quedó en casa, se levantaba temprano y participaba desde el inicio hasta el final. Intervenía en cada comunicación. Cada aporte era una clase didáctica, rigurosa, precisa y gentil. En el tiempo libre estuvo trabajando con el profesor Portela, de la Unioeste, sobre el problema de la ontología y la semántica. "Me gustaría ver a mis hijos entrando en la universidad, pero no va a poder ser. Tengo poco tiempo", me dijo. 


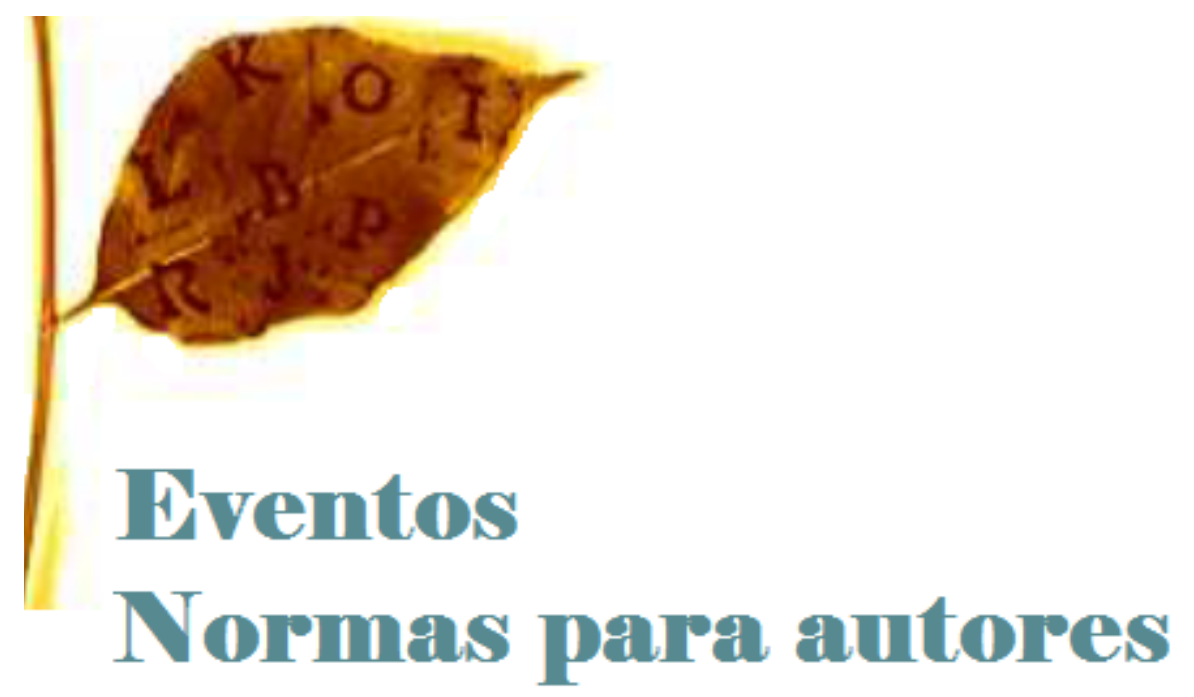




\section{CONVOCATORIA}

Atendiendo a su objetivo de "fomentar el estudio de la filosofía de Kant, de su época, de sus antecedentes y de su influjo posterior, así como de los temas relacionados con su pensamiento, su método filosófico y su posible desarrollo y apropiación actuales" (Estatutos, art. 2), la Revista de Estudios Kantianos (REK) desea impulsar especialmente los estudios sobre la recepción del pensamiento de Kant en el ámbito hispanohablante, enfatizando especialmente autores y obras pioneros que han proyectado líneas de reflexión y comprensión significativas de la filosofía de Kant, sea a partir de su magisterio, o bien sea mediante la publicación de traducciones y obras originales.

Es por ello que la $R E K$ desea convocar a todo(a)s los académico(a)s e investigadore(a)s en lengua española interesados a enviar sus trabajos sobre

\section{La recepción de Kant en México}

La fecha límite para la entrega de los trabajos es el 10 de marzo del 2018. Los trabajos estarán sujetos a las normas de edición de la $R E K$ (http://sekle.org/call-for-papers-revista.html). Los originales aprobados serán publicados en el No. 5 de la $R E K$ que aparecerá en 2018.

Atentamente

Dulce María Granja Castro

Gustavo Leyva Martínez 


\title{
IV Congreso internacional de la Sociedad de Estudios Kantianos en Lengua Española (SEKLE)
}

\section{"La actualidad de la Crítica de la razón pura"}

\author{
15-19 de octubre de 2018 \\ Universitat de València \\ Facultad de Filosofía y Ciencias de la Educación
}

Por la presente, se abre la convocatoria de presentación de originales para el IV Congreso internacional de la Sociedad de Estudios Kantianos en Lengua Española (SEKLE), que tendrá lugar en la Facultad de Filosofía y Ciencias de la Educación de la Universidad de Valencia del 15 al 19 de octubre de 2018. El motivo del congreso será "La actualidad de la Crítica de la razón pura". No obstante, las propuestas relacionadas con cualquier aspecto del pensamiento kantiano, así como con sus fuentes y su recepción, serán bienvenidas.

Entre los ponentes confirmados se hallan,

- $\quad$ Adela Cortina (Universidad de Valencia)

- Claudio La Rocca (Università di Pisa)

- Heiner F. Klemme (Martin-Luther-Universität Halle-Wittenberg)

- Onora O’Neill (University of Cambridge)

- Pablo Oyarzún (Universidad de Chile)

En las sesiones semiplenarias intervendrán numerosos ponentes de diferentes países. Entre ellos se encuentran, por orden alfabético, Alba Jiménez (Madrid), Ana Andaluz (Salamanca), Antonio López Molina (Madrid), Bernd Dörflinger (Tréveris), Berta Pérez (Valencia), Catalina González (Bogotá), Cinta Canterla (Sevilla), Claudia Jáuregui (Buenos Aires), Costantino Esposito (Bari), Édgar Maraguat (Valencia), Faustino Oncina (Valencia), Fernando Moledo (Buenos Aires), Gustavo Leyva (Ciudad de México), Hernán Pringe (Buenos Aires), Jesús Conill (Valencia), Juan Manuel Navarro (Madrid), Julio del Valle (Lima), Manuel Jiménez (Valencia), Margit Ruffing (Maguncia), Mario Caimi (Buenos Aires), Maximiliano Hernández (Salamanca), Pedro Ribas (Madrid), Pedro Stepanenko (Ciudad de México), Salvi Turró (Barcelona), Sergio Sevilla (Valencia) y Vicente Durán (Bogotá). 
Los autores doctores deben enviar el título y el resumen de su ponencia (hasta un máximo de 100 palabras), justo con sus datos institucionales, hasta el 15 de enero de 2018 a la dirección de correo electrónico sekle.kant4@gmail.com. Los autores no doctores deben remitir, además de lo anterior, el texto completo de su ponencia. Los textos deben estar redactados preferentemente en castellano, pero se admitirán igualmente originales en alemán o inglés. Los autores serán comunicados acerca de la aceptación de sus propuestas antes del 31 de marzo de 2018.

Para más información: http://kant4.blogs.uv.es/convocatoria

\section{English version}

The call for papers is hereby opened for the $4^{\text {th }}$ International Congress of the Society for Kantian Studies in the Spanish Language (SEKLE), that will be held from 15-19 October 2018 in the Faculty of Philosophy and Education Sciences at University of Valencia. The leitmotiv of the conference will be "The current relevance of the Critique of Pure Reason". Nevertheless, all contributions on any aspect of Kantian thought are welcome, as well as on its sources and reception.

Among the confirmed keynote speakers are,

- $\quad$ Adela Cortina (Universidad de Valencia)

- Claudio La Rocca (Università di Pisa)

- Heiner F. Klemme (Martin-Luther-Universität Halle-Wittenberg)

- Onora O’Neill (University of Cambridge)

- $\quad$ Pablo Oyarzún (Universidad de Chile)

The main lectures will be held by many speakers from different countries, among them - in alphabetical order - Alba Jiménez (Madrid), Ana Andaluz (Salamanca), Antonio López Molina (Madrid), Bernd Dörflinger (Trier), Berta Pérez (València), Catalina González (Bogotá), Cinta Canterla (Sevilla), Claudia Jáuregui (Buenos Aires), Costantino Esposito (Bari), Édgar Maraguat (València), Faustino Oncina (València), Fernando Moledo (Buenos Aires), Gustavo Leyva (Mexico City), Hernán Pringe (Buenos Aires), Jesús Conill (València), Juan Manuel Navarro (Madrid), Julio del Valle (Lima), Manuel Jiménez (València), Margit Ruffing (Mainz), Mario Caimi (Buenos Aires), Maximiliano Hernández (Salamanca), Pedro Ribas (Madrid), Pedro Stepanenko (Mexico City), Salvi Turró (Barcelona), Sergio Sevilla (València) and Vicente Durán (Bogotá).

PhDs will submit title and abstract (100 words maximum), together with academic affiliation, until 15 January 2018 to the email address sekle.kant4@gmail.com. Non-PhDs will also submit the whole text of the paper. Proposals will be written in Spanish, German or English. Notice stating the acceptance of proposals will be given before 31 March 2018.

More information: http://kant4.blogs.uv.es/convocatoria 


\section{Comité Organizador / Organizing Committee}

Pedro Jesús Teruel (Universitat de València), Coordinación, pedro.teruel@uv.es

Fernando Moledo (Universidad de Buenos Aires), Secretaría del congreso, fernandomoledo@filo.uba.ar Alba Jiménez (Universidad Autónoma de Madrid), Plan de comunicación, alba.jimenez@,uam.es Jesús Conill (Universitat de València), Programa cultural, jesus.conill@uv.es

\section{Comité Científico / Scientific Committee}

Claudia Jáuregui (Universidad de Buenos Aires), Gustavo Leyva (Universidad Autónoma Metropolitana, Ciudad de México), Jacinto Rivera de Rosales (Universidad Nacional de Educación a Distancia), Jesús Conill (Universitat de València), Julio del Valle (Pontificia Universidad Católica de Perú), María Xesús Vázquez Lobeiras (Universidade de Santiago de Compostela), Pablo Oyarzún (Universidad de Chile), Pedro Stepanenko (Universidad Nacional Autónoma de México), Rogelio Rovira (Universidad Complutense de Madrid), Vicente Durán (Universidad Javeriana, Bogotá), Wilson Herrera (Universidad del Rosario, Bogotá).
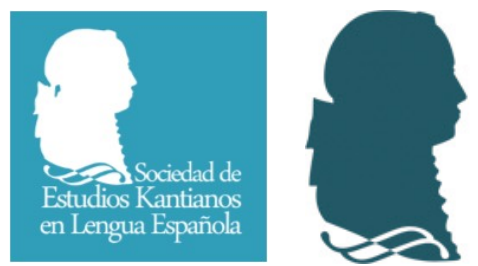

Revista

de 


\title{
CONVOCATORIA
}

\section{Multilateral Kant Colloquium}

With the support of SISK - Società Italiana di Studi Kantiani

\section{"Kant and the Contemporary World: Philosophy, Science, Politics"}

October 11-13, 2018

\author{
Department of Political and Social Sciences, University of Catania \\ Via Vittorio Emanuele II, 49
}

The $8^{\text {th }}$ Edition of the Multilateral Kant Colloquium will be held at the Department of Political and Social Sciences of the University of Catania, October 11-13, 2018. Scholars from all over the world will be involved in presentations and discussions of the most up-to-date research on Kant's philosophy.

With the support of the Società Italiana di Studi Kantiani, the conference is part of an annual initiative started in 2008 by an international group of scholars keen on promoting informed debate on the latest developments of Kant scholarships. Previous editions took place in Italy ("Kant Today", Verona and Padua), Portugal ("What is Man? - Was ist der Mensch?”, Lisbon 2010), Germany ("Kant and Antinomical Thinking", Mainz 2011; and "Kant and His Critics", Halle 2017), Brazil ("Kant and the Metaphors of Reason", Tiradentes 2013), Spain ("Kant's Short Writings Kleine Schriften", Madrid 2014), and the United States ("Kant on Violence, Revolution, and Progress, Hempstead, NY, 2016).

The current edition aims at highlighting the relevance of Kantian thought with respect to the contemporary world, which becomes increasingly acknowledged in a wide number of issues crosscutting both the theoretical and practical sides of his work.

The Colloquium is addressed to scholars at an advanced stage of their career as well as junior researchers with an interest in Kantian philosophy. Participants will find the ideal context for high quality feedback on their ongoing work and challenging discussions. Over three days, the conference will be structured around 10 invited talks and presentations selected through a call for papers. 
Paper abstracts are welcome for the 8th Edition of the Multilateral Kant Colloquium "Kant and the Contemporary World: Philosophy, Science, Politics". The theme is to be intended in the broadest possible sense, as involving any aspect of Kant's philosophy with an impact on issues debated in contemporary philosophy and science, as well as relevant for the most pressing political problems of our time.

The official language is English, but participants can submit and present their papers in Italian, German, Portuguese, Spanish or French. If papers are not written in English, an English version of the texts should be made available at the moment of the presentation. An international committee chaired by Prof. Luigi Caranti will select the papers.

Abstracts from scholars at an advanced stage of their career as well as junior researchers should be prepared for blind review and be confined between 600 and 800 words. Contact information should be sent in a separate file. Deadline for submission is February $1^{\text {st }} \mathbf{2 0 1 8}$. Please send abstracts to: denise.celentano@ehess.fr. Notice of acceptance will be issued by March 1st 2018. Each presentation will last 30 minutes, followed by 15 minutes for questions and debate.

\section{Accommodation}

Rooms at Camplus d'Aragona (few minute walk from the Department) are available at the following rates (breakfast included): $50 €$ single, $55 €$ double as single, $60 €$ double, $75 €$ triple. Reservations should be made by April $30^{\text {th }} 2018$, specifying your participation to the Multilateral along with the rates mentioned. Alternatively, there are many good and cheap accommodations in Catania that you may book on your own.

\section{More information}

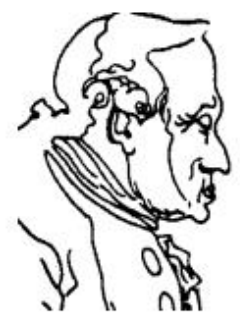

\section{Società Italiana di Studi Kantiani}

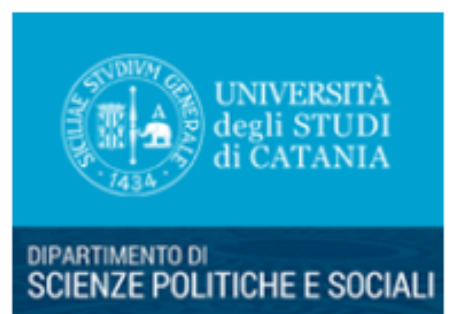




\section{Normas para autores/as}

La revista publica trabajos redactados en lengua castellana, alemana o inglesa y referidos a cualquiera de los ámbitos relativos a la filosofía kantiana o en los que la presencia de ésta tenga una relevancia significativa. Las contribuciones deben ser originales y su extensión no podrá superar los 80000 caracteres con espacios. Las reseñas no excederán los 16000 caracteres con espacios.

\section{Encabezamiento}

El texto será precedido por título, resumen (máx. 100 palabras) y hasta cinco palabras clave, tanto en la lengua propia del artículo como en inglés (si la lengua empleada en el trabajo es el castellano) o en castellano (si el trabajo está redactado en inglés o alemán).

\section{Estilo}

\subsection{Requisitos formales}

Los textos serán redactados en Source Sans Pro (o en su defecto en Times New Roman), 9 puntos (o 12 respectivamente), interlineado múltiple 1,15 .

Las referencias relativas a una cita textual aparecerán preferiblemente en cuerpo de texto, indicando entre paréntesis autor y año seguido de dos puntos y número de página, según el modelo: “...tal y como indica el naturalista inglés (Darwin 1871: 32)...”. Ello no excluye que se abra una reflexión complementaria, si así se estima oportuno, en nota a pie de página, a 7 puntos (10 para Times). Cuando se indique que parte de la cita ha sido omitida se emplearán tres puntos entre corchetes [...]. Si la cita es en lengua distinta a la del artículo, se tratará de buscar su traducción en alguna edición en castellano y se añadirá entre corchetes la cita en la lengua original (en caso de que no se disponga de una traducción, el autor indicará que la misma ha sido elaborada por él mismo mediante la abreviatura a. trad., o bien en una nota a pie de página si todas las traducciones son propias); por ejemplo “...tal y como indica el naturalista inglés...” [“...as the English naturalist states...”]. Cuando las citas textuales superen los 4 renglones se colocarán aparte, a 8 puntos (11 para Times), en un párrafo justificado y sangrado en primera lína a 1,25 puntos.

Los encabezados de los apartados y subapartados se indicarán mediante la siguiente numeración: 1, 1.1, 1.1.1... etc., sin cursiva ni negrita. La enumeración de listados empleará los indicativos i), ii), iii) etc.

La cursiva se reserva para los títulos de obras y palabras extranjeras. Para resaltar el énfasis puesto en una palabra se utilizarán comillas simples '...'.

Los números indicativos de las notas a pie de página (que en ningún caso se colocarán al final del documento, sino al final de cada página) deben encontrarse antes de los signos de puntuación.

El primer párrafo de cada apartado no estará sangrado, mientras que los restantes sí lo estarán en primera línea a 1,25 puntos. Entre párrafos se dejará un renglón en blanco.

Es aconsejable que los autores revisen los artículos publicados previamente para una mejor adaptación a las normas de estilo. 


\subsection{Citaciones kantianas}

En el caso de obras de Kant, en cuerpo de texto y nota se aludirá a ellas empleando las abreviaturas establecidas por la Kant-Forschungsstelle de Maguncia. Las abreviaturas se hallan publicadas en su página web (http://www.kant.uni-mainz.de/), pestaña 'Kant-Studien', sección 'Hinweise für Autoren'.

\section{Referencias bibliográficas}

Las referencias bibliográficas completas aparecerán al final de la contribución, en un apartado ad hoc bajo el título "Bibliografía", por orden alfabético aplicado a los apellidos de los autores. En el caso de que varias publicaciones del mismo autor coincidan en el mismo año, se las distinguirá con letras en subíndice (1999a, 1999b).

3.1. Citas de libros

Procederán según el siguiente modelo:

MORENO, Juan: Los retos actuales del darwinismo. ¿Una teoría en crisis?, Madrid, Síntesis, 2008.

En el caso de que en el cuerpo de texto se citen textos procedentes de traducciones, en la bibliografía se especificará como sigue:

DARWIN, Charles R.: The Descent of Man and Selection in Relation to Sex, Londres, John Murray, 1871, 1882 ( $2^{\mathrm{a}}$ ed.). Los textos citados provienen de la traducción castellana realizada por Julián Aguirre: El origen del hombre y la selección en relación al sexo, Madrid, EDAF, 2006 ( $2^{\mathrm{a}} \mathrm{ed}$.).

3.2. Capítulos de libro

KINSBOURNE, Marcel: "Integrated field theory of consciousness", en MARCEL, A. y BISIACH, E. (eds.): Consciousness in Contemporary Science, Oxford, Oxford University Press, 1988.

\section{3. Artículos}

TINBERGEN, Nikolaas: "On aims and methods of Ethology", Zeitschrift für Tierpsychologie 20 (1963) 410-433.

\section{Revisión}

Cada autor/a revisará detenidamente que de su envío haya sido eliminado cualquier indicio que pudiera denotar su identidad. En el caso de que se aluda a publicaciones propias, se sustituirá todos los datos por la indicación "[Datos eliminados para favorecer la anonimidad del envío]". Del mismo modo, se cuidará de que el documento no contenga información sobre la autoría en sus propiedades informáticas.

\section{Envío}

Los trabajos deberán ser enviados a través de la plataforma virtual:

https://ojs.uv.es/index.php/REK/ 


\section{Instructions for contributors}

The journal publishes papers in Spanish, German or English dealing with Kantian philosophy or with issues raised in it. Contributions must be original and should not exceed 25 pages (90000 characters with spaces). Reviews should not exceed 5 pages (18000 characters with spaces).

\section{Heading}

The text will be headed by title, abstract (max. 100 words) and key words (max. 5) in both the language in which the work is written and English (if written in Spanish) or Spanish (if written in English or German).

\section{Style}

\subsection{Formal items}

The text will be written in Source Sans Pro (or Times New Roman), 9 points (or 12 p. respectively), multiple spacing 1,15 . The textual quotes will appear in the text self, by referring to author and year followed by colon and page number. For example: “...so as pointed out by the British naturalist (Darwin 1871: 32)..." If considered suitable, a further discussion in a footnote is not excluded (7 points or 10 for Times). To indicate that a part of the quoted text has been omitted three points will be used in brackets (...). If the quote is in a different language, the author should look for a translation, adding the original quote in brackets as follows: “...tal y como indica el naturalista inglés...” [“...as the English naturalist states..."]. If no translation is available, the author will indicate that it has been drawn up by himself by the abbreviation a. trans. or in a footnote if all translations have been made by the author. When quotations exceed 4 lines will be placed apart, 8 points (11 for Times), in a justified paragraph and bleeding in top line to 1,25 points.

The headings of the sections and subsections shall be indicated by the following numbers: $1,1.1$, 1.1.1., etc., without italics nor bold. To enumerate the elements of a list: i), ii), iii), etc.

Reserves italics for titles of works and foreign words. To highlight the emphasis on a word will be used single quotes '...'

The indicative numbers for footnotes (which in any case will be placed at the end of the document, but at the end of each page) must meet before punctuation.

The first paragraph of each section will not be bleeding, while the remaining will be bleeding in first line to 1,25 points. White line between paragraphs are reserved.

It is advisable that the authors review the previously published articles to better adaptation to the style rules.

\subsection{Kantian quotes}

The citation of Kantian texts will employ the abbreviations established by the Kant-Forschungsstelle Mainz (see website http://www.kant.uni-mainz.de/, 'Kant-Studien', section 'Hinweise für Autoren'). 


\section{Bibliography}

The complete references will be indicated (in alphabetic order of the name of the authors) at the end of the contribution, in a section entitled 'Bibliography'. Should different publications of an author be edited in the same year, they will appear with subscript characters (1999a, 1999b).

\subsection{Books}

The following model will be employed:

Moreno, Juan: Los retos actuales del darwinismo. ¿Una teoría en crisis?, Madrid, Síntesis, 2008.

Translated texts will be indicated as follows:

Darwin, Charles R.: The Descent of Man and Selection in Relation to Sex, London, John Murray, 1871, 1882 (2nd ed.). Spanish translation by Julián Aguirre: El origen del hombre y la selección en relación al sexo, Madrid, EDAF, 2006 (2nd ed.).

\subsection{Book chapters}

Kinsbourne, Marcel: "Integrated field theory of consciousness", en MARCEL, A. y BISIACH, E. (eds.): Consciousness in Contemporary Science, Oxford, Oxford University Press, 1988.

\subsection{Papers}

Tinbergen, Nikolaas: “On aims and methods of Ethology”, Zeitschrift für Tierpsychologie 20 (1963) 410-433.

\section{Revision}

Each author will make sure that all indication of identity has been removed. In case of quotation of the author's own publications, the reference will be replaced by the sentence "[Reference removed to guarantee anonymous review]". The author will take care that the file does not include personal information within its digital properties.

\section{Sending}

Contibutions should be uploaded to:

https://ojs.uv.es/index.php/REK/ 